\title{
MicroRNA-212/132 Family is Involved in the Regulation of Long-Term Spatial Memory and Synaptic Remodeling
}

\author{
Dissertation \\ for the award of the degree \\ "Doctor of Philosophy" (Ph.D.) \\ of the Georg-August-Universit t, Göttingen
}

within the doctoral program

"Genes and Development"

of the Göttingen Graduate School for Neuroscience and Molecular Biosciences (GGNB) of the Georg-August University School of Science (GAUSS)

submitted by

Erdem Erikçi

from Ankara/Turkey

Göttingen, 2013 


\section{$\underline{\text { Thesis Committee }}$}

Dr. Kamal Chowdhury $1^{\text {st }}$ Referee

Prof. Dr. Ernst Wimmer

$2^{\text {nd }}$ Referee

Prof. Dr. Michael Kessel
Department of Molecular Cell Biology, Max Planck Institute for Biophysical Chemistry, Göttingen

Department of Developmental Biology, Georg-August-University Göttingen

Department of Developmental Biology, Max Planck Institute for Biophysical Chemistry, Göttingen

\section{Extended Thesis Committee}

Prof. Dr. Dr. Hannelore Ehrenreich

Prof. Dr. Gregor Eichele

Dr. Jeong Seop Rhee
Clinical Neurosciences, Max Planck Institute for Experimental Medicine

Department of Genes and Behavior, Max Planck Institute for Biophysical Chemistry

Neurophysiology Group, Max Planck Institute for Experimental Medicine

Date of oral exam: 


\section{$\underline{\text { Affidavit }}$}

I hereby declare that my PhD thesis entitled "MicroRNA-212/132 family is involved in the regulation of long-term spatial memory and synaptic remodeling" has been written independently with no other sources and aids than quoted.

Erdem Erikçi, Göttingen, December 2013

This work has been generated at the Max Planck Institute for Biophysical Chemistry, Department of Molecular Cell Biology in Göttingen, in the research group of Dr. Kamal Chowdhury. 
Dedicated to my grandfather Şevki Erikçi.

(1924-2013)

I will miss you 


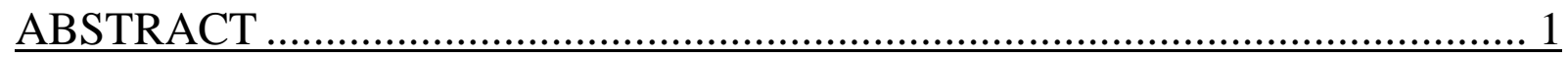

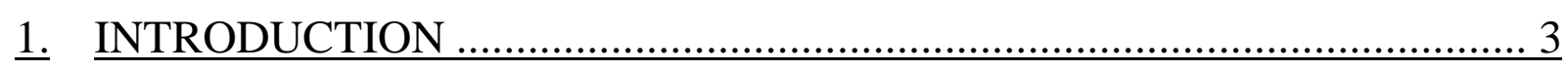

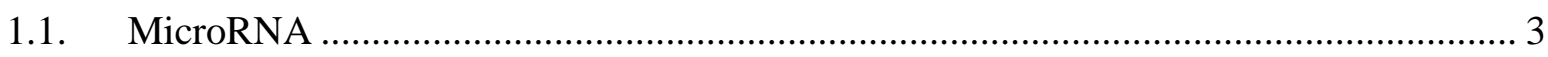

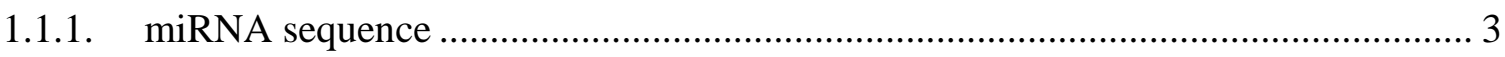

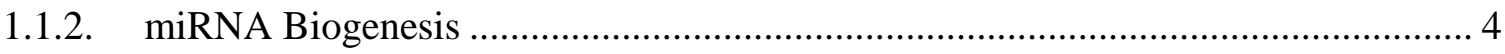

1.1.3. miRNA function ................................................................................... 7

1.1.4. Regulation of miRNA activities........................................................................ 8

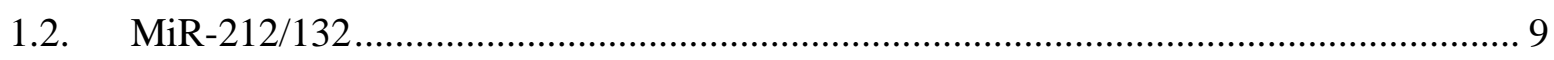

1.2.1. miR-212/132 gene family structure and expression ………................................. 9

1.2.2. miR-212/132 function ................................................................................... 13

1.2.2.1. miR-212/132 in neuronal morphogenesis.................................................... 14

1.2.2.2. miR-212/132 in newborn neuron integration .............................................. 16

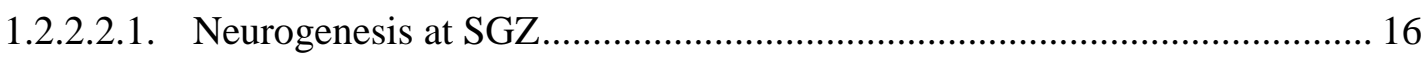

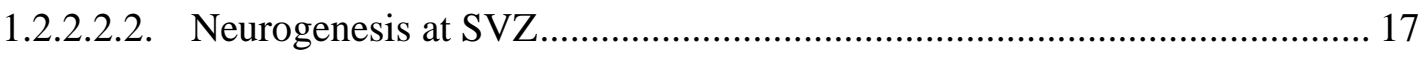

1.2.2.3. miR-212/132 in dopaminergic neuron differentiation ..................................... 17

1.2.2.4. miR-212/132 in synaptogenesis................................................................ 18

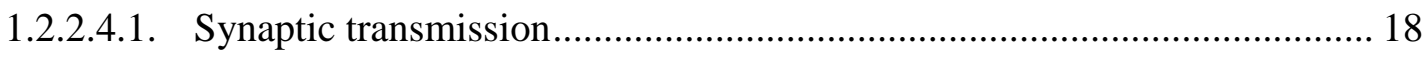

1.2.2.4.2. Ocular dominance plasticity ................................................................... 20

1.2.2.5. miR-212/132 in behavior ...................................................................... 21

1.2.2.5.1. miR-212/132 in learning and memory ................................................... 21

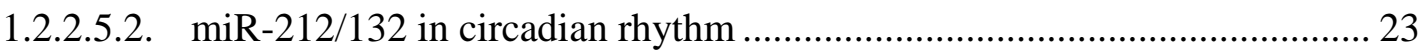

1.2.2.5.3. $\mathrm{miR}-212 / 132$ in drug addiction ............................................................... 25

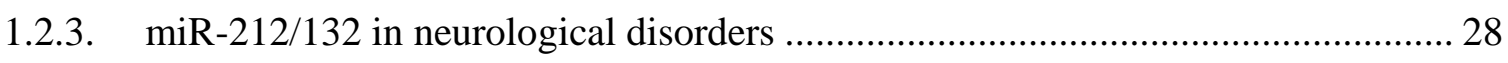

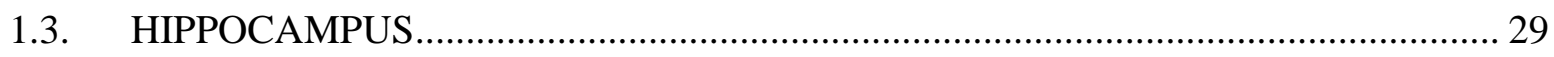

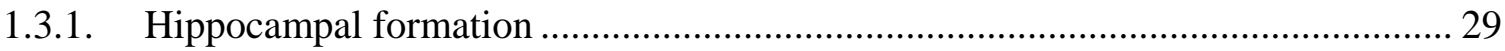

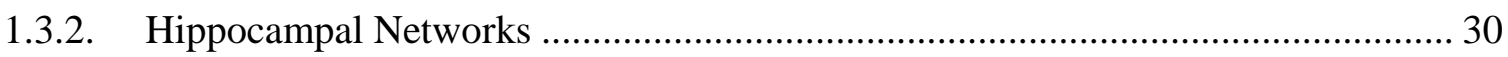




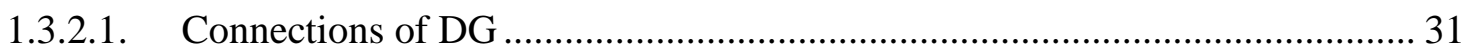

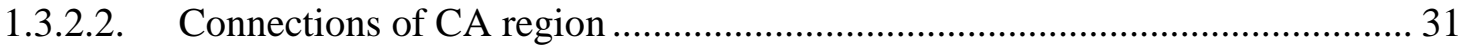

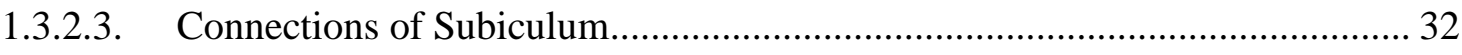

1.3.2.4. Connections of presubiculum and parasubiculum ......................................... 33

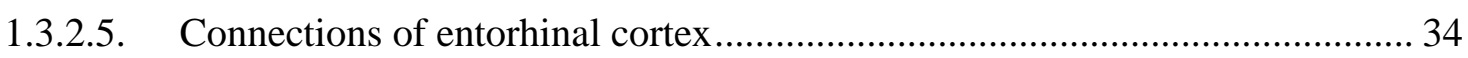

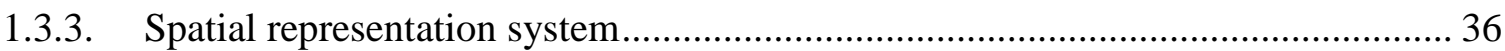

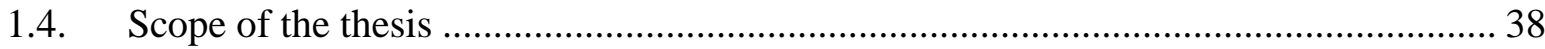

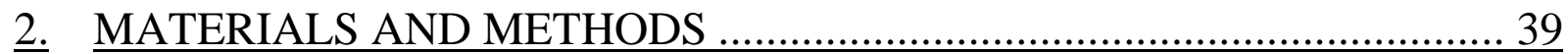

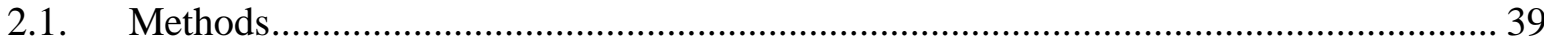

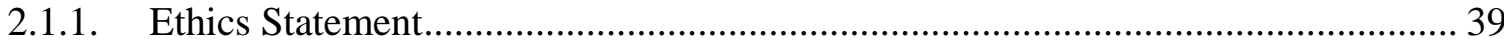

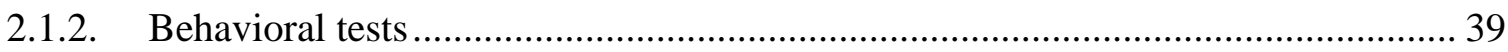

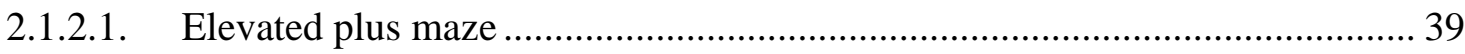

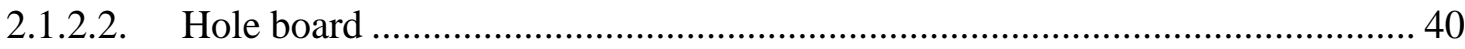

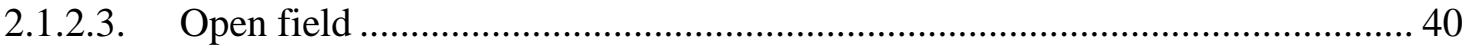

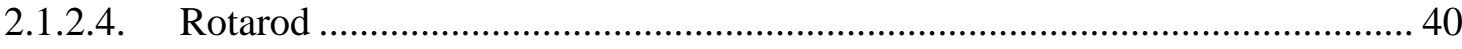

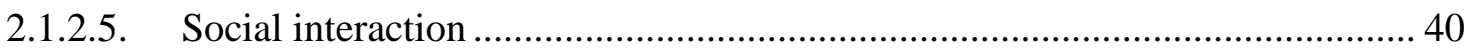

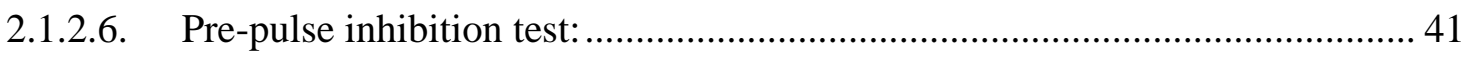

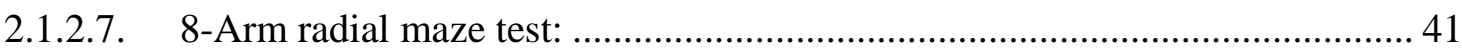

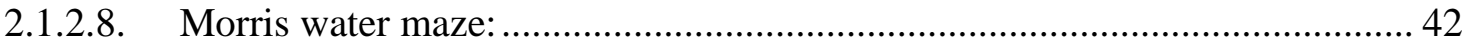

2.1.2.9. Cued and contextual fear conditioning: ........................................................ 42

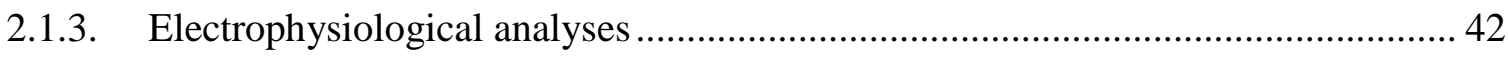

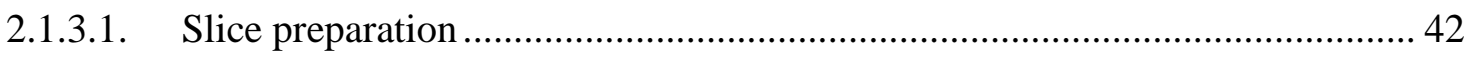

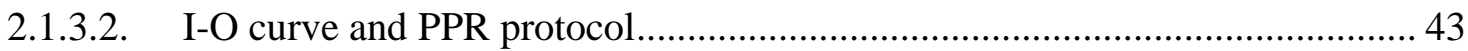

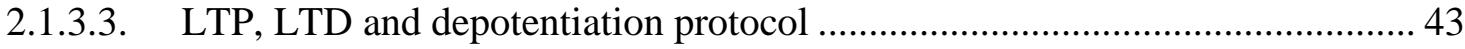

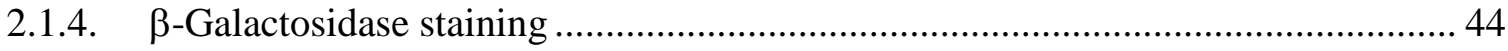




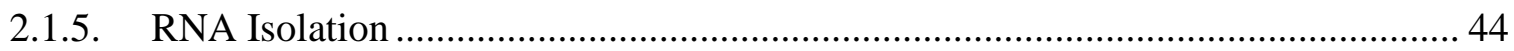

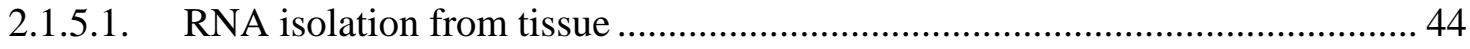

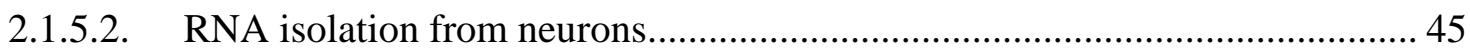

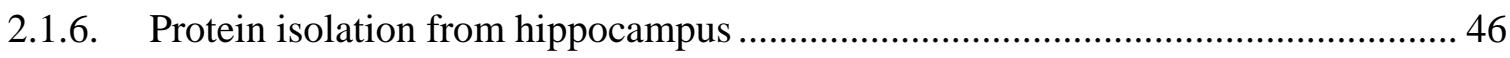

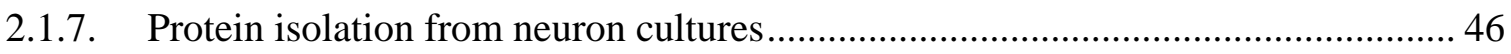

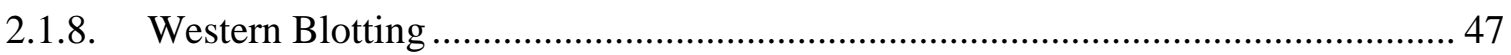

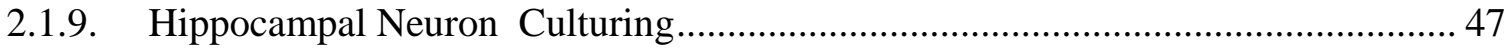

2.1.9.1. Poly-L-lysine coated plate preparation ......................................................... 47

2.1.9.2. Hippocampus dissection from embryonic brains .................................... 48

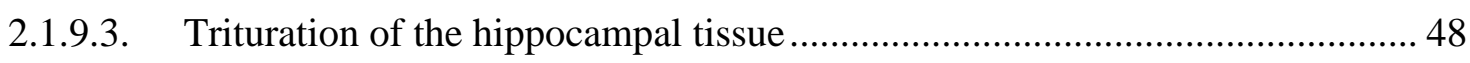

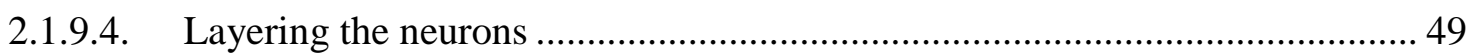

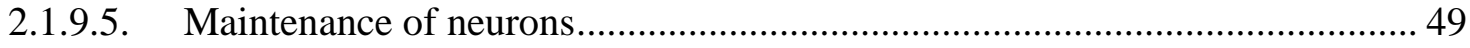

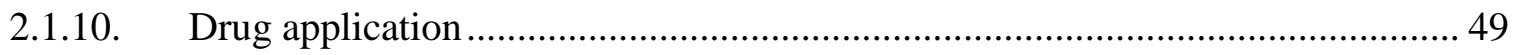

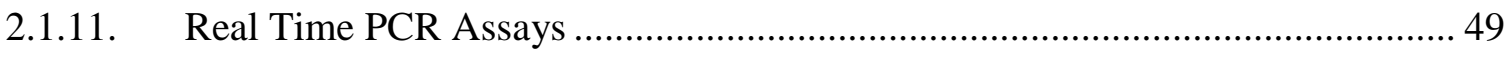

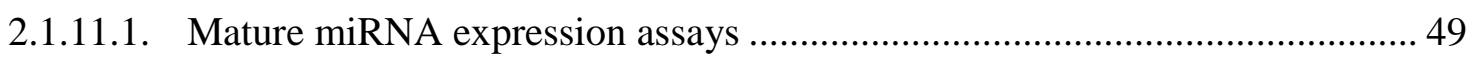

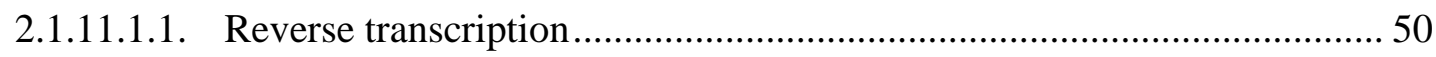

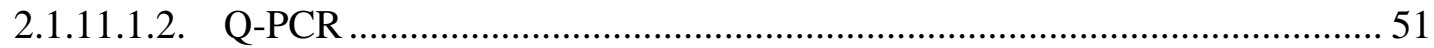

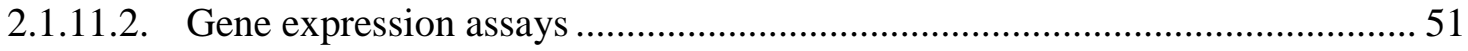

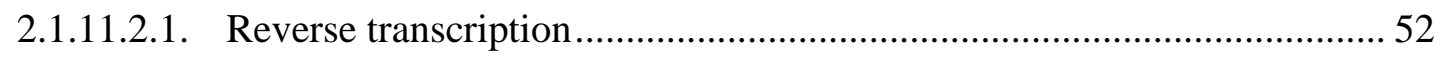

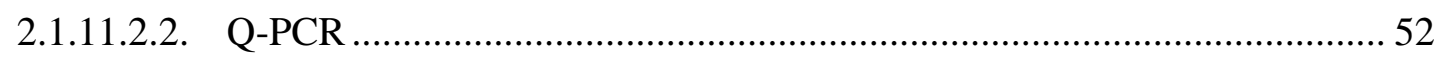

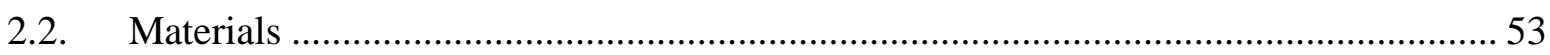

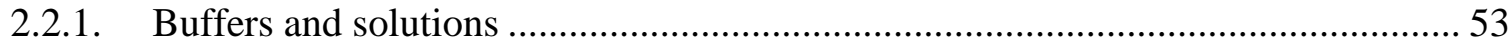

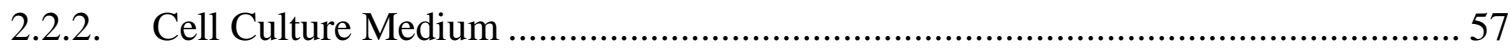

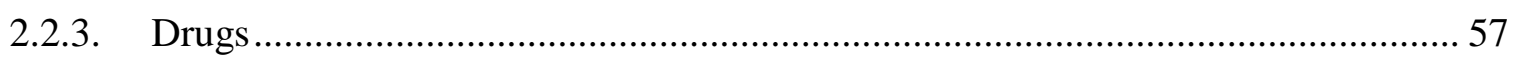

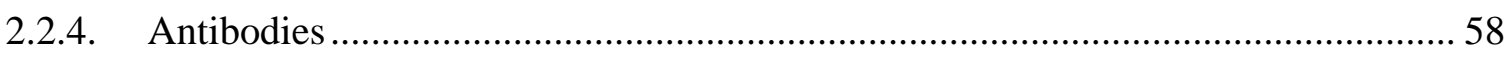


2.2.5. Gene and miRNA Expression Assays

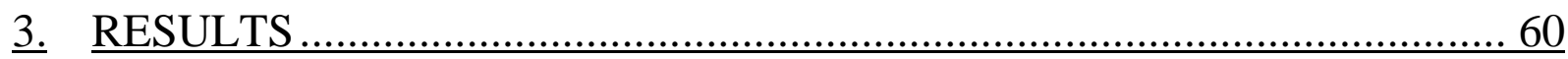

3.1. miR-212/132 mull mutant mice longevity and body weight ..................................... 60

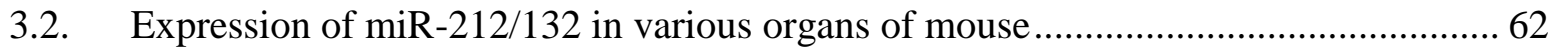

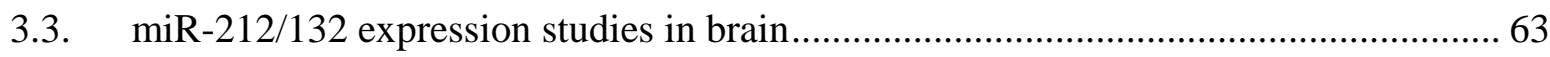

3.3.1. Arguments to utilize miR-212/132 $2^{-/-}$mice for $\beta$-galactosidase staining ................. 63

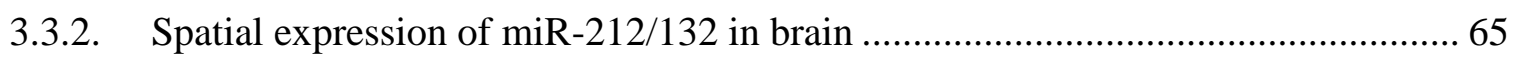

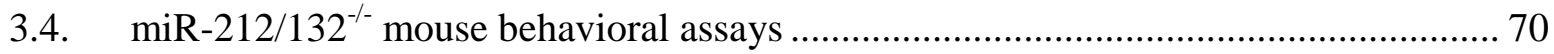

3.4.1. Anxiety levels, explorative activity, motor balance and coordination of miR-

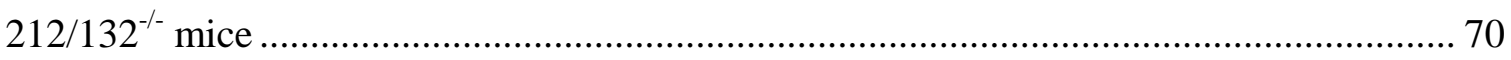

3.4.2. Sensorymotor gating, sociability, conditional learning and working memory of miR-212/132

3.4.3. Activity levels and spatial memory of miR-212/132 ${ }^{-/-}$mice................................. 75

3.5. Electrophysiological characterization of miR-212/132 ${ }^{-/-}$mice ..................................... 78

3.5.1. miR-212/132 increases the synaptic facilitation without affecting the synaptic

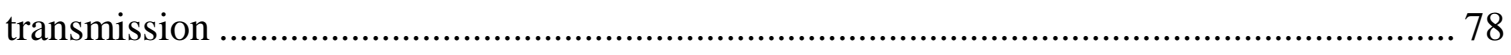

3.5.2. $\mathrm{miR}-212 / 132$ is essential for proper regulation of L-LTP but not for E-LTP ........ 79

3.5.3. miR-212/132 does not have significant influence on LTD and depotentiation ...... 80

3.6. The molecular functions of miR-212/132 in hippocampal neurons ........................... 82

3.6.1. Primary hippocampal neuron cultures as experimental setup.............................. 82

3.6.2. Induction of mature miR-212/132 production in hippocampal neuron cultures..... 84

3.6.3. Impaired immediate early gene regulation ..................................................... 85

3.6.4. Influence of miR-212/132 on SIRT1-miR-134-CREB pathway ............................ 90

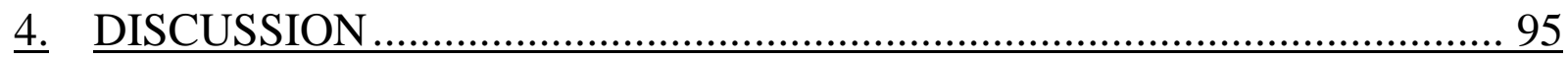

4.1. The correlation between miR-212/132 expression level and phenotype in various tissues 95 


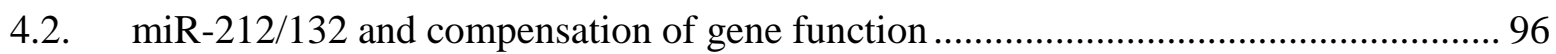

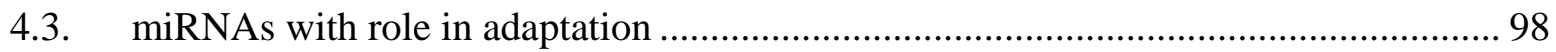

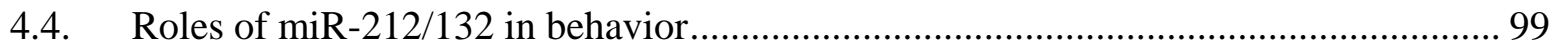

4.5. miR-212/132, neuronal networks and synaptic transmission ................................ 101

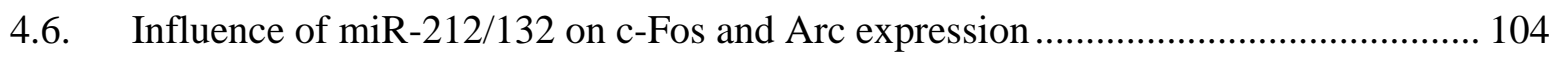

4.7. Functions of miR-212/132 at the molecular level.................................................... 105

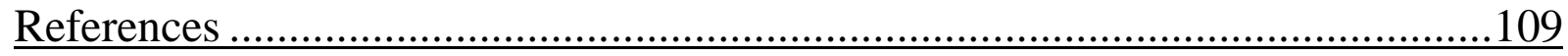

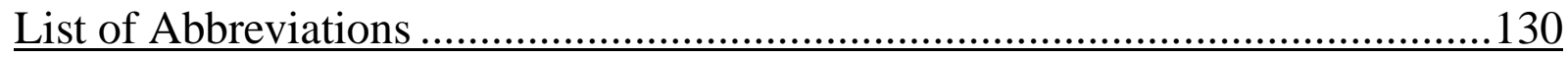

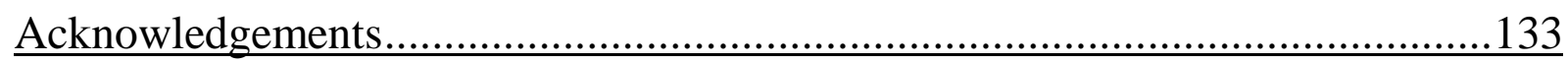

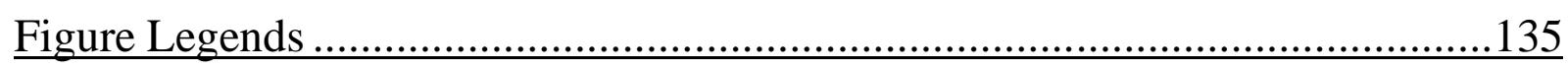

Table Legends........................................................................ 141

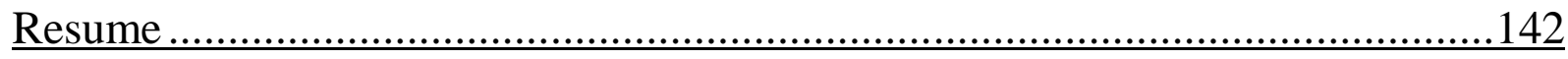




\section{ABSTRACT}

MicroRNAs are noncoding transcripts with crucial functions in probably every organ system. The roles of miRNAs in neuronal processes and cognitive functions are recently being appreciated. The discovery of neuronal activity regulated miR-212/132 family with their relatively high expression in brain, functional roles in synaptic remodeling and altered expression in disease states oriented special focus on their function in neuronal systems. In order to elucidate the in vivo functions of the miR-212/132, a mutant mouse line was generated via deletion of the genomic regions encoding pre-miR-212 and pre-miR-132 sequences. The $\beta$ galactosidase reporter gene knocked-in into the targeted locus in this mouse line allowed us to study the spatial expression pattern of $m i R-212 / 132$. B-galactosidase expression was detected in distinct layers of the cerebral cortex, CA3, CA1 and DG regions of the hippocampus in varying levels through its dorsal to ventral axis, as well as in lateral amygdala and striatum. The miR212/132 null mutant mice did not show any overt structural alterations in the brain, which led us to investigate possible phenotypes in the cognitive and electrophysiological functions of this mouse line.

Hidden platform water maze assay results revealed alterations in the spatial memory of the miR-212/132 $2^{--}$mice when compared with their WT littermates. In the open field test miR$212 / 132^{-/-}$mice were observed to be slightly hyperactive although they exhibited normal anxiety levels. The baseline excitatory synaptic transmission on Schaffer collateral synapses of the miR212/132 null hippocampus was normal. On the other hand the paired pulse ratios at different stimulus-intervals were higher in the miR-212/132 null hippocampus indicating alteration in the short-term synaptic facilitation. Moreover, long-term potentiation (LTP) on Schaffer collateral pathway, which is widely accepted as an assay modeling learning and memory, was enhanced in miR-212/132 $2^{-/-}$mice.

To elucidate the molecular mechanisms leading to the observed defects in the behavior and synaptic transmission of miR-212/132 $2^{--}$mice, temporal regulation of immediate early genes (IEG) were studied ex vivo. Arc, c-Fos and BDNF, which are the mediators of neuronal activity dependent changes in neurons, were downregulated in miR-212/132 $2^{-/-}$hippocampal neurons. Kainic acid treatment of neuronal cultures, which results in a robust neuronal activity, revealed a 


\section{Abstract}

phenotypical difference in the induction of IEG expression in miR-212/132 $2^{-/}$neurons. The relative levels of c-Fos, Arc and BDNF were similar in miR-212/132 $2^{-/}$and WT neurons upon incubation with kainic acid for 30 min or 3 hrs. In contrary the upregulation of c-Fos and Arc were significantly higher in miR-212/132/- neurons after 6 hrs of kainic acid treatment, which indicates that the loss-of-function of miR-212/132 alters the regulation of c-Fos and Arc upon longer neuronal induction.

Cyclic AMP response element binding protein (CREB) is a transcription factor that is phosphorylated after neuronal activation and subsequently mediates the expression of many downstream effector genes including c-Fos, Arc and BDNF. My results in this thesis have demonstrated that the loss of miR-212/132 function attenuates the phosphorylation of CREB in hippocampal neurons, and thereby providing evidence that miR-212/132 family is involved in the regulation of synaptic remodeling in hippocampal networks and consequently long-term spatial memory via regulating the CREB signaling which in turn influences the IEG expression. 


\section{INTRODUCTION}

\subsection{MicroRNA}

MicroRNAs (miRNA) are 20-24 nucleotide long non-coding RNAs with functions in almost every aspect of cellular metabolism ${ }^{1}$. Twenty years ago, the first miRNA, lin-4, was discovered in Caenorhabditis elegans ${ }^{2,3}$. It was recognized that lin-14 mRNA has sites at its 3' untranslated region (3'UTR) that is complementary to the lin-4. It has been already shown that the microRNA lin-4 represses the translation of lin-14 mRNA ${ }^{4}$. Negative regulatory sequences in the lin-14 3'-untranslated region are necessary to generate a temporal switch during C.elegans development. Therefore the role of this small RNA, which does not encode any protein, was shown to repress the translation of its target mRNA post transcriptionally by aligning with it.

After the initial discovery of miRNA, it took 7 years to first realize that the miRNAs are expressed in a variety of organisms ${ }^{5}$. Since then, via high throughput RNA sequencing, microRNAs have been detected in more than 206 different animal and plant species. There are 24521 hairpin precursor miRNAs expressing 30424 mature miRNAs, which have been discovered in various organisms ${ }^{6}$, including 1100 miRNAs in Homo sapiens, 717 in Mus musculus, 387 in Rattus norvegicus, 186 in Drosophila melanogaster and 233 in Caenorhabditis elegans ${ }^{7}$. Although miRNAs constitute $1-2 \%$ of genes in nematodes, insects and mammals ${ }^{8-10}$, more than $60 \%$ of protein coding genes are predicted as targets of the miRNAs ${ }^{11}$.

\subsection{1. miRNA sequence}

MiRNA-mRNA interaction is mediated by Watson-Crick base pairing. In plants, the complementarity of miRNA to its targeting mRNA is usually almost complete ${ }^{12}$. However, in metazoans, the alignment is generally imperfect, with some exceptions ${ }^{13-17}$. Specific 7 nucleotide long motifs in 3'UTRs of the mRNAs are crucial for the miRNA to bind and function. The complementary sequences of these binding motifs on the miRNAs are called "seed sequence" that determines the identity and targets of the miRNAs. The seed sequence starts at $2^{\text {nd }}$ nucleotide from the 5 , end of the mature miRNA and composed of 6-7 nucleotides ${ }^{18}$. Any mispairing in the seed sequence greatly interferes with the posttranscriptional repression. 
Mismatches or bulges in the central region of miRNA-mRNA heteroduplex $\left(\sim\right.$ between the $9^{\text {th }}$ and $12^{\text {th }}$ nucleotide), is important for the endonucleolytic cleavage of the mRNA. Moreover complementarity at $3^{\prime}$ half of the miRNA increases the stability of the duplex ${ }^{18,19}$. A miRNA does not necessarily bind only to the 3'UTR of its target. Some mRNAs have miRNA target sites within their 5'UTRs and coding sequences $(\mathrm{CDS})^{20}$. As another layer of complexity more than one kind of miRNA may bind to different parts of one transcript and silence its translation by acting in concert. Nanog is a good example for such a transcript. MiR-126 and miR-470 targets Nanog within its CDS while miR-134 binds to the site at 3'UTR. Those three miRNAs act cooperatively to regulate Nanog expression ${ }^{21}$.

The sequence of miRNA is not only important to determine its target but also matters for the stability of miRNA-mRNA duplex and the way of posttranscriptional repression ${ }^{22}$. The miRNA sequence also directs the localization of the miRNA and its turnover. MiR-29b contains a hexamer motif which mediates its nuclear import. Insertion of this sequence into another miRNA directs it into nucleus as well. MiR-29b might be sequestering its targets into nucleus and repress their translation by decreasing their concentration in cytoplasm ${ }^{23}$. MiRNAs are known to be relatively stable molecules with half-lives of 5 days ${ }^{24}$ to 2 weeks ${ }^{25}$. Interestingly the motif located at the 3' end of miR-382 is responsible for its instability in HEK293 cells ${ }^{26}$. The miRNA turnover can also be regulated by posttranscriptional-"postprocessing” modifications. For example uridylation of miR-29b and miR-29c in human cells and bantam (fly miRNA) at specific positions trigger their turnover ${ }^{27}$.

\subsection{2. miRNA Biogenesis}

A miRNA gene can be found as an independent genomic unit or might be located in an intron or exon of a protein-coding or non-coding genes ${ }^{28}$. They are usually transcribed by RNA polymerase II. MiRNAs that are located at downstream of tRNAs, Alu and other short interspersed nuclear elements were shown to be transcribed by RNA polymerase III $^{29-31}$. The transcript of a miRNA gene is known as primary miRNA (pri-miRNA) and contains a stem-loop structure that folds into hairpin structures with stem being composed of imperfect base pairs. A typical pri-miRNA is composed of stem segment which is $\sim 33 \mathrm{bp}$ long, terminal loop and flanking ends ${ }^{28}$. The pri-miRNAs show the properties of class II gene transcripts which are the 
3'poly(A) tail and 7-methyguanosine cap ( $\left.\mathrm{m}^{7} \mathrm{Gppp}\right)$ at 3' and 5' ends respectively ${ }^{32-38}$. PrimiRNAs does not necessarily encode single miRNA but can bear multiple miRNAs which may or may not be members of a functionally related miRNA family ${ }^{39}$.

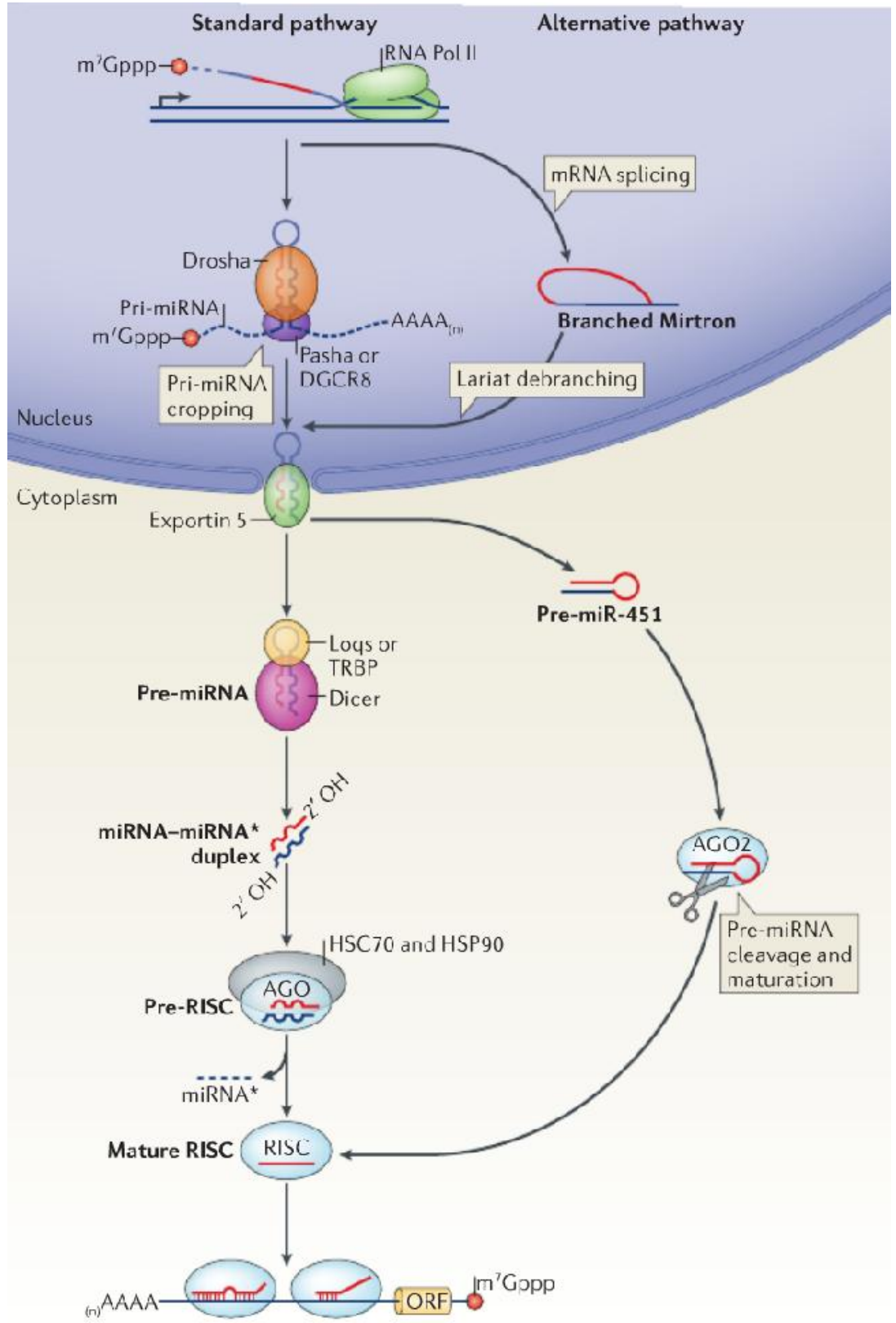

Figure 1: Illustration of microRNA Biogenesis Pathway (Ameres et al. 2013. Permission to reuse the illustration was given by Nature Publishing group. License \#:3297570668022) 
In the next step, the pri-miRNA, is further processed or trimmed into pre-miRNA in the nucleus. In the metazoans, RNase III enzyme called Drosha processes pri-miRNA into 60-70 nucleotide long precursor miRNA (pre-miRNA), which has a stem-loop structure ${ }^{40,41}$. Alternatively the pre-mRNA splicing mechanism, linearization by lariat debranching and 5'-to3' or 3'-to-5' trimming can generate pre-miRNA via bypassing the Drosha ${ }^{42}$. Drosha interacts with double stranded RNA binding protein DGCR8 in animals ${ }^{43}$ and Pasha in C.elegans ${ }^{44}$ which function as the cofactor for Drosha. This interaction defines the targets of the Drosha and increases its affinity to them. Moreover, DGCR8 precisely determine the cleavage site $^{22,45}$.

In animals, the cropping of pri-miRNA into pre-miRNA is mediated in the nucleus ${ }^{41,46}$. Then the pre-miRNA is exported into the cytoplasm by the Ran-GTP dependent nuclear transport receptor called exportin 5 through the nuclear pores ${ }^{47,48}$. The transport of the premiRNA takes place in a sequence independent manner ${ }^{49}$. However an efficient export requires RNA to have hairpin stem-loop structure with at least 16 bp and $\sim 2$ nucleotide 3' overhang ${ }^{50}$. This structure might be considered as a signature motif for exportin 5 to selectively export the pre-miRNA.

The exported pre-miRNA is recognized by another RNase III enzyme called Dicer and further cleaved into 22 nucleotide long miRNA-miRNA* duplex ${ }^{51-54}$. The $65 \AA$ distance between the PAZ domain, which is the RNA binding domain, and the catalytic site of Dicer corresponds to the length spanned by the miRNA-miRNA* duplex. Therefore it was claimed that Dicer acts as a ruler while determining the cleavage site ${ }^{55-57}$. However there are cases in which Dicer is not involved in the biogenesis of the miRNA. In zebrafish and mice, pre-miR-451 is not recognized by Dicer. Instead, it is further processed by AGO2 after being loaded into RNAinduced silencing complex (RISC) ${ }^{58-60}$.

The interaction partners of Dicer can determine its substrate specificity and modulate its activity. In flies, Loquacious-PA, -PB (Loqs-PA, Loqs-PB) and R3D1-L increases the Dicer1 specificity towards to pre-miRNAs ${ }^{61-63}$ while R2D2 that interacts with Dicer-2 restricts its activity on long dsRNA substrates ${ }^{64}$. TRBP in mammals and Loqs-PB in flies can change the site where the Dicer cleaves the pre-miRNA, which eventually gives rise to miRNAs with common precursor but different seed sequences. Those miRNAs with new target specificity are called isomiRs $^{65,66}$. 
Subsequently the miRNA-miRNA* duplex is loaded into Argonuate protein by HSC70HSP90 chaperone proteins ${ }^{67-71}$. Importantly the duplex is sorted in a specific way such that the correct strand can be selected. Therefore the selection does not take place in a random fashion. Otherwise the targeted gene set would be completely different. The strand which is incorporated into RISC complex is called "guide strand". The other strand is either unwounded and later on degraded or incorporated into RISC complex as well. It is called "passenger strand" or miRNA*. Thermodynamic properties, the AGO proteins within the RISC complex and the terminal nucleotide of miRNA-miRNA* duplex affect its sorting process. The guide strand in worms, fly and plants preferably have Uridine at their 5'ends while in human Uridine or Adenosine is favored $^{72-76}$. The RNA-protein interaction at Mid domain of AGO2 in human exclude Guanosine or Cytidine at 5' end of loaded miRNA. The nucleotide specific interaction is conserved in all types of human AGO proteins, AGO1 in flies and ALG-1 and ALG-2, which are miRNA acceptors in C.elegans ${ }^{74,75,77}$. Since the sites of cleavage on pre-miRNA, thus the location of 5' terminal nucleotide is determined by Dicer, it also determines later on the sorting features of the miRNA strands. This association implies a direct role of Dicer in the regulation of miRNA function $^{22}$. (Fig. 1)

The removal of miRNA* is the beginning of RISC maturation. Then the methyltransferase HEN1 methylates the AGO2 bound single stranded RNA which creates mature AGORISC complex ${ }^{78,79}$. Exceptionally there are some cases where miRNA* is also incorporated into RISC complex ${ }^{72}$.

\subsection{3. miRNA function}

The tight association between miRNA expression and diverse array of diseases reflects the significance of miRNA function ${ }^{80}$. They affect almost all cellular pathways, which are involved from development to oncogenesis. It is widely accepted that miRNAs regulate gene expression post-transcriptionally. However the detailed nature of the regulatory mechanism is still being debated. The target repression in plants are mostly mediated by endonucleolytic cleavage which is the result of extensive complementarity between the miRNA and the target mRNA $^{81-87}$. Blockage of translation was also observed in plants ${ }^{88,89}$. Oppositely, in animals miRNA-target base pairing is generally imperfect, this is therefore not sufficient to mediate 
mRNA cleavage. Although there are some examples of perfect miRNA-target match, it is not clear whether there is decrease in the levels of the target transcript in those cases ${ }^{16,17,90,91}$. It is known that in metazoans, the translational control is mostly mediated by the block of ribosome or deadenylation of $\mathrm{mRNAs}^{1}$. On the other hand, more than $84 \%$ of the miRNA-mRNA interaction that leads to decreased protein translation is attributed to decreased mRNA level in mammalian cells ${ }^{92}$. This suggests that the translational repression mechanisms which are distinct from endonucleolytic cleavage eventually also give rise to mRNA destruction ${ }^{22}$.

Taken together, it is obvious that miRNAs regulate the expression of their hundreds of target genes through translational repression. However there are also cases that miRNA activity increases expression of its target. For example, miR-373 enhances the transcription of E-cadherin and cold-shock domain containing protein C2 (CSDC2) by targeting specific sequence at their promoters $^{93}$. Hepatitis C virus requires endogenous miR-122 for enhancement of internal ribosomal entry site (IRES) directed translation and efficient viral replication ${ }^{94,95}$. Under optimal conditions miR-369-3 binds to 3'UTR of tumor necrosis factor- $\alpha$ (TNF- $\alpha$ ) and represses its translation. However upon serum starvation, miR-369-3 mediates an increase in TNF- $\alpha$ levels by recruiting AGO2 and fragile $\mathrm{X}$ mental retardation related protein 1 (FXR1) ${ }^{96,97}$. MiR-10a enhances the translation of ribosomal protein genes by binding to the 5'TOP motif at downstream of the transcript which thereby can control the global protein synthesis ${ }^{98}$.

\subsubsection{Regulation of miRNA activities}

MiRNAs are regulatory elements. However, their activity is also subject to regulation. Spatiotemporal regulation of miRNA activity is especially important in neurons, where the expression of learning and memory related genes are regulated at the correct time and at correct neuronal compartment. As a good example, miR-134 inhibits Lim-domain-containing protein kinase 1 (LIMK1) translation at synapses. LIMK1 is a kinase that predominantly found in neurons. It regulates actin filament dynamics by phosphorylating ADF/cofilin family proteins ${ }^{99}$. The miR-134 overexpression and Limkl knockout mice show similar alteration in dendritic spine structure $^{100}$. When cultured neurons are exposed to brain derived neurotrophic factor (BDNF), the miR-134-mediated repression is relieved which leads to synaptic remodeling ${ }^{101}$. MiR-138 which is expressed in fly olfactory neurons and rat hippocampal neurons suppresses translation 
of APT1 at dendritic spines ${ }^{102}$. When there is a neuronal stimulation, RISC assembly factors Armitage (in fly) and MOV10 (in rat) are degraded which abolishes the miRNA meditated suppression ${ }^{103,104}$. Fragile X mental retardation 1 protein (FMRP) is known to be a translational repressor $^{105,106}$. MiR-125b, which interacts with FMRP suppresses NMDA receptor subunit NR2A. The effect of miR-125b overexpression can be rescued by depletion of FMRP, which suggests that the NR2A silencing is mediated by FMRP-miR-125b synergy ${ }^{107}$.

Similar to the protein coding genes, the miRNA genes are also under the control of cis and trans acting regulatory elements ${ }^{34}$. The promoters of the miRNA genes also contain TATA box sequences, $\mathrm{CpG}$ islands and are subjected to histone modifications. Therefore it is expected that the microRNA encoding genes are under the control of transcription factors, enhancers, histone modifying enzymes and epigenetic modifications ${ }^{35}$. For example p53 enhances the transcription of miR-34 and miR-107 which is required for the cell cycle arrest and apoptosis. RE1 silencing transcription factor (REST) silences miR-124a expression in non-neuronal cells and neuronal progenitors probably by recruiting histone deacetylases to the promoter of miR124a gene ${ }^{108}$. It was shown that $10 \%$ of miRNA genes were misregulated when DNMT1 and DNMT3b methyltransferases were knocked out ${ }^{109}$.

\subsection{MiR-212/132}

MiR-212/132 family is comprised of miR-212 and miR-132, which are located on the same primary transcript. MiR-212 was firstly identified in HeLa cells ${ }^{110}$. MiR-132 was initially isolated from adult mouse brain tissue and defined as predominantly brain specific ${ }^{111}$. MiR212/132 cluster is found on chromosome 17 in human, 11 in mouse and 10 in rat. It was detected in many organisms including Gallus gallus, Xenopus tropicalis, Monodelphis domestica, Macaca mulatta, Eguus caballus, Bos Taurus, Tetraodon lineatus, Canis lupus familiaris, Rattus norvegicus, Mus musculus, Macaca mulatta, and Homo sapiens which suggests that the gene family is highly conserved in vertebrates.

\subsection{1. miR-212/132 gene family structure and expression}

MiR-212 is located upstream of the miR-132 in the primary transcript. Pre-miR-212 and pre-miR-132 encoding sequences are separated by only 203 nucleotides. The stem-loop structure 
of pre-miR-132 and pre-miR-212 are 66 and 90 nucleotides long respectively. Mature miR-132 and miR-212 molecules are composed of 22 and 21 nucleotides respectively ${ }^{112}$. The sequences of mature miR-132 and miR-212 are highly similar with only 4 nucleotide differences in their 3 'part and an additional nucleotide in miR-132 sequence. They have a conserved 'seed' region, which suggests that they both target same set of genes although with variable affinities due to differences on their 3' side. However it is known that considering only the complementarity of the seed sequence in target recognition is not always accurate, since the level of complementarity at the 3' side of the miRNA also influences the targeting efficiencies based on the stability of the predicted miRNA-mRNA binding ${ }^{113,114}$.

MiR-212 and miR-132 can be classified as neither intronic nor exonic miRNA. Two transcript variants in miR-212/132 locus were detected in different tissues ${ }^{115-117}$. In the variant 1 , the miR-212 and miR-132 encoding sequences are located between two exons of a yet another non-coding gene ${ }^{116,117}$. However miR-212 and miR-132 are located in one of the exons of the variant 2 . The variant 1 was detected in brain and testis, while variant 2 is expressed in brain, testis, heart and in epithelial and stromal fractions of mammary gland ${ }^{115}$. So far the transcript (DQ223059 in rat and AK006051 in mouse) encoded from the miR-212/132 loci were not shown to be translated. The transcript is claimed to be a non-coding RNA since it does not have an open reading frame.

MiR-212/132 locus encodes 4 miRNAs; miR-212, miR-132, and the miRNAs that arise from their complementary strands; miR-212* and miR-132*. BDNF treatment of cortical neurons induces the expression of miR-212, miR-132 and also the corresponding star sequences. However the levels of miR-132 is higher than miR-212 and miR-132*, while the expression levels of miR-212 and miR-212* are similar to each other ${ }^{117,118}$. The seed sequences of miR212* and miR-132* are completely different (Fig. 2). Their detection by quantitative RT-PCR in cell culture systems suggests that they should be protected from degradation and may have some functional roles in these cells. However so far no experimentally confirmed functions of miR$212 *$ and $\mathrm{miR}-132 *$ have been reported. 
1. Introduction

a

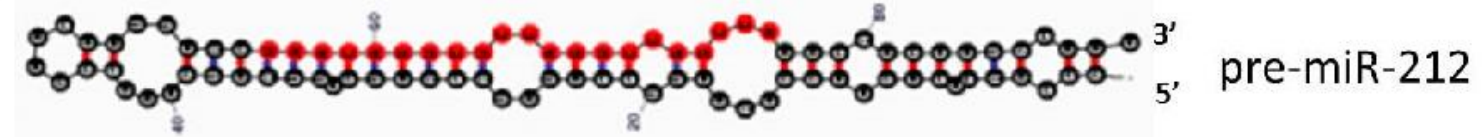

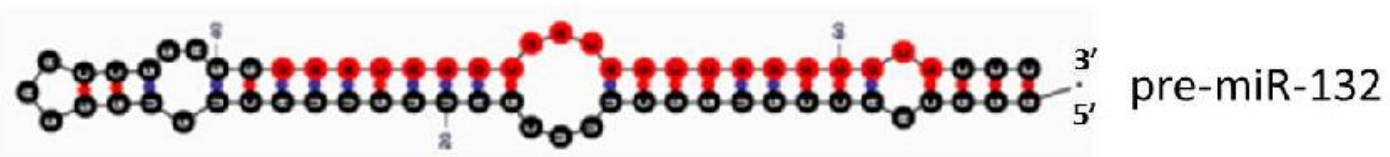

b

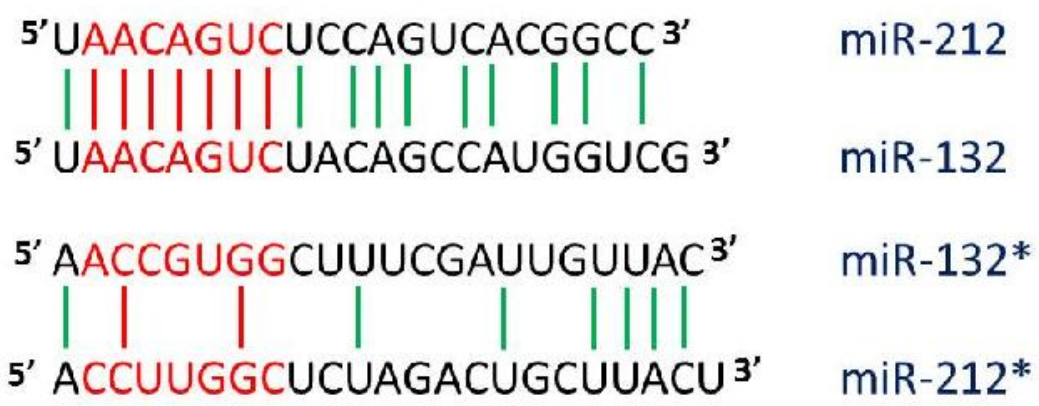

Figure 2: miR-212/132 sequence and structures. a) The structure of pre-miR-212 and pre-miR-132 is depicted. The region, which will become mature miRNA are shaded in red. b) The mature miR-212, miR-132, miR-212* and miR-132* sequences are shown. The seed sequences are typed in red.

In the mouse brain, primary transcript levels of miR-212/132 and mature miR-132 levels increase with age. As detected by qPCR, miR-132 expression in the hippocampus, striatum and olfactory bulb of developing mice is first observed at birth and then progressively increases during postnatal life and reaches to adult levels at 3 weeks of age ${ }^{119,120}$. The miR-212/132 loci bears 4 cyclic AMP response element (CRE) binding sites of which two are located at upstream of miR-212 and the other two are between miR-212 and miR-132 sequences ${ }^{121}$. Cyclic AMP response element binding protein (CREB) controls the expression of miR-212/132 by binding to consensus CRE sequences in neurons ${ }^{116,117,122,123}$. They were attributed as neuronal activity regulated miRNAs since the expression of miR-132 was shown to be induced by treatments with forskolin, NMDA, $\mathrm{KCl}$ or bicuculline in neuronal cultures ${ }^{116,117,119,123-125}$. High frequency stimulation (HFS) in vivo and injection of epileptic seizure inducing drugs, pilocarpine and kainic acid, increases the pri-, pre- and mature-miR-132 levels in hippocampus. Cocaine was also found to be an inducer of miR-132 expression in the hippocampus and cerebellum ${ }^{120,122,126,127}$. Moreover BDNF induces miR-212/132 transcription in neurons while basic fibroblast growth factor (bFGF) mediates the upregulation of miR-132 expression in both neurons and astrocytes. ${ }^{116,128,129}$. 
MiR-212/132 locus is also subjected to transcriptional repression by repressor element 1 silencing transcription factor (REST). There is one REST binding site between miR-212 and miR-132 coding sequences. Expression of dominant-negative REST mutant in mouse embryonic fibroblasts (MEFs) leads to an increase in pre-miR-132 levels while ectopic expression of wildtype REST in cortical neurons downregulates the levels of pre-miR-132 transcript within 48 hours ${ }^{108}$.

As stated above neuronal activity inducing and neuromodulatory agents influence the expression of miR-212/132 in vitro and in vivo. However treatments with these agents represent an artificial method of neuronal stimulations. The natural course of neuronal stimulation generally requires an exposure to a neurostimulatory chemokine or another stimulated cell. Exploring a novel environment, experiencing fear or activation of sensory systems such as visual, auditory or tactile systems, activates various neuronal pathways and initiate new transcriptional programs. Seeing, hearing or tasting activates many neuronal pathways and initiate new transcriptional programs. MiR-212/132 transcription was also shown to be experience dependent. The levels of pri- and mature miR-132 in the visual cortex of postnatal mice increased as the mice become adolescent. Interestingly this increase in the RNA levels was abolished when the mice were reared continuously in dark throughout their juvenile life. It was concluded in that study that monocular deprivation decreases the mature miR-132 levels. However when the eyes are exposed to light again, miR-132 levels increases back to the normal levels ${ }^{130,131}$. Expression of miR-132 in suprachiasmatic nucleus (SCN) also depends on the exposure to light and assigns it as an important modulator of circadian clock ${ }^{123,132}$. It was also shown that exploring behavior in Barnes maze increases the miR-212/132 expression in CA1 and CA3 regions and granule cell layer $(\mathrm{GCL})^{133}$. When the mice were exposed to an odorant, the upregulation of pri-miR-132 level was observed within 15 minutes and reached to its highest levels in 45 minutes. Additionally exploring a novel environment and fear conditioning also induced pri-miR-132 transcription $^{120,134}$

In the context of miRNA dependent regulation of gene expression, the catabolism of miRNAs is as important as the regulation of their biogenesis. It was shown that the neuronal activity dependent miRNA turnover is a common feature of neuronal miRNAs. In cultured hippocampal neurons, the turnover of miR-124, -128, -134 and -138 initiates soon after the cells 
are exposed to glutamate. However when the neuronal activity is suppressed by application of 6nitro-2,3-dioxo-1,4,-dihydrobenzo[f]quinoxaline-7-sulfonamide (NBQX), which blocks $\alpha$ amino-3-hydroxy-5-methyl-4-isoxazolepropionic acid receptor (AMPAR), kainate receptors and $( \pm)$-3-(2-carboxycpiperazin-4-yl)propyl-1-phosphonic acid that blocks N-Methyl-D-aspartic acid receptor (NMDAR), no significant change in the levels of miR-124, -128, -134 and -138 was observed. Interestingly under those conditions where neuronal activity is blocked, the levels of miR-132 progressively declines and reaches to a significantly low level within half an hour ${ }^{135}$.

Structurally different non-coding transcripts, which are expressed from the miR-212/132 gene family loci, give rise to miR-212 and miR-132. In the neuronal systems, the regulation of the miR-212/132 gene is influenced by the neuronal activity. Pharmaceutical activation of neurons in vivo and in vitro induces miR-212/132 transcription. Besides sensory stimulation and exploratory behavior enhances the gene transcription as well. CREB, REST and some epigenetic modifications are known to be involved in the regulation of miR-212/132 transcription. However still there is no clear picture regarding the spatiotemporal regulation of miR-212/132 expression in vivo. Moreover the mechanism of differential expression of miR-212 and miR-132 is not known.

\subsection{2. miR-212/132 function}

Most of the miR-212/132 functional studies have been done in vitro and there are only a few reports of miR-212/132 function using a knockout mouse model. Twenty molecular targets of miR-212/132 have been validated experimentally up to now since first experimental detection of miR-132 in $2002^{136}$ (Table 1). The role of miR-212/132 was reported in neuronal morphogenesis, drug addiction, newborn neuron integration, cognitive functions, ocular dominance plasticity, circadian rhythmicity, mammary gland development, cardiac hypertrophy, angiogenesis and immune functions ${ }^{136,137}$. Correlation between some neurological disorders and alteration in the levels of miR-212/132 were also detected. Considering that miRNA target prediction tool TargetScan predicts 314 putative targets of miR-212/132 with highly conserved sites, it is very likely that there are still yet a large number of unknown molecular functions of miR-212/132 waiting to be revealed in future studies. 
Table 1: Experimentally confirmed targets of miR-132 and miR-212.

\begin{tabular}{|c|c|}
\hline Target & Targeted by \\
\hline Heparin-binding EGF-like growth factor (HB-EGF) ${ }^{138}$ & miR-132 and miR-212 \\
\hline Methyl CpG binding protein $2(\mathrm{MeCP} 2)^{125,132}$ & miR-132 and miR-212 \\
\hline Matrix metalloproteinase 9 (MMP9) ${ }^{115}$ & miR-132 and miR-212 \\
\hline Retinoblastoma tumor suppressor $1(\mathrm{Rb} 1)^{139}$ & miR-132 and miR-212 \\
\hline Cardiac L-type $\mathrm{Ca}^{2+}$ channel $\beta 2$ subunit protein ${ }^{140}$ & miR-132 \\
\hline Acetylcholinesterase $(\mathrm{AchE})^{141}$ & miR-132 \\
\hline Jumonji, AT-rich interactive domain 1A (JARID1A) ${ }^{132}$ & $\operatorname{miR}-132$ \\
\hline RAS P21 Protein Activator (p120RasGAP) ${ }^{142}$ & miR-132 \\
\hline Rac GTPase activating protein (p250GAP) ${ }^{116,119,143}$ & miR-132 \\
\hline E1A binding protein p300 (p300) ${ }^{132,144}$ & miR-132 \\
\hline Polyadenylate-binding protein- interacting protein $2(\mathrm{PAIP} 2 \mathrm{~A})^{132}$ & miR-132 \\
\hline Polypyrimidine tract binding protein $2(\mathrm{PTBP} 2)^{145}$ & $\operatorname{miR}-132$ \\
\hline Regulatory factor X, 4 (RXF4) ${ }^{123}$ & $\operatorname{miR}-132$ \\
\hline Sirtuin $1(\text { SirT1) })^{146}$ & miR-132 \\
\hline Signal transducer and activator of transcription 4 (STAT4) ${ }^{147}$ & $\operatorname{miR}-132$ \\
\hline B-cell translocation gene $2(\mathrm{BTG} 2)^{132}$ & miR-132 \\
\hline Nuclear receptor subfamily 4 group A member 2 (Nurr1) ${ }^{148}$ & $\operatorname{miR}-132$ \\
\hline Sprouty-related EVH1 domain containing 1 (SPRED1) ${ }^{122}$ & $\operatorname{miR}-212$ \\
\hline Zonula occludens $1(\mathrm{ZO}-1)^{149}$ & $\operatorname{miR}-212$ \\
\hline Phosphoprotein enriched in astrocytes 15 (PED/PEA-15) ${ }^{150}$ & miR-212 \\
\hline
\end{tabular}

\subsubsection{1. miR-212/132 in neuronal morphogenesis}

Ectopic expression of miR-132 in cortical neurons increases the total neurite length and the number of neurite outgrowths while blocking miR-132 activity with antisense 2' O-methyl RNA attenuates the neurite sprouting ${ }^{116}$. MiR-132 targets p250GAP, which is a brain enriched GTPase activating protein ${ }^{151}$. P250GAP enhances intrinsic GTPase activity of GTP-binding proteins which carries them from GTP-bound active state to GDP-bound inactive state ${ }^{152}$. As primary hippocampal neuron culture gets mature, the levels of miR-132 and the spine density on their dendrites increase in parallel. Interestingly the miR-132 and the p250GAP levels show reverse correlation at different day in vitro (DIV) stages of hippocampal neuron cultures ${ }^{119}$. 
When p250GAP is silenced by shRNA, the neurite sprouting is enhanced in a similar way as observed after miR-132 overexpression ${ }^{116}$. Moreover, when neuronal cultures are exposed to bicuculline, which is a GABA receptor antagonist used to lower the action potential threshold, the spine density on dendrites, the width and size of spine heads increase. The observation of the similar phenotype upon miR-132 expression and p250GAP silencing reveals the association between neuronal activity, miR-132 expression and $\mathrm{p} 250 \mathrm{GAP}$ repression ${ }^{119}$.

Rho family of small GTPase Rac1, and Rac guanine exchange factor (Rac GEF) Kalirin7 function in the downstream of miR-132-p250GAP pathway. Rac1, which is inactivated by p250GAP, has a functional role in the stability of dendritic spines. ${ }^{151,153,154}$. Kalirin-7 is an alternatively spliced form of kalirin ${ }^{155}$. It is expressed in spines and regulates the spine formation $^{156-158}$. Kalirin-7 can rescue the phenotype caused by miR-132 inhibition, thereby suggesting that the neuronal activity dependent spine formation should be dependent on both p250GAP repression by miR-132 and kalirin-7 mediated Rac1 activation ${ }^{119}$.

There are inconsistent and controversial findings among different studies regarding the effect of miR-132 overexpression and deletion. Edbauer et al. showed that miR-132 overexpression in hippocampal neuronal cultures leads to a decrease in spine density and an increase in spine width with no effect on the spine length. They also demonstrated that knocking down the miR-132 levels by sponging does not influence the spine length, density or width. It rather only causes a decrease in the primary dendrite ramification and the area covered by dendritic branches ${ }^{107}$. Siegel et al. however, reported decreased spine volume upon transfection of neurons by miR-132 antisense RNA ${ }^{102}$. In the study of Mellios et al., lentiviral infection of V1 cortical neurons at layer 2/3 with miR-132 sponge reduced the spine density and the number of mushroom spines while increasing the abundance of filopodia. The size of the dendrites of the sponge infected neurons remained normal ${ }^{130}$. On the other hand, Hansen et al. demonstrated that the dendrites of CA1 pyramidal neurons of miR-132 overexpressing mice have higher spine density compared to control mice ${ }^{133,159}$. Magill et al. analyzed the newborn neurons of adult hippocampus and revealed that genetic deletion of miR-212/132 leads to decrease in spine density, dendrite length and arborization ${ }^{118}$. Luikart et al. showed that inhibition of miR-132 in newborn neurons at subgranular zone by retroviral infection of sponge vector only decreased their spine density at 21 days post infection (DPI ${ }^{160}$. Pathania et al. infected newborn neurons 
with retrovirus carrying miR-132 sponge at subventricular zone. They have observed a decrease in dendritic complexity, dendrite length and spine density. When miR-132 was overexpressed spine density, dendritic complexity and spine length was increased ${ }^{161}$. Remenyi et al. reported another contradictory in vivo neuronal morphology data such as that they did not observe any difference in the spine density in CA1 neurons of conventional miR-212/132 knockout mice. However the cultured cortical neurons obtained from miR-212/132 knockout mice showed a rather small decrease in the dendrite length and branching after 24 hour in culture. Interestingly this difference disappears at 48 hours of culturing ${ }^{162}$.

The studies explained above are obviously not demonstrating consistent results. Therefore further studies are necessary in order to elucidate the functional roles of miR-212/132 on neuronal morphogenesis.

\subsubsection{2. miR-212/132 in newborn neuron integration}

Neurogenesis does not only take place during the development of the nervous system but also required for the homeostasis of the nervous system throughout the whole life of the organism. There are two sites known where neurogenesis takes place in the adult brain; subventricular zone (SVZ) of the lateral ventricles and subgranular zone (SGZ) of the dentate gyrus (DG). The newborn neurons in the SVZ migrate into the olfactory bulb through rostral migratory stream (RMS) and differentiate into the periglomerular and granule neurons. New neurons generated within the SGZ differentiate in order to become dentate granule neurons which later incorporate into the granule cell layer of dentate gyrus ${ }^{163}$.

\subsection{Neurogenesis at SGZ}

MiR-132 is not expressed by the neuronal progenitors in DG. However the newborn neurons at SGZ start to express miR-132 upon differentiation and as the neurons get mature the expression levels then progressively increase. The knockdown of miR-132 by specific sponge vector in newborn neurons can be achieved by retroviral transduction in vivo. The frequency of spontaneous excitatory postsynaptic current (sEPSC) was decreased substantially in neurons after miR-132 knockdown although the amplitude was not affected. Paired recordings from the miR-132 sponge transduced or control neurons upon stimulation of the perforant path revealed 
that evoked EPSC was smaller in sponge expressing neurons. However, paired pulse ratio was not affected upon miR-132 knockdown ${ }^{160}$. These findings suggest that although miR-132 loss-offunction does not result in a change in the neurotransmitter release probability, it may lead to a decrease in the synapse amount or in AMPAR levels found at individual synapses. Therefore miR-132 is essential for the proper integration and functioning of newborn neurons. Interleukin6 (IL-6) was found to be increased in the neurons upon miR-132 knockdown. Since IL-6 is known to block the neuronal differentiation via its effect on neural stem cells ${ }^{164,165}$, increase in IL-6 upon miR-132 inhibition may explain the observed impairment of the neurogenesis.

\subsection{Neurogenesis at SVZ}

Similar to neurogenesis at SGZ, the neurons generated by neuronal stem cells at SVZ also initiates the miR-132 expression only when newborn neurons starts to differentiate and to be integrated into the neuronal networks within the olfactory bulb. If the miR-132 function is blocked in newborn neurons at SVZ by in vivo neonatal electroporation of specific sponge vector, these neurons show a decreased frequency and amplitude of EPSCs but normal GABAergic PSC. On the other hand, the overexpression of miR-132 increases the frequency, but not the amplitude of GABAergic currents.

In the natural course of adult neurogenesis, half of the newborn neurons die within the first 15-45 days. Their survival depends on the neuronal input they receive ${ }^{166}$. If the ectopic miR132 overexpression is mediated in neuroblasts at the time of their birth, the number of cells that express miR-132 is decreased by $34 \%$. Since more activated caspase-3 was detected in miR-132 overexpressing neuroblasts at RMS, it is claimed that the decrease is due to increase in apoptosis of neuroblasts. However when the overexpression is induced in the neuroblasts 7 days after birth of mouse, the survival rate of newborn neurons increases ${ }^{161}$. These results indicate the spatiotemporal regulation of the miR-132 expression is important for the synaptic integration and survival of newborn olfactory bulb neurons.

\subsubsection{3. miR-212/132 in dopaminergic neuron differentiation}

Dopaminergic neurons are mainly found in the midbrain ${ }^{167}$. They function in motor control, reward system, motivation and emotional behavior ${ }^{168}$. Degeneration of dopaminergic 
neurons is implicated as the basis of Parkinson's disease (PD) ${ }^{169}$. Stem cell therapy to replace the dopaminergic neurons is considered as a promising therapeutic option against $\mathrm{PD}^{170}$. Therefore it is crucial to understand the mechanisms of dopaminergic neuron differentiation. MiR-132 was found to be also involved in this process. The overexpression of miR-132 decreases the rate of differentiation while blocking the miR-132 activity augments the differentiation of dopaminergic neurons. Nurr1 is an essential transcription factor for the induction of dopaminergic neuron differentiation $^{171}$. MiR-132 was shown to regulate the expression of Nurr1. Although the mechanism is not clear, it was claimed that the homeostatic interaction between miR-132 and Nurr1 might be important for the regulation of dopaminergic neuron differentiation ${ }^{148}$.

\subsubsection{4. miR-212/132 in synaptogenesis}

\subsection{Synaptic transmission}

Impey et al. showed that the inhibiton of miR-132 in hippocampal neurons by antisense RNA reduced the miniature excitatory postsynaptic current (mEPSC) frequency by $30 \%$ without affecting its amplitude, rise or decay time ${ }^{119}$. MiR-132 inhibition downregulated the surface expression levels of GluR $1^{119}$. In contrary Edbauer et al. demonstrated that miR-132 silencing by sponging does not affect mEPSC amplitude or frequency. In vivo miR-132 sponging experiments showed no change in the mEPSC frequency of V1 cortical neurons at layer $2 / 3$ while the amplitude has decreased. ${ }^{130}$ Oppositely miR-132 overexpression increases the mEPSC amplitude and frequency ${ }^{107}$. On the other hand the analysis performed on autaptic hippocampal neurons by Lambert et al. showed that lentiviral overexpression of miR-132 does not cause any change in size or frequency of mEPSC. The paired-pulse ratio was enhanced and the synaptic depression amount was decreased. Those phenotypes were not due to the alteration in basal synaptic transmission, change in the amount or rate of refilling of readily releasable pool (RRP) of synaptic vesicle size, calcium dependence of the synaptic release or postsynaptic receptor desensitization $^{172}$. Furthermore the EPSC was not different in miR-132 overexpressing neurons. Size of a postsynaptic response to a single nerve impulse depends on the probability of presynaptic vesicle release, quantal response to a single vesicle and the number of synapses ${ }^{173}$. Therefore it is possible to conclude that miR-132 overexpression does not change the presynaptic vesicle release $^{172}$. 
Being familiar to an object is a component of visual recognition memory. The acquisition and consolidation of the memory is dependent on synaptic plasticity in perirhinal cortex $(\mathrm{PRh})^{174-177}$. Scott et al. reported that lentiviral overexpression of miR-132 in PRh of mice does not affect the baseline synaptic transmission. Induction of long-term potentiation (LTP), which is considered as representative of molecular and cellular aspects of learning and memory ${ }^{178}$, resulted in no difference between control group and miR-132 overexpressing PRh slices in terms of synaptic strength following high frequency stimulation (HFS). Long-term depression (LTD) is defined as the long lasting weakening of synaptic connections. It is an important molecular and cellular mechanism for modulation of neuronal networks, thereby the neuronal plasticity ${ }^{178}$. The chemical induction of LTD is possible by treatment with carbachol (CCh) that activates muscarinic acethylcholine (Ach) receptors ${ }^{179}$. CCh treatment on PRh slice of miR-132 overexpressing mice caused higher depression of synaptic transmission compared to control group. Moreover, following the washout of CCh, although field EPSC (fEPSC) amplitudes came back to normal levels in the control group, the depression stayed significantly higher in miR-132 overexpressing slices ${ }^{179}$. Those findings suggest that miR-132 modulates the level of synaptic depression in $\mathrm{PRh}$ slices without affecting the basal synaptic transmission.

In vivo loss-of-function study performed by Remenyi et al. revealed interesting functional roles of miR-212/132 family during synaptogenesis. The basal synaptic transmission is decreased in the Schaffer collateral pathway of miR-212/132 knockout mice. However no difference was observed in paired pulse ratio between miR-212/132 mutant and control groups, which suggests that there is no alteration in synaptic vesicle release probability. LTP was observed to be higher in miR-212/132 mutant hippocampi. Interestingly LTP was observed to be lower in the neocortex of mice. Additionally $40 \%$ decrease in the amplitude and size of the mEPSC was observed in the neocortex upon miR-212/132 deletion.

The studies on the roles of miR-212/132 in synaptic transmission are either not supportive or contradicting with each other. As explained in the section 1.2.2.1 the research about the influence of miR-212/132 on neuronal morphology revealed contradicting results as well. Since it is known that the structural plasticity and synaptic transmission is highly dependent on each other ${ }^{180,181}$, having contradictory results may also have same reasons in both cases (for 
further discussion see the section 4.2). Therefore the experimental results should be evaluated individually by considering each parameter that potentially influences the outcome.

\subsection{Ocular dominance plasticity}

Complex instinctual behaviors of animals are shaped under influence of genetic and environmental factors. The characteristics of repertoire of behaviors like mating, fighting, foraging, curiosity or anxiety is dependent on patterns of connectivity in the brain of individual. The development of the neuronal networks are indeed influenced by the environment, especially during the early periods of life, which are called critical periods. The critical period for zebra finches to learn how to tweet is the first 100 days of their life ${ }^{182}$. One can learn a foreign language and speak it as fluently as native speaker if $s /$ he learned it till the age of seven ${ }^{183}$. Critical period in the visual system development was well studied in monkeys, cats, rats and mice. The plasticity of afferents, which reach to visual cortex, changes their pattern of connectivity depending on the light stimulus received by each of the two individual retinas. This phenomena is called ocular dominance plasticity and considered as a good model to investigate synaptic plasticity under different circumstances ${ }^{184}$. It has been demonstrated that the distributions of neurons, which receive signal from one of the eyes or both show Gaussian distribution. This means that most of the neurons can receive signal from both retinas while relatively lower amount of cells receive input from one of the retinas. When one eye is sutured, the distribution of activated neurons is shifted towards the neurons that receive signal from the intact eye. If the deprivation is performed during the critical period, the ocular dominance is altered irreversibly $^{185-187}$.

As an important regulator of synaptic plasticity, the functional role of miR-132 in ocular dominance was also recently demonstrated. It has been shown that miR-132 inhibition by lentiviral transduction of miR-132 sponge into V1 neurons abolishes the ocular dominance shift, which normally is observed 4 days after the monocular deprivation ${ }^{130}$. As explained above, the miR-212/132 expression in visual cortex neurons is dependent on the neuronal stimuli supplied by light exposure. When the downregulation of the miR-132 levels upon monocular deprivation is restored by miR-132 mimic infusion, the shift in the ocular dominance plasticity is prevented 
as well ${ }^{131}$. Those findings suggest that proper spatiotemporal regulation of miR-212/132 is essential for the ocular dominance plasticity.

Furthermore light exposure leads to histone modifications at CRE binding sites of miR212/132 loci. Phosphorylation of serine 10, acetylation of lysine 14 and demethylation of lysine 4 on histones are the observed modifications which are associated with light exposure and thought to be the epigenetic changes that induce miR-212/132 expression ${ }^{131}$.

Different studies revealed the role of miR-212/132 in the modulation of neuronal networks and the differential regulation of the miR-212/132 loci upon neuronal activity. The research on the role of miR-212/132 in ocular dominance plasticity also revealed that the ability of environmental factors to modulate the neuronal networks is dependent on proper regulation of miR-212/132 expression.

\subsubsection{5. miR-212/132 in behavior}

Response to external stimuli is a common inheritable feature of all living organisms. Behavior is an array of responses that living organisms execute depending on the various internal or external stimuli. Organisms learn and recall what they have experienced in order to be able to accurately and appropriately respond under different circumstances. This allows them to increase their chances of survival. Thus, they regulate their inner homeostasis depending on external cues, like light, abundance of food or heat. Organisms must have motivations to perform an action and to keep that action sustainable which would be crucial for their survival like feeding or reproduction. Alteration in any of those mechanisms would be reflected as a change in the behavior of the organism.

\subsubsection{1. miR-212/132 in learning and memory}

Learning is a behavioral process of skill or knowledge acquisition through experience. The storage of the acquired information is called memory. The stored information in the memory can be recalled which in turn may cause a change in the behavior of the organism. Brain is a complex adaptive system which undergoes modifications at cellular and subcellular level during the process of learning and memorization. As explained above, miR-212/132 regulates various 
aspects of synaptogenesis. Therefore it is likely that this gene family is also involved in learning and memory.

Using novel object recognition test, Hansen et al. studied the visual recognition memory of the transgenic mice overexpressing miR-132 specifically in the adult forebrain neurons ${ }^{159}$. In the training phase of this behavioral assay, the transgenic and control mice were allowed to explore two identical objects for a certain time and then placed back in their home cage for 30 min. In the test phase, one of these objects was replaced with a novel but similar object and the time spent by mice on each object was measured. Although mice in the control group expectedly spent more time to explore the novel object, the transgenic mice spent equal amount of time on both objects. This finding suggests that miR-132 overexpression impairs short-term visual recognition memory.

Lentiviral mediated overexpression of miR-132 in PRh altered specifically the short-term visual recognition memory ${ }^{188}$. These miR-132 overexpressing mice spent less time exploring the novel object compared to the control mice when the test was performed 20 min after the habituation. However no impairments could be observed when mice were tested 24 hours after the habituation.

Barnes maze assay measures the ability of mice to learn, memorize and recall the longterm memories of spatial cues ${ }^{189}$. In this assay system, mice are placed on a brightly illuminated round table that has 18 evenly spaced holes. One of the holes let the mice to escape into a dark box, which is considered by mice as a safe place to stay rather than to be on the open and bright surface of the table. After several training sessions, mice are expected to find the correct hole in a shorter time and with less trial errors. Two-fold induction of miR-132 expression in the adult forebrain neurons improved the performance of transgenic mice in Barnes maze assay. Compared with the control group, the escape latency and the trial errors were lower in the group of transgenic mice between the $2^{\text {nd }}$ and $4^{\text {th }}$ days of trials. On the $5^{\text {th }}$ day both the scores became similar between transgenic and control groups. However if the miR-132 expression was induced by approximately around 4-folds in the adult forebrain neurons, the performance of these transgenic mice in this behavioral assay become poorer in comparison with the control group ${ }^{133}$. Therefore maintaining optimal levels of miR-132 expression in forebrain neurons is crucial for the establishment of the proper spatial memory. 
Fear conditioning test measures the ability of mice to associate aversive experience with environmental cues ${ }^{190,191}$. The overall procedure of the test is based on an unconditioned stimulus (US) followed by a conditioned stimulus (CS) that evoke a conditional response (CR). US can be the spatial cues, odor or audio stimulus. Conditioned stimulus is an aversive stimulus which is generally a foot shock. Conditional response is the freezing behavior, which is expressed as no movement except respiration. After conditioning by applying US followed by CS for several times, the test phase begins. To detect the conditioned fear memory, the mice are exposed only to US and the reaction of mice as the duration of freezing response is measured ${ }^{192}$. There are 2 types of fear conditioning; delayed fear conditioning and trace fear conditioning. In the delayed fear conditioning, US is immediately followed by CS while there is delay between two stimuli in trace fear conditioning. Trace conditioning is a more challenging task for mice. They have to associate the US and the CS. Therefore it requires more repetition during conditioning ${ }^{193}$. Unlike the delayed conditioning, which is hippocampus independent, the trace conditioning requires hippocampal function, suggesting that the absence of contiguity is compensated by the hippocampal activity ${ }^{194,195}$. It was shown that mature miR-132 levels increase 30 min after the trace fear conditioning and then returns back to normal levels within 2 hours. The freezing behavior was impaired when the hippocampal miR-132 levels were knocked-down by infusion of lentivirus carrying anti-miR-132 hairpin RNA into the third ventricle. This finding suggests that the miR-132 function is important to overcome the absence of stimulus contiguity ${ }^{134}$.

\subsubsection{2. miR-212/132 in circadian rhythm}

In all organisms the homeostasis is dependent on cyclic events that influence the regulation of biochemical pathways. The day and light cycle is the most prominent periodical environmental cue, which influences the organismal behavior and metabolism of all multicellular organisms. The circadian rhythm is the observed changes in an organism, which are dependent on external factors, in particular the 'light'. Circadian clock is the molecular mechanisms that keep the inner time ${ }^{196}$.

SCN is a structure in the mammalian brain that harbors the master circadian pacemaker of the body. MiR-132 shows rhythmic expression in SCN but not in cortex. The pre-miR-132 and mature miR-132 induction is light dependent and phase restricted. The brief light exposure 
of the mice in the middle of the respective day did not enhance the expression, whereas the induction could be mediated only during the early and late night phases. The induction is ERK/MAPK pathway dependent. The infusion of miR-132 antagomir into the lateral ventricles potentiated the light induced phase shifting by 2 -folds. The mice that were kept in complete darkness for 8-10 days were briefly exposed to a certain bright level of light. In the mice of control group, such brief exposure cause $65 \pm 8.4 \mathrm{~min}$ phase delay. However in miR-132 antagomir infused mice the delay was $55 \pm 9.7 \mathrm{~min}$ which is significantly lower compared with the control group ${ }^{123}$. On the other hand, overexpression of miR-132 in forebrain neurons, including the $\mathrm{SCN}$, led to a $45 \pm 5.8$ min phase delay while the delay in the control group was $98 \pm 7.7 \mathrm{~min}^{132}$. These results suggest that miR-132 functions as a negative regulator of circadian clock resetting.

Perl and Per2, are period genes which involve in the regulation of the feedback loops of circadian rhythm genes ${ }^{196}$. MiR-132 expression increases Perl transactivation in vitro, while miR-132 antagomir treatment reduces its abundance in vivo upon brief light induction which indicates that miR-132 positively modulates the Perl transactivation ${ }^{123}$. On the other hand in vivo overexpression of miR-132 in the mouse SCN leads to a decrease in the amplitude of Perl levels' rhythmic fluctuations ${ }^{132}$.

Regulatory factor X4 (RFX4), Jumonji, AT-rich interactive domain 1A (JARID1A), methyl Cpg-binding protein 2 (MecP2), p300, B-cell translocation gene 2 (BTG2), polyadenylate-binding protein-interacting protein 2 (PAIP2A) were shown to be targets of miR132 in the mouse SCN. RFX4 is a helix-loop-helix transcription factor, which is highly expressed in the SCN and the testis. Its expression is inducible by light exposure during the night phase $^{197,198}$. RFX4 was shown to be a target of miR-132 in vitro. As expected, the ectopic expression of miR-132 in HEK293 cells leads to decreased RFX4 protein levels ${ }^{123}$.

MecP2 binds to the methylated $\mathrm{CpG}$ dinucleotides that are mostly located at actively transcribed loci in the genome. MecP2 was first defined as a gene expression repressor ${ }^{199}$. However gain- and loss-of-function studies showed that $80 \%$ of the genes whose expression are associated with MecP2 are actually activated by MecP2. This activation requires interaction of MecP2 with $\mathrm{CREB}^{200}$. It also functions during alternative RNA splicing ${ }^{201}$. The level of MecP2 is downregulated in miR-132 overexpressing SCN. Oppositely the miR-132 antagomir treatment 
augmented its levels. MecP2 binds to the 5' regulatory regions of Perl and Per2 gene and their transactivation is positively modulated by MecP2 in a CREB dependent manner ${ }^{132}$. Therefore the downregulated MecP2 levels in miR-132 overexpressing SCN may explain the observed reduction of Per1 levels in these mice.

P300 functions in chromatin remodeling via its intrinsic acetyltransferase activity ${ }^{202}$. It has been shown to be a molecular target of miR-132 in Kaposi's sarcoma-associated herpesvirus (KSHV) and human cytomegalovirus (HCMV) infected monocytes ${ }^{144}$. Reduction and upregulation of p300 levels were observed in the SCN of miR-132 overexpressing transgenic mice and miR-132 antagomir treated mice respectively.

$\mathrm{H} 3 \mathrm{~K} 4 \mathrm{Me} 3$ and $\mathrm{H} 3 \mathrm{~K} 4 \mathrm{Me} 2$ are the histone codes for the active transcription sites in the genome $^{203}$. Removal of methyl groups from lysine 4 residue of histone 3 by JARID1A suppresses the gene expression ${ }^{204}$. The increased abundance of $\mathrm{H} 3 \mathrm{~K} 4 \mathrm{Me} 2$ within the SCN of miR-132 overexpresing transgenic mice might be due to the downregulated levels of JARID1 $^{132}$.

BTG2 interacts with POP2/CAF1, which is subunit of deadenylase complex CCR4/POP2. This interaction increases the rate of POP2/CAF1 mediated polyA shortening, which is an important determinant of mRNA turnover ${ }^{205}$. Overexpression of miR-132 attenuates the BTG2 levels ${ }^{132}$. On the other hand, another miR-132 target PAIP2A is a suppressor of translation, which inhibits the interaction between 5'cap binding EIF4G and polyadenylate binding protein (PABP). The circularization of mRNA, which is an important step to mediate the efficient translation, is prevented by PAIP2A function ${ }^{206,207}$. PAIP2A levels were upregulated in the SCN of miR-132 overexpressing mice although the in vitro data suggested that miR-132 targets PAIP2A. Nevertheless overexpressing BTG2 and PAIP2A in vitro increases the turnover of Perl and Per2. Therefore the altered levels of BTG2 and PAIP2A in miR-132 overexpressing mice might be associated with dampened Perl levels ${ }^{132}$.

\subsubsection{3. $\mathrm{miR}-212 / 132$ in drug addiction}

Substance dependence, commonly known as drug addiction, is a compulsive need by a person to function normally. The substance abuse is a rewarding behavior which reinforces them 
to be repeated ${ }^{208}$. The dopamine release from the nucleus accumbens (NA), which is located at ventral striatum, is crucial for self-stimulation, feeding, sexual activity and exercise mediated reinforcements and addiction to morphine, amphetamine, cocaine, ethanol, nicotine and 9 tetrahydro-cannabinol ${ }^{192}$. The dopaminergic pathway in the rat brain originates from A10 ventral tegmental cell bodies and terminate in the NA, olfactory tubercles and medial frontal cortex ${ }^{209}$.

Rett syndrome is an X-linked neurodevelopmental disorder which is the most common reason of mental retardation among females ${ }^{210}$. The patients suffer from developmental stagnation, difficulty in speech, incessant stereotypies, social incompatibility, loss of motor skills, deficit in cognitive functions, anxiety and seizures ${ }^{211}$. MecP2 dysfunction, which involves loss-of-function and overexpression, was determined as molecular basis of Rett syndrome ${ }^{212}$. $\mathrm{MecP} 2$ is an important factor for neuronal activity dependent gene regulations, consequently for synaptic plasticity and thus for learning and memory ${ }^{213-215}$. Since drug addiction is considered as a neuroplasticity disorder ${ }^{216,217}$, MecP2 was proposed to have some roles in the mechanisms of reward and reinforcement ${ }^{218}$.

Extended (6 hours) but not restricted (1 hour) self-administration of cocaine increases the $\mathrm{MecP} 2$ levels in dorsal striatum of rat. If the MecP2 is knocked down in the striatum of mice by lenti-sh-MecP2 infection, the motivation of rats to use cocaine is abolished. Blocking miR-212 however, rescues the decreased motivation to cocaine self-administration in lenti-sh-MecP2 rats. Extended cocaine administration increases the miR-212/132 levels in the dorsal striatum, hippocampus and cerebellum of rats ${ }^{122}$. When MecP2 is knocked down in HEK-293 cells the miR-212/132 levels increases. Moreover MecP2 knockdown boosts the miR-212/132 induction upon cocaine exposure in the dorsal striatum ${ }^{219}$.

MecP2 is an experimentally confirmed target of miR-132 in cortical neurons ${ }^{125}$. Forskolin or $\mathrm{KCl}$ treatment, which upregulates miR-132, decreases MecP2 levels. Knockdown of miR-132 prevents this decrease. On the other hand, blocking miR-132 increases BDNF levels. If MecP2 is knocked down, this increase, which is due to inactivation of miR-132, is not observed. MecP2 knockout mouse, a model of Rett syndrome, shows lower levels of miR-132 and BDNF. Therefore miR-132 downregulates MecP2 and BDNF is induced by MecP2. Since BDNF is known to be miR-132 inducer, there seems to be a homeostatic regulatory loop ${ }^{125}$. 
The MecP2 and BDNF regulation in striatum upon extended cocaine exposure is different than their regulation in cortical neurons. BDNF levels increase in dorsal striatum of rats which have extended access to cocaine for 24 hours. Lenti-sh-MecP2 rats show reduced BDNF levels in restricted and extended groups. MiR-212 targets MecP2 in striatum as well. Blocking miR212 decreases BDNF levels in both restricted and extended groups. When BDNF antibody is administered to striatum of rat, decrease in motivation to cocaine consumption is observed. Therefore MecP2 blocks miR-212 and BDNF while miR-212 attenuates BDNF in an indirect way. Homeostatic interaction between MecP2 and miR-212 control the magnitude of BDNF expression in striatum upon cocaine exposure ${ }^{219}$.

MiR-212 was found to be involved in CREB signaling while regulating motivation for cocaine intake. Lentiviral miR-212 overexpression in the dorsal striatum of rat decreased the motivation of cocaine consumption. MiR-212 overexpression potentiates the CREB signaling, which can also be mediated by forskolin treatment. Phosphorylation of CREB and induction of Fos mRNA and protein, is enhanced when miR-212 is overexpressed. This enhancement is dependent on TORC, which is a CREB coactivator. Cyclic adenosine monophosphate (cAMP) protects TORC from degradation by triggering $\mathrm{CBP} / \mathrm{p} 300$ mediated acetylation of TORC ${ }^{220}$. MiR-212 expression increases TORC and acetylated TORC levels. Raf1 is a kinase that activates adenylyl cyclases ${ }^{221}$. It needs to be phosphorylated in order to be activated as well ${ }^{222}$. MiR-212 overexpression increases the levels of phosphorylated Raf1 and Raf1 activation increases TORC, phosphorylated CREB (pCREB) and cAMP levels. Sprouty-related, EVH1 domain containing 1 (SPRED1) is Raf1 repressor ${ }^{223}$. It is targeted by miR-212. The overexpression of miR-212 in dorsal striatum decreases SPRED1 levels. SPRED1 knockdown potentiates pCREB induction by forskolin treatment without altering total CREB (tCREB) levels and increases TORC expression, which phenocopies the miR-212 overexpression. However the expression of the transgenic SPRED1 that lacks 3'UTR attenuates but not blocks the stimulatory effect of miR-212 on pCREB levels ${ }^{122}$. Therefore at least in part miR-212 activates TORC-CREB pathway through activation of Raf1 by repressing SPRED1. 


\subsection{3. $\quad \operatorname{miR}-212 / 132$ in neurological disorders}

Misregulation of miR-212/132 expression was observed in various neurological disorders. Analysis of postmortem tissues obtained from schizophrenic patients showed downregulation of miR-212 while patients suffering from bipolar disorder had upregulated miR$212 / 132$ levels $^{224,225}$.

A severe form of neural tube defect, anencephaly, is the result of unclosed neural tube in the cranial region. The fetus, which does not develop telencephalon, cannot survive ${ }^{226}$. The brain samples obtained from the fetuses with anencephaly shows downregulated miR-212 levels ${ }^{227}$.

Differential expression of miRNAs was also shown in patients with certain neurodegenerative diseases, such as Huntington's, Parkinson's and Alzheimer's diseases. Both in the mouse model of Huntington's disease and patients' postmortem brain samples, miR-132 were shown to be downregulated ${ }^{228,229}$. The mouse Parkinson's model $\alpha$-synuclein(A30P) transgenic mice also shows lower levels of miR-212 and miR-132 in their brainstem ${ }^{230}$. MiR-212 was shown to be downregulated in the white matter of Alzheimer's disease patients ${ }^{231}$. Hippocampus and medial frontal gyrus, which are the regions primarily affected in Alzheimer's disease had lower miR-212 levels in Alzheimer's patients compared to healthy subjects ${ }^{232}$. Additionally the change in miR-212 levels seems like in correlation with the appearance of neurofibrillary tangles, a marker for the late stage Alzheimer's disease ${ }^{231}$.

Progressive supranuclear palsy (PSP) is a form of taupathy, which is caused by the hyperphosphorylation and aberrant accumulation of microtubule associated protein tau in neurons. Tau protein has 6 isoforms generated by alternative splicing of exon 2, 3 and 10 . Inclusion of exon 10 leads to production of three or four (3R-tau or 4R-tau) microtubule binding repeats $^{233}$. The imbalance of $4 \mathrm{R}$-tau to $3 \mathrm{R}$-tau (4R:3R-tau) is observed in the taupathies ${ }^{234}$. Altered 4R:3R-tau ratio and downregulated miR-132 was observed in the prefrontal and temporal lobes of PSP patients. Polypyrimidine tract-binding protein 2 (PTBP2) that has role in exon inclusion in alternative splicing, is targeted by miR-132. It is upregulated in prefrontal and temporal lobes of PSP patients. The knockdown of PTBP2 alters 4R:3R-tau ratio. Therefore the alteration in tau production might be linked to PTBP2 upregulation which might be due to the miR-132 downregulation in PSP patients ${ }^{145}$. 
It was shown that evoking status epilepticus in mice by intra-amygdalar kainic acid application upregulates miR-132 levels in the CA3 region of the hippocampus. Interestingly in the preconditioned mice which are tolerant to damaging insult of epileptic seizures, miR-132 was downregulated compared to intolerant mice. When miR-132 was depleted by antagomir treatment, seizure-induced neuronal death was observed to be attenuated ${ }^{127}$.

Serum levels of BDNF and miR-132 was shown to be different in patients suffering from depression compared to healthy individuals. Therefore, measuring the BDNF and miR-132 levels in the serum was proposed as a diagnostic test for clinical depression.

\subsection{HIPPOCAMPUS}

Hippocampus is part of medial temporal lobe. There are 2 hippocampi located under the cortex and they are responsible for the acquisition and consolidation of different aspects of memory ${ }^{235}$. The term "hippocampus" was first used by the Italian anatomist Arantius in 1564 . Hippocampus is the Greek word for "seahorse" and obviously got this name from its sea-horse like appearance. Hippocampus is also reminiscent of ram's horn. In 1742, Parisian surgeon Rene Jacques Croissant de Garengeot suggested the name "cornu ammonis" or "Ammon's horn" which refers to the Egyptian god Amun Kneph, whose symbol is a ram. Cornu Ammonis (CA) became the three different subdivisions of hippocampus, CA1, CA2 and CA3, which are identified by American neuroanatomist Rafael Lorente de Nó in 1934.

\subsubsection{Hippocampal formation}

Several different cytoarchitectural structures are grossly defined as hippocampus or hippocampal formation. The subdivisions of hippocampus are dentate gyrus (DG), CA1, CA2, CA3, subiculum, presubiculum and parasubiculum. Entorhinal cortex (EC) is part of parahippocampal cortex together with perirhinal cortex and postrhinal cortex ${ }^{236}$. Hippocampus and parahippocampal gyrus make up the medial temporal lobe ${ }^{237}$. If the DG is considered as the proximal site, the order of structures down to the distal site is DG, CA3, CA2, CA1, subiculum, presubiculum, parasubiculum and EC.

DG is $\mathrm{U}$ or V shaped structure with three layers. The layer between the granule cell layer and CA1 is called the suprapyramidal blade. The CA1 and suprapyramidal blade is separated by 
hippocampal fissure. The opposite portion is called the infrapyramidal blade. The region linking two blades is the crest.

The CA1, CA2 and CA3 differ in terms of their afferents and efferents. Besides, the size of the pyramidal cells in CA2 and CA3 is greater than CA1. The principal cellular layer is pyramidal cell layer. The layer that contains the basal dendrites of pyramidal cells is called the stratum oriens (so). In the CA3 region, the area which is occupied by mossy fibers is called stratum lucidum (sl). Stratum radiatum (sr) is located superficial to sl in CA3 and pyramidal cell layer in CA2 and CA1. Stratum lacunosum-moleculare (sl-m) is the most superficial layer of the hippocampus.

Subiculum, presubiculum and parasubiculum are altogether called subicular complex. The border between the CA1 and subiculum is where the Schaffer collateral projections from the CA3 region end. It is the point that condensed pyramidal cell layer of CA1 broadens into thicker layer of the subiculum. The presubiculum lies adjacent to subiculum. It is characterized by the external cellular layer composed of small and densely packed pyramidal cells. Parasubiculum has wedge-shaped layer II with pyramidal cells that are larger and less compact than the cells in presubiculum. EC is multilamellar (layer I to IV). Layer I is cell-poor but rich in fibers with transverse orientation. Layer II contains stellate cells and clusters of pyramidal cells. Layer III contains predominantly pyramidal cells. Layer IV is a cell-free layer especially at caudal levels of EC. Cells from layer III and V invade the layer IV that gives patchy appearance in the rest of the EC. Layer $\mathrm{Va}$ is composed of densely packed large pyramidal neurons. The packaging density is lower at deeper parts and the cells are smaller which makes up layer $\mathrm{Vb}$. Heterogeneous cell size and shape is found at layer VI. The density of cells decrease as the subcortical white matter is approached ${ }^{235}$.

\subsubsection{Hippocampal Networks}

The feature of hippocampus which makes it unique among the brain structures is that the information flow through the hippocampal subdivisions is unidirectional. Highly convergent and divergent design of its connections enables it to receive and further processes the multimodal sensory information from the variety of neocortical connections. 


\subsubsection{Connections of DG}

DG receives input dominantly from the EC via perforant pathway. The projections mainly arise from the layer II of EC while some afferents are from layer $\mathrm{V}$ and $\mathrm{VI}^{238}$. The perforant path fibers terminate only in the molecular layer of $\mathrm{DG}^{239}$. Projections from medial septal nuclei and diagonal band of Broca, which are found at basal forebrain, project to polymorphic layer of DG via fimbria, dorsal fornix, supracallosal stria and ventral route through and around amygdaloid complex ${ }^{240}$. Projections from supramammillary area in the hypothalamus terminate mainly in the narrow zone of the molecular layer where dendrites of granule cell layer are found ${ }^{241}$. DG receives several different afferents from brain stem. Locus coeruleus projects noradrenergic fibers to polymorphic layer of DG and sl of $\mathrm{CA} 3^{242}$. Some minor dopaminergic projections arise from ventral tegmental area ${ }^{243}$. Serotonergic projections from medial and dorsal raphe nuclei terminate in the polymorphic layer of $\mathrm{DG}^{244}$.

DG projects exclusively to CA3 region via axons called mossy fibers. The projections terminate just at the border of $\mathrm{CA} 3$ and $\mathrm{CA} 2$. Not getting input from mossy fibers is distinguishing feature of CA2 from $\mathrm{CA} 3^{245}$. Each granule cell has ability to activate $15 \mathrm{CA} 3$ pyramidal neurons. Each CA3 pyramidal neurons receive input from 72 granule cells ${ }^{246}$.

\subsubsection{Connections of CA region}

The perforant path that originated from layer II of EC terminates both at DG and CA3/CA2 region ${ }^{247}$. Therefore it can be speculated that the information carried from EC to DG and CA3/CA2 are the same. The projections from EC to CA1 arise in layer III. The connection between CA1 and EC is reciprocal. CA1 sends its axon from where it receives. Proximal CA1 cells project to medial EC while distal CA1 neurons project to lateral $\mathrm{EC}^{248}$.

There is no known connection between $\mathrm{CA} 3 / \mathrm{CA} 2$ regions to neocortex. $\mathrm{CA} 3$ receives input from basal nucleus of amygdaloid complex. CA1 projects to amygdaloid complex and has reciprocal connection with the perirhinal cortex as well. Moreover CA1 sends its axons to postrhinal and retrosplenial area ${ }^{249,250}$.

The major forebrain region where CA3 gets input is septum. The projections arise from medial septal nucleus and the nucleus of the diagonal band of Broca like the projections reach to 
DG. CA3 sends its axons to lateral septal nucleus via fimbria and precommissural fornix. CA1 also project to lateral septal nucleus. However CA1 projection to lateral septal nucleus is ipsilateral while CA3 is bilateral ${ }^{251}$.

The known hypothalamic connection with CA region is between CA2 and supramammillary area and the tuberomammillary area at posterior hypothalamus. The projection is from hypothalamus to $\mathrm{CA} 22^{241}$. The nucleus reuniens, located in the thalamus, give rise to projections to sl-m of CA1, where the fibers overlap with the axons from EC. The projections terminate on both principal cells and GABAergic interneurons ${ }^{252}$.

Hippocampus receives noradrenergic and serotonergic inputs from brain stem. Noradrenergic fibers that arise from locus coeruleus are distributed in sl and sl-m layers. Serotonergic fibers do not form synaptic connections and appear to release the neurotransmitters into extracellular space of CA3 region. CA1 receives more monoaminergic fibers than CA3, which effects $\mathrm{LTP}^{243}$.

Although hippocampus receives many inputs from diverse structure of the brain, the major input is coming from the hippocampus itself. The CA3 projection to CA3 and CA2 is called associational connections. The axons that arise from CA3 and reaches to CA1 field are Schaffer collaterals. The CA3 neurons, which lay close to DG, project more heavily to septal levels of CA1. CA3 neurons located closer to CA1 project to temporal levels of CA1. The CA3 neurons also distribute its fibers to the same regions of contralateral hippocampus as within ipsilateral hippocampus ${ }^{253,254}$. The projections that reach to the contralateral hippocampus are called commissural projections ${ }^{255}$. Interestingly commissural projections are less abundant in monkey than rat and mice, and almost absent in humans.

\subsubsection{Connections of Subiculum}

Subiculum is the primary source of efferent connections from the hippocampal formation. Therefore it can be considered as the last step in the information processing through the hippocampus. Subiculum projects to layer I and II of the presubiculum and parasubiculum. It is connected reciprocally to the EC. Fibers that arise from layer III and II of EC terminate in the 
subiculum. The projections from subiculum reaches to every region of EC but most prominently at layer $\mathrm{V}^{256}$.

The subiculum projects strongly to layer II and III of retrosplenial cortex and perirhinal $\operatorname{cortex}^{257}$. It receives projections from the amygdaloid complex and projects back to accessory basal nucleus. Ventral subiculum projects strongly to the bed nucleus of the stria terminalis and moderately to claustrum and endopiriform nucleus ${ }^{249}$.

The main projection of subiculum to the basal forebrain is to lateral septal nuclei and nucleus accumbens. The connection between subiculum and the nucleus accumbens is unidirectional however subiculum receives some weak cholinergic fibers from septal nucleus.

Subiculum is connected to mammillary nucleus in hypothalamus. It projects to and receives fibers from medial and lateral mammillary nuclei. The thalamic inputs to subiculum are like thalamic inputs that CA1 receives. The inputs mainly arise at the nucleus reuniens, the paraventricular nucleus and the parataenial nucleus. Subicular input to thalamus terminate at nucleus reuniens, nucleus interanteromedialis, paraventricular nucleus and nucleus gelatinosus.

\subsubsection{Connections of presubiculum and parasubiculum}

Presubicular fibers only project to medial EC. The fibers terminate mostly at layer III and at small extent at layer I. Parasubiculum innervates at layer II of the medial and lateral EC. The presubiculum projects to layer I and II of parasubiculum. Parasubiculum project back to layer I and III of the presubiculum.

The most dominant neocortical input to presubiculum comes from retrosplenial cortex. Fibers from layer V of retrosplenial cortex terminates in layer I, III and V of the presubiculum. Projections back to retrosplenial cortex arise from layer $\mathrm{V}$ of presubiculum and terminate at layer I and II. Layer V of the visual area $18 \mathrm{~b}$ innervate to layer I and III of presubiculum ${ }^{257}$.

Presubiculum and parasubiculum reciprocally connect with anterior thalamic nuclear complex. Thalamic projections terminate at layer I, III and IV of presubiculum. The presubicular projections to anterior thalamic nuclear complex arise from layer IV. 
Layer II of presubiculum also receives input from medial septal nucleus and diagonal band of Broca. Serotonergic projections from dorsal and ventral raphe nuclei terminate at layer I and noradrenergic locus coerulus at plexiform layer of presubiculum. Hypothalamic connections of presubiculum at medial and lateral mammillary nuclei are reciprocal ${ }^{258}$.

\subsubsection{Connections of entorhinal cortex}

EC is the main structure where the processed sensory information coming from hippocampus enters and relays back to the neocortex. It is the beginning and end point of the loop of information processing. Father of modern neuroscience Ramon y Cajal says that whatever the rest of the hippocampal formation is doing depends on what the EC has done.

Perforant path that arise from lateral and medial EC projects to DG, CA region and subiculum. Projections from layer II terminates at DG and CA3 and layer III neurons project to CA1 and subiculum. DG and CA3 do not project back to EC. The return projections from CA1 and the subiculum terminates at layer I, V and $\mathrm{VI}^{248,259}$.

EC receives substantial amount of "raw material" to be processed in the hippocampal formation from the extrinsic connections. The percentages of inputs the EC receives from different cortical regions are summarized in table $2^{260}$.

Table 2: The percentages of projections that $\mathrm{EC}$ receives from different associational cortices.

\begin{tabular}{|l|c|c|}
\hline & \% of total input to lateral EC & \% of total input to medial EC \\
\hline Piriform cortex & 34 & 31 \\
\hline Temporal cortex & 26 & 21 \\
\hline Frontal cortex & 11 & 10 \\
\hline Insular cortex & 21 & 6 \\
\hline Cingulate cortex & 3 & 11 \\
\hline Parietal cortex & 3 & 9 \\
\hline Occipital cortex & 2 & 12 \\
\hline
\end{tabular}

The neocortical inputs that terminate in layer I and II of the EC yield information to neurons which then project to DG, CA region and subiculum. The layer IV and VI neurons, 
which are innervated by neocortical regions as well, receive information from other hippocampal areas and give rise to feedback projections to certain neocortical regions.

Olfactory bulb, anterior olfactory nucleus and piriform cortex project strongly to layer I and II of EC. Prominent postrhinal and perirhinal projections terminate in the medial EC and lateral EC respectively. Perirhinal and postrhinal cortex are convergence area of variety of sensory cortices. Perirhinal cortex is composed of area 35 and 36. Area 36 receives most of the inputs from ventral temporal associational area, postrhinal cortex and EC. Area 35 receives projections from piriform, entorhinal and insular cortices. Perirhinal projections to EC arise mostly from area $35^{261}$. Agranular insular cortex, infralimbic, prelimbic, anterior cingulate cortices and the retrosplenial cortex give rise to projections to $\mathrm{EC}$ as well ${ }^{262}$.

The return projections to olfactory areas originate from layers II, III and Va. The projections from EC to infralimbic, prelimbic, orbitofrontal, agranular insular, retrosplenial cortex, perirhinal and postrhinal cortices originate from layer $\mathrm{Va}$.

Among the subcortical connections to EC, lateral amygdaloid nucleus fibers terminate at layer I, II and III. Projections from basal nucleus arrive to III and V and fibers from cortical nucleus and periamygdaloid cortex innervate to layer I and II. Feedback projections from EC to amygdala arise from layer $\mathrm{V}^{250}$. EC neurons at layer $\mathrm{V}$ also projects to nucleus accumbens in striatum.

EC receives cholinergic inputs from septum at layer II and projects back from neurons at layer Va and II. EC also receives inputs from supramammillary nucleus and tuberomamillary nucleus distributed diffusely throughout the EC. Thalamic inputs to EC originate in nucleus reuniens, nucleus centralis medialis, rhomboid, paraventricular and parataenial nuclei. Nucleus reuniens fibers innervate layer I, II and III $^{263}$.

EC receives dopaminergic input from ventral tegmental area, serotonergic input from the dorsal and central raphe nuclei and noradrenergic inputs from locus coeruleus ${ }^{258}$. 


\subsubsection{Spatial representation system}

In 1957, to relieve seizures of antiepileptic drug-resistant patient H.M., medial aspects of temporal lobes (hippocampus, amygdala, collateral sulcus, perirhinal cortex, EC and mammillary nucleus) were resected. Seizures decreased and personality, early memories, general intelligence and technical skills of H.M. were intact however anterograde amnesia was reported. It was the first time that involvement of hippocampus in memory formation was expressed ${ }^{264}$.

Long-term memory is composed of declarative and non-declarative memory. Declarative memory is further divided into semantic and episodic memories. Semantic memory is the memory of facts about the living things, objects, places and events without temporal or contextual references like "water is liquid" or "the capital city of Germany is Berlin". On the other hand, episodic memory deals with the first-person experiences with space and time components. For example "I visited the Louvre Museum in Paris 2 years ago" can be an example for episodic memory. It is self-referenced (egocentric). Non-declarative memory is the memory without awareness. Recognizing a face without denying that the face is familiar, learning complex motor actions like riding bike or learning a conditioned response like Pavlov's dogs salivate as they hear the ring of the bell are examples for non-declarative memory. Declarative memory is highly dependent on hippocampal-entorhinal system while non-declarative is not ${ }^{265}$.

Water maze assay is a behavioral assay that is used to study the spatial learning ability of mice and rats. The task is that the animal should escape from water onto a hidden platform. The animal navigates itself onto the platform by using the spatial cues around. If the animal has intact spatial memory, it shows decreasing escape latency in each repetition ${ }^{266}$. When the hippocampus, subiculum or both of the structures are lesioned, the rats do not swim to the target quadrant while sham controls $\mathrm{do}^{267}$. Therefore hippocampal formation is required for proper spatial memory.

The patient E.P., who had bilateral damage to their hippocampus and related medial temporal lobe structures, could not remember recently acquired spatial information. However he could recall the spatial features of his neighborhood that he left 50 years ago. Therefore hippocampus is important for the acquisition and consolidation of spatial memory for certain amount of time ${ }^{268}$. 
It was 1971 when it was realized that group of neurons in the hippocampus fires depending on the position of the rat in a space and the neighboring cells fire when the animal changes its position ${ }^{269}$. Those neurons are pyramidal neurons and called place cells ${ }^{270}$. Almost all pyramidal cells in CA region are place cells ${ }^{271-273}$. At presubiculum and parasubiculum and the upstream of the "place cells", mainly in the layer II and III of the medial EC, there are "grid cells"274-276. Those cells have firing fields that covers the space in triangular pattern" ${ }^{277,278}$. Presubiculum, thalamus and deep layer of EC contain "head direction cells" which fire as the animal turns its head to another direction ${ }^{275,279}$. Another kind of neurons which constitute $>10 \%$ of local neuron population of EC and parasubiculum, fire when the animal is close to the borders of the space. Those cells are called "border cells" 280 .

Place cells inform the location of the subject. Grid cells create a neural representation of the geometry of the environment. Head direction cells provide information about the orientation of the body in space and border cells contribute by determining the size of the discoverable space. Presence of those cells in EC-hippocampal system, lesion studies and investigation of patients with medial temporal lobe damage, suggest that hippocampal formation has important functions in spatial processing. 


\subsection{Scope of the thesis}

The emerging understanding of functions of miRNAs presents them as indispensable molecular factors in the regulation of dynamic neuronal processes. MiR-132, which was first defined as a brain specific miRNA ${ }^{111}$, and miR-212 has been studied for more than 8 years. However their functions in cognition, synaptic plasticity and the regulation of relevant molecular pathways have not been fully understood.

The main goal of this study is to elucidate the in vivo functional roles of miR-212/132 family with particular focus on neuronal systems by utilizing the miR-212/132 loss-of-function mutant mouse model (miR-212/132 $2^{-/}$). Towards this goal, different experimental approaches had been used in this study:

1. The spatial expression pattern of miR-212/132 gene in adult mouse brain and expression levels of miR-212/132 in peripheral mouse organs was studied.

2. A large battery of behavioral assays were performed in order to demonstrate the role of miR-212/132 in the regulation of cognitive behaviors in mouse

3. Electrophysiological assays were performed to reveal the involvement of miR-212/132 in the regulation of the synaptic transmission.

4. Molecular function studies were performed to investigate miR-212/132 function in the regulation of prospective signaling pathways. 


\section{MATERIALS AND METHODS}

\subsection{Methods}

\subsubsection{Ethics Statement}

All animal procedures were performed as approved by the institutional animal care and use committee of Max-Planck Institute for Biophysical Chemistry and Max-Planck Institute of Experimental Medicine with permission (33.42502/02-07.05 A) of the Bezirksregierung Braunschweig (Local Animal Care and Use Committee) in accordance with the German Animal Protection Law.

\subsubsection{Behavioral tests}

The behavioral tests were performed by Dr. Konstantin Radyushkin in the research group of Prof. Dr. Dr. Hannelore Ehrenreich in Max Planck Institute for Experimental Medicine. Agematched male wild-type $(+/+, \mathrm{n}=23)$ and miR-212/132 $2^{-/-}$mutant $(-/-, \mathrm{n}=22)$ littermates were used in behavioral tests. Mice were housed at 4-5 animals per cage in a room with 12 hrs light-dark cycle and ad libitum access to food/water. Mice were 10-11 weeks old at the beginning of the assays. Inter-test interval was at least 1-2 days. The tests were performed in the following order: Elevated plus maze, open field, rotarod, social interaction, pre-pulse inhibition, 8-arm maze test, Morris water maze, cued and contextual fear conditioning. The order of testing was designed according to increasing invasiveness to minimize a possible influence of experimental history ${ }^{281}$. All mice were used for all behavioral tests. The experimenter was unaware of the genotype of mice.

\subsubsection{Elevated plus maze}

The test of anxiety was performed as described previously ${ }^{282}$. The behavior of the mice, which was placed in the central platform, recorded for $5 \mathrm{~min}$ to measure the time spent in open and closed arms, total distance traveled, number of arm visits and the velocity. The ratio of time spent in open arm to closed arms was used to interpret the anxiety levels of the mice. 


\subsubsection{Hole board}

The mice were put in the middle of hole board chamber with equally spaced 12 holes. The distance traveled and the number of head dips into holes were assessed for $3 \mathrm{~min}$.

\subsubsection{Open field}

The attitude of mice in Perspex arena was observed to examine the spontaneous activity in the open field. Mice were allowed to explore the arena for $7 \mathrm{~min}$. Then the behavior of mice was recorded to calculate the velocity, distance traveled, and time spent in central, intermediate or peripheral zones of the open field.

\subsubsection{Rotarod}

The rotarod test is performed to check whether the motor function, balance and coordination of mice are altered. Mice were placed individually on the drum, which was accelerated from 4 to $400 \mathrm{rpm}$ in $5 \mathrm{~min}$. The latency of falling off the drum was measured.

\subsubsection{Social interaction}

The social interaction test was performed as described previously ${ }^{283}$. The social testing arena was a rectangular, 3-chambered box with access into each chamber. The mice were let for habituation in the middle chamber for $5 \mathrm{~min}$. An unfamiliar C57BL/6NCrl male mouse (stranger 1) was enclosed in a small rectangular wire cage in one of the side chambers. The location of the stranger 1 was changed from the left chamber to the right chamber or vice versa between trials. An identical empty wire cage was placed in the opposite chamber. After habituation period mouse was allowed to explore all chambers of the test arena for a $10 \mathrm{~min} / \mathrm{session}$. The number of entries into each chamber and the time spent in each chamber were recorded. At the end of the first $10 \mathrm{~min}$-trial, each mouse was tested in a second $10 \mathrm{~min}$-session to quantify social preference for a new stranger (stranger 2). The stranger 2 was placed into the empty wire cage. The test mouse preference to spend time with the familiarized stranger 1 versus stranger 2 was measured by recording the amount of time the test mouse was present in each chamber and the number of 
transitions between the chambers. The 'sociability index' and a 'social memory index' were calculated according to the following formulas:

Social index $=\left(\left(\right.\right.$ Time $_{\text {stranger } 1} /\left(\right.$ Time $_{\text {stranger } 1}+$ Time $\left.\left.\left._{\text {empty }}\right)\right) \times 100\right)-50$.

Memory index $=\left(\left(\right.\right.$ Time $_{\text {stranger } 2} /\left(\right.$ Time $_{\text {stranger } 2}+$ Time $\left.\left.\left._{\text {stranger } 1}\right)\right) \times 100\right)-50$.

\subsubsection{Pre-pulse inhibition test:}

Prepulse inhibition test is performed to assess sensorimotor gating. The mice were put in a small cage which restricts the movement of mice and can record the vertical movements of the floor. Acoustic stimuli delivered from a loudspeaker evoked a startle response and consequently created a transient force which is due to the movement of the platform. The force was recorded. To test pre-pulse inhibition, the $120 \mathrm{~dB} / 40 \mathrm{~ms}$ startle pulse was applied either alone or following a pre-pulse stimulus of $70 \mathrm{db}, 75 \mathrm{db}$, or $80 \mathrm{~dB}$ intensity and $20 \mathrm{~ms}$ duration. Background white noise was applied between each pre-pulse and pulse stimulus for $100 \mathrm{~ms}$. The trials were repeated in an interval of 8 to 22 seconds. The startle response was expressed as the difference between the maximum force detected during the recording time and the force measured immediately after the stimulus application. Pre-pulse inhibition was calculated as the percentage of the startle response using the following formula:

$\%$ pre-pulse inhibition $=100-[($ startle amplitude after pre-pulse and pulse $) /($ startle amplitude after pulse only) $x$ 100]

\subsubsection{8-Arm radial maze test:}

The mice which are deprived of water were trained to explore and consume the liquid reward in the magazine located at the end of each arm of 8-arm radial maze. Following the training sessions, the mice were located to the central platform of the maze and allowed to consume the liquid rewards on each arm for $15 \mathrm{~min}$. Each trial was stopped either when $15 \mathrm{~min}$ is over or all rewards were consumed. The working memory was expressed as the errors done while choosing an arm to visit and latency to consume all the rewards. Errors were defined as revisit to arms. 


\subsubsection{Morris water maze:}

The spatial learning and memory abilities of mice were investigated in Morris water maze. The mice were trained to find the visible platform located at different locations of the maze without extra-maze cues for 2 days. The mice were trained to find the hidden platform, which is located at the center of one of the 4 quadrants of the pool. The mice were expected to navigate by using the extra maze cues that were placed on the walls around the pool. 4 trials were performed each day. The mice were put to one of the 4 quadrants randomly. If the mice could not find the platform in $90 \mathrm{sec}$, they were guided to the platform. Animals stayed on the platform for $20 \mathrm{sec}$. The training continued for 8 days. The escape latency, swim speed, path length, and trajectory of swimming were recorded for each mouse. Following the hidden platform training, probe trial was performed. The platform was removed and mice were allowed to swim for $90 \mathrm{sec}$. The time spent in each quadrant and the number of times that the mice crossed the point where the hidden platform used was recorded.

\subsubsection{Cued and contextual fear conditioning:}

The fear-conditioning test was performed as described earlier ${ }^{284}$. The mice were exposed to the context for $2 \mathrm{~min}$ to assess the baseline activity. Then conditioned stimulus (CS), which is $5 \mathrm{kHz}$, $85 \mathrm{~dB}$ tone were delivered for $5 \mathrm{sec} .0 .4 \mathrm{~mA}$ foot shock for $2 \mathrm{sec}$, as unconditioned stimulus (US) was applied immediately after the CS. The same CS-US pairing was repeated $13 \mathrm{sec}$ later. The mice were kept in the same chamber for additional $23 \mathrm{sec} .2$ days later the contextual memory test was performed by exposing the mice to the same context. The mice were monitored for $2 \mathrm{~min}$ to assess the freezing reflex. $52 \mathrm{hrs}$ after the training, the mice were transferred into new chamber to test the cued memory. Baseline activity was recorded for the first $2 \mathrm{~min}$. Then the tone was presented and the mice were monitored 2 more minutes to assess the freezing behavior of mice upon the cue. Freezing behavior is defined as the lack of any movement except respiratory movement.

\subsubsection{Electrophysiological analyses}

\subsubsection{Slice preparation}


Electrophysiological analyses were performed by Dr. Jeong Seop Rhee in Max Planck Institute for Experimental Medicine. $400 \mu \mathrm{m}$ thick transverse acute hippocampal slices were prepared from 4 or 5-weeks-old wild-type and miR-212/132-/- mutant mice. The recovery of the slices were performed in artificial cerebrospinal fluid (ACSF) bubbled with $95 \% \mathrm{O}_{2}$ and $5 \% \mathrm{CO}_{2}$ for $2 \mathrm{hrs}$ at $30^{\circ} \mathrm{C}$. Then the slices were perfused with ACSF at flow rate of $2 \mathrm{ml} / \mathrm{min}$ and maintained in a submerge chamber at $25-28^{\circ} \mathrm{C}$ throughout all experiments. The epileptiform activity and trysynaptic pathway activation was prevented by surgically cutting DG-CA3 synapses. $50 \mathrm{M}$ picrotoxin $\left(\mathrm{GABA}_{\mathrm{A}}\right.$ receptor antagonist) was used to isolate field excitatory postsynaptic potentials (fEPSC). Schaffer collateral afferents were stimulated by using bipolar stainless steel electrodes. FEPSPs were measured in the stratum radiatum of CA1 with $2 \mathrm{M}$ $\mathrm{NaCl}$-filled glass electrodes (2-3 M $\Omega$ ).

\subsubsection{I-O curve and PPR protocol}

The ratio of $2^{\text {nd }}$ fEPSP slope to the $1^{\text {st }}$ fEPSP slope were taken at interstimulus intervals of $25,50,100,200,500$ and $1000 \mathrm{~ms}$ and three consecutive responses were averaged to obtain paired-pulse ratios (PPRs). For input-output (I-O) curves, fEPSPs were evoked with $0.1 \mathrm{~ms}$ stimuli at $0.033 \mathrm{~Hz}$ and three consecutive responses were averaged. The slopes of fEPSP were plotted against synaptic volleys.

\subsubsection{LTP, LTD and depotentiation protocol}

The basal fEPSP were obtained at $0.033 \mathrm{~Hz}$. The adjusted stimulation intensity evoked fEPSP that was 30\%-35\% of maximal slope. Early-phase long-term potentiation (LTP) was induced by applying one train of high frequency stimulation (HFS; $100 \mathrm{~Hz}, 1 \mathrm{sec}$ ). For induction of late-phase LTP, 3 trains of HFS were applied at intervals of 20 seconds. To induce LTD, stimulation intensity was adjusted to evoke fEPSP that were about $50 \%$ of maximal fEPSP slope and low frequency stimulation (LFS: $1 \mathrm{~Hz}$ for $15 \mathrm{~min}$ ) was applied. FEPSP slopes were expressed relative to normalized baseline and were averaged into 2 min intervals in experiments. Depotentiation was analyzed as described previously ${ }^{285-287}$. Initially LTP was induced by 2 trains of HFS at intervals of 20 seconds; and then depotentiation was induced either by applying LFS 
( $2 \mathrm{~Hz}$ for $10 \mathrm{~min}$ ) $2 \mathrm{~min}$ after the induction of LTP, or by applying LFS ( $1 \mathrm{~Hz}$ for 15 minutes) 40 min after the induction of LTP.

\subsection{4. $\quad \beta$-Galactosidase staining}

Brains dissected from 5 weeks old wild-type and miR-212/132 mutant mice were used in whole-mount staining for $\beta$-Galactosidase activity. After dissection, mouse brains were fixed in fixation solution for $1 \mathrm{hr}$ at $4^{\circ} \mathrm{C}$ and washed three times in PBS before staining in $\beta$ Galactosidase staining solution for $2 \mathrm{hrs}$ at $37^{\circ} \mathrm{C}$. The staining was stopped by washing the samples in PBS and subsequently pictured using SZX-10 microscopes (Olympus).

For $\beta$-Galactosidase staining on brain sections, brains of 5 weeks old wild-type and miR212/132 mutant mice were dissected and fixed in the fixation solution for $1 \mathrm{hr}$ at $4^{\circ} \mathrm{C}$. After fixation, brains were washed in PBS and embedded in tissue freezing medium (Leica Microsystems, 020108926). $50 \mu \mathrm{m}$ thick sections were prepared. Sections were further fixed in $0.2 \%$ glutaraldehyde solution for $10 \mathrm{~min}$ and washed in LacZ buffer at $4{ }^{\circ} \mathrm{C}$. $\beta$-Galactosidase staining was performed in $\beta$-Galactosidase staining solution at $37^{\circ} \mathrm{C}$ for $72 \mathrm{hrs}$. After the completion of the staining, sections were fixed in $4 \%$ paraformaldehyde for $10 \mathrm{~min}$ and washed in PBS. Sections were mounted in Prolong ${ }^{\circledR}$ Gold antifade reagent (Invitrogen, P36935) with DAPI or with moviol and analyzed with BX-40 and SZX-10 microscopes (Olympus). The distinct histological structures on brain sections, including different cortical layers, hippocampal granular layers and locations of amygdalar nuclei have been determined based on the nuclear DAPI staining results.

\subsubsection{RNA Isolation}

\subsubsection{RNA isolation from tissue}

The procedure was performed as described previously with some modifications ${ }^{288} .1 \mathrm{ml}$ of Trizol was used to isolate RNA from $100 \mathrm{mg}$ of tissue. The tissue was homogenized by tissue homogenizer. Before starting the homogenization, the rod of the homogenizer was operated in DEPC-treated $\mathrm{H}_{2} \mathrm{O}$ for cleaning. The excess water in the rod was cleaned by soaking the rod in acidic phenol (AppliChem, A1624,0500). Then the tissue was pushed to the bottom of the falcon 
tube by the tip of the rod and the homogenizer was operated for at least $30 \mathrm{sec}$. Before continuing the homogenization with the next tissue, the rod was cleaned by operating in DEPCtreated $\mathrm{H}_{2} \mathrm{O}$ for 2 times and phenol was applied. The DNA in the homogenate was sheared by flushing the liquid through $23 \mathrm{G}, 26 \mathrm{G}$ and $27 \mathrm{G}$ size needles, 2 times each. The homogenate was kept at RT for 5 min. Chloroform was added in 1:5 (volume:volume) ratio, shook vigorously for $30 \mathrm{sec}$ and centrifuged at $6900 \mathrm{rpm}, 4^{\circ} \mathrm{C}$, for $22 \mathrm{~min}$. The upper liquid phase was transferred into a clean tube. Isopropanol at 1:1 (volume:volume) ratio was added and mixed vigorously. If the amount of RNA is expected to be low (depending on the size of the starting tissue) the mixture was incubated at $-20^{\circ} \mathrm{C}$ minimum for $2 \mathrm{hrs}$. Then the mixture was centrifuged at $6900 \mathrm{rpm}, 4^{\circ} \mathrm{C}$ for $30 \mathrm{~min}$. The supernatant was removed. To obtain highly pure RNA, phenol-chloroform extraction protocol was followed. The pellet was dissolved in $1 \mathrm{ml}$ of TES. $1 \mathrm{ml}$ of water saturated phenol was added. The mixture was vortexed and centrifuged for 5 min at $13000 \mathrm{rpm}$, at RT. The upper phase was removed into new eppendorf tube. 1:1 (volume:volume) ratio of chloroform was added to remove the excess phenol. The mixture was centrifuged for $5 \mathrm{~min}$ at $13000 \mathrm{rpm}$. The upper phase was removed into new clean tube. NaOAc was added to have final concentration of $0.3 \mathrm{M}$. After mixing isopropanol at 1:1 (volume:volume) ratio was added. The mixture was incubated at $-20^{\circ} \mathrm{C}$ for at least $2 \mathrm{hrs}$. The suspension was centrifuged at $13000 \mathrm{rpm}$, RT for $10 \mathrm{~min}$. The supernatant was removed. $1 \mathrm{ml}$ of $75 \% \mathrm{EtOH}$ was added. The centrifugation was repeated and the supernatant was removed. To get rid of the residual EtOH, short spinning was performed. The pellet was dried at $37^{\circ} \mathrm{C}$ by shaking for $2-3 \mathrm{~min}$ and dissolved in appropriate amount of DEPC-treated $\mathrm{H}_{2} \mathrm{O}$. To facilitate the dissolving process, the solution was incubated at $55^{\circ} \mathrm{C}$ for $5 \mathrm{~min}$. The concentration and purity of RNA was determined by using Nanodrop ND1000 Spectrophotometer. The RNA integrity was determined by running $100 \mathrm{ng}-1 \mathrm{~g}$ of RNA in $1 \%$ agarose gel. RNA was stored at $-80^{\circ} \mathrm{C}$.

\subsubsection{RNA isolation from neurons}

The culture medium was aspirated and cells were washed with chilled DEPC-treated 1X HBSS. $1 \mathrm{ml}$ of Trizol was added into a well of 24-well plate. The cells were homogenized by flushing the liquid through $27 \mathrm{G}$ needle for 3 times. 2001 of chloroform (1:5, volume:volume) was added and centrifuged for $20 \mathrm{~min}$ at $13000 \mathrm{rpm}$, RT. The upper phase was transferred into new tube $1: 1$ ratio of isopropanol was added and vortexed. The mixture was kept at $-20^{\circ} \mathrm{C}$ for 
$\mathrm{o} / \mathrm{n}$. Then the RNA was precipitated by centrifuging for $20 \mathrm{~min}$ at $13000 \mathrm{rpm}$ at RT. The pellet was washed once in $1 \mathrm{ml}$ of $75 \% \mathrm{EtOH}$. The pellet was recollected by centrifuging for $10 \mathrm{~min}$ at $13000 \mathrm{rpm}$ at RT. Then it was dissolved in 4501 of DEPC-treated $\mathrm{H}_{2} \mathrm{O} .501$ of $3 \mathrm{M} \mathrm{NaOAc}$ and 5001 of isopropanol was added and vortexed. The solution was kept at $-20^{\circ} \mathrm{C}$ for $\mathrm{o} / \mathrm{n}$. The RNA was precipitated for the second time by centrifugation at $13000 \mathrm{rpm}$ at RT for $20 \mathrm{~min}$. The supernatant was removed and $1 \mathrm{ml}$ of $75 \% \mathrm{EtOH}$ was added. The pellet was recollected by centrifugation for $10 \mathrm{~min}$ at 13000rpm at RT. The washing step was repeated for one more time. The pellet was dried at $37^{\circ} \mathrm{C}$ by shaking for 2-3 min and dissolved in appropriate amount of DEPC-treated $\mathrm{H}_{2} \mathrm{O}$. To facilitate the dissolving process, the solution was incubated at $55^{\circ} \mathrm{C}$ for 5 min. The concentration and purity of RNA was determined by using Nanodrop ND1000 Spectrophotometer. The RNA integrity was determined by running $100 \mathrm{ng}-1$ g of RNA in $1 \%$ agarose gel. RNA was stored at $-80^{\circ} \mathrm{C}$.

\subsection{6. $\quad$ Protein isolation from hippocampus}

2501 of chilled lysis buffer was used to homogenize hippocampus dissected from single lobe of a mouse brain. The hippocampus was homogenized by tissue grinder in lysis buffer. The tubes were incubated on rotating shaker at $4^{\circ} \mathrm{C}$ for $1 \mathrm{hr}$. The undissolved portion was precipitated by centrifugation at $13000 \mathrm{rpm}$ at $4^{\circ} \mathrm{C}$ for $20 \mathrm{~min}$. The supernatant was transferred into a new tube. The samples were aliquoted in appropriate amount of volumes and stored at $-80^{\circ} \mathrm{C}$.

\subsubsection{Protein isolation from neuron cultures}

2001 of chilled lysis buffer was used to lyse hippocampal neurons within 24-well plates. The cells were homogenized by pipetting up and down and collected into eppendorf tubes. The tubes were incubated on rotating shaker at $4{ }^{\circ} \mathrm{C}$ for $30 \mathrm{~min}$. The undissolved portion was precipitated by centrifugation at $13000 \mathrm{rpm}$ at $4^{\circ} \mathrm{C}$ for $20 \mathrm{~min}$. The supernatant was transferred into a new tube. The samples were aliquoted in appropriate amount of volumes and stored at $80^{\circ} \mathrm{C}$. 


\subsubsection{Western Blotting}

The protein samples were mixed with appropriate amount of loading buffer and boiled at $96^{\circ} \mathrm{C}$ for $5 \mathrm{~min}$ and cooled to $4^{\circ} \mathrm{C}$. Equal amount of samples were loaded into each lane of stacking. $50 \mathrm{~V}$ of constant electrical field was applied to the SDS-Page gel till the protein reached to the resolving gel. Then the voltage was increased to $120 \mathrm{~V}$. After the run is complete, the stacking gel was cut out. The resolving gel and blotting membrane was sandwiched between 2 sheets of Whatmann paper. The wet blotting was mediated in transfer buffer for $70 \mathrm{~min}$ at 400 $\mathrm{mA}$ current. The transfer buffer was prevented to be heated by covering the chamber with ice. When the transfer was completed, the membrane was removed and washed in TBST for $10 \mathrm{~min}$. It was blocked in either 5\% skim milk (AppliChem, A0830,0500) or 1\% BSA (Thermo Scientific, 37520) for $1 \mathrm{hr}$ by shaking at RT. Before primary antibody incubation, excess blocking buffer was removed from the membrane by washing it in TBST for $10 \mathrm{~min}$. The primary antibodies that were dissolved in blocking buffer were incubated with the membrane at $4^{\circ} \mathrm{C}$ by shaking for o/n. Then the membrane was washed for 5 times, each 5 min at RT in TBST. Secondary antibody which was dissolved in appropriate blocking buffer was incubated with the membrane for 1-3 hrs at RT by shaking. Unbound secondary antibody was removed by washing in TBST for 5 times, $5 \mathrm{~min}$ each. $5 \mathrm{ml}$ of developing solution was prepared by mixing pico and femto chemiluminescent substrates (Thermo Scientific, 34087, 34094) at appropriate ratios. After the membrane was incubated in developing solution for $5 \mathrm{~min}$ by shaking, the membrane was put into developing cassette. The Cl-Xposure film (Thermo Scientific, 34090) was exposed to the membrane for appropriate amount of time and the film was developed in film developer.

\subsubsection{Hippocampal Neuron Culturing}

The procedure was performed as described previously with some modifications ${ }^{289}$.

\subsubsection{Poly-L-lysine coated plate preparation}

Poly-L-lysine solution was applied into each well of 24 -well plates. The plates were incubated at $37^{\circ} \mathrm{C}$ for at least $1 \mathrm{hr}$. Then the wells were washed with $\mathrm{ddH}_{2} \mathrm{O}$ twice. The plates were stored at $4{ }^{\circ} \mathrm{C}$ with $\mathrm{ddH}_{2} \mathrm{O}$ within the wells. 


\subsubsection{Hippocampus dissection from embryonic brains}

The pregnant mouse bearing embryo at E17.5 or E18.5 stage was sacrificed and the abdomen of the mouse was rinsed thoroughly with ethanol. The skin and the peritoneum were incised to expose the viscera and the uterus. The uterus was cut out of the abdominal cavity and put into chilled $1 \mathrm{X}$ HBSS in $50 \mathrm{ml}$ falcon tube. The uterine horns were transferred into laminar flow hood. The uterus and the embryonic sac were cut to release the embryos. To dissect the brain, the embryos were hold by the neck with a forceps. Tip of a Dumont no. 7 forceps was inserted just above the nose and pushed to the rear of the skull. The skull and the skin were broken by pulling the forceps upward and pushed aside. The brain was pushed out by squeezing the forceps gently. The dissected brain was transferred into chilled 1X HBSS. The lobes were separated along the midline. The telencephalon was separated and the meningitis was peeled off from the inner and outer surface of the cortex. The exposed hippocampus was cut out with 80 $\mathrm{mm}$ micro scissors and transferred into chilled $1 \mathrm{ml}$ of $1 \mathrm{X}$ HBSS in $15 \mathrm{ml}$ falcon tube. The hippocampi were kept on ice till the dissection of at most 8 pairs of hippocampi is complete.

\subsubsection{Trituration of the hippocampal tissue}

$1 \mathrm{ml}$ of Trypsin solution was added and incubated at $37^{\circ} \mathrm{C}$ for $30 \mathrm{~min}$. After the incubation, 2501 of Dnase was added to prevent the clumping of tissue due to the released DNA from the dead cells. The mixture was kept at RT for couple of minutes. To inactivate the trypsin, the hippocampi was washed by adding $8 \mathrm{ml}$ of DMEM/10\% FBS. The hippocampi within the suspension were let to settle to the bottom of the tube. The supernatant was aspirated and the wash step was repeated. The volume was adjusted to $1 \mathrm{ml}$. The hippocampi were disturbed into small pieces by flushing through Pasteur pipette with wide opening for 10 times. Another Pasteur pipette was fire polished to narrow down the opening. The tissue chunks were homogenized into single cell suspension by pipetting the solution up and down through the Pasteur pipette for 10 times. The cell suspension was left untouched for $2 \mathrm{~min}$ in order to let the remained tissue pieces to settle down to the bottom of the tube. The precipitates were removed out of the suspension by a Pasteur pipette. 


\subsubsection{Layering the neurons}

101 of cell suspension was mixed with 901 of Trypan blue. The cells were counted with hematocytometer. The cell suspension were diluted to appropriate concentration with DMEM/10\% FBS. Neurons were layered to have 52600 cells $/ \mathrm{cm}^{2}$ in each well. Plates were incubated at $37^{\circ} \mathrm{C}$ for $2 \mathrm{hrs}$ to let the neurons attach to the surface of the wells. Afterwards the cell medium was replaced with $1 \mathrm{ml}$ of culturing medium.

\subsubsection{Maintenance of neurons}

Hippocampal neuron cultures were incubated in a humidified cell culture incubator with $5 \% \mathrm{CO}_{2}$ at $37^{\circ} \mathrm{C}$. Half of the culture medium was replaced with fresh Neurobasal medium with B27 supplement but without glutamate at day in vitro 4 (DIV4). The purpose in this step is to decrease the glutamate concentration by half. Drug application and protein/RNA isolation were performed on DIV9.

\subsubsection{Drug application}

The volume in each well was checked in order to assess the volume loss during culturing due to the evaporation. The missing part was replaced with Neurobasal medium without any additive. Appropriate amount of forskolin, which was dissolved in DMSO, was diluted in pure Neurobasal medium to the concentration of 110 M. 1001 of the dilution was added into the wells which made the final forskolin concentration $10 \quad 1$. Control hippocampal neurons were treated with DMSO only. When the incubation period was over the culture medium was aspirated. The cells were washed with $1 \mathrm{ml}$ of chilled $1 \mathrm{X}$ HBSS. Then the RNA or protein isolation protocols were followed.

\subsubsection{Real Time PCR Assays}

\subsubsection{Mature miRNA expression assays}

The levels of mature miR-132, -212, -134 were detected by using Taqman MicroRNA reverse transcription kit (Applied Biosystems, 4366596) and Taqman Universal PCR master mix with UNG (4304437, Applied Biosystems) according to manufacturer's protocol with some 
modifications. Sno-202 was used as endogenous control. The RT and Q-PCR primers for hsamiR-132, mmu-miR-212, mmu-miR-132 and sno-202 were obtained from Taqman MicroRNA assay kits (Applied Biosystems).

\subsection{Reverse transcription}

The RNA samples were diluted to the concentration of $24 \mathrm{ng} / 1$ with DEPC-treated $\mathrm{H}_{2} \mathrm{O}$. The RT reaction was prepared as the following:

Table 3: miRNA RT reaction protocol

\begin{tabular}{|l|l|}
\hline Component (stock concentration) & Volume to be used for 20 1 of RT reaction ( 1) \\
\hline dNTP $(100 \mathrm{mM})$ & 0.2 \\
\hline Reverse Transcriptase (50U/ 1) & 1.3 \\
\hline 10X reverse transcription buffer & 2 \\
\hline Rnase inhibitor (20U/ 1) & 0.25 \\
\hline DEPC-treated $\mathrm{H}_{2} \mathrm{O}$ & 3.9 \\
\hline
\end{tabular}

7.751 of RT master mix and 51 of RNA (120 ng) were mixed by tipping gently and centrifuged for $1 \mathrm{~min}$ at $2000 \mathrm{rpm} .7 .321$ of RT primer was added. The mixture was mixed by tipping and centrifuged at $2000 \mathrm{rpm}$ for $1 \mathrm{~min}$. The reaction mixture was kept on ice for $5 \mathrm{~min}$ and the reaction was performed with the thermal cycler that was programmed with the following parameter values.

Table 4: Thermal cycler program for miRNA RT reaction.

\begin{tabular}{|l|l|}
\hline Time (min) & Temperature $\left({ }^{\circ} \mathbf{C}\right)$ \\
\hline 30 & 16 \\
\hline 30 & 42 \\
\hline 5 & 85 \\
\hline$\infty$ & 4 \\
\hline
\end{tabular}

The synthesized cDNAs were stored at $-20^{\circ} \mathrm{C}$. 


\subsection{Q-PCR}

The master mix for Q-PCR reaction was prepared as the following:

Table 5: MiRNA specific Q-PCR reaction protocol

\begin{tabular}{|l|l|}
\hline Component & Volume to be used for 20 l of reaction ( I) \\
\hline Taqman Master mix & 10 \\
\hline Q-PCR primer & 1 \\
\hline $\mathrm{ddH}_{2} \mathrm{O}$ & 7.67 \\
\hline cDNA & 1.33 \\
\hline
\end{tabular}

The reaction mixture was transferred into wells of 96-well plate (twin.tec real time PCR plate, 0030132.530). Each reaction was performed in technical triplicates. The plate was loaded into the thermal cycler (Master cycler ep realplex, Eppendorf) with the following parameters.

Table 6: Thermal cycler program for miRNA specific Q-PCR

\begin{tabular}{|l|l|l|l|}
\cline { 2 - 4 } & Step & Temperature $\left({ }^{\circ} \mathbf{C}\right)$ & Time \\
\cline { 2 - 4 } & UNG enzyme activity & 50 & $2 \mathrm{~min}$ \\
\hline UNG enzyme inactivation & 95 & $10 \mathrm{~min}$ \\
\hline & cycles & 95 & $15 \mathrm{sec}$ \\
\hline & Denaturation & 60 & $1 \mathrm{~min}$ \\
\hline
\end{tabular}

The $\mathrm{Ct}$ values were calculated automatically by the built-in algorithm of the thermo cycler software. Quantitation of the miRNA expression was performed according to the comparative $\mathrm{C}_{\mathrm{t}}$ method.

\subsubsection{Gene expression assays}

BDNF, c-Fos and Arc transcripts were reverse transcribed by using first strand cDNA synthesis kit (GE Healthcare, 27-9261-01) according to the manufacturer's instructions. Taqman gene expression master mix (Applied Biosystems, 4369016) and Taqman gene expression assays (Applied Biosystems) were used to detect the expression levels of the transcripts. 


\subsection{Reverse transcription}

The RNA samples were diluted to the concentration of $40 \mathrm{ng} / 1$ with DEPC-treated $\mathrm{H}_{2} \mathrm{O}$ and incubated at $65^{\circ} \mathrm{C}$ for $10 \mathrm{~min}$ for denaturation. The $\mathrm{RT}$ reaction was prepared as the following:

Table 7: mRNA RT reaction protocol

\begin{tabular}{|l|l|}
\hline Component & Volume to be used for 33 I of RT reaction ( l) \\
\hline Primer $p d(N)_{6}$ & 1 \\
\hline Dithiothrietol (DTT) & 1 \\
\hline Reaction mix & 11 \\
\hline RNA & 20 \\
\hline
\end{tabular}

The program of the thermal cycler for RT reaction was as shown below.

Table 8: Thermal cycler program for mRNA RT

\begin{tabular}{|l|l|}
\hline Time & Temperature $\left({ }^{\circ} \mathbf{C}\right)$ \\
\hline $1 \mathrm{hr}$ & 37 \\
\hline $10 \mathrm{~min}$ & 98 \\
\hline
\end{tabular}

\subsection{Q-PCR}

The master mix for Q-PCR reaction was prepared as following:

Table 9: Q-PCR reaction formula for gene expression assay

\begin{tabular}{|l|l|}
\hline Component & Volume to be used for 20 l of reaction ( I) \\
\hline 2X Taqman Mastır Mix & 10 \\
\hline 20X Gene Expression Assay & 1 \\
\hline $\mathrm{ddH}_{2} \mathrm{O}$ & 7.6 \\
\hline cDNA & 1.4 \\
\hline
\end{tabular}


The reaction mixture was transferred into wells of 96-well plate. Each reaction was performed in technical triplicates. The plate was loaded into the thermal cycler (Master cycler ep realplex, Eppendorf) with the following parameters.

Table 10: Thermal cycler program for gene expression assay.

\begin{tabular}{|l|l|l|l|}
\hline Step & Temperature $\left({ }^{\circ} \mathbf{C}\right)$ & Time \\
\cline { 2 - 4 } & UNG enzyme activity & 50 & $2 \mathrm{~min}$ \\
\hline 40 cycles & UNG enzyme inactivation & 95 & $10 \mathrm{~min}$ \\
\hline & Denaturation & 95 & $15 \mathrm{sec}$ \\
\cline { 2 - 4 } & Annealing and extension & 60 & $1 \mathrm{~min}$ \\
\hline
\end{tabular}

The $\mathrm{Ct}$ values were calculated automatically by the built-in algorithm of the thermo cycler software. Quantitation of the miRNA expression was performed according to the comparative $\mathrm{C}_{\mathrm{t}}$ method.

\subsection{Materials}

\subsubsection{Buffers and solutions}

1X Artificial Cerebrospinal Fluid (ACSF)

- $124 \mathrm{mM} \mathrm{NaCl}$

- $1 \mathrm{mM} \mathrm{MgCl} 2$

- $4 \mathrm{mM} \mathrm{KCl}$

- $2 \mathrm{mM} \mathrm{CaCl}_{2}$

- $26 \mathrm{mM} \mathrm{NaHCO}_{3}$

- $1.25 \mathrm{mM} \mathrm{NaH}_{2} \mathrm{PO}_{4}$

- $10 \mathrm{mM}$ D-glucose

10 X Phosphate Buffered Saline (PBS)

- $\quad 1.36 \mathrm{M} \mathrm{NaCl}$

- $26 \mathrm{mM} \mathrm{KCl}$

- $42 \mathrm{mM} \mathrm{Na}_{2} \mathrm{HPO}_{4} \cdot 2 \mathrm{H}_{2} \mathrm{O}$

- $15 \mathrm{mM} \mathrm{KH}_{2} \mathrm{PO}_{4}$

- Stored at RT $\mathrm{pH}: 7.3$ 
1X Fixation Solution

- $1 \%$ formaldehyde

- $0.5 \%$ glutaraldehyde

- $0.2 \%$ NP40

- $0.1 \%$ Sodium deoxtcholate

- Dissolve in $1 \mathrm{X}$ PBS

- Prepared before use

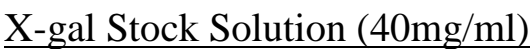

- Invitrogen, Cat No: 15520-018

- Dissolved in dimethyl formamide

- Stored at $-20^{\circ} \mathrm{C}$

$\underline{1 X \beta-G a l a c t o s i d a s e ~ S t a i n i n g ~ S o l u t i o n ~}$

- $2 \mathrm{mM} \mathrm{MgCl} 2$

- $0.02 \% \mathrm{NP} 40$

- $0.01 \%$ Sodium deoxycholate

- $2.5 \mathrm{mM} \mathrm{K}_{3}\left[\mathrm{Fe}(\mathrm{CN})_{6}\right]$

- $2.5 \mathrm{mM} \mathrm{K}_{4}\left[\mathrm{Fe}(\mathrm{CN})_{6}\right]$

- $1 \mathrm{mg} / \mathrm{ml} \mathrm{X-gal}$

- Dissolved in PBS

- Prepared before use

1X LacZ Buffer

- $2 \mathrm{mM} \mathrm{MgCl}_{2}$

- $0.02 \% \mathrm{NP} 40$

- $0.01 \%$ sodium deoxycholate

- Dissolved in PBS

- Stored at RT

Mounting Medium

- Moviol

- Get the protocol from Sabine

\section{$\underline{\text { DEPC-treated } \mathrm{H}_{2}} \underline{\mathrm{O}}$}

- $0.1 \%$ DEPC

- Dissolved by shaking at $37^{\circ} \mathrm{C}$ for o/n

- Autoclaved 
- $10 \mathrm{mM}$ Tris

- $0.1 \mathrm{mM}$ EDTA

- $0.5 \%$ SDS

- After autoclaving acidic phenol was added to have $1 \%$ final concentration.

GTC Denaturating Solution

- 4 M Guanidin thiocyanate (GTC)

- $0.02 \mathrm{M}$ Tri-sodium citrate dehydrate

- $0.5 \%$ Sarcosyl

- Add 1.5 fold less DEPC-treated $\mathrm{H}_{2} \mathrm{O}$ than the final volume

- Dissolve by stirring for o/n at $37^{\circ} \mathrm{C}$.

- Complete the volume to the final volume.

$\underline{\text { Trizol }}$

- $50 \mathrm{ml} \mathrm{GTC}$ denaturating solution (final volume $105 \mathrm{ml}$ )

- $0.01 \mathrm{M} \mathrm{NaOAc}$

- $50 \mathrm{ml}$ acidic phenol (final volume $105 \mathrm{ml}$ )

- $0.05 \mathrm{M} \beta$-mercaptoethanol (14.3 $\mathrm{M}$ is the stock concentration)

- Mix well and keep at $4^{\circ} \mathrm{C}$ in dark bottle.

1X Lysis Buffer

- $50 \mathrm{mM}$ Tris- $\mathrm{HCl}$ (pH: 7.4)

- $1 \%$ NP40

- $0.25 \% \mathrm{NaDOC}$

- $150 \mathrm{mM} \mathrm{NaCl}$

- $1 \mathrm{mM}$ EDTA

- Store at $4^{\circ} \mathrm{C}$

- Just before use, protease inhibitor (cOmplete, EDTA-free, Roche, 11873580001) and phosphatase inhibitor (PhosSTOP, Roche, 04906845001) tablets were dissolved in $10 \mathrm{ml}$ of lysis buffer.

\section{$\underline{\text { 5X SDS Loading Buffer }}$}

- $143 \mathrm{mM}$ Tris- $\mathrm{HCl}(\mathrm{pH}: 6.8)$

- $286 \mathrm{mM}$ DTT

- $4.3 \mathrm{mM}$ bromophenol blue

- $14.2 \%$ Glycerol

- $200 \mathrm{mM}$ SDS

- Dissolved in $\mathrm{ddH}_{2} \mathrm{O}$ by rotating at $37^{\circ} \mathrm{C}$ for o/n. Aliquoted and stored at $-20^{\circ} \mathrm{C}$. 
$10 \%$ SDS-Page Resolving Gel

- $10 \%$ acrylamide mix (Rotiphorese Gel 30, 37.5:1, Roth, 3029.2)

- 375 mM Tris (pH: 8.8)

- $0.1 \%$ SDS

- $0.1 \%(w / v)$ ammonium persulfate

- $2.7 \mathrm{mM}$ Tetramethylethylenediamine (TEMED)

- Dissolved in $\mathrm{ddH}_{2} \mathrm{O}$. Mixed vigorously and poured into $1 \mathrm{~mm}$ BioRad MiniProtean gel system. The upper layer was covered with isopropanol to flatten the surface. The gel was let for freezing at RT for $15 \mathrm{~min}$. Then the isopropanol was discarded and the residual isopropanol was cleaned by washing with $\mathrm{dH}_{2} \mathrm{O}$.

SDS-Page Stacking Gel

- $5.1 \%$ acrylamide mix

- 125 mM Tris (pH: 6.8)

- $0.1 \% \mathrm{SDS}$

- $0.1 \%(\mathrm{w} / \mathrm{v})$ ammonium persulfate

- $6.7 \mathrm{mM}$ TEMED

- Dissolved in $\mathrm{ddH}_{2} \mathrm{O}$. Mixed vigorously and poured onto the $10 \%$ SDS-Page resolving gel. Immediately after the gel comb was placed. The gel was let for freezing for $15 \mathrm{~min}$.

10X TBS

- $\quad 1.37 \mathrm{M} \mathrm{NaCl}$

- $27 \mathrm{mM} \mathrm{KCl}$

- 250 mM TRIS

- $\mathrm{pH}$ was set to 7.4 and autoclaved.

- Dilute 10X TBS by 10 folds

- Add Tween20 to have final concentration of $0.1 \%$.

$\underline{10 X \text { Tris-Glycine Electrophoresis Buffer }}$

- $250 \mathrm{mM}$ TRIS

- 2.5 M Glycine

- $1 \%$ SDS

- The mixture was dissolved in $\mathrm{ddH}_{2} \mathrm{O}$ at $37^{\circ} \mathrm{C}$ for $2-3$ hours by stirring

- The $\mathrm{pH}$ was set to 8.3

10X Transfer Buffer

- 386 mM Glycine

- $480 \mathrm{mM}$ TRIS

- $13 \mathrm{mM}$ SDS 
- The mixture was dissolved in $\mathrm{ddH}_{2} \mathrm{O}$.

- The $\mathrm{pH}$ was set to 8.3

\subsubsection{Cell Culture Medium}

\section{DMEM/10\% FBS}

- 450 ml DMEM-High Glucose, No glutamine (Gibco, 11960-044)

- $50 \mathrm{ml}$ heat inactivated 10\% FBS (Hyclone-Thermo Scientific, SH30070.01HI)

- Aliquoted and stored at $4{ }^{\circ} \mathrm{C}$.

Culture medium

- $500 \mathrm{ml} 1 X$ Neurobasal Medium (Gibco, 21103-049

- $10 \mathrm{ml}$ of 50X B27 serum-free supplement (Gibco, 17504-044)

- $1.25 \mathrm{ml}$ of $200 \mathrm{mM}$ L-Glutamine (Final Concentration $0.5 \mathrm{mM}$ ) (Gibco, 25030)

- Aliquoted and stored at $4{ }^{\circ} \mathrm{C}$

\subsubsection{Drugs}

$\underline{\text { Kainic acid }}$

- 5mg kainic acid (Santa Cruz, sc-200454) was dissolved in $1 \mathrm{ml}$ of $\mathrm{ddH}_{2} \mathrm{O}$ to obtain $23.45 \mathrm{mM}$ stock solution.

- Aliquoted and stored at $-20^{\circ} \mathrm{C}$ in dark.

\section{Forskolin}

- $10 \mathrm{mg}$ forskolin (Cell Signaling, 3828) was dissolved in 8121 of DMSO to obtain $30 \mathrm{mM}$ stock solution.

- $\quad$ Stored at $-20^{\circ} \mathrm{C}$ in dark. 


\subsubsection{Antibodies}

Table 11: Primary antibodies used for western blotting experiments.

\begin{tabular}{|l|l|l|l|}
\hline Primary Antibodies & Dilution & Blocking & Manufacturer and Catalog \# \\
\hline Mouse monoclonal CREB & $1 / 500$ & Milk & Cell Signaling \\
\hline Rabbit anti-mouse polyclonal pCREB & $1 / 1000$ & BSA & Cell Signaling, 91918 \\
\hline Rabbit anti-mouse polyclonal SIRT1 & $1 / 1000$ & Milk & Millipore, 07131 \\
\hline Mouse monoclonal $\alpha$-TUBULIN & $1 / 10000$ & Milk/BSA & Sigma, T6199 \\
\hline Rabbit anti-mouse polyclonal MecP2 & $1 / 500$ & BSA & Millipore, 07-013 \\
\hline $\begin{array}{l}\text { Rabbit anti-mouse polyclonal } \\
\text { P250GAP }\end{array}$ & $1 / 1000$ & BSA & Abgent \\
\hline
\end{tabular}

Table 12: Secondary antibodies used for western blotting experiments.

\begin{tabular}{|l|l|l|}
\hline Secondary Antibodies & Dilution & Manufacturer and Catalog \# \\
\hline ECL, Rabbit IgG, HRP-Linked Whole $2^{\circ} \mathrm{Ab}$ (from donkey) & $\underline{1 / 20000}$ & $\underline{\text { GE Healthcare }}$ \\
\hline ECL, Mouse IgG, HRP-Linked Whole $2^{\circ} \mathrm{Ab}$ (from sheep) & $\underline{1 / 10000}$ & $\underline{\text { GE Healthcare }}$ \\
\hline
\end{tabular}

\subsubsection{Gene and miRNA Expression Assays}

Gene expression assays (Applied Biosystems)

Table 13: Gene expression assay ID numbers.

\begin{tabular}{|l|l|}
\hline Assay Name & Assay ID \\
\hline c-Fos & Mm00487425_m1 \\
\hline Arc & Mm00479619_g1 \\
\hline BDNF & Mm04230607_s1 \\
\hline Zif268 & Mm00656724_m1 \\
\hline Gapdh & Mm99999915_g1 \\
\hline
\end{tabular}

MiRNA expression assays (Applied Biosystems) 
Table 14: miRNA expression assay ID numbers.

\begin{tabular}{|l|l|l|}
\hline RT primer & $\begin{array}{l}\text { Volume to be used for } \\
201 \text { of reaction ( 1) }\end{array}$ & Assay ID \\
\hline miR-132 & 1.83 & 000457 \\
\hline miR-212 & 1.83 & 002551 \\
\hline miR-134 & 1.83 & 001186 \\
\hline sno-202 & 1.83 & 001232 \\
\hline
\end{tabular}




\section{RESULTS}

\section{1. miR-212/132 mull mutant mice longevity and body weight}

As stated in an earlier study, miR-212/132 null mutant (miR-212/132--) mice are viable and fertile ${ }^{115}$. To check if there is any metabolic defect that in turn may affect the body weight of mice, male mice at different ages between 40 and 289 days old were weighed. The agedependent weight gain of miR-212/132/- mice was similar as their wild type (WT) littermates. There was no difference in the body weights of miR-212/132/- and WT mice at any age groups analyzed (Fig 3). Therefore miR-212/132 deletion does not alter the body weight of mice.

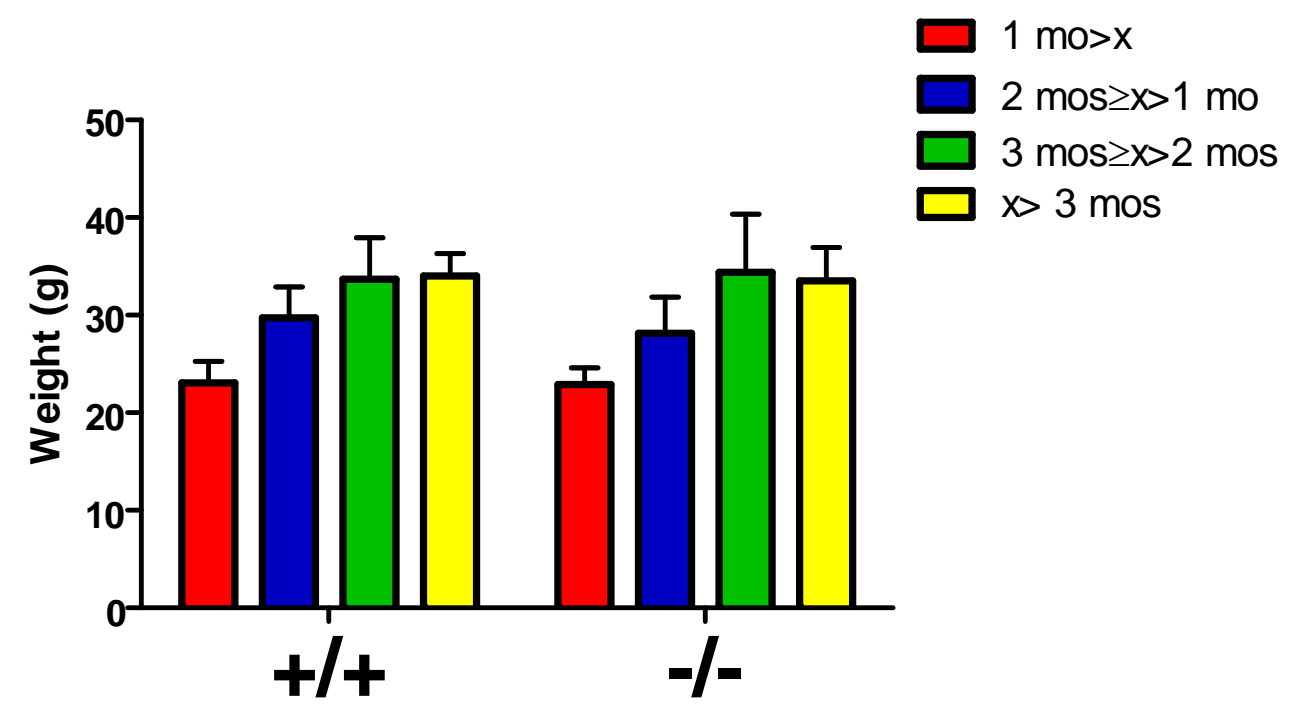

Figure 3: Body weight measurement of WT and miR-212/132 $2^{-/-}$mice at different ages. The mice were grouped according to their ages as younger than 1 month, 1-2 months old, 2-3 months old and older than 3 months. 2way ANOVA for age versus genotype interaction $F_{(3,59)}=0.34, p=0.8$; for difference between mice at different age groups $F_{(3,59)}=26.4$, $p<0.0001$; for difference between genotypes $F_{(1,59)}=0.18, p=0.67: 1 \mathrm{mo}>x$ : $+/+, n=4,-/-, n=8 ; 2 \operatorname{mos}>x>1 \operatorname{mos}: ~:+/+, n=16,-/-, n=11 ; 3 \operatorname{mos}>x>2 \operatorname{mos}:+/+, n=10,-/-, n=5 ; x>3 \operatorname{mos}:+/+, n=8,-$ $/-, \mathbf{n}=\mathbf{5}$.

The possible loss-of-function phenotype of miR-212/132 on the longevity was studied by keeping the mice in house cages in a room at $24^{\circ} \mathrm{C}$ with $12 \mathrm{~h}$ light-dark cycle and ad libitum access to food and water. The health status of these mice was checked regularly. If any symptom causing pain or sign of tumor formation were observed, the mice were sacrificed immediately and the date of killing was recorded. Eight WT mice out of 18 (44\%) died during the course of 
the experiment and 10 of them (56\%) were observed to develop tumor at old ages. Eleven out of $29 \mathrm{miR}-212 / 132^{-/-}$mice $(37 \%)$ died during the longevity experiments and tumor formation was detected in 18 of them (62\%) (Fig. 4b). However Fisher's exact test revealed that genotype of mice is not a determinant factor for tumor formation or death due to any other reason.

a

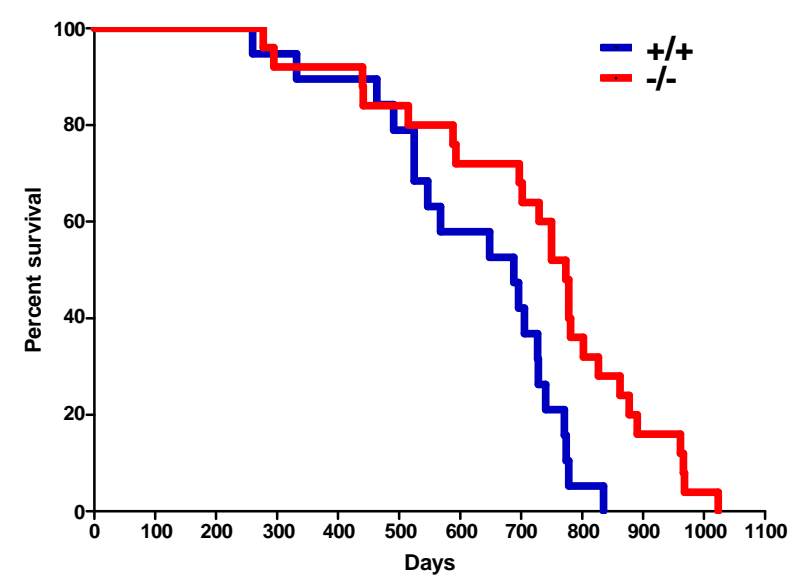

b

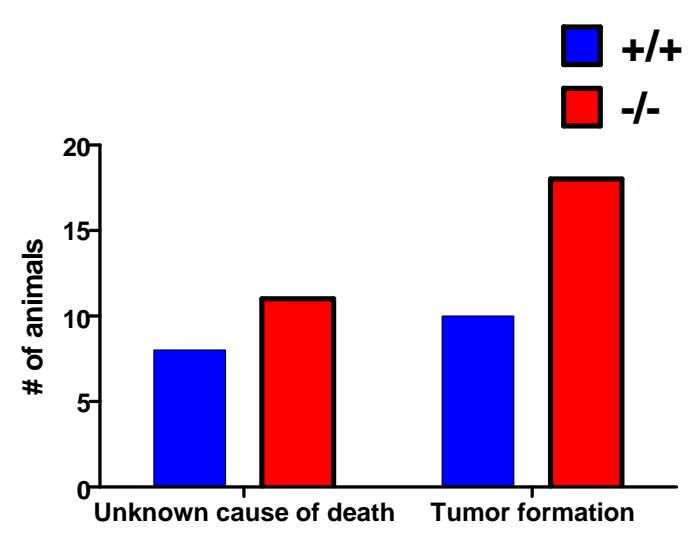

Figure 4: Survival analysis on miR-212/132 ${ }^{-/}$mice. The health status of WT and miR-212/132 ${ }^{-/-}$mice with ad libitum access to water and food were observed continuously. (a) The date of death of each mouse was recorded and (b) the presence of tumors was evaluated as the cause of death. If an obvious tumor formation was observed, the mice were classified in "tumor formation" group. If the mice were found death in cage or tumor formation was not observed they were grouped as "unknown cause of death". (a) Mantel-Cox test, p=0.0038; Gehan-Breslow-Wilcoxon test, p=0.02. (b) Fisher's exact test, two-sided, p=0.76.

The life expectancy of miR-212/132/- mice compared to WT littermate controls was analyzed by Kaplan-Meier survival assay. According to Mantel-Cox test the longevity of miR$212 / 132^{-/-}$is significantly higher than the control mice (Fig. 4a). The median survival duration for miR-212/132/- is 773 days while control mice survive for 688 days. The hazard ratio is 2.933 , which means that the rate of death of WT mice is 2.933 -fold more than the miR-212/132-/mice. Those data implies that miR-212/132 may have an important functional role in the determination of the life-span of mice. 


\subsection{Expression of miR-212/132 in various organs of mouse}

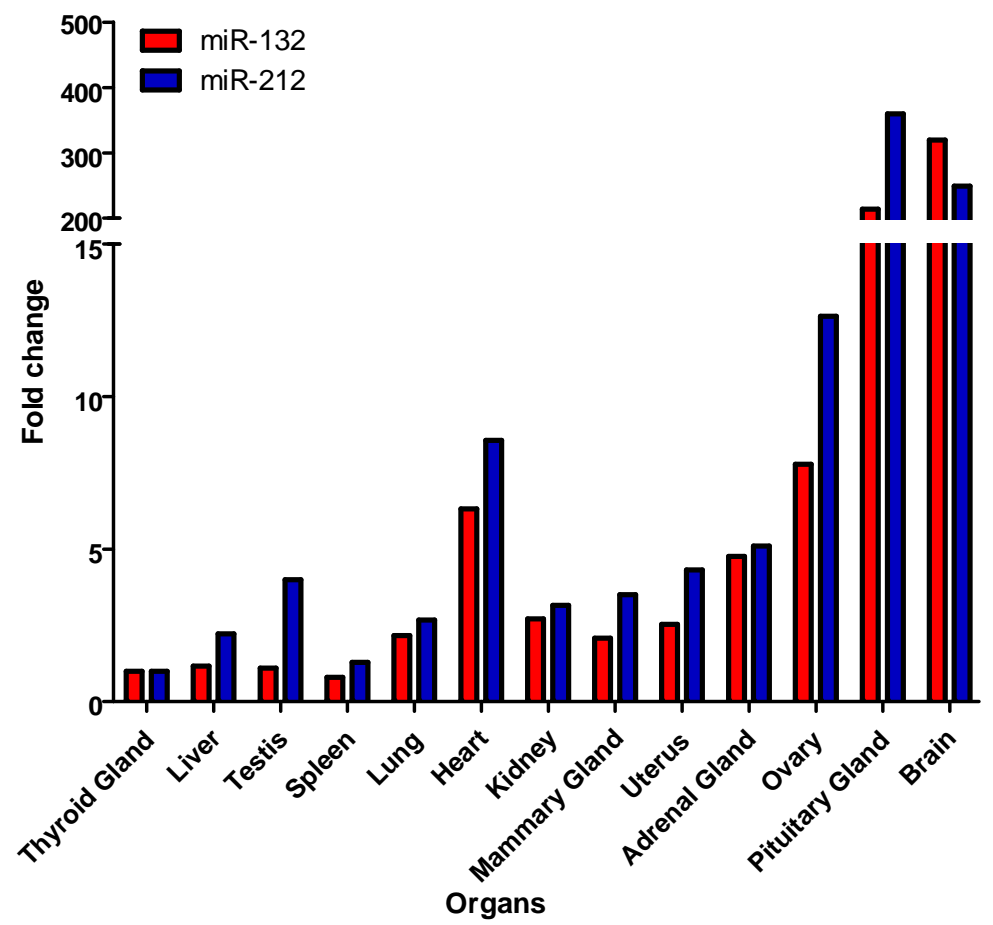

Figure 5: The levels of mature miR-212 and miR-132 in different mouse organs. The organs were dissected and RNA isolation was performed. Equal concentration of cDNA was used to perform RT Q-PCR. Sno202 was used as endogenous control. The miR-212 and miR-132 levels in thyroid gland were set to 1 and the fold expressions were calculated accordingly.

MiR-132 was first defined as a brain specific miRNA ${ }^{111}$ and miR-212 was detected in HeLa cells ${ }^{110}$. To study the expression of miR-212/132 in various mouse organs, total RNAs from thyroid gland, mammary gland, adrenal gland, pituitary gland, liver, testis, spleen, lung, heart, kidney, uterus, ovary and brain were isolated. MiR-212 and miR-132 specific Q-RT PCRs were performed (Fig. 5). The fold difference of miR-212 and miR-132 expression levels were plotted in comparison to their levels in the thyroid gland, in which the relative levels of miR-212 and miR-132 were lowest and therefore were set arbitrarily as 1 . The expression levels of miR132 and miR-212 are roughly similar in spleen, lung, kidney, and adrenal gland. MiR-212 levels are higher than miR-132 in liver, testis, heart, mammary gland, ovary and pituitary gland. In contrary higher miR-132 expression levels were detected in brain compared with miR-212 levels. The strongest expression of both miR-212 and miR-132 were observed in the brain and pituitary gland. 
This data shows that miR-212 and miR-132 are expressed strongly in brain and pituitary gland as well as in peripheral organs albeit in lower levels. The high levels of expression in pituitary gland and brain may correlate with their potential functional roles in those tissues. Nevertheless, relatively lower expression levels in other organs do not necessarily exclude any potential roles of miR-212/132 in those tissues.

\section{3. miR-212/132 expression studies in brain}

Earlier studies using RT PCR showed that miR-212/132 is expressed in neurons and its expression is induced by neuronal activity. However knowledge on its spatiotemporal expression pattern is necessary in order to be able to predict and analyze the possible functions of miR212/132 appropriately. The widely used method for studying the spatial expression of a gene on tissue sections is in situ hybridization (ISH). However the small size of mature miRNAs leads to difficulties in designing efficient ISH probes. Therefore, locked nucleic acid (LNA) probes have been used to increase the specificity and sensitivity of the assay. Different LNA probes against miR-212 and miR-132 were used to study its expression in adult brain sections. However no specific binding of the LNA probes were detectable (data not shown). Therefore ISH could not be used as a successful method to study the spatial expression pattern of miR-212/132 at histological level.

As an alternative approach, the knocked-in B-Galactosidase gene in the miR-212/132/mouse line ${ }^{115}$ was used to follow the spatiotemporal expression of miR-212/132 in the brain. For the generation of the deletion mutant, only $900 \mathrm{bp}$ of the expressed sequence of the non-coding transcript that encompasses both miRNAs was deleted. It can therefore be assumed that the regulatory sequences in the miR-212/132 loci are intact and would drive the expression of the inserted lacZ sequence mimicking the expression of miR-212/132.

\subsubsection{Arguments to utilize miR-212/132-- mice for $\beta$ - galactosidase staining}

Detailed analyses of histological sections of WT and heterozygous as well as homozygous mutant brain failed to reveal any overt structural phenotypes. $\beta$-galactosidase staining was first performed on tissue sections of mice that were heterozygous for the knock-in 
LacZ reporter gene (miR-212/132 $\left.{ }^{+-}\right) .50 \mathrm{~m}$ thick coronal brain sections which were incubated in LacZ staining solution at $37^{\circ} \mathrm{C}$ for $72 \mathrm{hrs}$ stained positively, but rather weakly. This approach might therefore exclude the detection of brain regions that are expressing the miR-212/132 at a low level. Since the miR-212/132 $2^{-/}$did not have any overt structural phenotype in the brain (data not shown), to obtain a stronger $\beta$-Galactosidase staining brain sections from homozygous mutant brains were then utilized. The staining pattern on brain samples of miR-212/132 $2^{-/-}$and miR-212/132 $2^{+/}$mice were compared in order to see if there is any significant alteration in the spatial distribution of the staining (Fig. 6). On the whole mount staining of miR-212/132 $2^{+-}$and miR-212/132 $2^{-/}$brains, the staining at dorsal side can be observed along the cortex, and relatively low at retrosplenial area (RSP) (Fig. 6a, left). On the ventral side the staining is detectable at hippocampal formation (HPF) and taenia tecta (TT) (Fig. 6a, right). The brain regions where the staining is most intensive were selected as representative planes to demonstrate the levels of staining at deeper structures (Fig. 6b, c). As expected, the staining in cerebral cortex of the homozygous miR-212/132/- brain was much stronger than the cortex of the heterozygous miR$212 / 132^{+/-}$mouse brain. Nevertheless, the expression pattern in both genotypes overlapped appropriately (Fig. 6a). Dense population of cells stained in DG, CA1 and CA3 regions are visible in miR-212/132/- dorsal hippocampus. On the other hand, the sections from the heterozygous mutant brains also had the staining in DG, CA1 and CA3 region as well, even though staining levels were weaker (Fig. 6d, e). 

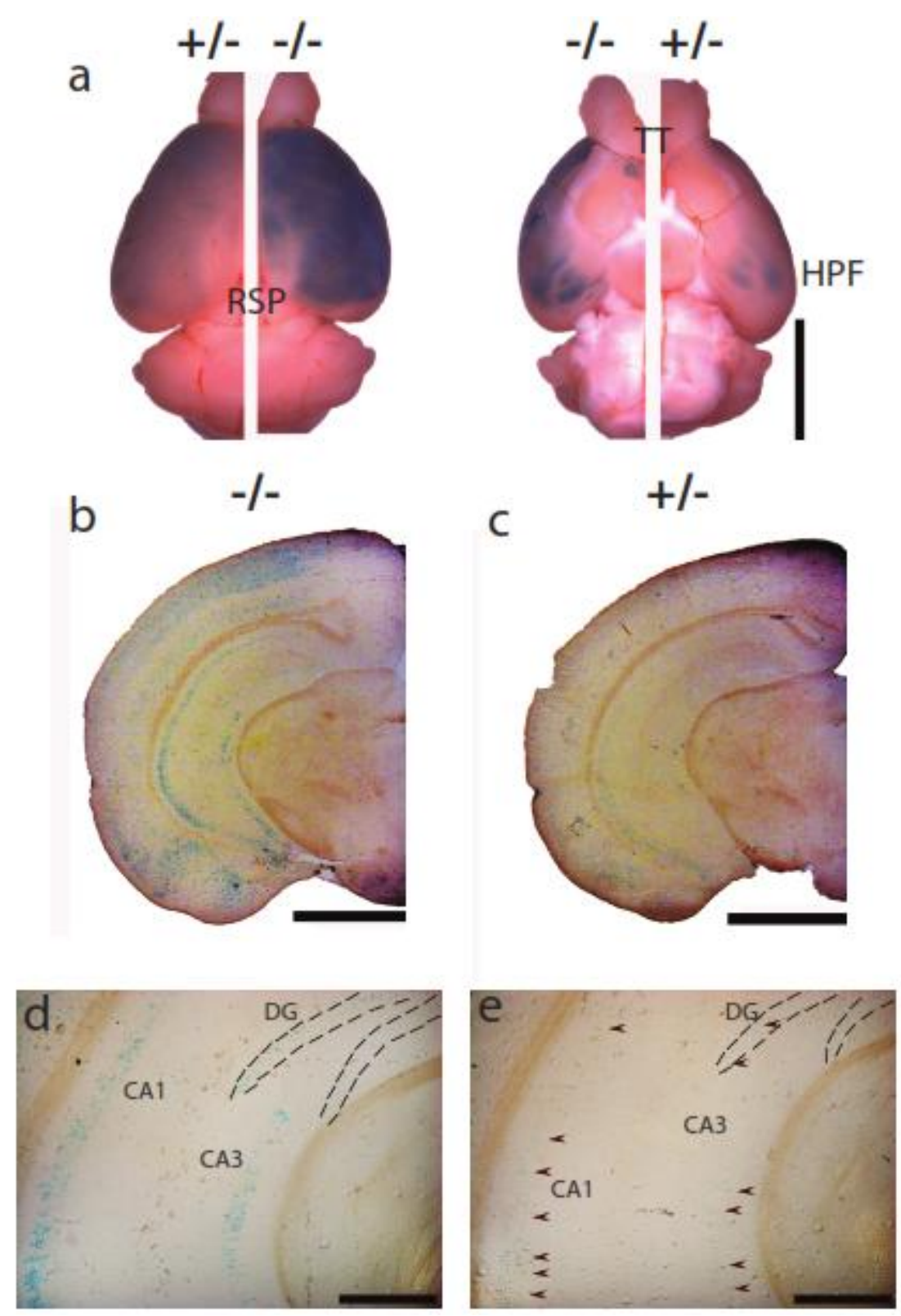

Figure 6: The staining patterns on miR-212/132 $2^{+-}$and miR-212/132 ${ }^{-/}$brains were compared. (a) Whole mount staining of brains of +/- and -/- mice were visualized from dorsal and ventral sides. RSP: retrosplenial area, TT: taenia tecta, HPF: Hippocampal formation. (b,c) The brain sections, where $\beta$-galactosidase staining was most prominent, were shown here as representative planes. (d, e) Higher magnification pictures on dorsal hippocampus of the sections shown in (b) and (c). The arrows point dotted staining. Scale bars represent $5 \mathrm{~mm}$ in (a), $2 \mathrm{~mm}$ in $(b, c)$ and $500 \mathrm{~m}$ in $(d, e)$.

\subsubsection{Spatial expression of miR-212/132 in brain}

To analyze the expression of miR-212/132 in the brains of young adolescent mice, brains of 5 weeks old mice were dissected and sectioned for $\beta$-galactosidase staining. The LacZ 
expression in the cortical plate exhibits distinct layer specificity (Fig. 7a-c). Abundant expression in the layers 2/3, 4 and 6 were observed. The expression in layer 5 is relatively lower than the other layers. The layer 1 is mostly occupied by axons that are projected from thalamus and other cortical layers and tuft dendrites of layer $2 / 3$ and 5 neurons ${ }^{290,291}$. Layer 1 is remarkable with sparseness of neurons. The existing neurons in this layer are exclusively inhibitory neurons ${ }^{292}$. No LacZ expression was detected in layer 1 (Fig. 7c). However since the $\beta$-galactosidase locates at the periphery of the nucleus and does not migrate through axons or dendrites, the absence of staining in layer 1 does not rule out the possible localization of miR-212/132 at layer 1 synapses.

Strong LacZ expression was detectable in the hippocampus. The highest expression was observed in the CA3 region (Fig. 7d, f). Only some neurons scattered throughout the pyramidal layers of $\mathrm{CA} 1$ and CA2 were stained for $\beta$-galactosidase activity (Fig. 7e). The spatial distribution of LacZ-positive neurons in CA1 and CA2 regions showed variability in different mice. Since miR-212/132 is a neuronal activity regulated gene, therefore the LacZ-positive neurons might reflect the subset of neurons that show activity at the time of dissection. LacZ expression in certain restricted regions of dorsal striatum and lateral amygdala (LA) were also observed (Fig. 7h,i). 

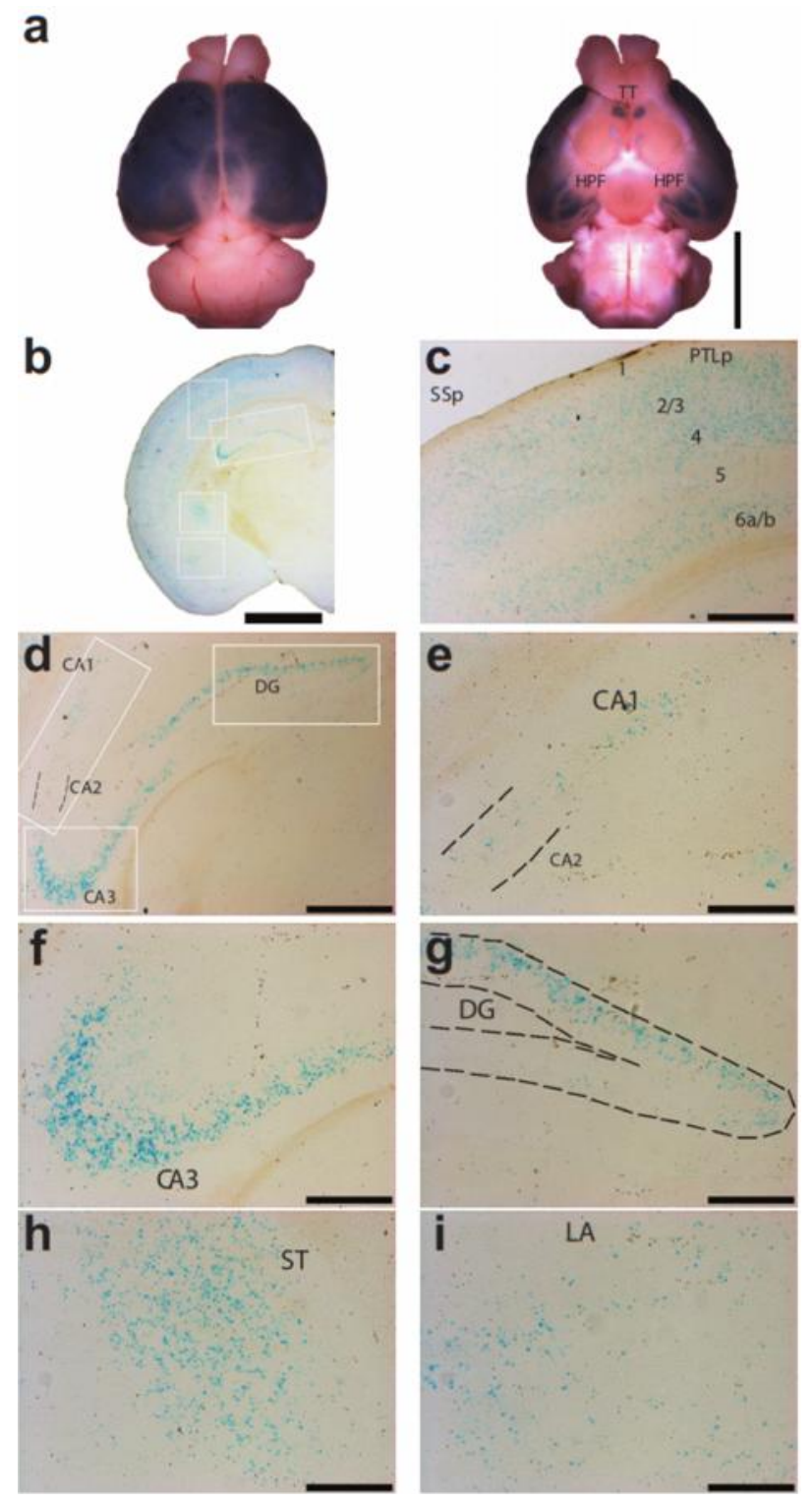

Figure 7: $\beta$-galactosidase staining on whole adult brain and coronal sections. (a) Whole mount $\beta$-galactosidase staining of 5 weeks-old mouse brain showed miR-212/132 expression in cortical plate. (b-i) $\beta$-galactosidase staining on coronal sections demonstrated LacZ expressing cells in spatially restricted regions of the forebrain (b), which is shown in higher magnification for somatosensory cortex (c), hippocampus (d), CA1 and CA2 regions of hippocampus, CA3 region of hippocampus (f), dentate gyrus (g), striatum (h) and amygdala (i). (c),(d), (h) and (i) are higher magnification pictures of the regions shown within white rectangles in (b). The regions shown within white rectangles in (d) are shown in high magnification in (e), (f), and (g). TT, taenia tecta; SSp, primary somatosensory area; PTLp, posterior parietal association areas; CA, cornu ammonis; DG, dentate gyrus; ST, striatum; LA, lateral amygdalar nucleus. The histological boundaries for cortical layers and hippocampal structures are determined according to the DAPI staining results of the same sections. Scale bars represent $5 \mathrm{~mm}$ in (a), $2 \mathrm{~mm}$ in (b), $500 \mu \mathrm{m}$ in (c) and (d), $200 \mu \mathrm{m}$ in (e-i).

Dentate gyrus (DG) showed differential expression pattern of the $\beta$-galactosidase activity at the anteroposterior axis. The LacZ expression was detected at both dorsal and ventral arms of 
the DG in the dorsal hippocampus. However the restriction of the expression to the dorsal arm of the DG was observed in more posterior brain sections (Fig. 8a-e, f-j). Additionally no staining was detected in the subgranular zone of DG. Subgranular zone is the region where the neuronal progenitors reside $293-295$ (Fig. 7g, Fig. 8). This data confirms the previous finding that miR$212 / 132$ expression increases as the granule neurons differentiate into mature neurons ${ }^{160}$. The staining pattern in hippocampus is similar in both genders (Fig. 8a-e, f-j).

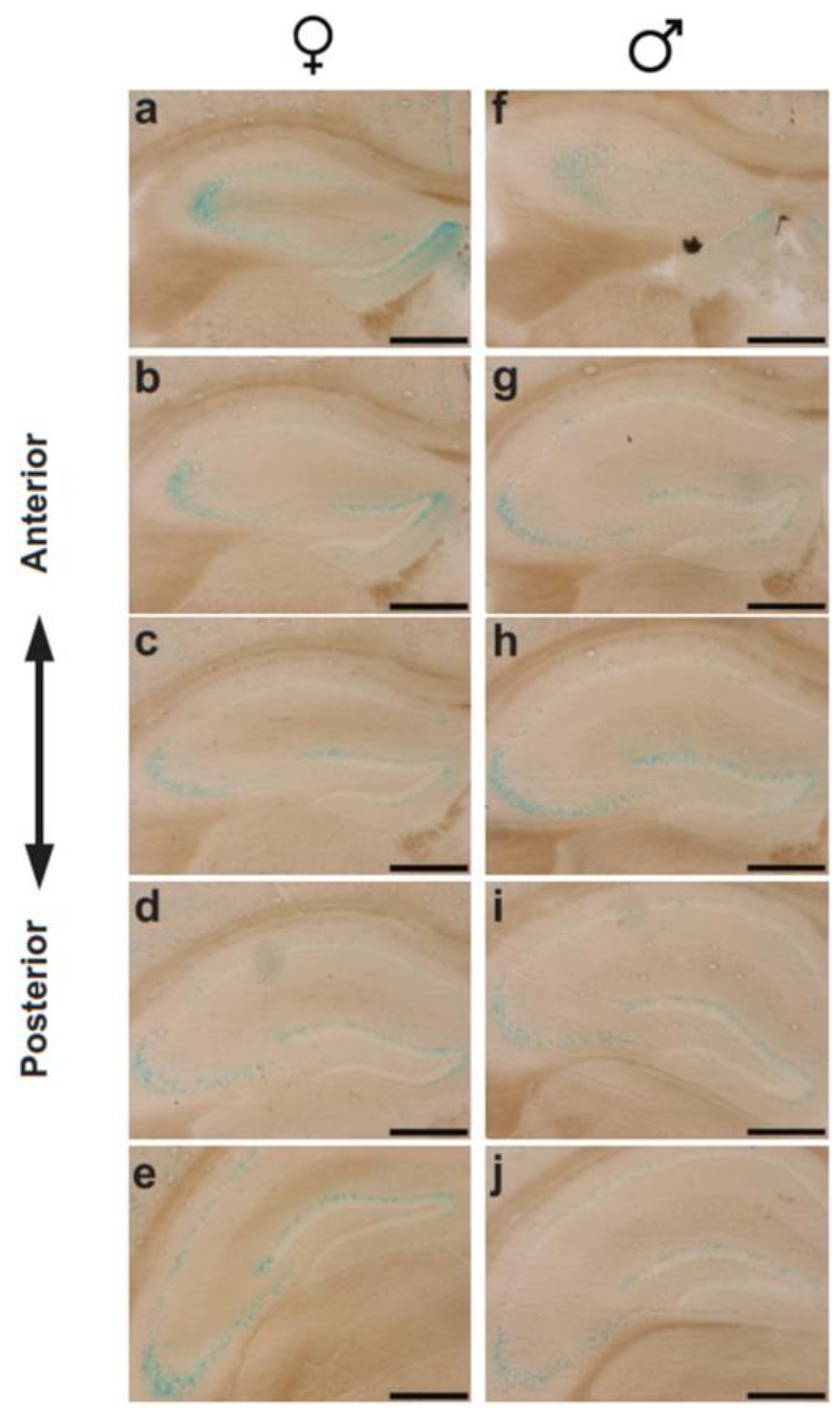

Figure 8: LacZ staining on the coronal sections of different planes of 5-weeks old male (a-e) and female (f-j) miR-212/132 ${ }^{-/-}$mouse brains demonstrated that mir-212/132 is expressed on both dorsal and ventral arms of the dentate gyrus in the anterior hippocampus, whereas its expression gets restricted to only dorsal arm of the dentate gyrus in the posterior hippocampus. Scale bars represent $500 \mu \mathrm{m}$. 
To verify that the detected $\beta$-galactosidase gene expression reflects the existence of mature miR-212 and miR-132, tissue samples from the thalamus, the CA3 and the cortex were dissected and total RNA from these samples were isolated. Quantitative RT-PCR was performed with probes specific to mature miR-132 and miR-212 sequences. The fold differences in miR212 and miR-132 levels in the CA3 and the cortex were calculated in comparison to their levels in the thalamus. The results for representative 2 independent experiments (depicted as A and B) are presented in fig. 9. MiR-132 and miR-212 levels in the CA3 region are slightly higher than their expression levels in the thalamus while the miR-132 and miR-212 expression levels are approximately 8 and 6 folds higher in cortex compared to their levels in the thalamus, respectively. In all these analyzed tissues miR-132 levels are higher than miR-212 levels, which is consistent with the results obtained for whole brain (Fig. 5). The obtained expression levels in the CA3 were lower than expected. CA3 is a very small area in the brain compared to cortex or thalamus. Therefore, there might have been some contamination from other tissues in its peripheries during dissection. In that case, the levels of miR-212 and miR-132 might be diluted and thus reflected as lower miRNA expression levels. Nevertheless, these results support that the $\beta$-galactosidase gene expression is correlated with the expression of mature miR-212 and miR132.

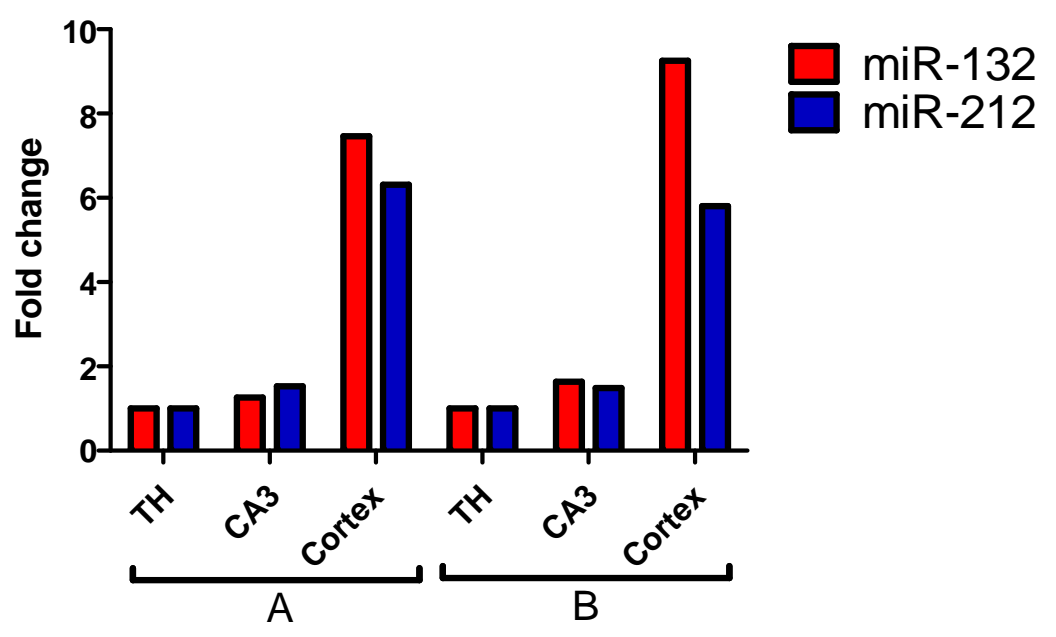

Figure 9: Relative expression levels of mature miR-212 and miR-132 in thalamus (TH), CA3 and cortex. One mm thick brain sections obtained from the section plane that corresponds to the dorsal hippocampus. Samples from TH, CA3 and cortex were dissected by Harris tissue corer for quantitative RT-PCR ${ }^{296}$. The relative fold change was calculated according to $\mathrm{TH}$ by setting the TH values to 1 . A and $\mathrm{B}$ represents two individual mice. 


\section{4. miR-212/132-/- mouse behavioral assays}

The expression of miR-212/132 in the cerebral cortex, hippocampus, amygdala and striatum implies that miR-212/132 might have role(s) in the regulation of behavior. However there are so far no studies that conducted wide array of behavioral assays using the miR-212/132 knockout, knockdown or overexpression experimental mouse model systems. The behavioral assays that were conducted in this work on miR-212/132/- and wild-type control mice evaluated their explorative activity, anxiety levels, sensorimotor gating, sociability, motor balance, coordination, conditioned learning, working memory and spatial memory.

\subsubsection{Anxiety levels, explorative activity, motor balance and coordination of miR-212/132/- mice}

Mice explore its environment for the sake of optimal survivability. However they prefer not to be in an open space, which otherwise would make them accessible for the predators. Consequently, they avoid any open space as much as possible during their explorative activities. Elevated plus maze consist of a plus-shaped lifted maze with two arms that are exposed and two closed arms. This test measures the amount of time spent in each arm. It assesses the tendency of mice to hide or explore and the results can be correlated with the level of anxiety. The amount of time that miR-212/132 ${ }^{-/-}$mice spent in the open arm, closed arm or at the center of the maze is not different than the WT control mice (Fig. 10a), indicating that the levels of anxiety is not impaired due to the loss-of- miR-212/132 function. Furthermore, the total distance covered on the maze is similar in both genotype groups, which suggests that the motor activity or the motivation to explore the arms is not altered in miR-212/132-/- mice (Fig. 10b).

The rotarod is a turning drum and the mice can run on it. As the drum accelerates, the mouse eventually falls. Quantifying the latency to fall assesses the motor balance and coordination of the mouse. The falling latency of miR-212/132/- and WT mice were not significantly different (Fig. 10c). The same test was performed on the second day to measure the motor learning. Both groups of mice stayed on the rotarod longer and falling latencies were still not different (Fig. 10c). These results demonstrate that the motor learning, balance and coordination of miR-212/132/- mice are not altered. 
The hole-board test is used to quantify the number of head dipping into the holes as an indication for directed exploration and locomotor activity ${ }^{192,297}$. The number of times that miR212/132 $2^{--}$and WT mice dip their head into the holes were not significantly different (Fig. 10d). As it was also demonstrated by the elevated plus maze assay, this data shows that the absence of miR-212/132 does not alter the exploratory behavior and locomotor activity of mice.

A

\section{Alevated plus maze: Time in zones}

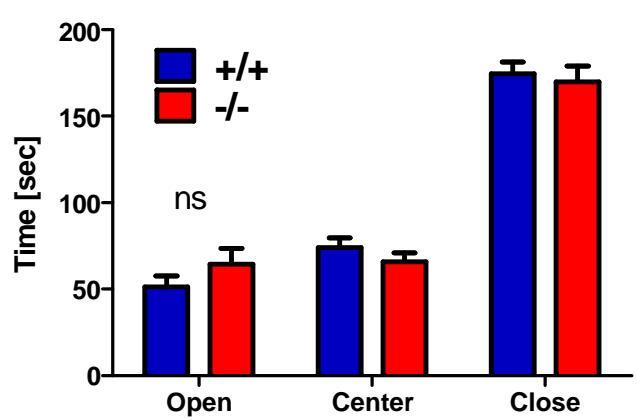

C

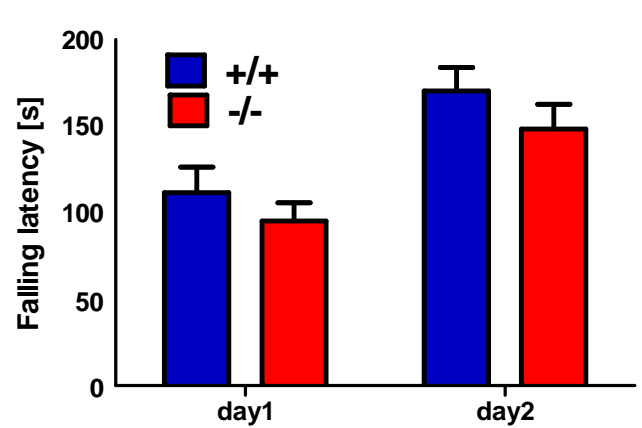

B

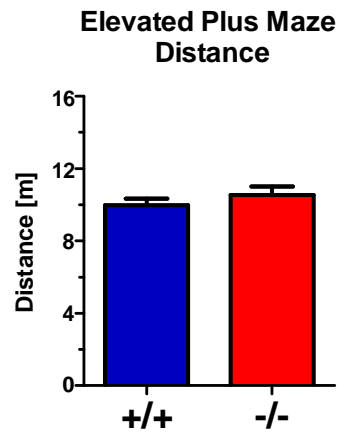

D

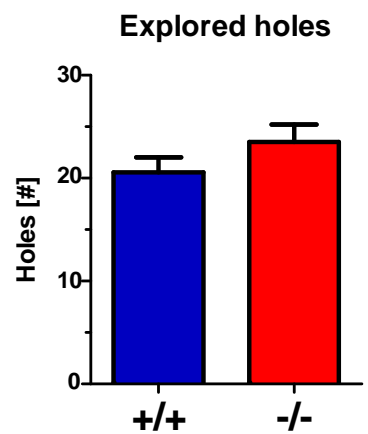

Figure 10: Elevated plus maze, rotarod and explored hole assays on WT and miR-212/132 ${ }^{-/-}$mice. (a, b) In the elevated plus maze test, times spent in different zones (a) and the total distance traveled (b) are similar between miR-212/132 ${ }^{-/-}$and WT littermates. (c) In the rotarod test, miR-212/132 ${ }^{-/-}$mice had similar falling latencies in both days compared to the WT controls. (d) In the hole-board test, the number of explored holes by the miR-212/132 ${ }^{-/-}$mice is similar to their WT mice. These experiments were conducted by Dr. Konstantin Radyushkin in the research group of Prof. Dr. Dr. Hannelore Ehrenreich. 


\subsubsection{Sensorymotor gating, sociability, conditional learning and working memory of miR-212/132 $2^{-/-}$mice}

Habituation to redundant sensory information is required to protect overloading of central nervous system with irrelevant stimuli ${ }^{298}$. Sensorimotor gating is the neurological process which filters out the irrelevant stimuli. Prepulse inhibition is the attenuation of reaction of an organism to a startling stimulus by the inhibitory effect of a prestimulus. Alteration in sensorimotor gating is associated with schizophrenia ${ }^{299}$. The prepulse inhibition in mice is tested by applying startle acoustical stimuli after a prepulse stimulus and measuring the startle response. In WT mice the inhibition of the startle response increases as the intensity of the prepulse stimulus increases. The same pattern was also seen in miR-212/132/- mice (Fig. 11a). The magnitude of the startle response is same for both genotypes, which rule out the possibility of auditory defects (Fig.11b). Therefore, these results suggest that the mechanisms of sensorimotor gating are not altered in the miR-212/132-/- mice.

For mice, recognition of conspecifics is important for maintaining social hierarchy and for mate choice. The social interaction test is used to measure the social affiliation and social memory of the mice, by measuring the time it spends with familiar and unfamiliar mice. The social index can be defined as the amount of time spent around a wire chamber where a stranger mouse is kept compared to the time spent to explore an empty chamber. The difference between the social indices of WT and miR-212/132/-- mice was statistically insignificant (Fig. 11c). The memory index, which shows the ability of mouse to distinguish the familiar mouse from unfamiliar mouse, was also similar in both genotypes (Fig. 11d). Therefore the sociability and ability to remember the acquaintance (social memory) is not altered in miR-212/132/- mice.

The fear-conditioning test is used to assess the ability of mice to learn and remember the association between an environmental cue and an aversive stimulus ${ }^{300}$. The "baseline" measurement was done by putting the mice into a novel context and recording the freezing response for $2 \mathrm{~min}$. Then a tone, which was also used as conditioned stimulus (CS), was applied and the percentage of freezing was recorded to determine the "basecue". To mediate the fear conditioning, CS and foot shock was delivered. The mouse exposed to the aversive experience was expected to learn and remember the association between the spatial cues of the chamber, the 
auditory stimulus and the foot shock. Two days later contextual memory was tested by putting these mice into the same chamber. They showed freezing behavior as a sign of fear, which is associated with the previously experienced foot shock. The miR-212/132-/- showed similar amount of freezing response (Fig. 11e). To measure the ability of mice to associate foot shock with the CS, they were put into a cage, which is different than the chamber and the CS was delivered. The percentage of freezing in WT mice was not different than miR-212/132/- mice (Fig. 11e) which indicates that miR-212/132 deficiency has no significant influence on conditional memory formation.

8-arm radial maze test measures the working memory (short-term memory) capabilities of mice. Water deprived mice were put into the middle of maze where there are 8 radial arms. The mice were expected to explore the arms and consume the liquid reward at the end of each arm in $15 \mathrm{~min}$. The same session was repeated for 11 times in consecutive days. Working memory is measured when the mice enter each arm a single time. Any error in the working memory would lead to re-entry into the arms. Visiting the already explored arms was recorded as a mistake. The number of mistakes done by WT animals decreased as the trial sessions progress. MiR-212/132 $2^{-/}$animals performed as good as their WT littermates (Fig. 11f). This data suggest that the short-term memory is not impaired due to lack of miR-212/132. 
A

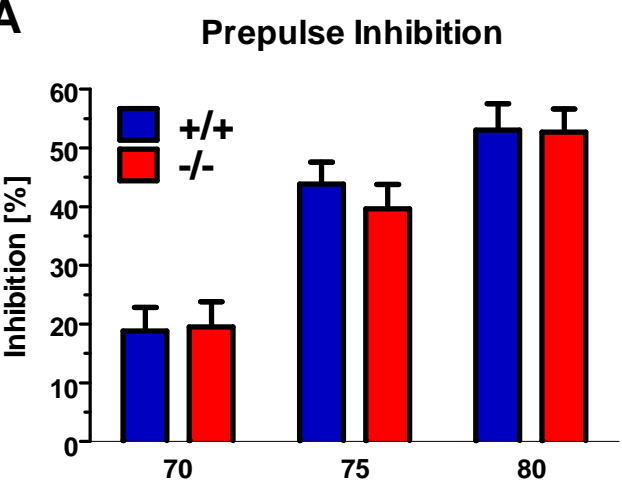

C
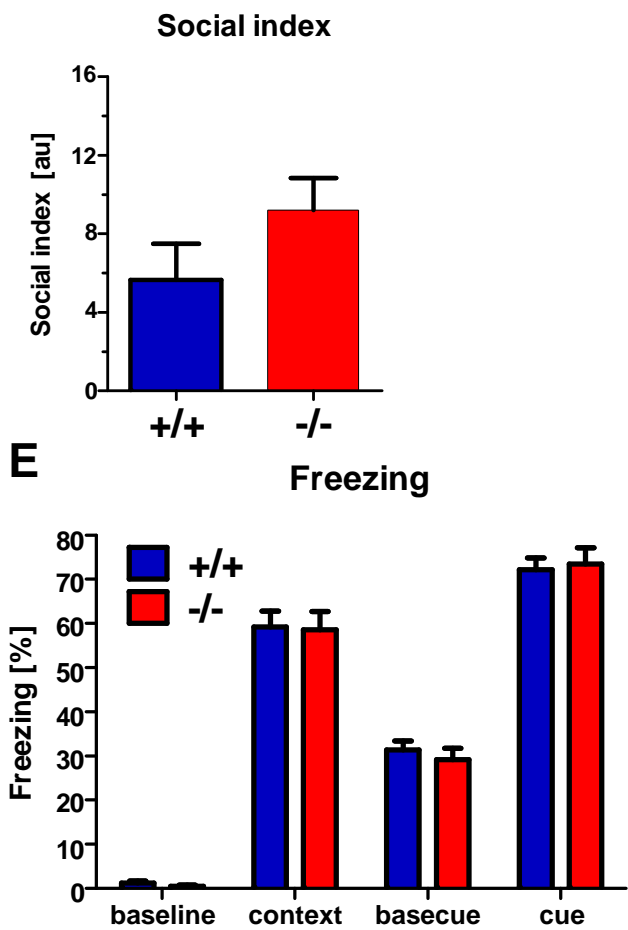

B

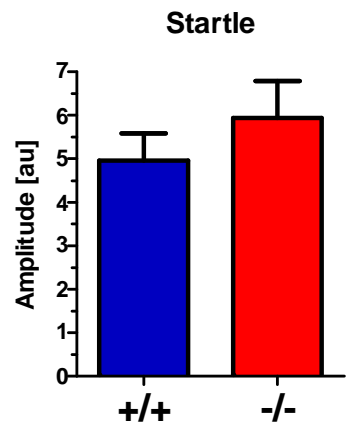

D

Memory index

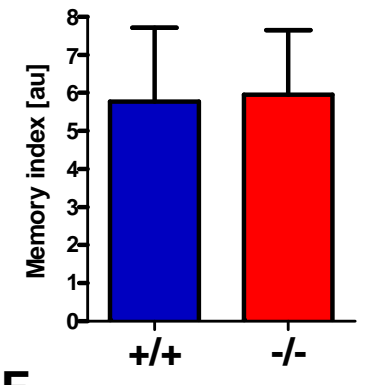

$\mathbf{F}$

8-arm maze

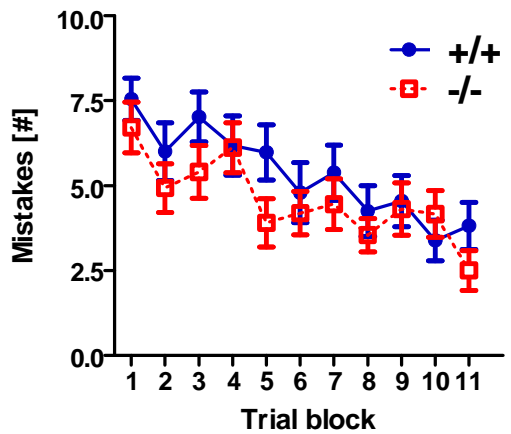

Figure 11: Sensorimotor gating, sociability, fear conditioning and 8-arm maze assays on WT and miR212/132-/- mice. (a, b) In the prepulse inhibition test, miR-212/132 ${ }^{-/}$mice demonstrated similar levels of inhibition (a) and startle amplitudes (b) as WT littermates. (c, d) In the tripartite chamber test, both social (c) and memory (d) indices of miR-212/132 $2^{-/-}$mice were similar to WT controls. (e) In cued and contextual fear conditioning tests, miR-212/132 ${ }^{-/}$mice displayed similar levels of freezing behavior compared to their WT littermates. (f) In 8-arm radial maze task, the number of mistakes done was similar between miR-212/132 and WT mice. These experiments were conducted by Dr. Konstantin Radyushkin in the research group of Prof. Dr. Dr. Hannelore Ehrenreich. 


\subsubsection{Activity levels and spatial memory of miR-212/132-/- mice}

The open field assay evaluates the behavior for the anxiety, motor function and exploratory behavior components ${ }^{301}$. It can be considered as a modified version of elevated plus maze. Mice are put into a circular arena and the time spent in different compartments of the arena and the total distances they have covered are recorded. As the mice explore the arena, they move to the intermediate and the central zones. However they prefer to spend most of their time at the periphery especially when they feel anxiety or fear. Additionally the total distance they cover gives clues about the locomotor activity of mice. Interestingly the miR-212/132 ${ }^{-/}$mice covered slightly but significantly more distance than WT mice in the open field arena (Fig. 12a). However the time spent in different zones of the arena does not differ between the genotypes (Fig 12b). Therefore these results indicate that miR-212/132/- mice are slightly hyperactive than WT littermates but their anxiety levels are normal.

Morris water maze is an assay that assesses the learning and memory abilities of mice or rats $^{302}$. The mice are put into a pool divided into quadrants and filled with opaque or transparent water. A platform is located into one of the quadrants of the pool that mice can climb and escape the water. During the training sessions mice are expected to learn, memorize and then recall the location of the platform by considering the spatial cues. In normal situations, during the course of the trainings, escape latency of mice decreases, which is mainly because mice spends less time for seeking the platform but rather remembers the position and directly swims towards it. Initially visible platform test was performed to check if miR-212/132 $2^{-/}$mice have any defect in vision or motivation and ability to escape the water. The escape latencies of mice of either genotype did not differ (Fig. 12c). When the platform was hidden, the escape latencies of WT and miR-212/132/- mice decreased from $60 \mathrm{sec}$ to approximately $40 \mathrm{sec}$ in 3 days (Fig. 12d). The escape latency of WT mice continued to decrease following the $3^{\text {rd }}$ day in the trial and reached to a plateau phase at $7^{\text {th }}$ day. In contrast miR-212/132-/- mice could not develop their performance after the $3^{\text {rd }}$ day and the escape latency stayed the same till the end of the training sessions (Fig. 12d). The platform was removed to conduct a probe trial. The time spent in the target quadrant (where the platform was located) was longer for WT mice compared to miR$212 / 132^{-/-}$mice. The representative trajectory of mice shows that WT mice were making focal 
search for the platform at the target quadrant. However miR-212/132 $2^{-/}$mice were most likely using nonspatial, chaining strategy (Fig. 12e). During the first 2-3 days of the trial, mice use nonspatial strategies like thigmotaxic, random or chain swimming. Later on they develop spatial strategies, which substantially reduces the escape latency ${ }^{303,304}$. MiR-212/132/- mice perform worse than their WT littermates in navigating towards the platform in subsequent days after the $3^{\text {rd }}$ day of the trials (Fig. 12d). Therefore these results demonstrate that the spatial-memory related cognitive capabilities are impaired in the miR-212/132/- mice. The swimming velocity of WT and miR-212/132/- were similar (Fig. 12f). Together with the results in the visible platform test, this finding rules out the possibility that the performance of miR-212/132/- mice is influenced by other alterations in sensory or motor functions or the motivation and ability to escape the water. 
A

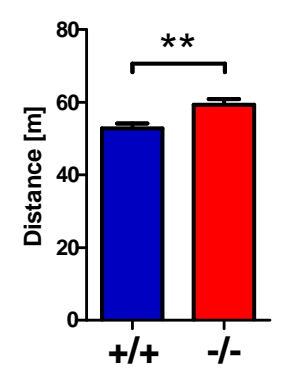

C

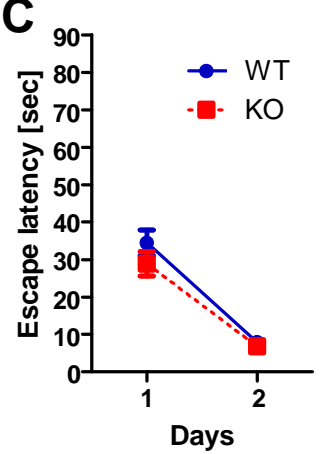

E

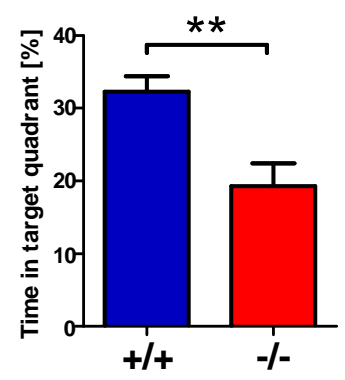

B

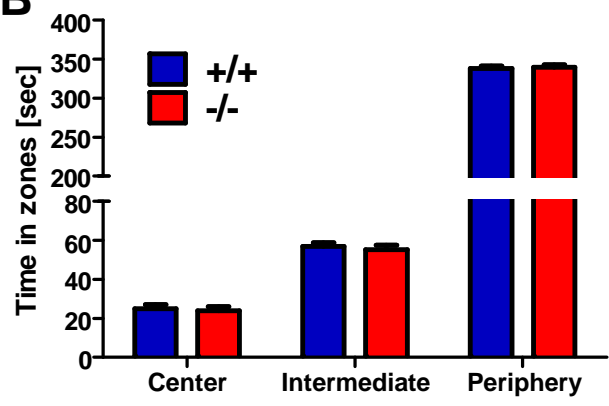

D

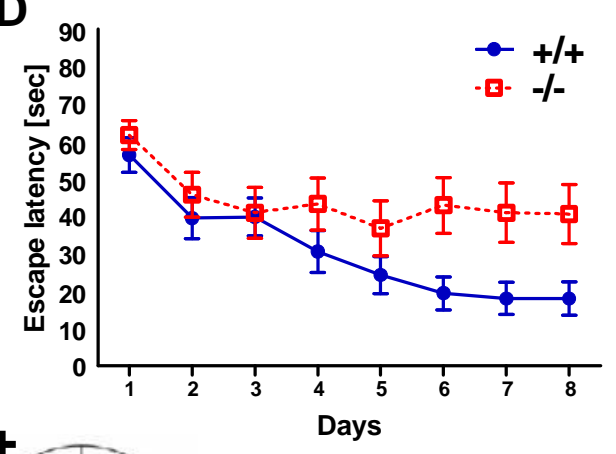

$\mathbf{F}$

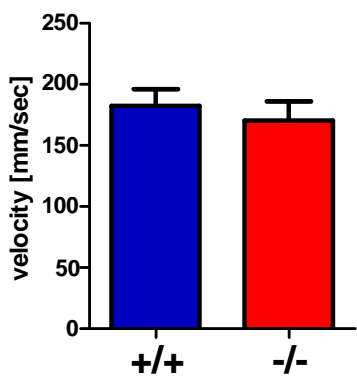

Figure 12: Open field and Morris water maze assays on WT and miR-212/132 $2^{-/-}$mice. (a,b) In the open field test, the total distance traveled within $7 \mathrm{~min}$ period was higher for $\mathrm{miR}-212 / 132^{-/-}$compared to WT littermates (a), but the time spent in different zones were similar (b), indicating hyperactivity but no anxiety in miR-212/132 ${ }^{-/}$mice. (Mann-Whitney U-test, **: $\mathbf{p}<\mathbf{0 . 0 0 6 2}, \mathbf{n = 2 2 - 2 3 . )}(\mathrm{c}-\mathrm{f})$ In the Morris water maze test, the learning performances of $\mathrm{miR}-212 / 132^{-/-}$mice were similar to WT littermates in visible platform version (c), but significantly worse in the hidden platform version of the test (2-way ANOVA for repeated measures for genotype versus trials interaction $F_{(7,266)}=13.3$, $p<0.0001, n=22-23$;). In the hidden platform version, the time spent in the target quadrant was less for miR-212/132 ${ }^{-/}$mice (Mann-Whitney U-test, *: $\mathrm{p}<0.003, \mathrm{n}=22$ 23) although the average velocity of $\mathrm{miR}-212 / 132^{-/-}$mice was similar to WT mice (f). Representative swimming trajectories of WT and miR-212/132 $2^{-/-}$mice are shown in (e). The place of the hidden platform is shown with a circle within the upper left target quadrant. These experiments were conducted by Dr. Konstantin Radyushkin in the research group of Prof. Dr. Dr. Hannelore Ehrenreich. 


\subsection{Electrophysiological characterization of miR-212/132/- mice}

Electrophysiology is the study of the electrical properties of single cells or tissues. It measures the electrical voltage or current change from single ion channels or even tissues. Thus electrical activity or action potential activity of neurons located in the hippocampus can be recorded.

High expression of miR-212/132 in different hippocampal regions and observation of impaired spatial memory in miR-212/132/- mice suggests functional role of the gene in hippocampal networks. Since miR-212/132 expression in CA3 and CA1 region was observed, electrophysiological analyses on Schaffer collateral pathway was performed to assess the functional role of miR-212/132 in hippocampal networking.

\subsection{1. $\operatorname{miR}-212 / 132$ increases the synaptic facilitation without affecting the synaptic transmission}

Invading action potential mediates the opening of calcium channels at presynaptic compartment where $\mathrm{Ca}^{2+}$ ions influx through the channels. The $\mathrm{Ca}^{2+}$ concentration declines to basal levels in couple of hundred milliseconds. However if second action potential arrives in a shorter time to the same compartment, more $\mathrm{Ca}^{2+}$ is added up to the residual $\mathrm{Ca}^{2+}$, which in turn increases the neurotransmitter release probability. This short-term synaptic plasticity phenomenon is called paired pulse facilitation $(\mathrm{PPF})^{305,306}$. Paired pulse ratio (PPR) protocol applied on Schaffer collateral pathway of acute hippocampal slices obtained from 4-5 weeks old WT and miR-212/132 ${ }^{-/-}$mice revealed that there is a significant increase in the PPR of miR212/132/- animals compared to their WT littermates as well (Fig. 13a). This data demonstrates increase in short-term synaptic facilitation in miR-212/132/- Schaffer collateral-CA1 synapses. To check if the difference in short-term synaptic facilitation is due to any alteration in basal excitatory synaptic transmission, the input-output relationship of Schaffer collateral inputs to postsynaptic CA1 neurons was measured. However no difference between mutant and WT samples was detected (Fig. 13b), suggesting that the synaptic transmission is not impaired in miR-212/132 mutant mice. 

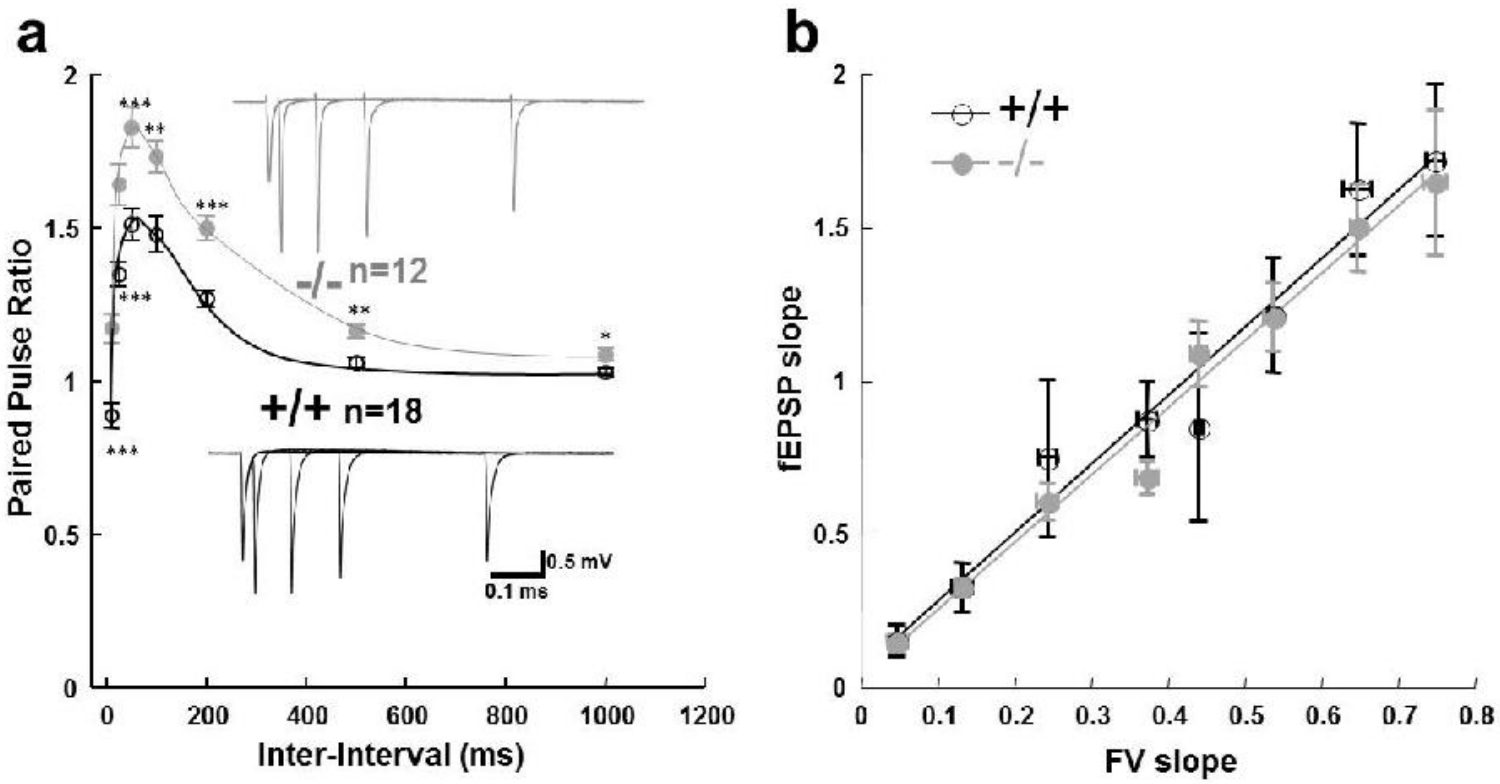

Figure 13: (a) Paired-Pulse Ratios (PPRs) are higher in miR-212/132 ${ }^{-/}$mice hippocampal slices compared to WT controls. (Unpaired t-test, two-tailed, *:p<0.05, **:p<0.01, ***:p<0.001) The number of slices (n) analyzed are depicted on the graphs. Representative fEPSP traces for both experiments are shown above the corresponding graphs. (b) Input-output curve of field excitatory postsynaptic potential (fEPSP) at Schaffer collateral-CA1 synapses is similar in both miR-212/132 $2^{-/-}$and WT hippocampal slices. These experiments were conducted by Dr. JeongSeop Rhee.

\subsection{2. $\operatorname{miR}-212 / 132$ is essential for proper regulation of $\mathrm{L}$ - LTP but not for E-LTP}

Long-term potentiation (LTP) is the persistent increase in the size of excitatory postsynaptic current (EPSC), EPSP or field EPSP (fEPSP). The increase in synaptic response can last for hours. LTP is considered as cellular mechanism, which composes the fundamentals of learning and memory dependent plasticity in the brain. LTP has two forms; early-LTP (E-LTP) and lateLTP (L-LTP), which are protein-kinase dependent and protein synthesis dependent respectively. E-LTP lasts for 1-2 hours while L-LTP can stay stable as long as the hippocampal slice is alive $^{307}$. High frequency stimulation (HFS) was applied on Schaffer collateral axons to induce LTP in miR-212/132 $2^{-/}$and WT acute hippocampal slices. E-LTP was not affected in miR212/132 $2^{-/}$Schaffer collateral pathway (Fig. 14a), however L-LTP was significantly enhanced for 2 hours following the induction compared to WT counterparts (Fig.14b). These results suggest that miR-212/132 is involved in the regulation of maintenance of LTP rather than initiation of it. 
a

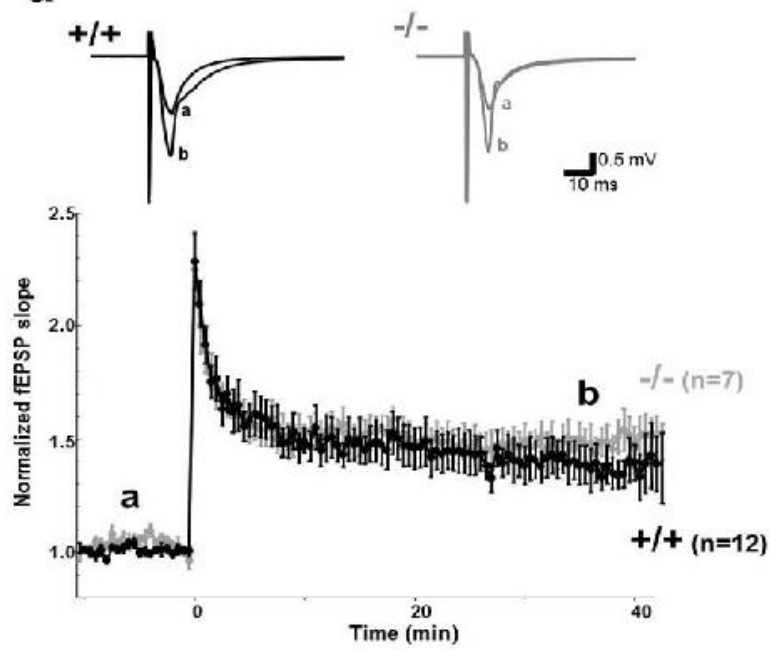

b

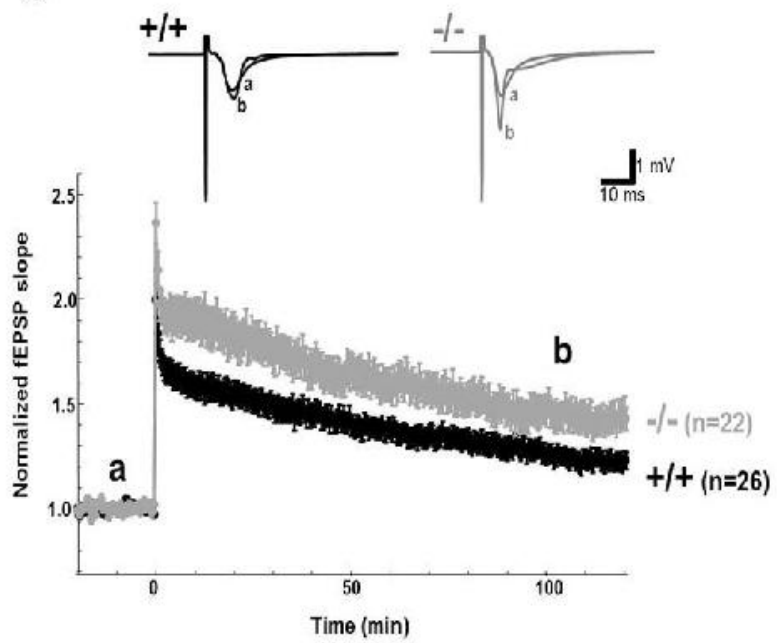

Figure 14: Early-phase LTP (E-LTP) and late-phase LTP (L-LTP) on WT and miR-212/132/- hippocampal slices. (a) E-LTP was similar in miR-212/132 ${ }^{-/-}$and WT hippocampal slices, whereas (b) late-phase LTP was significantly enhanced in miR-212/132 ${ }^{-/}$slices compared to WT slices. The number of slices (n) analyzed are depicted on the graphs. (Unpaired t-test, two tailed, at analyzed time points: $0.001<$ p $<0.01$ ). These experiments were conducted by Dr. JeongSeop Rhee.

\subsection{3. miR-212/132 does not have significant influence on LTD and depotentiation}

Long-term depression (LTD) is the mirror image of LTP. It is long-lasting and activitydependent decrease in synaptic potentiation. LTD is a component of synaptic plasticity, which is essential for the establishment of new neuronal networks to encode information ${ }^{307}$. Low frequency stimulation (LFS) was applied on Schaffer collaterals of miR-212/132/- and WT hippocampal slices. LTD was induced at the same rates in both genotypes (Fig 15), which suggest that miR-212/132 deficiency does not impair the regulation of LTD. 


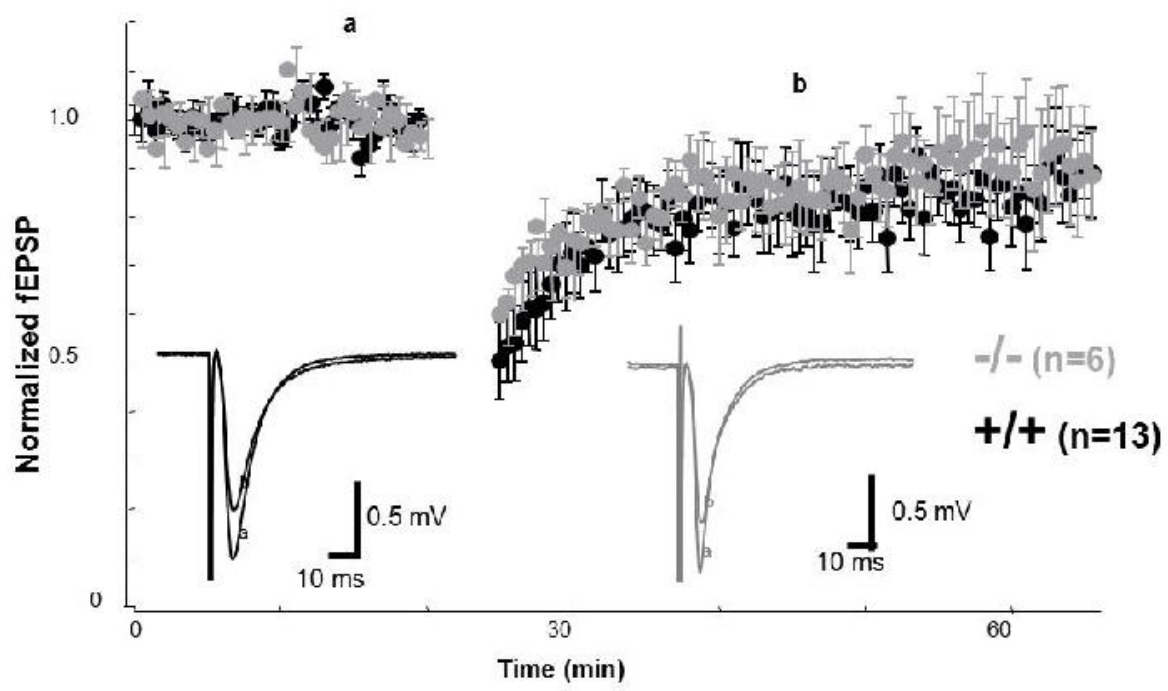

Figure 15: LTD on miR-212/132 ${ }^{-/}$and WT hippocampal slices. LFS application (1 $\mathrm{Hz}$ for 15 min) showed that NMDAR-dependent LTD levels were not altered in miR-212/132 ${ }^{-/-}$hippocampal slices. The number of slices (n) analyzed are depicted on the graphs. This experiment was conducted by Dr. JeongSeop Rhee.

Depotentiation is the reversal of LTP, which means weakening of synapse that was already potentiated. In a previous study, it was speculated that miR-212/132 expression is mGluR dependent, thus functionally it is correlated with depotentiation rather than LTP ${ }^{126}$. Therefore the depotentiation capacity on hippocampal slices was studied to check if the absence of miR-212/132 alters the depotentiation in Schaffer collateral-CA1 system. LFS was applied 2 min and 40 min following the application of HFS. The depotentiation at Schaffer collateral-CA1 synapses of miR-212/132 $2^{-/-}$mice was not different than their WT littermates (Fig. 16a, b). Taken together, miR-212/132 dependent deficiency in synaptic plasticity leads to higher PPF and LLTP but does not influence E-LTP, LTD and depotentiation. 

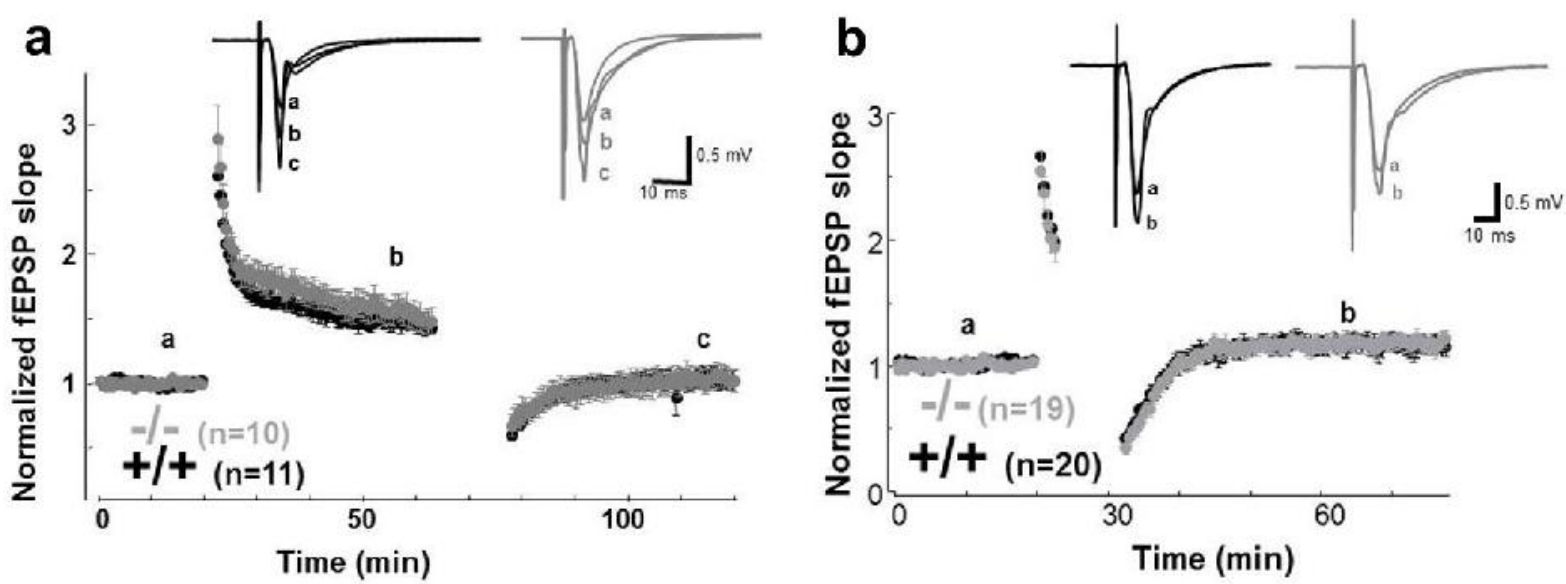

Figure 16: Depotentiation experiment on miR-212/132/- and WT hippocampal slices. (a,b) Induction of the depotentiation $40 \mathrm{~min}$ (a) or $2 \mathrm{~min}$ (b) after the HFS showed no difference in depotentiation capacity of miR212/132 ${ }^{-/-}$hippocampal slices compared to WT slices. 2 trains of $100 \mathrm{~Hz}$ for 1 sec were applied as HFS. $2 \mathrm{~Hz}$ for $10 \mathrm{~min}$ or $1 \mathrm{~Hz}$ for $15 \mathrm{~min}$ was applied as LFS. The number of slices (n) analyzed are depicted on the graphs. Representative fEPSP traces for all experiments are shown above the corresponding graphs. These experiments were conducted by Dr. JeongSeop Rhee.

\subsection{The molecular functions of miR-212/132 in hippocampal neurons}

Electrophysiological assays can be used to explain the mechanism of the observed impaired spatial memory and hyperactivity of miR-212/132-/- mice at the cellular level. However, to have a deeper insight into the molecular mechanism of the miR-212/132 function, it is necessary to characterize the molecular functions and interactions of miR-212/132.

\subsubsection{Primary hippocampal neuron cultures as experimental setup}

MecP2, p250GAP and SIRT1 were shown to be the targets of miR-212/132 in several studies (Table 1). To confirm if they are indeed targeted by miR-212/132 in the murine hippocampus the levels of MecP2, p250GAP and SIRT1 were checked in total protein lysates obtained from whole hippocampi of age-matched miR-212/132 ${ }^{-/-}$and WT mice (Fig. 17a; each 
lane represents an individual mouse hippocampus). MecP2 and p250GAP levels were not different in total hippocampal lysates obtained from mice of either genotype. SIRT1 levels tend to be higher in miR-212/132/- hippocampi, although the difference did not reach to statistically significant levels (Fig. 17a,b).

The expression of miR-212/132 is spatially restricted in specific regions of the mouse hippocampus (Fig 8,9) and expressed by a relatively minor population of cells within the hippocampus. When total protein is isolated from the whole hippocampus, the impact of the loss of miR-212/132 might have been underestimated due to the dilution of the proteome of miR212/132-expressing cells by the proteome of the miR-212/132 negative cells. This dilution may bring the threshold of detection of biologically relevant differences far below the sensitivity of western blotting. Moreover, there is a high variation in the levels of MecP2, SIRT1 and p250GAP (Fig. 17a) among individual samples. Although mice used in these experiments were selected as being littermates and being housed in the same cage, the levels of proteins in the hippocampus of each individual mouse might be different at the time of dissection. In other words the heterogeneity of the system might be the explanation underlying the observed inconsistent data with the findings in the literature.

To be able to decrease the heterogeneity-related variations between samples I decided to use the primary hippocampal neuron cultures. In this regard it can be assumed that each cultured cell will be exposed to the same culture conditions and drug applications will affect all the cells in the same manner, which subsequently may decrease the dilution effect and the heterogeneityrelated variations. 


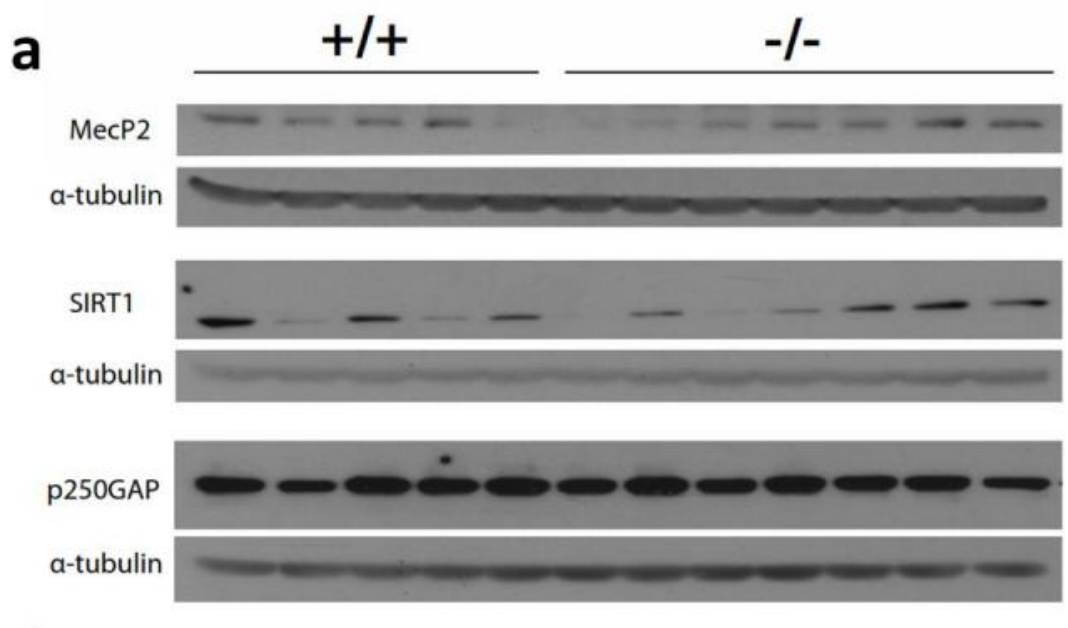

b

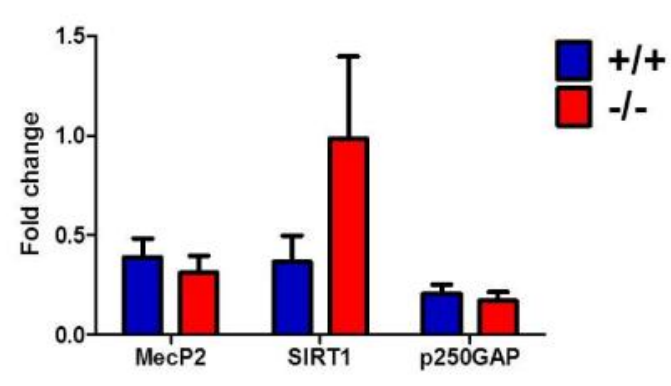

Figure 17: MecP2, SIRT1 and p250GAP levels in WT and miR-212/132-/ mice hippocampus. (a) Approximately 2 months old male mice hippocampi were dissected and the total protein was isolated. Equal concentration of protein was loaded into each lane. Every lane represents an individual mouse sample. (b) The densitometric analysis was performed by Fiji software. $\alpha$-tubulin was used as endogenous control. Unpaired t-test, two-tailed, $\mathrm{p}>0.05,+/+: \mathrm{n}=5 ;-/-: \mathrm{n}=7$.

\subsubsection{Induction of mature miR-212/132 production in hippocampal neuron cultures}

Neuronal activity regulates miR-212/132 gene expression (as explained in section 1.2.1). To check if miR-212/132 expression can be enhanced by pharmaceutical induction of neuronal activity in hippocampal neuron cultures, kainic acid was administered for 6 hrs. Kainic acid is a kainate receptor agonist, which mediates robust neuronal activation ${ }^{308-310}$. It was also shown to induce miR-132 expression in mouse hippocampus ${ }^{127}$. After 6 hours of incubation, total RNA was isolated from neurons and mature miRNA specific RT Q-PCR was performed. The levels of miR-212 and miR-132 were induced by 2 and 1.7 folds respectively in kainic acid treated neurons as compared to untreated neurons (Fig. 18a, b). This result demonstrates that the biogenesis of mature miR-212 and miR-132 can be upregulated by inducing neuronal activity in 
this experimental setup, which allows me to study the alterations in the mutant hippocampal neurons under miR-212/132 inducing conditions.

a

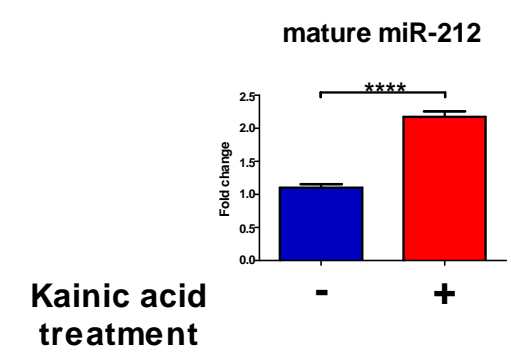

b

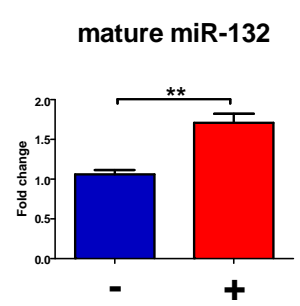

C

mature miR-134

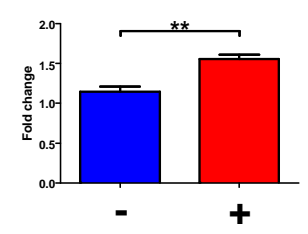

Figure 18: Increase in miR-212, -132 and -134 levels. 30 M kainic acid treatment for 6 hrs upregulated mature miR-212 (a), miR-132 (b) and miR-134 (c) production. Paired t-test, two-tailed p-value, ${ }^{* *}:<0.01$, *****: $<0.0001, \mathrm{n}=6$.

\subsubsection{Impaired immediate early gene regulation}

In contrast to the short-term memory, the long-term memory is transcription and translation dependent ${ }^{311}$. If protein synthesis inhibitors are administered to an animal just after learning process, the long-term memory storage can be prevented. However if the administration is done several hours after the learning, then long-term memory cannot be blocked. The same phenomenon is valid for $\mathrm{LTP}^{312}$, which is considered as the model of learning. Therefore, the gene expression, which is induced just after neuronal activity, is required to encode and store memories. The group of neuronal activity induced genes is called immediate early genes (IEG). Those genes mediate the neuronal activity dependent remodeling of synapses ${ }^{313}$. Since miR$212 / 132^{-/-}$mice show improper LTP, there might be a possible defect in the regulation of Arc, cFos and BDNF in kainic acid treated miR-212/132/- hippocampal neurons.

C-Fos induction and relative transcript levels were checked in kainic acid treated and untreated WT and miR-212/132/- hippocampal neurons. The induction of neuronal activity in hippocampal neurons by 30 min of kainic acid application was sufficient to obtain upregulated levels of c-Fos transcript in the WT neurons. The c-Fos levels increased further till 3 hours and subsequently decreased after $6 \mathrm{hrs}$ of incubation. The levels of c-Fos in miR-212/132/- neurons 
showed the same trend of increase in $30 \mathrm{~min}$ and $3 \mathrm{hrs}$ and then decreased after $6 \mathrm{hrs}$. Interestingly the upregulation levels were significantly higher in miR-212/132-- neurons at each time point compared to WT neurons (Fig. 19). The level of c-Fos was significantly lower in untreated miR-212/132 $2^{-/}$neurons, compared to WT neurons (Fig 22a). The relative transcript amounts were at the same levels at $30 \mathrm{~min}$ and $3 \mathrm{hrs}$ time points (Fig. 22b,c). However, there were 1.5 fold more c-Fos transcripts in miR-212/132/- neurons compared to WT neurons after 6 hours of incubation with kainic acid (Fig. 22d). These results suggest that although the basal levels of c-Fos is lower in miR-212/132/- neurons, observed increase in fold induction compensates the c-Fos levels in $30 \mathrm{~min}$ and $3 \mathrm{hrs}$ of kainic acid application. However, since the c-Fos transcription obviously cannot be suppressed properly, this leads to higher c-Fos transcript levels at the time point of $6 \mathrm{hrs}$.

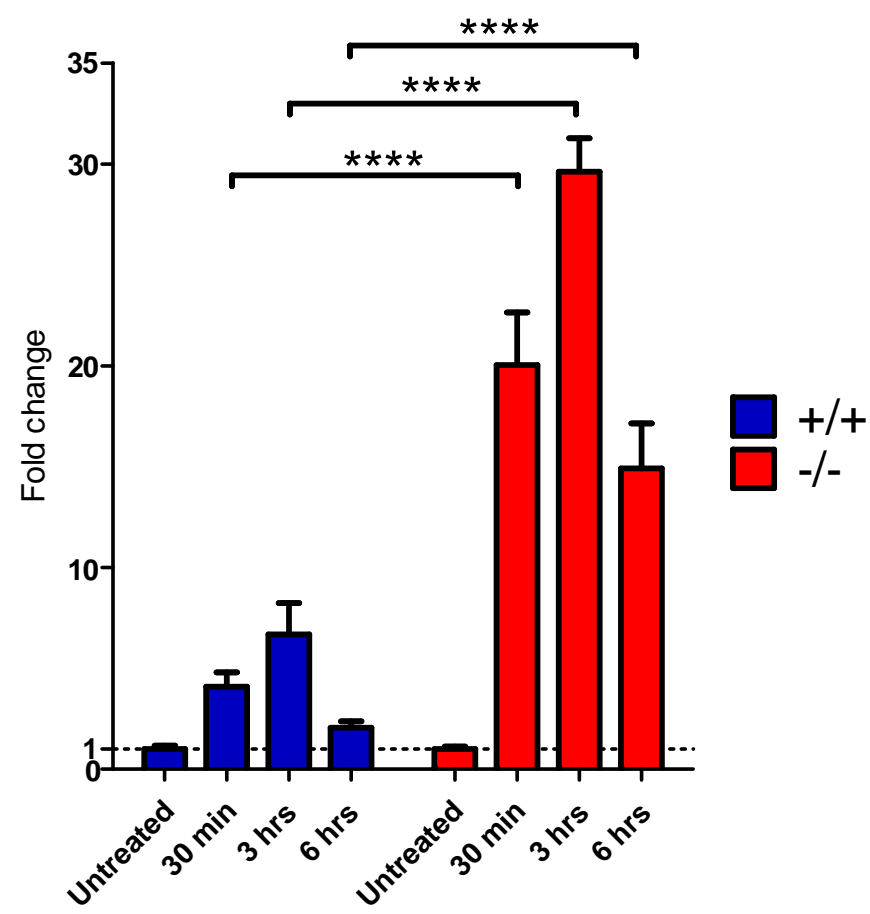

Kainic acid treatment

Figure 19: C-Fos transcript levels in cultured hippocampal neurons. $30 \mathrm{M}$ kainic acid treatment was performed for indicated amount of time. Relative fold changes were calculated by comparing the time points to the untreated group of the same genotype. Bonferroni multiple comparison test, ****: p<0.0001. 2-way ANOVA for genotype versus treatment interaction $\mathrm{F}_{(3,23)}=\mathbf{2 0 . 3 4}, \mathbf{p}<\mathbf{0 . 0 0 0 1}$; for difference between genotypes $F_{(1,23)}=149.76, p<0.0001$; for difference over treatment time $F_{(3,23)}=44.77, p<0.0001$, untreated; $n=4,30 \mathrm{~min}$; $\mathrm{n}=4,3 \mathrm{hrs} ; \mathrm{n}=3$, 6 hrs; $\mathrm{n}=4$ 
The levels of Arc, which is an important regulator of LTP maintenance and long-term memory, was therefore checked in WT and miR-212/132-/- hippocampal neurons. Similar to cFos, the transcript levels of Arc were also lower in untreated miR-212/132/- hippocampal neurons compared to the control group (Fig. 22a). Treatment with kainic acid for $30 \mathrm{~min}$ enhanced the Arc level in WT neurons as well as in miR-212/132/- neurons. However the fold induction in WT neurons was approximately 2 fold while the induction was by 10 fold in miR$212 / 132^{-/-}$neurons. The fold differences of Arc at $3 \mathrm{hrs}$ and $6 \mathrm{hrs}$ time point group relative to levels in the corresponding untreated groups were higher in miR-212/132/- neurons compared to the WT neurons as well (Fig. 20). Although the basal level of Arc was lower in miR-212/132-/neurons, the levels were not different at $30 \mathrm{~min}$ and $3 \mathrm{hrs}$ time points (Fig. 22b, c). However, approximately 2 fold more Arc transcripts were observed in miR-212/132/- hippocampal neurons after 6 hrs of kainic acid application (Fig. 22d). Lower basal levels of Arc in miR-212/132/neurons was compensated after $30 \mathrm{~min}$ and $3 \mathrm{hrs}$ and then its levels got even higher after $6 \mathrm{hrs}$ of kainic acid treatment compared to WT neurons. The alteration in the regulation of induction and suppression of the Arc might be the leading cause of observed differences in fold changes and relative levels. 


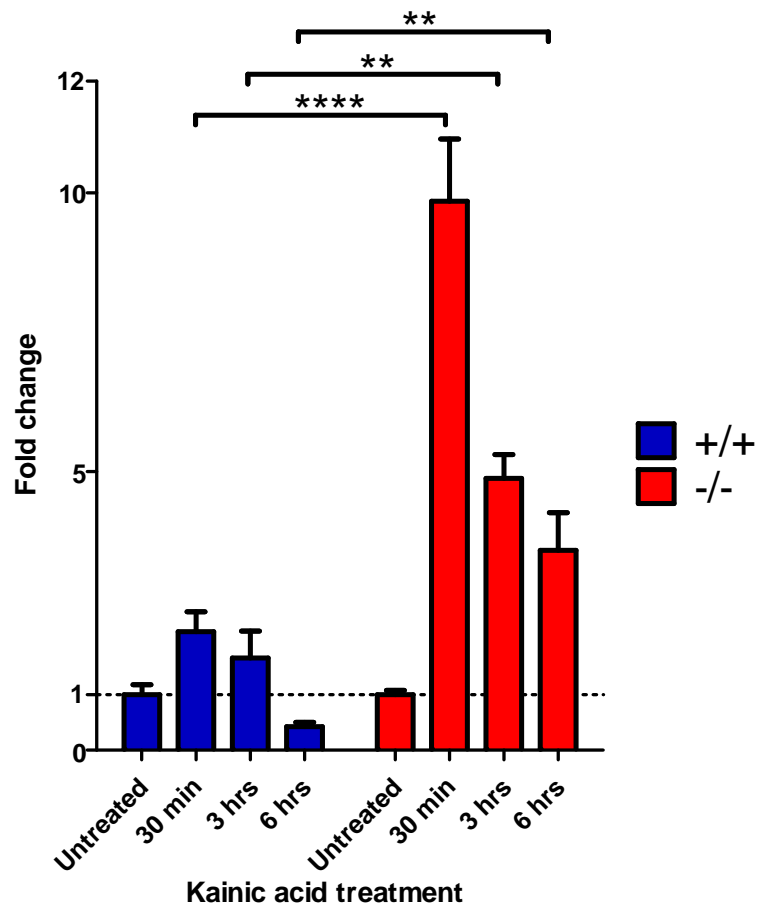

Figure 20: Arc transcript levels in cultured hippocampal neurons. 30 M kainic acid treatment was performed for indicated amount of time. Relative fold changes were calculated by comparing the time points to the untreated group of the same genotype. Bonferroni multiple comparison test, $* * * *: \mathbf{p}<0.0001, * *: p<0.01$. 2-way ANOVA for genotype versus treatment interaction $F_{(3,23)}=17.8, p<0.0001$; for difference between genotypes $F_{(1,23)}=84.31$, $p<0.0001$; for difference over treatment time $F_{(3,23)}=32.86, p<0.0001$, untreated; $n=4$, 30 min; $n=4,3 h r s ; n=3,6$ hrs; $n=4$.

Previous studies showed an association between the regulation of BDNF and miR$212 / 132^{125,128,219,314,315}$. Therefore, I checked the regulation of BDNF expression in WT and miR$212 / 132^{-/-}$hippocampal neurons at different time points after kainic acid induced neuronal activation. Unlike c-Fos and BDNF, 30 min of kainic acid treatment was not sufficient to observe the induction of BDNF expression (Fig. 21). However after 3 hours of kainic acid treatment, the BDNF levels were induced by approximately 1.5 fold in WT and 2.5 fold in miR$212 / 132^{-/-}$neurons (Fig 21). The fold increase after 6 hrs of kainic acid treatment was higher in miR-212/132-/- neurons compared to WT neurons as well (Fig 21). The level of BDNF transcripts in untreated and 30 min kainic acid treated miR-212/132/- neurons was lower than WT neurons (Fig. 22a, b). No difference in the levels were observed at 3 hrs and 6 hrs treatment time points (Fig. 22c, d). These results indicate that the loss of miR-212/132 function leads to 
downregulated BDNF levels in hippocampal neurons and alteration in the regulation of BDNF expression which is then reflected as high fold induction upon kainic acid treatment.

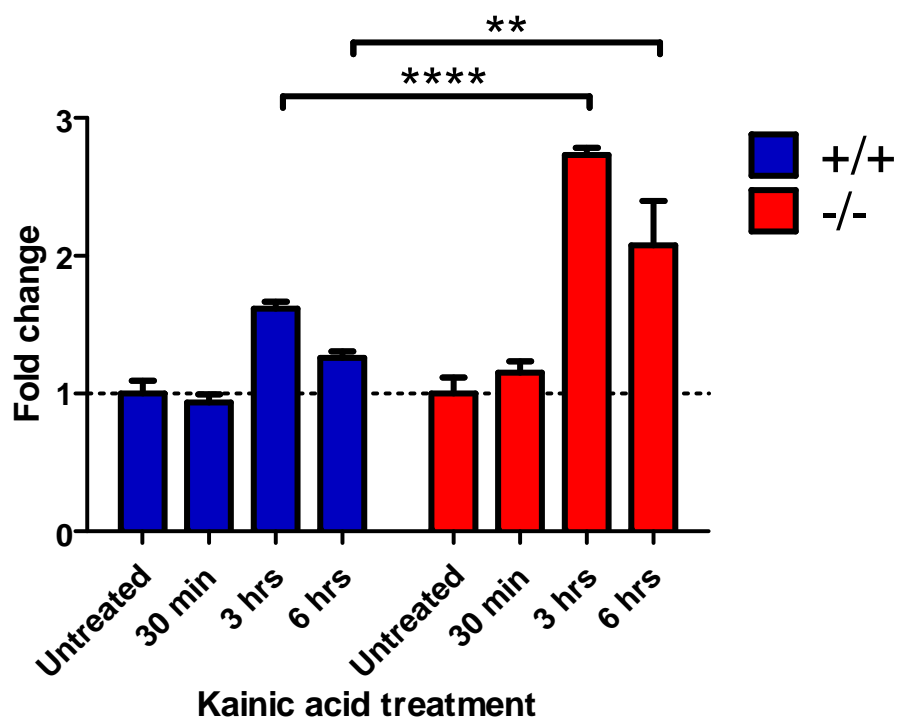

Figure 21: BDNF transcript levels in cultured hippocampal neurons. $30 \mathrm{M}$ kainic acid treatment was performed for indicated amount of time. Relative fold changes were calculated by comparing the time points to the untreated group of the same genotype. Bonferroni multiple comparison test, $* * * *: \mathbf{p}<0.0001, * *: \mathbf{p}<0.01$. 2-way ANOVA for genotype versus treatment interaction $F_{(3,23)}=6.8, p=0.0018$; for difference between genotypes $F_{(1,23)}=30.06$, $p<0.0001$; for difference over treatment time $F_{(3,23)}=31.24, p<0.0001$, untreated; $n=4$, 30 min; $n=4,3$ hrs; $n=3,6$ hrs; $n=4$

The loss-of-function of miR-212/132 results in the downregulation of c-Fos, Arc and BDNF in untreated hippocampal neurons. Interestingly, kainic acid treatment mediates stronger gene induction in miR-212/132/- neurons and the absolute levels of c-Fos and Arc in miR$212 / 132^{-/-}$neurons exceeds the levels in WT after $6 \mathrm{hrs}$. These results indicate that the regulation of c-Fos, Arc and BDNF expression is impaired in the absence of miR-212/132 function. 
a

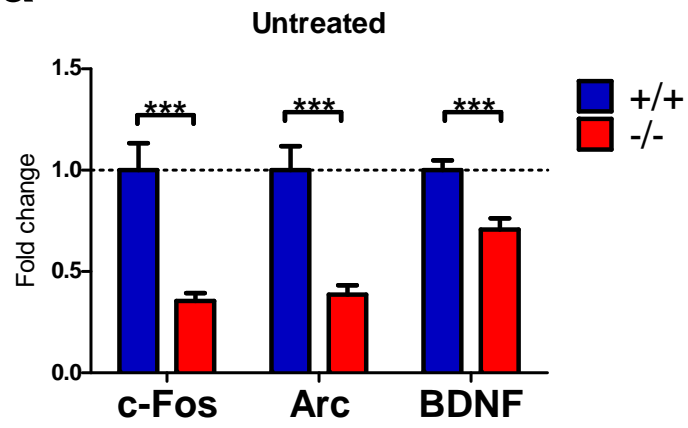

C

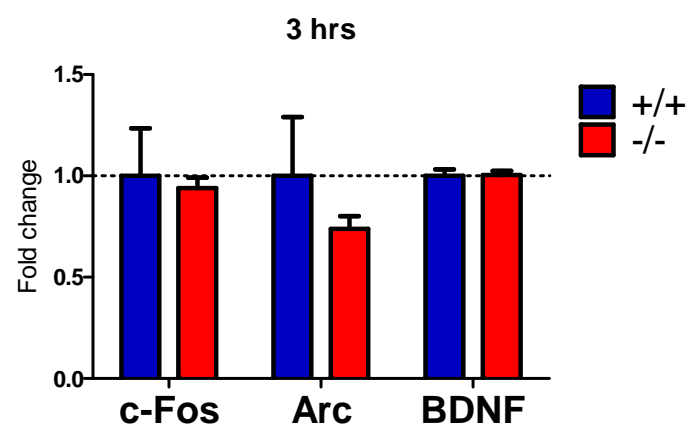

b
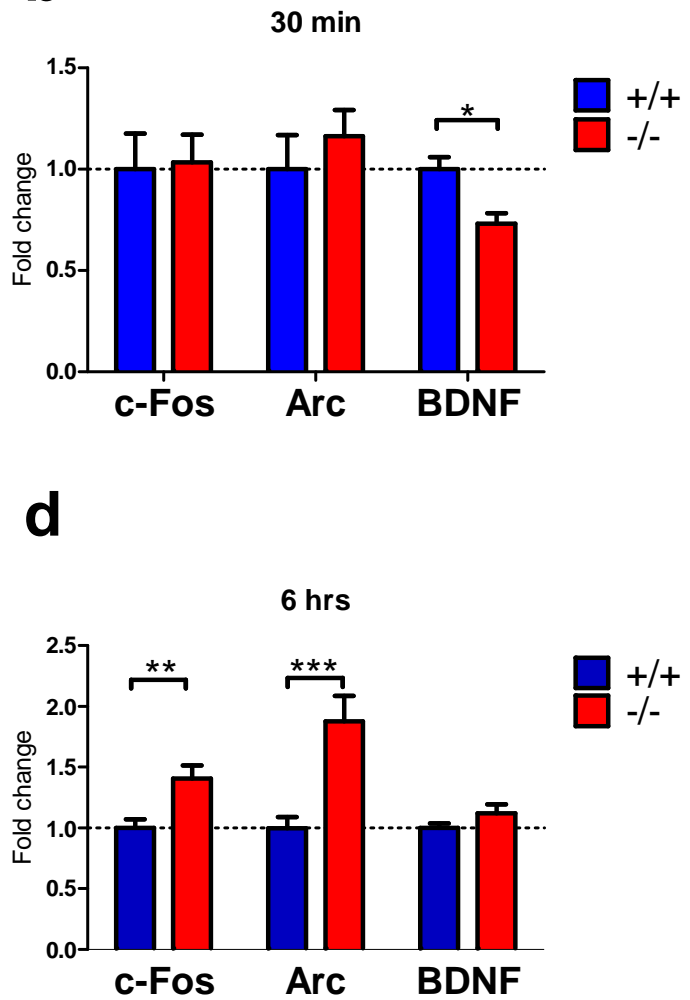

Figure 22: Relative transcript levels at different time points of kainic acid treatment. $30 \mathrm{M}$ of kainic acid treatment was performed for (b) $30 \mathrm{~min}$, (c) $3 \mathrm{hrs,} \mathrm{(d)} \mathrm{6hrs.} \mathrm{(a)} \mathrm{No} \mathrm{treatment} \mathrm{was} \mathrm{applied} \mathrm{on} \mathrm{the} \mathrm{control}$ group. The transcript levels of $+/+$ groups were set to 1 and relative transcript levels in $-/-$ groups were calculated. Unpaired t-test, two tailed, *: p<0.05, **: $\mathrm{p}<0.01, * * *: p<0.001$, ntreated; $n=10,30 \mathrm{~min} ; \mathrm{n}=4,3 \mathrm{hrs}$; $\mathrm{n}=3,6 \mathrm{hrs} ; \mathrm{n}=9$.

\subsubsection{Influence of miR-212/132 on SIRT1-miR-134-CREB pathway}

SIRT1 is the mammalian homolog of the NAD-dependent deacetylase Silent Information Regulator (Sir2) present in S.cerevisia, C. elegans and Drosophila. It has been known to be involved in ageing, ageing related disorders, metabolism control, oxidative stress regulation, circadian rhythms and cocaine addiction ${ }^{316-322}$. Recently it was shown that SIRT1 represses the expression of miR-134. One of the targets of miR-134 is cyclic AMP response element binding 
protein (CREB), which is a transcription factor. CREB has crucial function in linking the neuronal activity to gene expression. Membrane depolarization that drives influx of $\mathrm{Ca}^{2+}$ through ligand- and voltage-gated ion channels mediates the phosphorylation of CREB, which in turn activates its target genes by binding to the cAMP response element (CRE) in the regulatory sequences $^{313,323}$. SIRT1 deficiency causes upregulation of miR-134, which in turn downregulates CREB and BDNF in mouse hippocampus and consequently impairs synaptic plasticity and memory formation ${ }^{324}$.

SIRT1 is targeted by miR-132 $2^{146}$. Longer lifespan of miR-212/132/- mice (Fig. 4a) might therefore be associated with misregulation of SIRT1 in the absence of miR-132. Data presented in Fig. 22a shows that BDNF is downregulated in cultured primary hippocampal neurons. Moreover miR-212 and miR-132 are known as CREB regulated miRNAs ${ }^{116}$. Therefore, I hypothesized that miR-212/132 family might be involved in the SIRT1-miR-134-CREB pathway, which would then link the SIRT1 and CREB, thus creating a feedback loop (Fig. 23). Based on this hypothesis, it could be expected that in miR-212/132/- hippocampal neurons, SIRT1 levels would be higher, which in turn would downregulate miR-134 levels. Since miR134 targets $\mathrm{CREB}^{325}$, lower miR-134 levels might then lead to higher CREB levels. Upon induction of neuronal activity, higher CREB levels might accordingly be reflected as increased levels of phosphorylated CREB (pCREB). This putative molecular model may explain the observed increased fold induction of CREB regulated IEGs in miR-212/132/- hippocampal neurons as well (Fig. 19-21). According to this model there might be a feedback loop by which CREB and miR-212/132 family would regulate each other's' expression levels.

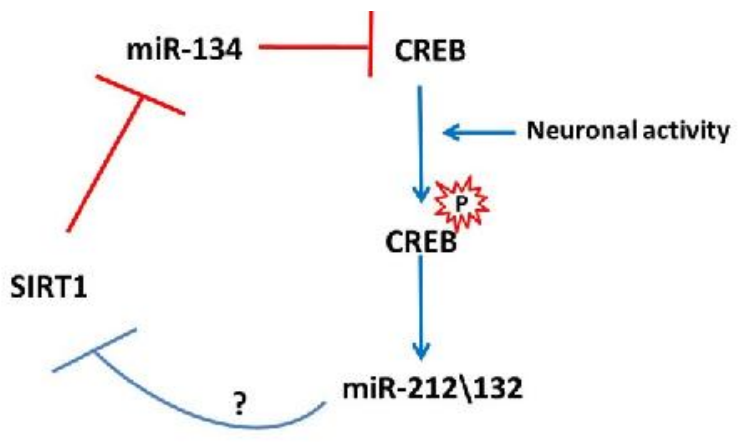

Figure 23: Proposed molecular model. SIRT1 represses miR-134. CREB is downregulated by miR-134 ${ }^{325}$. The phosphorylation of CREB is driven by neuronal activity ${ }^{313,323}$. Neuronal activity regulated miR-212/132 expression is induced by pCREB (refer to section 1.2.1). MiR-212/132 would be the link between CREB and SIRT1, which constitute a feedback loop. 
To validate this hypothesis I first asked if miR-134 is an activity regulated miRNA similar as miR-212 and miR-132. Six hours of kainic acid induction enhanced its expression level approximately 50\% (Fig. 18c) which suggests that miR-134 is an activity regulated gene and the kainic acid treatment on hippocampal neuron cultures provides appropriate conditions to mediate the miR-134 induction.

The expression levels of miR-134 did not vary in miR-212/132 $2^{-/-}$or WT hippocampal neurons (Fig. 24a). To find out if there is any alteration in the fold induction of miR-134 after neuronal activity induction as observed for IEGs (Fig. 19-22), the levels of miR-134 before and after kainic acid treatment in WT and miR-212/132/- neurons were compared. However no significant differences in fold changes were observed (Fig. 24b). These results indicate that miR134 regulation is not dependent on miR-212/132 function.

a

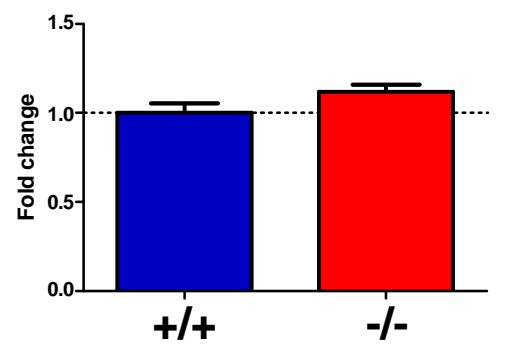

b

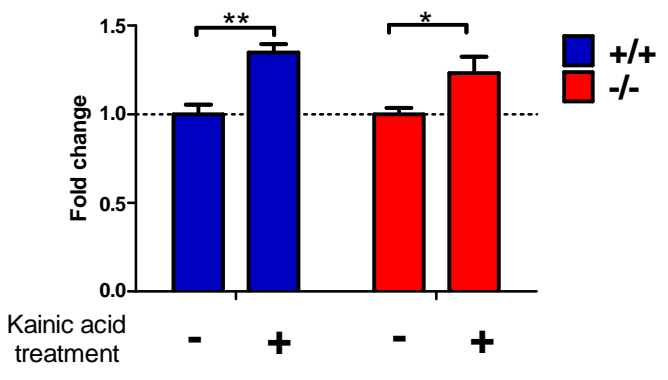

Figure 24: Mature miR-134 levels in cultured hippocampal neurons. (a) Relative miR-134 levels in naïve miR$212 / 132^{-/-}$hippocampal neurons were calculated according to the miR-134 levels in +/+ neurons. (b) $30 \mathrm{M}$ kainic acid treatment was performed for 6 hrs. Relative fold changes were calculated by comparing the time point to the untreated group of the same genotype. Unpaired t-test, two-tailed, *: $\mathrm{p}<0.05, * *: p<0.01, \mathrm{n}=6$.

To further examine the SIRT1-miR-134-CREB pathway, the CREB and pCREB levels in total protein lysates obtained from WT and miR-212/132-/- hippocampal neurons were compared. Kainic acid, as robust neuronal activity inducer, mediates phosphorylation of CREB at Ser133 residue $^{310}$. However, the phosphorylation of CREB upon induction of hippocampal neuron cultures by kainic acid was not prominent enough according to the results I obtained. Therefore, I decided to use forskolin, which is a potent drug in mediation of CREB phosphorylation by increasing the intracellular cAMP concentration ${ }^{326,327}$. To assess the levels of pCREB, an 
antibody that specifically binds to phosphorylated Ser-133 residue was used. The pCREB levels were normalized by total CREB levels. The phosphorylation of CREB was mediated successfully in WT neurons as well as in miR-212/132-/- neurons (Fig. 25a, b). The comparison of the absolute levels of pCREB before and after forskolin treatment in WT and miR-212/132 $2^{-1-}$ neurons revealed that the basal levels of pCREB in WT and miR-212/132/- neurons were same. However, interestingly there were lower amount of pCREB in miR-212/132/- neurons upon equal concentration of forskolin treatment compared to WT neurons (Fig. 25d). This data demonstrates that miR-212/132 deficiency results in the attenuation of CREB phosphorylation.

If SIRT1 were a target of miR-212/132 family in hippocampal neurons, then an upregulation of SIRT1 would be expected in miR-212/132/- neurons. The forskolin treatment did not modulate the levels of SIRT1 in WT and miR-212/132 $2^{--}$neurons (Fig. 25c). This is consistent with the previous study showing that the SIRT1 levels in U2OS cells did not change after $1 \mathrm{hrs}$ of forskolin treatment ${ }^{328}$. When SIRT1 levels in untreated WT neurons are compared with the levels in miR-212/132/- neurons no statistical difference was observed, although the level in miR-212/132 $2^{--}$neurons was tend to be lower (Fig. 25e). Interestingly the difference in the SIRT1 levels between the forskolin treated WT and miR-212/132/- neurons was more prominent, thus statistically significant (Fig. 25e). Therefore SIRT1 would be slightly downregulated in the absence of miR-212/132 and this effect would be enhanced upon forskolin treatment. Since the observation is opposite than the expectation of higher SIRT1 levels in miR$212 / 132^{-/-}$neurons, it is possible that SIRT1 level is influenced by miR-212/132 in an indirect manner. 

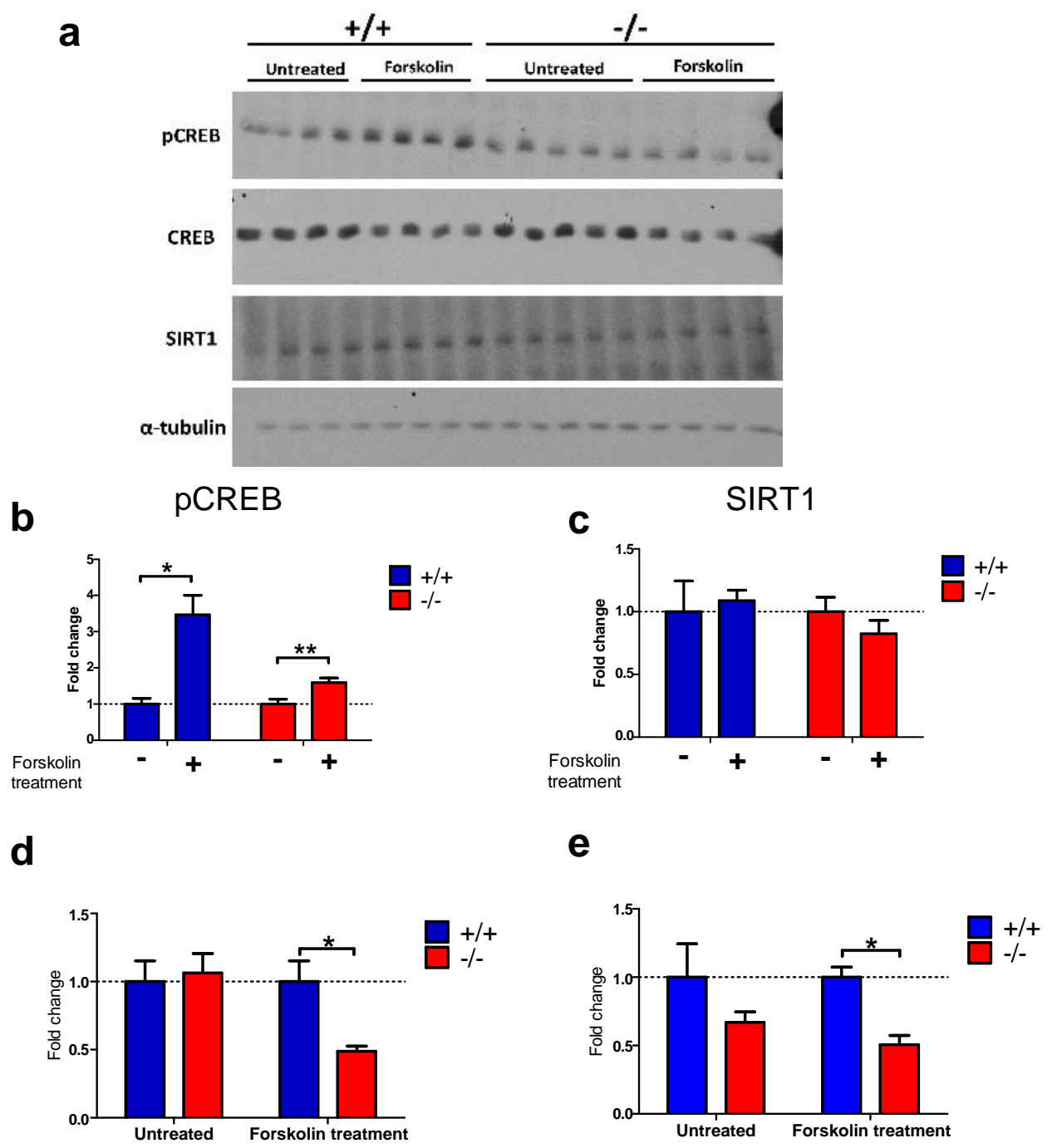

Figure 25: Western blotting for CREB, pCREB and SIRT1. (a) $10 \quad \mathrm{M}$ of forskolin treatment was performed on cultured hippocampal neurons for $30 \mathrm{~min}$. DMSO was applied to untreated group of cells. Equal amount of total protein was loaded into each lane, which represents a replica. Densitometry analysis was performed by Fiji software. (b, d) pCREB level was normalized according to the CREB levels. (c, e) SIRT1 level was normalized according to the $\alpha$-tubulin levels. The pCREB (b) and SIRT1 (c) levels in forskolin untreated groups of both genotypes were set to 1 . Relative amounts of pCREB and SIRT1 was calculated according to the untreated group. The pCREB (d) and SIRT1 (e) levels in untreated and forskolin treated groups were compared for different genotypes by setting the protein amount of $+/+$ group to 1 and calculating the protein amount in -/- accordingly. (b) Paired t-test, two-tailed, *: $p<0.05$, **: $p<0.01$, (d,e) Bonferroni multiple comparison test, *:p<0.05. 


\section{Discussion}

\section{DISCUSSION}

In this thesis the in vivo functions of miR-212/132 family with particular focus on mechanisms of learning and memory were analyzed by using the miR-212/132 knockout mouse line. High expression levels in the brain compared to peripheral organs, and observed expression pattern in the forebrain suggest a possible role of miR-212/132 in cognitive functions. Behavioral assays demonstrated impairment in long-term spatial memory in miR-212/132/- mice, and electrophysiological assays revealed enhanced short-term synaptic facilitation and long-term potentiation (LTP) in Schaffer collateral pathway of miR-212/132/- hippocampal slices. The regulation of immediate early genes, which reside at the forefront of the factors that mediate the neuronal activity dependent changes in neurons, are disrupted upon the lack of miR-212/132 activity. In miR-212/132/- hippocampal neurons c-Fos, Arc and BDNF levels are downregulated. However, 6 hrs of pharmaceutical neuronal activity induction results in abnormally higher levels of c-Fos and Arc transcripts in miR-212/132 $2^{-/-}$neurons. The phosphorylation of the transcription factor cyclic AMP response element binding protein (CREB), which regulates the expression of c-Fos, Arc and BDNF, is attenuated in miR-212/132 $2^{-/-}$hippocampal neurons. Therefore altered CREB signaling pathway is likely to be the underlying molecular defect in miR-212/132-/neurons causing observed behavioral and electrophysiological phenotypes.

\subsection{The correlation between miR-212/132 expression level and phenotype in various tissues}

The expression of miR-212/132 was detected in various organs with strongest expression being in the brain and the pituitary gland. However the abundance of the miRNA expression in a given tissue does not necessarily correlate with the observed loss-of-function phenotypes. Histological analyses of the brain of 6 weeks old mutant mice did not reveal any overt phenotype. In contrast, in the mammary gland that expresses these microRNAs at a very low level, an obvious phenotype was detected. MiR-212/132 expression in the mammary gland is restricted to a small number of stromal cells. The pubertal ductal outgrowth during the mammary gland development is defective in miR-212/132 $2^{-/-}$female mice, which leads to impaired nourishment of their pups. Both miR-212 and miR-132 target matrix metalloproteinase 9 (MMP9) in stromal 
cells. In WT mammary glands, high MMP9 expression in the periductal stroma of terminal end buds (TEB) and low MMP9 expression in the periductal stroma of growth-quiescent ducts was observed. However, in the absence of miR-212/132, high MMP9 expression can be observed in both periductal stromal cells of TEB and fibroblasts scattered in the fat pad. MMP9 activates the latent tumor growth factor- $\beta$ (TGF- $\beta$ ) which has a role in the suppression of epithelial cell division. Alteration in the regulation of MMP9 expression within the mammary glands of miR$212 / 132^{-/-}$mice causes hyperactivation of TGF- $\beta$ signaling pathway, which in turn prevents ductal tree outgrowth ${ }^{115}$.

The expression of a miRNA can be enhanced upon stress. MiR-212/132 was shown to be upregulated in failing human heart ${ }^{329}$. The upregulation of miR-212/132 was observed in cultured primary cardiomyocytes upon treatment with hypertrophy inducing agents such as angiotensin-2, insulin-like growth factor-1, phenylephrine/isoprenaline or fetal calf serum. Transaortic constriction (TAC) operation, which induces cardiac hypertrophy in vivo, increases the levels of miR-212/132 family in the heart as well. Overexpression of miR-212/132 in cardiomyocytes acts like an acute hypertrophy stimulant in vivo, which causes enlargement of the heart and eventually the heart failure. However, miR-212/132 $2^{--}$mice are protected against TAC induced hypertrophy and consequent heart failure. FoxO3 is an anti-hypertrophic and pro-autophagic transcription factor which is targeted by miR-212/132 family. The upregulation of miR-212/132 under hypertrophy inducing conditions or the overexpression of miR-212/132 in cardiomyocytes decreases the levels of FoxO3 expression. Inhibition of autophagy in these conditions contributes to the enlargement of cardiomyocytes in size and the development of hypertrophy ${ }^{330}$.

These phenotypes in miR-212/132/- mice suggest that miR-212/132 activity is required during the regulation of pubertal ductal outgrowth and for the hypertrophic response of cardiomyocytes. As demonstrated in these phenotypes, the restricted spatiotemporal expression of miR-212/132 is essential for the maintenance of proper homeostasis.

\section{2. miR-212/132 and compensation of gene function}

Genomic deletion of most miRNA genes in C.elegans revealed that majority of the miRNAs are dispensable for growth and viability ${ }^{331}$. The same observation is also valid for the majority of the miRNA knockout mouse models ${ }^{332}$. The redundancy among different miRNAs 
which target the same transcripts can be the reason of the observed dispensable roles of particular miRNAs. Such redundancy is observed for miR-17-92 and miR-106b-25 clusters. MiR-106b-25 knockout mice have no vital phenotype, however, deletion of both miR-17-92 and miR-106b-25 clusters result in embryonic lethality at midgestation ${ }^{333}$. Mice that lacks either miR-133a-1 or miR-133a-2 exhibit no phenotype, while ablation of both miRNAs results in heart failure ${ }^{334}$.

MiRNAs with different seed sequences that act on the same target mRNA can regulate the particular transcript in the same manner. Moreover, different miRNAs can influence different cellular networks that consequently have a similar effect on cellular processes. For example miR208b and miR-499, which have 1 nucleotide difference in their seed sequences, were also shown to be redundant in the determination of muscle fiber identity ${ }^{335}$. MiRNAs with different targets may influence the same cellular processes through different molecular networks. For instance, cytosine is found at the $4^{\text {th }}$ position of miR-141 seed sequence, where there is uracil at the same position of miR-200c. It was suggested that although miR-141 and miR-200c have different targets, their impact on cellular division is the same ${ }^{336}$.

MiR-212 and miR-132 constitute a miRNA family. They have the identical seed sequences, but their 3' end has 4 nucleotide differences (Fig. 2). It is known that 3' region of a miRNA has a crucial role in determining the target specificity of the miRNAs ${ }^{337}$. Therefore, although it is likely that both miR-212 and miR-132 have same sets of target mRNAs, it is inappropriate to claim without experimental validation that miR-132 and miR-212 functions can be compensatory to each other. Nevertheless, to avoid any potential redundancy effect, genomic sequences encoding both miR-212 and miR-132 were deleted in the mutant mouse line studied here.

As explained in detail in the sections 1.2.2.1 and 1.2.2.4.1, the previous studies demonstrating the roles of miR-212/132 in neuronal morphology and synaptic transmission yielded several apparently contradictory data so far. The inconsistencies between the results of these studies might be explained based on the differences in the type of neurons used (e.g. cortical versus hippocampal), in the maturation state of those cultured neurons, the applied culturing conditions, and the targeting approaches used in order to obtain the miR-212/132 gainor loss-of-function phenotypes. Moreover, most of these studies focused on the function of the miR-132 alone while the fact that simultaneous expression of miR-212 together with miR-132 
under normal conditions was ignored. This might be especially important in the miR-132-specific knockdown studies since an intact miR-212 in these neurons might lead to functional redundancy. Additionally transfecting neurons with a high concentration of antisense oligonucleotides or sponge vectors to knock-down miR-212 and/or miR-132 may suffer from non-specific bindings of these molecules, thus showing unavoidable off-target effects ${ }^{338,339}$. Transfecting cells with miRNA mimics to mediate an overexpression might also be altering the endogenous miRNA biogenesis pathway ${ }^{340}$. Among all the applied techniques so far, genomic deletion of miR-212/132 is the cleanest system for studying the loss-of-function phenotypes, since this approach avoids various uncontrollable parameters associated with knockdown or other similar methods.

\section{3. miRNAs with role in adaptation}

Extensive research on miRNAs suggests that miRNAs regulate cellular behavior under physiologic and/or pathophysiologic stress ${ }^{332}$. MiR-208a knockout mice show no aberrant defect in heart development or baseline function. However, its functional importance is revealed only when the cardiac hypertrophy is experimentally induced ${ }^{25}$. Deletion of miR-143 and miR-145 in mice does not lead to an alteration in smooth muscle differentiation or vasculogenesis. However, neointima formation does not occur in these mice in response to vascular injury ${ }^{341}$. MiR-212/132 null mutant mice do not show significant phenotype on heart development and the function. However, the heart requires miR-212/132 activity in order to adapt to the conditions that requires mediation of the hypertrophic response.

Environmental stresses encourage adaptation. Therefore, it would be reasonable to consider adaptation as an organismal response to stress. The brain, which is a highly plastic system, is the most complex organ of mammalians. For example in the neocortex of a young man or woman, there are 22.8 and 19.3 billion neurons respectively. The total length of axons in young individuals varies from 150000 to $180000 \mathrm{~km}$. Total number of synapses in human neocortex was calculated as 150 trillion $^{342}$. The complex network of connectivity in a brain must be continuously modified according to the sensory inputs. The plastic feature of a brain, which occurs at the level of axons and dendrites, underlies its ability to adapt the external environment by acquiring, consolidating and recalling the information ${ }^{343}$. The precise control of connectivity 
at each synapse requires dynamic and local regulatory mechanisms. Diverse array of miRNAs is expressed in post-mitotic neurons at the time of synapse development, and they are associated with polyribosomes, where active translation takes place ${ }^{344}$. Additionally, most of the neuronal miRNAs were detected in dendrites as well as in soma ${ }^{345}$. Moreover, an interesting feature of miRNAs is that they do not act as on-off regulators but mostly as fine-tuners ${ }^{346,347}$. Their activity does not exclusively result in the degradation of its target but translational silencing ${ }^{1}$. This feature of miRNAs fits with the scenario that mRNAs must be regulated dynamically by silencing the translation and storing or translating depending on the state of neuron ${ }^{348}$. Therefore, miRNAs are functionally suitable factors that may execute such regulations in synapses. Considering these facts, the lack of any overt structural phenotypes in miR-212/132/- mouse brain is not surprising. Interestingly, the impairment in cognitive, behavior and synaptic transmission suggests that miR$212 / 132$ family is involved in the neuronal network modulation as a component of adaptation of brain to the environmental changes. Moreover the increase in the levels of miR-212/132 expression as the precursor neurons differentiate into the mature neurons and integrate into the circuits supports the role of miR-212/132 in such adaptation mechanism ${ }^{160}$.

\subsection{Roles of miR-212/132 in behavior}

A large battery of behavioral assays was conducted on miR-212/132 null mutant mice and their control littermates. The anxiety (elevated plus maze, Fig. 10a, b), motor balance and control (rotarod test, Fig. 10c), explorative activity (hole-board test, Fig. 10d), sensorimotor gating (prepulse inhibition, Fig. 11a, b), sociability and social memory (social interaction assay, Fig. 11c, d), conditional learning and memory (cued and contextual fear conditioning, Fig. 11e), working memory (8-arm radial maze, Fig. 11f) of these mice were evaluated. None of these assays revealed any significant difference between miR-212/132 $2^{-/-}$and WT mice. This indicates that the genomic deletion of miR-212/132 does not cause substantial sensory deficits and generalized impairments in the behavior.

Open field test revealed that the anxiety level of miR-212/132 $2^{-/-}$mice is not different from WT mice, which is consistent with the elevated plus maze results. However, miR-212/132/- mice cover more distance in the open field than the WT mice. This data indicates that miR-212/132-1mice show more motor activity compared to control littermates. Normally, mice reduce their 
explorative activity as they get familiar with the environment and spend more time grooming or waiting in immobile posture. Mice with frontal cortex or hippocampal lesions show slower habituation to a novel environment, which tend to make animals hyperactive $\mathrm{e}^{349}$. The expression of miR-212/132 was detected in CA1, CA3, and DG regions of the hippocampus as well as in the frontal cortex. Therefore, the deletion of miR-212/132 may alter the flow of information through the hippocampal and cortical circuits, which might eventually display a phenocopy of hippocampal lesions.

Deletion of miR-128 in the forebrain neurons and more specifically, in dopamine-1 receptor (Drd1)-expressing neurons, which has a role in motor control ${ }^{350}$, increases the excitability of neurons. This is reflected as hyperactivity in the open field test, and results in the development of fatal epileptic seizures. Upon Drd1 agonist application, neurons with higher excitability also show increased expression levels of FosB, Dusp1, Arc, Nr4a1, JunB, Jun and Fos12, which are IEGs. Overexpression of miR-128 show the opposite phenotype, such as attenuation of neuronal excitability, decrease in motor activity and seizures ${ }^{351}$. Higher expression levels of IEGs, c-Fos (Fig. 19), Arc (Fig. 20) and BDNF (Fig. 21) in kainic acid treated miR$212 / 132^{-/-}$neurons, indicate a similar increase in the excitability as well. Therefore, the observed hyperactivity in miR-212/132/- mice may be explained by enhanced excitability of neurons, which correlate with the induction of IEGs.

Morris water maze assay demonstrated an increase in the escape latency of miR-212/132 mice although other criteria such as motivation to escape the water, motor activity or visual abilities of mutant mice were same as their WT littermates. These results suggested that, the longterm spatial memory is impaired in the miR-212/132/- mice.

Spatial memory is considered as a subset of declarative memory, which also includes the semantic and episodic memory. Spatial memory is hippocampus and parahippocampus dependent $^{237}$. The neuroimaging studies revealed that neuronal activation in human hippocampus and parahippocampus is greater when trying to find a path rather than following an already known path $^{352}$ and while remembering topographic information than recalling a nonspatial information ${ }^{353}$. Therefore, the medial temporal lobe actively participates in encoding and recalling the spatial memory. The Morris water maze test performed with the miR-212/132-/mice and their control littermates was composed of 8 days of training sessions and the probe trial 
in the consecutive day. The navigational tactics are started to be used by WT mice on the $3^{\text {rd }}$ to $4^{\text {th }}$ day of the training period ${ }^{303}$. After the $4^{\text {th }}$ day, the spatial memory is acquired and encoded. At this time point, the miR-212/132 $2^{-/-}$mice showed worse performance compared to WT mice, indicating that miR-212/132 $2^{-/-}$mice are impaired in acquisition and/or recall of spatial memory.

Long-term spatial memory is generated but not stored in the hippocampus. The amnesic patients with limited hippocampal damage cannot develop new memories, however, they can recall semantic and episodic memories of the past couple of years of pre-amnesia ${ }^{354,355}$. On the other hand, patients with temporal and frontal lobe volume reduction cannot recall autobiographical memory of their early life ${ }^{356}$. Experiments using animal models confirm findings observed on patients with amnesia. If the hippocampal lesion were done 1 day after the eye blink conditioning test, the conditioned response was abolished. However, damaging the hippocampus 4 weeks later had no effect on the response. On the contrary, damage on medial prefrontal cortex did not impair the conditioned response 1 day after but partially after 2 weeks and severely in 4 weeks ${ }^{357}$. These observations assign neocortex as the region where memories are stored for long-term. Expression of miR-212/132 was detected throughout the neocortex of mice (Fig. 7a). This finding hints that miR-212/132 may have a role in the consolidation of the spatial memory in the neocortex for long-term storage. Therefore, to further understand the role of miR-212/132 in memory storage, other behavioral assays testing the long-term memory storage must be conducted in future studies.

\section{5. miR-212/132, neuronal networks and synaptic transmission}

Hippocampus receives inputs from associative cortices via entorhinal cortex (EC). The information that reaches the hippocampus flows through two parallel pathways; trisynaptic pathway (TSP) and monosynaptic pathway (MSP). TSP starts from layer II of EC, then sequentially to DG, CA3, CA1 and back to layer V-VI of EC either directly or through subiculum. MSP starts from layer III of EC, projects to CA1 and to the deep layers of EC again via subiculum or directly ${ }^{236,358}$. CA3 is required for one-trial contextual memory, pattern completion, fine tuning of excitability rate and spatial tuning of CA1 cells. However, MSP can substitute TSP in incremental spatial learning which means the CA3 output is dispensable ${ }^{359}$. CA3 stores associative/sequential information and recalls the information upon exposure to 
partial cue ${ }^{360}$. The CA3 conveys the information to CA1 via Schaffer collaterals. CA1 compares the prior experience dependent information with sensory information arrives from layer III of EC via perforant path, thus mediates the novelty detection ${ }^{361}$. DG and the input from DG to CA3 via perforant path is indispensable for encoding and retrieval of spatial memory ${ }^{362}$.

Short-term synaptic facilitation and enhanced late-phase LTP were observed in Schaffer collateral synapses of miR-212/132 null mutant mice (Fig. 13a, 14b). Short-term facilitation is one type of short-term plasticity. The synaptic efficacy mediated by synaptic facilitation is temporary, which is in the order of a couple of hundred milliseconds. Such fast processes can occur in sensory information processing ${ }^{363}$ and working memory ${ }^{364}$. However, short-term facilitation in miR-212/132 $2^{-/-}$mice was not displayed as an alteration in behavior, as shown in the obtained results of the visual platform assay in Morris water maze (Fig. 12c) and 8-arm radial maze (Fig. 11f) .

BDNF is required for docking of synaptic vesicles at presynaptic compartments ${ }^{365}$ and enhances the glutamate release ${ }^{366}$. It was demonstrated that the mice without functional Myo6 and GIPC6, the factors required for proper BDNF mediated downstream signaling at presynaptic terminals, show lower paired pulse facilitation ${ }^{367}$. Therefore, alteration of BDNF regulation (Fig $21,22 \mathrm{a})$ in miR-212/132/- mice might be associated with the observed increase in synaptic facilitation (Fig. 13a).

In principle, the gain-of-function and the loss-of-function studies for a gene may reveal opposite phenotypes. However, miRNAs may represent an exception to this principle. Since miRNAs are considered as fine tuners, any dysregulation in their expression may have similar phenotypic consequences ${ }^{347}$. Therefore, the detection of higher synaptic facilitation in both miR132 overexpressing neurons in the study of Lambert et al. and in Schaffer collaterals of miR$212 / 132^{-/-}$mice (Fig. 13a) might be the result of miR-132 dysregulation. However one cannot rule out the influence of synaptic connections on synaptic facilitation in the dense network of hippocampal slices, which does not exist in autaptic neuron cultures.

Interestingly Remenyi et al. reported that synapses in Schaffer collateral of miR-212/132 knockout mice show defective synaptic transmission and no synaptic facilitation, which is contradictory to my findings in this study. These differences in the short-term plasticity 
phenotypes might be due to the differences in the gene targeting strategies. Remenyi et al. generated their knockout mouse line by inserting LoxP sites in the $1^{\text {st }}$ intron and $2^{\text {nd }}$ exon of the miR-212/132 expressing non-coding transcript by using an embryonic stem cell line derived from C57B1/6N strain. The deletion of miR-212/132 was mediated by crossing the conditional knockout mice with Cre recombinase expressing transgenic mice under the control of a constitutive promoter ${ }^{162}$. The mouse model that I have used in this study was generated by targeting the miR-212/132 locus in $129 \mathrm{SvJ}$ embryonic stem cells with a targeting vector that disrupts mature miR-212/132 sequences and replaces it with a LacZ reporter gene. After the generation of this mouse line in $129 \mathrm{SvJ}$ background, it was gradually backcrossed into C57B1/6N background. These differences in targeting strategies might be influencing the regulation of the genes located at nearby genomic loci, which can be functionally relevant with the synaptic transmission and the short-term plasticity. Additionally, Remenyi et al. showed that LTP in miR212/132 knockout hippocampal slice is enhanced when the LTP induction is done by theta burst stimulation. This data is supportive to the findings in my thesis. Since the molecular mechanisms of short-term plasticity and LTP are different, the differences in the experimental setups might not be influencing the LTP formation.

Hippocampus is a heterogeneous organ in terms of differential gene expression pattern and connectivity along the dorsoventral axis. Rat and mouse hippocampus can be divided into 3 compartments as ventral $(\mathrm{VH})$, intermediate $(\mathrm{IH})$ and dorsal $(\mathrm{DH})$ hippocampus ${ }^{368}$. The lesion that is restricted to $25 \%$ of the DH is sufficient to impair the spatial memory ${ }^{369}$, while a damage at $\mathrm{VH}$ has no effect on the spatial memory ${ }^{370}$. The place field density in DH is greater compared to $\mathrm{VH}^{370}$. Interestingly, it was shown that mice with lesion in $\mathrm{VH}$ but not in $\mathrm{DH}$ or amygdala enter to the open arm of elevated plus maze more often than control animals, demonstrating altered anxiety ${ }^{371}$. Based on these and other findings, the DH and VH are considered to have distinct roles. DH is involved in navigation and VH is important in emotion and stress ${ }^{372}$. In this respect, observing differential miR-212/132 expression along dorsoventral axis of dentate gyrus (Fig. 8) implies an association with its functional relevance. Conditional miR-212/132 loss-offunction and/or gain-of-function studies restricted to specific sites of the hippocampus must be conducted in order to gain deeper understanding of miR-212/132 functions in neuronal networks regulating these distinct behaviors. 


\section{Discussion}

Interestingly, DH receives a substantial amount of cortical projection from retrosplenial $\operatorname{area}^{373}$, which is involved in the regulation of environmental exploratory behavior ${ }^{374}$. The wholemount LacZ staining on brain demonstrated relatively lower $\beta$-galactosidase expression at retrosplenial area compared to other cortical areas (Fig. 6a, 7a). The expression of miR-212/132 loci at retrosplenial area might be dynamically regulated depending on the exploratory behavior of mice. The expression may increase as mouse explores a novel environment as shown previously for CA1 and DG regions ${ }^{133}$. Since the mice used for $\beta$-galactosidase staining were kept in their home cages, the expression in miR-212/132 loci might not be prominent at retrosplenial area. To address this issue in future studies, mice can be exposed to a novel place or object for a certain time before the dissection of the brain to analyze.

\subsection{Influence of miR-212/132 on c-Fos and Arc expression}

C-Fos is a bZIP transcription factor, which can be used to map the activated neurons in the brain of animals ${ }^{375}$. Conventional knockout of c-Fos shows developmental defects ${ }^{376}$. Therefore, the gene deletion was restricted conditionally to the central nervous system (cFos ${ }^{\mathrm{CNS}}$ ). When a single tetanization at $100 \mathrm{~Hz}$ was performed on acute hippocampal slices, cFos ${ }^{\text {CNS }}$ samples yielded lower LTP compared to controls. However, when the tetanization was repeated 4 times (theta-burst stimulation), no impairment was observed ${ }^{377}$. Therefore, the intensity of the stimulation is crucial for the involvement of c-Fos in LTP formation. Remenyi et al. showed that LTP formation in miR-212/132 knockout Schaffer collaterals is not different from the control group when the induction protocol is single tetanization. However, theta-burst stimulation reveals enhanced LTP in miR-212/132 knockout group ${ }^{162}$. The alteration in the regulation of c-Fos expression in miR-212/132/- neurons (Fig. 22) may explain the altered LTP formation in miR-212/132 loss-of-function mutant hippocampi. Moreover, the c-Fos ${ }^{\mathrm{CNS}}$ mice spent less time in target quadrant of the water maze assay compared to control mice and the freezing response of c-Fos ${ }^{\mathrm{CNS}}$ mice were less on a contextual fear conditioning test $24 \mathrm{hrs}$ after the training. The hippocampus dependent spatial and fear memories are impaired in c-Fos ${ }^{\mathrm{CNS}}$ animals $^{377}$. MiR-212/132/- mice did not show any defect in conditional fear memory (Fig. 11e). Therefore, c-Fos mediated fear memory formation might be miR-212/132 independent. On the other hand, the impaired spatial memory in miR-212/132-- mice may be dependent on the indirect regulation of c-Fos by miR-212/132 family. 


\section{Discussion}

Arc is required to stabilize learning and synaptic plasticity ${ }^{378}$. Arc transcripts are enriched in the postsynaptic compartment ${ }^{379}$. Exposure to a novel environment induces expression of Arc in CA1 pyramidal neurons ${ }^{380}$. It mediates the endocytosis of AMPA receptors (AMPAR) and thus reduces the AMPAR surface expression ${ }^{381}$. The regulation of AMPAR trafficking at postsynaptic membrane by Arc leads synaptic scaling of AMPAR. When there are high levels of neuronal activity in neurons, Arc is induced, which then internalizes the AMPAR in the plasma membrane of synapses. If the neuronal activity is low, Arc levels are downregulated, which leads AMPAR to localize on membranes. Therefore Arc functions as a "homeostasis keeper" that maintains the neuronal response in an optimal range ${ }^{382}$. Arc knockout $\left(\mathrm{Arc}^{-/}\right.$) mice show impairment of long-term memory as it was shown in water maze, contextual and cued fear conditioning, conditioned taste aversion and object recognition assays. However short-term memory remains intact. Moreover, LTP cannot be maintained in $\mathrm{Arc}^{-/-}$mice, and LTD is lower ${ }^{378}$. Remenyi et al. claimed that miR-212/132 has a role in the regulation of postsynaptic AMPAR localization and that the absence of miR-212/132 causes a decrease in AMPAR expression in postsynaptic compartment under basal conditions ${ }^{162}$. Therefore, the dysregulation of Arc in miR212/132 $2^{-/}$hippocampal neurons (Fig. 20, 22) might be the underlying molecular cause of the impaired AMPAR regulation.

\subsection{Functions of miR-212/132 at the molecular level}

CREB is a transcription factor with important roles in neuronal development, synaptic plasticity, learning, memory, addiction, circadian rhythm and neuronal survival ${ }^{383}$. The primary function of CREB in neurons is linking the gene expression to neuronal activity ${ }^{313}$. Neuronal activity dependent $\mathrm{Ca}^{2+}$ influx through voltage gated calcium channels (VGCCs), NMDAR ${ }^{384}$, $\mathrm{AMPAR}^{385}$ and nicotinic acetylcholine receptor ${ }^{386}(\mathrm{nAChR})$ mediates further $\mathrm{Ca}^{2+}$ release from internal calcium stores and activation of downstream signaling factors. Calmodulin that binds to $\mathrm{Ca}^{2+}$ activates the calcium/calmodulin-dependent protein kinase IV (CaMKIV), which in turn phosphorylates $\mathrm{CREB}^{387}$. The CREB-mediated gene activation cannot occur unless Ras-MAPK signaling pathway is activated ${ }^{323}$. Moreover, the rise in the internal $\mathrm{Ca}^{2+}$ concentration activates the adenylyl cyclase, which is a G-protein coupled receptor. Adenylyl cyclase catalyzes cAMP production. Protein kinase A (PKA), which is activated by cAMP phosphorylates CREB ${ }^{388}$. 


\section{Discussion}

CREB function is regulated at the post-translational level through phosphorylation at different residues $^{383}$, as well as post-transcriptionally by miR-134. The expression of miR-134 was shown to be repressed by SIRT1 (Fig. 23). Mice expressing in brain the mutated SIRT1 that have no catalytic activity, had lower levels of BDNF in their hippocampus, and exhibited impaired LTP and spatial memory ${ }^{325}$. Downregulation of BDNF levels upon blockage of miR212 activity was shown in the dorsal striatum ${ }^{219}$. I have also found a decrease in the BNDF transcript levels of untreated miR-212/132 $2^{-/}$hippocampal neurons compared to WT neurons (Fig. 22a). Besides, miR-212/132/- mice have approximately 3 months longer lifespan compared to their WT littermates as shown in fig. 4a. Sir-2, which is the C.elegans homolog of SIRT1, was defined as a factor determining the lifespan ${ }^{389}$. Although this claim was challenged 10 years later $^{390}$, it is clear that SIRT1 is an important factor for healthy aging in mice ${ }^{391}$, which may reflect itself as an increase in lifespan. Since it is known that SIRT1 is targeted by miR-132 ${ }^{146}$, the increase in the lifespan of miR-212/132/- mice might be associated with miR-212/132mediated regulation of SIRT1. In that case the underlying reason of the impaired spatial memory and LTP in miR-212/132/- mice might also be due to the altered regulation of CREB-SIRT1 pathway. However, no significant difference in the levels of miR-134 (Fig. 24) was detectable. Lower level of SIRT1 was detected in forskolin treated miR-212/132/- neurons compared to WT neurons (Fig. 25c, e), which is contrary to the expectations. Therefore, there may be an indirect link between SIRT1 and miR-212/132.

I have shown that forskolin treatment induced CREB phosphorylation is attenuated in miR-212/132 $2^{-/-}$hippocampal neurons and c-Fos, Arc and BDNF transcripts are downregulated in untreated neurons (Fig. 25a, b). Conversely, miR-212 overexpression in human embryonic kidney (HEK) cells enhances forskolin dependent CREB phosphorylation. Moreover, c-Fos is upregulated in HEK cells and the fold induction is enhanced upon forskolin treatment ${ }^{122}$. As explained in section 1.2.2.5.3, potentiation in CREB phosphorylation is TORC dependent, which is a CREB coactivator. TORC acetylation, mediated by $\mathrm{CBP} / \mathrm{p} 300$, protects TORC from proteolytic degradation and the activation of CBP/p300 is cAMP dependent ${ }^{220}$. Increased level of Raf1, which activates adenylyl cyclase, mediates the rise in the intracellular concentration of cAMP in miR-212 overexpressing HEK cells. On the other hand, Raf1 is suppressed by SPRED1, which is targeted by miR-212. Interestingly, the reciprocal interaction between p300 and miR132 was previously shown. Upon infection of monocytes by herpes simplex virus-1 (HSV-1), 
miR-132 suppresses CBP/p300. Eventually, interferon-stimulated genes are attenuated, which in turn enhances the viral replication. Additionally CBP/p300, which interacts with CREB, is required for miR-132 expression ${ }^{144}$. Therefore, the levels of cAMP, CBP/p300, Raf1 and SPRED1 in miR-212/132 $2^{-/}$neurons should be investigated in future studies to reveal if the attenuation of CREB phosphorylation in miR-212/132 $2^{-/}$hippocampal neurons is dependent on a similar cellular mechanism.

The role of BDNF in mature neural circuits was revealed after its discovery as a neurotrophic factor ${ }^{392}$. BDNF enhances the neurotransmitter release ${ }^{367,393-395}$. It increases the postsynaptic response by increasing the NMDA receptor conductance ${ }^{396}$ and AMPAR surface expression $^{397}$ and decreasing the $\mathrm{GABA}_{\mathrm{A}} \mathrm{R}$ surface expression ${ }^{398}$. Besides, BDNF increases the spine density and dendrite outgrowth ${ }^{399-401}$. Basal synaptic transmission and synaptic facilitation are impaired in BDNF knockout $\left(\mathrm{BDNF}^{-/}\right)$mice compared to control mice. LTP measurements on Schaffer collaterals of $\mathrm{BDNF}^{-/-}$hippocampi show lower LTP levels compared to WT hippocampi. The LTP levels can be rescued by application of recombinant BDNF protein ${ }^{402}$. BDNF levels were shown to be upregulated in cortical neurons when miR-132 was knocked down, and this increase was MecP2 dependent. MecP2 is targeted by miR-132 in cultured cortical neurons ${ }^{125}$. However, knocking down miR-212 in dorsal striatum of mice decreased the BDNF levels. MecP2 inhibition in dorsal striatum increases the miR-212/132 expression and miR-212 overexpression decreases MecP2 levels ${ }^{219}$. I have shown that the basal BDNF levels in miR-212/132 $2^{-/}$hippocampal neurons were downregulated. However neither I (Fig. 17), nor Remenyi et al. could confirm the alteration in the levels of MecP2 in miR-212/132 knockout hippocampus and cortex respectively ${ }^{162}$. The contradiction regarding the MecP2 and BDNF levels might be due to the differences in the employed loss-of-function approaches, since the alteration in the level of MecP2 that was observed in a knockdown study cannot be reproduced in these two knockout systems. A technical explanation for this discrepancy might be the possible off-target effects of the employed miR-132 knockdown strategy by Klein et al. Nevertheless the downregulation and change in the fold inductions of BDNF in miR-212/132/- neurons (Fig. 21, 22a) would then be independent of MecP2 but dependent on CREB signaling.

Taken altogether, the expression of miR-212/132 in forebrain is crucial for proper synaptic transmission and spatial memory. The attenuated CREB phosphorylation and alteration 


\section{Discussion}

in the regulation of IEGs in miR-212/132-/- hippocampal neurons suggest that miR-212/132 is primarily important for CREB signaling. 


\section{References}

1 Krol, J., Loedige, I. \& Filipowicz, W. The widespread regulation of microRNA biogenesis, function and decay. Nature reviews. Genetics 11, 597-610, doi:10.1038/nrg2843 (2010).

2 Wightman, B., Ha, I. \& Ruvkun, G. Posttranscriptional regulation of the heterochronic gene lin-14 by lin-4 mediates temporal pattern formation in C. elegans. Cell 75, 855-862 (1993).

3 Lee, R., Feinbaum, R. \& Ambros, V. The C. elegans heterochronic gene lin-4 encodes small RNAs with antisense complementarity to lin-14. Cell 75, 843-854 (1993).

4 Wightman, B., Bürglin, T., Gatto, J., Arasu, P. \& Ruvkun, G. Negative regulatory sequences in the lin-14 3'-untranslated region are necessary to generate a temporal switch during Caenorhabditis elegans development. Genes \& development 5, 1813-1824, doi:10.1101/gad.5.10.1813 (1991). Pasquinelli, A. E. et al. Conservation of the sequence and temporal expression of let-7 heterochronic regulatory RNA. Nature 408, 86-89 (2000).

6 Kozomara, A. \& Griffiths-Jones, S. miRBase: integrating microRNA annotation and deepsequencing data. Nucleic Acids Res. 39, 7, doi:10.1093/nar/gkq1027 (2011).

7 Betel, D., Wilson, M., Gabow, A., Marks, D. \& Sander, C. The microRNA.org resource: targets and expression. Nucleic acids research 36, 53, doi:10.1093/nar/gkm995 (2008).

8 Ruby, J. et al. Large-scale sequencing reveals 21U-RNAs and additional microRNAs and endogenous siRNAs in C. elegans. Cell 127, 1193-1207, doi:10.1016/j.cell.2006.10.040 (2006). sequencing. Cell 129, 1401-1414, doi:10.1016/j.cell.2007.04.040 (2007).

Ruby, J. et al. Evolution, biogenesis, expression, and target predictions of a substantially expanded set of Drosophila microRNAs. Genome research 17, 1850-1864, doi:10.1101/gr.6597907 (2007).

11 Friedman, R., Farh, K., Burge, C. \& Bartel, D. Most mammalian mRNAs are conserved targets of microRNAs. Genome research 19, 92-105, doi:10.1101/gr.082701.108 (2009).

12 Jones-Rhoades, M., Bartel, D. \& Bartel, B. MicroRNAS and their regulatory roles in plants. Annual review of plant biology 57, 19-53, doi:10.1146/annurev.arplant.57.032905.105218 (2006).

Sullivan, C., Grundhoff, A., Tevethia, S., Pipas, J. \& Ganem, D. SV40-encoded microRNAs regulate viral gene expression and reduce susceptibility to cytotoxic T cells. Nature 435, 682-686, doi:10.1038/nature03576 (2005).

14 Mansfield, J. et al. MicroRNA-responsive 'sensor' transgenes uncover Hox-like and other developmentally regulated patterns of vertebrate microRNA expression. Nat Genet 36, 10791083, doi:10.1038/ng1421 (2004).

15 Pfeffer, S. et al. Identification of virus-encoded microRNAs. Science (New York, N.Y.) 304, 734736, doi:10.1126/science.1096781 (2004).

16 Yekta, S., Shih, I. H. \& Bartel, D. MicroRNA-directed cleavage of HOXB8 mRNA. Science (New York, N.Y.) 304, 594-596, doi:10.1126/science.1097434 (2004).

17 Davis, E. et al. RNAi-mediated allelic trans-interaction at the imprinted Rtl1/Peg11 locus. Current biology : $C B$ 15, 743-749, doi:10.1016/j.cub.2005.02.060 (2005).

18 Filipowicz, W., Bhattacharyya, S. \& Sonenberg, N. Mechanisms of post-transcriptional regulation by microRNAs: are the answers in sight? Nature reviews. Genetics 9, 102-114, doi:10.1038/nrg2290 (2008).

19 Grimson, A. et al. MicroRNA targeting specificity in mammals: determinants beyond seed pairing. Molecular cell 27, 91-105, doi:10.1016/j.molcel.2007.06.017 (2007). 
Breving, K. \& Esquela-Kerscher, A. The complexities of microRNA regulation: mirandering around the rules. The international journal of biochemistry \& cell biology 42, 1316-1329, doi:10.1016/j.biocel.2009.09.016 (2010).

21 Tay, Y., Zhang, J., Thomson, A., Lim, B. \& Rigoutsos, I. MicroRNAs to Nanog, Oct4 and Sox2 coding regions modulate embryonic stem cell differentiation. Nature 455, 1124-1128, doi:10.1038/nature07299 (2008).

22 Ameres, S. \& Zamore, P. Diversifying microRNA sequence and function. Nature reviews. Molecular cell biology 14, 475-488, doi:10.1038/nrm3611 (2013).

23 Hwang, H.-W., Wentzel, E. \& Mendell, J. A hexanucleotide element directs microRNA nuclear import. Science (New York, N.Y.) 315, 97-100, doi:10.1038/ncb2010 (2007).

24 Gantier, M. et al. Analysis of microRNA turnover in mammalian cells following Dicer1 ablation. Nucleic Acids Res. 39, 5692-5703, doi:10.1093/nar/gkr148 (2011).

van Rooij, E. et al. Control of stress-dependent cardiac growth and gene expression by a microRNA. Science (New York, N.Y.) 316, 575-579, doi:10.1126/science.1139089 (2007).

Bail, S. et al. Differential regulation of microRNA stability. RNA (New York, N.Y.) 16, 1032-1039, doi:10.1261/rna.1851510 (2010).

Zhang, Z. et al. Uracils at nucleotide position 9-11 are required for the rapid turnover of miR-29 family. Nucleic Acids Res. 39, 4387-4395, doi:10.1093/nar/gkr020 (2011).

Kim, V. MicroRNA biogenesis: coordinated cropping and dicing. Nature reviews. Molecular cell biology 6, 376-385, doi:10.1038/nrm1644 (2005).

Gu, T., Yi, X., Zhao, X., Zhao, Y. \& Yin, J. Alu-directed transcriptional regulation of some novel miRNAs. BMC Genomics 10, 563, doi:10.1186/1471-2164-10-563 (2009).

Dieci, G., Fiorino, G., Castelnuovo, M., Teichmann, M. \& Pagano, A. The expanding RNA polymerase III transcriptome. Trends in genetics : TIG 23, 614-622, doi:10.1016/j.tig.2007.09.001 (2007).

Borchert, G., Lanier, W. \& Davidson, B. RNA polymerase III transcribes human microRNAs. Nat. Struct. Mol. Biol. 13, 1097-1101, doi:10.1038/nsmb1167 (2006).

Lee, Y. et al. MicroRNA genes are transcribed by RNA polymerase II. The EMBO journal 23, 40514060, doi:10.1038/sj.emboj.7600385 (2004).

Cai, X., Hagedorn, C. \& Cullen, B. Human microRNAs are processed from capped, polyadenylated transcripts that can also function as mRNAs. RNA (New York, N.Y.) 10, 1957-1966, doi:10.1261/rna.7135204 (2004).

Ozsolak, F. et al. Chromatin structure analyses identify miRNA promoters. Genes Dev. 22, 31723183, doi:10.1101/gad.1706508 (2008).

Corcoran, D. et al. Features of mammalian microRNA promoters emerge from polymerase I chromatin immunoprecipitation data. PLoS One 4, doi:10.1371/journal.pone.0005279 (2009).

microRNA host genes and transcription units. Genome research 14, 1902-1910, doi:10.1101/gr.2722704 (2004).

Aboobaker, A., Tomancak, P., Patel, N., Rubin, G. \& Lai, E. Drosophila microRNAs exhibit diverse spatial expression patterns during embryonic development. P Natl Acad Sci USA 102, 1801718022, doi:10.1073/pnas.0508823102 (2005).

Baskerville, S. \& Bartel, D. Microarray profiling of microRNAs reveals frequent coexpression with neighboring miRNAs and host genes. RNA (New York, N.Y.) 11, 241-247, doi:10.1261/rna.7240905 (2005). 235-240, doi:10.1038/nature03120 (2004). 
41 Lee, Y. et al. The nuclear RNase III Drosha initiates microRNA processing. Nature 425, 415-419, doi:10.1038/nature01957 (2003).

42 Yang, J.-S. \& Lai, E. Alternative miRNA biogenesis pathways and the interpretation of core miRNA pathway mutants. Mol. Cell 43, 892-903, doi:10.1016/j.molcel.2011.07.024 (2011).

43 Landthaler, M., Yalcin, A. \& Tuschl, T. The human DiGeorge syndrome critical region gene 8 and Its D. melanogaster homolog are required for miRNA biogenesis. Current biology : CB 14, 21622167, doi:10.1016/j.cub.2004.11.001 (2004).

44 Denli, A., Tops, B., Plasterk, R., Ketting, R. \& Hannon, G. Processing of primary microRNAs by the Microprocessor complex. Nature 432, 231-235, doi:10.1038/nature03049 (2004).

45 Han, J. et al. Molecular basis for the recognition of primary microRNAs by the Drosha-DGCR8 complex. Cell 125, 887-901, doi:10.1016/j.cell.2006.03.043 (2006).

46 Lee, Y., Jeon, K., Lee, J.-T., Kim, S. \& Kim, V. MicroRNA maturation: stepwise processing and subcellular localization. The EMBO journal 21, 4663-4670, doi:10.1093/emboj/cdf476 (2002).

47 Lund, E. Nuclear Export of MicroRNA Precursors. Science 303, doi:10.1126/science.1090599 (2004).

$48 \quad$ Yi, R., Qin, Y., Macara, I. \& Cullen, B. Exportin-5 mediates the nuclear export of pre-microRNAs and short hairpin RNAs. Genes Dev. 17, 3011-3016, doi:10.1101/gad.1158803 (2003).

49 Bohnsack, M., Czaplinski, K. \& Gorlich, D. Exportin 5 is a RanGTP-dependent dsRNA-binding protein that mediates nuclear export of pre-miRNAs. RNA (New York, N.Y.) 10, 185-191, doi:10.1261/rna.5167604 (2004).

50 Zeng, Y. \& Cullen, B. Structural requirements for pre-microRNA binding and nuclear export by Exportin 5. Nucleic Acids Res. 32, 4776-4785, doi:10.1093/nar/gkh824 (2004).

51 Bernstein, E., Caudy, A., Hammond, S. \& Hannon, G. Role for a bidentate ribonuclease in the initiation step of RNA interference. Nature 409, 363-366, doi:10.1038/35053110 (2001).

Hutvágner, G. et al. A cellular function for the RNA-interference enzyme Dicer in the maturation of the let-7 small temporal RNA. Science (New York, N.Y.) 293, 834-838, doi:10.1126/science.1062961 (2001).

Grishok, A. et al. Genes and mechanisms related to RNA interference regulate expression of the small temporal RNAs that control C. elegans developmental timing. Cell 106, 23-34, doi:10.1016/S0092-8674(01)00431-7 (2001).

Ketting, R. et al. Dicer functions in RNA interference and in synthesis of small RNA involved in developmental timing in C. elegans. Genes Dev. 15, 2654-2659, doi:10.1101/gad.927801 (2001). Macrae, I. et al. Structural basis for double-stranded RNA processing by Dicer. Science (New York, N.Y.) 311, 195-198, doi:10.1126/science.1121638 (2006).

MacRae, I., Zhou, K. \& Doudna, J. Structural determinants of RNA recognition and cleavage by Dicer. Nat. Struct. Mol. Biol. 14, 934-940, doi:10.1038/nsmb1293 (2007).

Zhang, H., Kolb, F., Jaskiewicz, L., Westhof, E. \& Filipowicz, W. Single processing center models for human Dicer and bacterial RNase III. Cell 118, 57-68, doi:10.1016/j.cell.2004.06.017 (2004). Pase, L. et al. miR-451 regulates zebrafish erythroid maturation in vivo via its target gata2. Blood 113, 1794-1804, doi:10.1182/blood-2008-05-155812 (2009).

Cheloufi, S., Dos Santos, C., Chong, M. \& Hannon, G. A dicer-independent miRNA biogenesis pathway that requires Ago catalysis. Nature 465, 584-589, doi:10.1038/nature09092 (2010).

Yang, J.-S. et al. Conserved vertebrate mir-451 provides a platform for Dicer-independent, Ago2mediated microRNA biogenesis. Proc. Natl. Acad. Sci. U. S. A. 107, 15163-15168, doi:10.1073/pnas.1006432107 (2010).

61 Saito, K., Ishizuka, A., Siomi, H. \& Siomi, M. Processing of pre-microRNAs by the Dicer-1Loquacious complex in Drosophila cells. PLoS Biol. 3, doi:10.1371/journal.pbio.0030235 (2005). 
Förstemann, K. et al. Normal microRNA maturation and germ-line stem cell maintenance requires Loquacious, a double-stranded RNA-binding domain protein. PLoS Biol. 3, doi:10.1371/journal.pbio.0030236 (2005).

Jiang, F. et al. Dicer-1 and R3D1-L catalyze microRNA maturation in Drosophila. Genes Dev. 19, 1674-1679, doi:10.1101/gad.1334005 (2005).

Cenik, E. et al. Phosphate and R2D2 restrict the substrate specificity of Dicer-2, an ATP-driven ribonuclease. Mol. Cell 42, 172-184, doi:10.1016/j.molcel.2011.03.002 (2011).

Fukunaga, R. et al. Dicer partner proteins tune the length of mature miRNAs in flies and mammals. Cell 151, 533-546, doi:10.1016/j.cell.2012.09.027 (2012).

Lee, H. \& Doudna, J. TRBP alters human precursor microRNA processing in vitro. RNA (New York, N.Y.) 18, 2012-2019, doi:10.1261/rna.035501.112 (2012).

Czech, B. \& Hannon, G. Small RNA sorting: matchmaking for Argonautes. Nature reviews. Genetics 12, 19-31, doi:10.1038/nrg2916 (2011).

Johnston, M., Geoffroy, M.-C., Sobala, A., Hay, R. \& Hutvagner, G. HSP90 protein stabilizes unloaded argonaute complexes and microscopic P-bodies in human cells. Mol. Biol. Cell 21, 1462-1469, doi:10.1091/mbc.E09-10-0885 (2010).

$\mathrm{Iki}, \mathrm{T}$. et al. In vitro assembly of plant RNA-induced silencing complexes facilitated by molecular chaperone HSP90. Mol. Cell 39, 282-291, doi:10.1016/j.molcel.2010.05.014 (2010). Iwasaki, S. et al. Hsc70/Hsp90 chaperone machinery mediates ATP-dependent RISC loading of small RNA duplexes. Mol. Cell 39, 292-299, doi:10.1016/j.molcel.2010.05.015 (2010).

Miyoshi, T., Takeuchi, A., Siomi, H. \& Siomi, M. A direct role for Hsp90 in pre-RISC formation in Drosophila. Nat. Struct. Mol. Biol. 17, 1024-1026, doi:10.1038/nsmb.1875 (2010).

Ghildiyal, M., Xu, J., Seitz, H., Weng, Z. \& Zamore, P. Sorting of Drosophila small silencing RNAs partitions microRNA* strands into the RNA interference pathway. RNA (New York, N.Y.) 16, 4356, doi:10.1261/rna.1972910 (2010).

Czech, B. et al. Hierarchical rules for Argonaute loading in Drosophila. Mol. Cell 36, 445-456, doi:10.1016/j.molcel.2009.09.028 (2009).

$\mathrm{Hu}, \mathrm{H}$. et al. Sequence features associated with microRNA strand selection in humans and flies. BMC Genomics 10, 413, doi:10.1186/1471-2164-10-413 (2009).

Frank, F., Sonenberg, N. \& Nagar, B. Structural basis for 5 '-nucleotide base-specific recognition of guide RNA by human AGO2. Nature 465, 818-822, doi:10.1038/nature09039 (2010).

$\mathrm{Mi}$, S. et al. Sorting of small RNAs into Arabidopsis argonaute complexes is directed by the $5^{\prime}$ terminal nucleotide. Cell 133, 116-127, doi:10.1016/j.cell.2008.02.034 (2008).

Lau, N., Lim, L., Weinstein, E. \& Bartel, D. An abundant class of tiny RNAs with probable regulatory roles in Caenorhabditis elegans. Science (New York, N.Y.) 294, 858-862, doi:10.1126/science.1065062 (2001).

Horwich, M. et al. The Drosophila RNA methyltransferase, DmHen1, modifies germline piRNAs and single-stranded siRNAs in RISC. Current biology : CB 17, 1265-1272, doi:10.1016/j.cub.2007.06.030 (2007).

Saito, K. et al. Pimet, the Drosophila homolog of HEN1, mediates 2'-O-methylation of Piwiinteracting RNAs at their 3' ends. Genes Dev. 21, 1603-1608, doi:10.1101/gad.1563607 (2007). Kloosterman, W. \& Plasterk, R. The diverse functions of microRNAs in animal development and disease. Dev. Cell 11, 441-450, doi:10.1016/j.devcel.2006.09.009 (2006).

Tang, G., Reinhart, B., Bartel, D. \& Zamore, P. A biochemical framework for RNA silencing in plants. Genes Dev. 17, 49-63, doi:10.1101/gad.1048103 (2003).

Llave, C., Xie, Z., Kasschau, K. \& Carrington, J. Cleavage of Scarecrow-like mRNA targets directed by a class of Arabidopsis miRNA. Science (New York, N.Y.) 297, 2053-2056, doi:10.1126/science.1076311 (2002). 
83 Dunoyer, P., Lecellier, C.-H., Parizotto, E., Himber, C. \& Voinnet, O. Probing the microRNA and small interfering RNA pathways with virus-encoded suppressors of RNA silencing. The Plant cell 16, 1235-1250, doi:10.1105/tpc.020719 (2004).

84 Souret, F., Kastenmayer, J. \& Green, P. AtXRN4 degrades mRNA in Arabidopsis and its substrates include selected miRNA targets. Mol. Cell 15, 173-183, doi:10.1016/j.molcel.2004.06.006 (2004).

85 German, M. et al. Global identification of microRNA-target RNA pairs by parallel analysis of RNA ends. Nat. Biotechnol. 26, 941-946, doi:10.1038/nbt1417 (2008).

86 Addo-Quaye, C., Eshoo, T., Bartel, D. \& Axtell, M. Endogenous siRNA and miRNA targets identified by sequencing of the Arabidopsis degradome. Current biology : $C B$ 18, 758-762, doi:10.1016/j.cub.2008.04.042 (2008).

87 Jones-Rhoades, M. \& Bartel, D. Computational identification of plant microRNAs and their targets, including a stress-induced miRNA. Mol. Cell 14, 787-799, doi:10.1016/j.molcel.2004.05.027 (2004).

88 Lanet, E. et al. Biochemical evidence for translational repression by Arabidopsis microRNAs. The Plant cell 21, 1762-1768, doi:10.1105/tpc.108.063412 (2009).

89 Brodersen, P. et al. Widespread translational inhibition by plant miRNAs and siRNAs. Science (New York, N.Y.) 320, 1185-1190, doi:10.1126/science.1159151 (2008).

90 Karginov, F. et al. Diverse endonucleolytic cleavage sites in the mammalian transcriptome depend upon microRNAs, Drosha, and additional nucleases. Mol. Cell 38, 781-788, doi:10.1016/j.molcel.2010.06.001 (2010).

91 Shin, C. et al. Expanding the microRNA targeting code: functional sites with centered pairing. Mol. Cell 38, 789-802, doi:10.1016/j.molcel.2010.06.005 (2010).

92 Guo, H., Ingolia, N., Weissman, J. \& Bartel, D. Mammalian microRNAs predominantly act to decrease target mRNA levels. Nature 466, 835-840, doi:10.1038/nature09267 (2010).

93 Place, R., Li, L.-C., Pookot, D., Noonan, E. \& Dahiya, R. MicroRNA-373 induces expression of genes with complementary promoter sequences. Proc. Natl. Acad. Sci. U. S. A. 105, 1608-1613, doi:10.1073/pnas.0707594105 (2008).

94 Henke, J. et al. microRNA-122 stimulates translation of hepatitis C virus RNA. The EMBO journal 27, 3300-3310, doi:10.1038/emboj.2008.244 (2008).

95 Randall, G. et al. Cellular cofactors affecting hepatitis C virus infection and replication. Proc. Natl. Acad. Sci. U. S. A. 104, 12884-12889, doi:10.1073/pnas.0704894104 (2007).

96 Vasudevan, S., Tong, Y. \& Steitz, J. Switching from repression to activation: microRNAs can upregulate translation. Science (New York, N.Y.) 318, 1931-1934, doi:10.1126/science.1149460 (2007).

97 Vasudevan, S. \& Steitz, J. AU-rich-element-mediated upregulation of translation by FXR1 and Argonaute 2. Cell 128, 1105-1118, doi:10.1016/j.cell.2007.01.038 (2007).

$98 \varnothing$ rom, U., Nielsen, F. \& Lund, A. MicroRNA-10a binds the 5'UTR of ribosomal protein mRNAs and enhances their translation. Mol. Cell 30, 460-471, doi:10.1016/j.molcel.2008.05.001 (2008).

99 Bamburg, J. Proteins of the ADF/cofilin family: essential regulators of actin dynamics. Annu. Rev. Cell Dev. Biol. 15, 185-230, doi:10.1146/annurev.cellbio.15.1.185 (1999).

100 Meng, Y. et al. Abnormal spine morphology and enhanced LTP in LIMK-1 knockout mice. Neuron 35, 121-133, doi:10.1016/S0896-6273(02)00758-4 (2002).

101 Schratt, G. et al. A brain-specific microRNA regulates dendritic spine development. Nature 439, 283-289, doi:10.1038/nature04367 (2006).

102 Siegel, G. et al. A functional screen implicates microRNA-138-dependent regulation of the depalmitoylation enzyme APT1 in dendritic spine morphogenesis. Nat. Cell Biol. 11, 705-716, doi:10.1038/ncb1876 (2009). 
103 Ashraf, S., McLoon, A., Sclarsic, S. \& Kunes, S. Synaptic protein synthesis associated with memory is regulated by the RISC pathway in Drosophila. Cell 124, 191-205, doi:10.1016/j.cell.2005.12.017 (2006).

104 Banerjee, S., Neveu, P. \& Kosik, K. A coordinated local translational control point at the synapse involving relief from silencing and MOV10 degradation. Neuron 64, 871-884, doi:10.1016/j.neuron.2009.11.023 (2009).

105 Bagni, C. \& Greenough, W. From mRNP trafficking to spine dysmorphogenesis: the roots of fragile X syndrome. Nature reviews. Neuroscience 6, 376-387, doi:10.1038/nrn1667 (2005).

106 Zalfa, F. et al. The fragile $X$ syndrome protein FMRP associates with BC1 RNA and regulates the translation of specific mRNAs at synapses. Cell 112, 317-327, doi:10.1016/S0092-8674(03)000795 (2003).

107 Edbauer, D. et al. Regulation of synaptic structure and function by FMRP-associated microRNAs miR-125b and miR-132. Neuron 65, 373-384, doi:10.1016/j.neuron.2010.01.005 (2010).

108 Conaco, C., Otto, S., Han, J.-J. \& Mandel, G. Reciprocal actions of REST and a microRNA promote neuronal identity. Proc. Natl. Acad. Sci. U. S. A. 103, 2422-2427, doi:10.1073/pnas.0511041103 (2006).

109 Han, L., Witmer, P., Casey, E., Valle, D. \& Sukumar, S. DNA methylation regulates MicroRNA expression. Cancer Biol. Ther. 6, 1284-1288 (2007).

110 Lim, L., Glasner, M., Yekta, S., Burge, C. \& Bartel, D. Vertebrate microRNA genes. Science (New York, N.Y.) 299, 1540, doi:10.1126/science.1080372 (2003).

111 Lagos-Quintana, M. et al. Identification of tissue-specific microRNAs from mouse. Current biology : CB 12, 735-739, doi:10.1016/S0960-9822(02)00809-6 (2002).

112 Hsu, S.-D. et al. miRNAMap 2.0: genomic maps of microRNAs in metazoan genomes. Nucleic Acids Res. 36, 9, doi:10.1093/nar/gkm1012 (2008).

113 Didiano, D. \& Hobert, O. Perfect seed pairing is not a generally reliable predictor for miRNAtarget interactions. Nat. Struct. Mol. Biol. 13, 849-851, doi:10.1038/nsmb1138 (2006).

114 Didiano, D. \& Hobert, O. Molecular architecture of a miRNA-regulated 3' UTR. RNA (New York, N.Y.) 14, 1297-1317, doi:10.1261/rna.1082708 (2008).

115 Ucar, A. et al. miR-212 and miR-132 are required for epithelial stromal interactions necessary for mouse mammary gland development. Nat. Genet. 42, 1101-1108, doi:10.1038/ng.709 (2010).

116 Vo, N. et al. A cAMP-response element binding protein-induced microRNA regulates neuronal morphogenesis. Proc. Natl. Acad. Sci. U. S. A. 102, 16426-16431, doi:10.1073/pnas. 0508448102 (2005).

117 Remenyi, J. et al. Regulation of the miR-212/132 locus by MSK1 and CREB in response to neurotrophins. The Biochemical journal 428, 281-291, doi:10.1042/BJ20100024 (2010).

118 Magill, S. et al. microRNA-132 regulates dendritic growth and arborization of newborn neurons in the adult hippocampus. Proc. Natl. Acad. Sci. U. S. A. 107, 20382-20387, doi:10.1073/pnas.1015691107 (2010).

119 Impey, S. et al. An activity-induced microRNA controls dendritic spine formation by regulating Rac1-PAK signaling. Mol. Cell. Neurosci. 43, 146-156, doi:10.1016/j.mcn.2009.10.005 (2010).

120 Nudelman, A. et al. Neuronal activity rapidly induces transcription of the CREB-regulated microRNA-132, in vivo. Hippocampus 20, 492-498, doi:10.1002/hipo.20646 (2010).

121 Impey, S. et al. Defining the CREB regulon: a genome-wide analysis of transcription factor regulatory regions. Cell 119, 1041-1054, doi:10.1016/j.cell.2004.10.032 (2004).

122 Hollander, J. et al. Striatal microRNA controls cocaine intake through CREB signalling. Nature 466, 197-202, doi:10.1038/nature09202 (2010).

123 Cheng, H.-Y. M. et al. microRNA modulation of circadian-clock period and entrainment. Neuron 54, 813-829, doi:10.1016/j.neuron.2007.05.017 (2007). 
124 Wayman, G. et al. An activity-regulated microRNA controls dendritic plasticity by downregulating p250GAP. Proc. Natl. Acad. Sci. U. S. A. 105, 9093-9098, doi:10.1073/pnas.0803072105 (2008).

125 Klein, M. et al. Homeostatic regulation of MeCP2 expression by a CREB-induced microRNA. Nat. Neurosci. 10, 1513-1514, doi:10.1038/nn2010 (2007).

126 Wibrand, K. et al. Differential regulation of mature and precursor microRNA expression by NMDA and metabotropic glutamate receptor activation during LTP in the adult dentate gyrus in vivo. The European journal of neuroscience 31, 636-645, doi:10.1111/j.1460-9568.2010.07112.x (2010).

127 Jimenez-Mateos, E. et al. miRNA Expression profile after status epilepticus and hippocampal neuroprotection by targeting miR-132. The American journal of pathology 179, 2519-2532, doi:10.1016/j.ajpath.2011.07.036 (2011).

128 Kawashima, H. et al. Glucocorticoid attenuates brain-derived neurotrophic factor-dependent upregulation of glutamate receptors via the suppression of microRNA-132 expression. Neuroscience 165, 1301-1311 (2010).

129 Numakawa, T. et al. Growth factors stimulate expression of neuronal and glial miR-132. Neurosci. Lett. 505, 242-247, doi:10.1016/j.neulet.2011.10.025 (2011).

130 Mellios, N. et al. miR-132, an experience-dependent microRNA, is essential for visual cortex plasticity. Nat. Neurosci. 14, 1240-1242, doi:10.1038/nn.2909 (2011).

131 Tognini, P., Putignano, E., Coatti, A. \& Pizzorusso, T. Experience-dependent expression of miR132 regulates ocular dominance plasticity. Nat. Neurosci. 14, 1237-1239, doi:10.1038/nn.2920 (2011).

132 Alvarez-Saavedra, M. et al. miRNA-132 orchestrates chromatin remodeling and translational control of the circadian clock. Hum. Mol. Genet. 20, 731-751, doi:10.1093/hmg/ddq519 (2011).

133 Hansen, K. et al. miRNA-132: a dynamic regulator of cognitive capacity. Brain structure \& function 218, 817-831, doi:10.1007/s00429-012-0431-4 (2013).

134 Wang, R.-Y. et al. In vivo knockdown of hippocampal miR-132 expression impairs memory acquisition of trace fear conditioning. Hippocampus 23, 625-633, doi:10.1002/hipo.22123 (2013).

$135 \mathrm{Krol}$, J. et al. Characterizing light-regulated retinal microRNAs reveals rapid turnover as a common property of neuronal microRNAs. Cell 141, 618-631, doi:10.1016/j.cell.2010.03.039 (2010).

136 Wanet, A., Tacheny, A., Arnould, T. \& Renard, P. miR-212/132 expression and functions: within and beyond the neuronal compartment. Nucleic Acids Res. 40, 4742-4753, doi:10.1093/nar/gks151 (2012).

137 Tognini, P. \& Pizzorusso, T. MicroRNA212/132 family: molecular transducer of neuronal function and plasticity. The international journal of biochemistry \& cell biology 44, 6-10, doi:10.1016/j.biocel.2011.10.015 (2012).

138 Molnár, V. et al. MicroRNA-132 targets HB-EGF upon IgE-mediated activation in murine and human mast cells. Cellular and molecular life sciences : CMLS 69, 793-808, doi:10.1007/s00018011-0786-3 (2012).

139 Park, J.-K. et al. miR-132 and miR-212 are increased in pancreatic cancer and target the retinoblastoma tumor suppressor. Biochem. Biophys. Res. Commun. 406, 518-523, doi:10.1016/j.bbrc.2011.02.065 (2011).

140 Carrillo, E. D. et al. Posttranscriptional regulation of the beta2-subunit of cardiac L-type Ca2+ channels by MicroRNAs during long-term exposure to isoproterenol in rats. J. Cardiovasc. Pharmacol. 58, 470-478, doi:10.1097/FJC.0b013e31822a789b (2011).

141 Shaked, l. et al. MicroRNA-132 potentiates cholinergic anti-inflammatory signaling by targeting acetylcholinesterase. Immunity 31, 965-973, doi:10.1016/j.immuni.2009.09.019 (2009). 
142 Anand, S. et al. MicroRNA-132-mediated loss of p120RasGAP activates the endothelium to facilitate pathological angiogenesis. Nat. Med. 16, 909-914, doi:10.1038/nm.2186 (2010).

143 Godoy, J., Nishimura, M. \& Webster, N. J. Gonadotropin-releasing hormone induces miR-132 and miR-212 to regulate cellular morphology and migration in immortalized LbetaT2 pituitary gonadotrope cells. Mol. Endocrinol. 25, 810-820, doi:10.1210/me.2010-0352 (2011).

144 Lagos, D. et al. miR-132 regulates antiviral innate immunity through suppression of the p300 transcriptional co-activator. Nat. Cell Biol. 12, 513-519, doi:10.1038/ncb2054 (2010).

145 Smith, P. et al. MicroRNA-132 loss is associated with tau exon 10 inclusion in progressive supranuclear palsy. Hum. Mol. Genet. 20, 4016-4024, doi:10.1093/hmg/ddr330 (2011).

146 Strum, J. et al. MicroRNA 132 regulates nutritional stress-induced chemokine production through repression of SirT1. Molecular endocrinology (Baltimore, Md.) 23, 1876-1884, doi:10.1210/me.2009-0117 (2009).

147 Huang, Y. et al. MicroRNA regulation of STAT4 protein expression: rapid and sensitive modulation of IL-12 signaling in human natural killer cells. Blood 118, 6793-6802, doi:10.1182/blood-2011-05-356162 (2011).

148 Yang, D. et al. miR-132 regulates the differentiation of dopamine neurons by directly targeting Nurr1 expression. J. Cell Sci. 125, 1673-1682, doi:10.1242/jcs.086421 (2012).

149 Tang, Y. et al. Effect of alcohol on miR-212 expression in intestinal epithelial cells and its potential role in alcoholic liver disease. Alcohol. Clin. Exp. Res. 32, 355-364, doi:10.1111/j.15300277.2007.00584.x (2008).

150 Incoronato, M. et al. miR-212 increases tumor necrosis factor-related apoptosis-inducing ligand sensitivity in non-small cell lung cancer by targeting the antiapoptotic protein PED. Cancer Res. 70, 3638-3646, doi:10.1158/0008-5472.CAN-09-3341 (2010).

151 Nakazawa, T. et al. p250GAP, a novel brain-enriched GTPase-activating protein for Rho family GTPases, is involved in the N-methyl-d-aspartate receptor signaling. Mol. Biol. Cell 14, 29212934, doi:10.1091/mbc.E02-09-0623 (2003).

152 Lamarche, N. \& Hall, A. GAPs for rho-related GTPases. Trends in genetics : TIG 10, 436-440 (1994).

153 Nakayama, A., Harms, M. \& Luo, L. Small GTPases Rac and Rho in the maintenance of dendritic spines and branches in hippocampal pyramidal neurons. The Journal of neuroscience : the official journal of the Society for Neuroscience 20, 5329-5338 (2000).

154 Saneyoshi, T., Fortin, D. \& Soderling, T. Regulation of spine and synapse formation by activitydependent intracellular signaling pathways. Curr. Opin. Neurobiol. 20, 108-115, doi:10.1016/j.conb.2009.09.013 (2010).

155 Ma, X.-M., Wang, Y., Ferraro, F., Mains, R. \& Eipper, B. Kalirin-7 is an essential component of both shaft and spine excitatory synapses in hippocampal interneurons. The Journal of neuroscience : the official journal of the Society for Neuroscience 28, 711-724, doi:10.1523/JNEUROSCI.5283-07.2008 (2008).

156 Ma, X.-M., Huang, J., Wang, Y., Eipper, B. \& Mains, R. Kalirin, a multifunctional Rho guanine nucleotide exchange factor, is necessary for maintenance of hippocampal pyramidal neuron dendrites and dendritic spines. The Journal of neuroscience : the official journal of the Society for Neuroscience 23, 10593-10603 (2003).

157 Xie, Z. et al. Kalirin-7 controls activity-dependent structural and functional plasticity of dendritic spines. Neuron 56, 640-656, doi:10.1016/j.neuron.2007.10.005 (2007).

158 Penzes, P. et al. The neuronal Rho-GEF Kalirin-7 interacts with PDZ domain-containing proteins and regulates dendritic morphogenesis. Neuron 29, 229-242, doi:10.1016/S0896-6273(01)001933 (2001). 
159 Hansen, K., Sakamoto, K., Wayman, G., Impey, S. \& Obrietan, K. Transgenic miR132 alters neuronal spine density and impairs novel object recognition memory. PLoS One 5, doi:10.1371/journal.pone.0015497 (2010).

160 Luikart, B. et al. miR-132 mediates the integration of newborn neurons into the adult dentate gyrus. PLoS One 6, doi:10.1371/journal.pone.0019077 (2011).

161 Pathania, M. et al. miR-132 enhances dendritic morphogenesis, spine density, synaptic integration, and survival of newborn olfactory bulb neurons. PLoS One 7, doi:10.1371/journal.pone.0038174 (2012).

162 Remenyi, J. et al. miR-132/212 knockout mice reveal roles for these miRNAs in regulating cortical synaptic transmission and plasticity. PLoS One 8, doi:10.1371/journal.pone.0062509 (2013).

163 Zhao, C., Deng, W. \& Gage, F. Mechanisms and functional implications of adult neurogenesis. Cell 132, 645-660, doi:10.1016/j.cell.2008.01.033 (2008).

164 Carpentier, P. \& Palmer, T. Immune influence on adult neural stem cell regulation and function. Neuron 64, 79-92, doi:10.1016/j.neuron.2009.08.038 (2009).

165 Monje, M., Toda, H. \& Palmer, T. Inflammatory blockade restores adult hippocampal neurogenesis. Science (New York, N.Y.) 302, 1760-1765, doi:10.1126/science.1088417 (2003).

166 Petreanu, L. \& Alvarez-Buylla, A. Maturation and death of adult-born olfactory bulb granule neurons: role of olfaction. The Journal of neuroscience : the official journal of the Society for Neuroscience 22, 6106-6113 (2002).

167 Björklund, A. \& Dunnett, S. Dopamine neuron systems in the brain: an update. Trends Neurosci. 30, 194-202, doi:10.1016/j.tins.2007.03.006 (2007).

168 Wise, R. Dopamine, learning and motivation. Nature reviews. Neuroscience 5, 483-494, doi:10.1038/nrn1406 (2004).

169 Weidong, L., Shen, C. \& Jankovic, J. Etiopathogenesis of Parkinson disease: a new beginning? The Neuroscientist : a review journal bringing neurobiology, neurology and psychiatry 15, 28-35, doi:10.1177/1073858408319974 (2009).

170 Roy, N. et al. Functional engraftment of human ES cell-derived dopaminergic neurons enriched by coculture with telomerase-immortalized midbrain astrocytes. Nat. Med. 12, 1259-1268, doi:10.1038/nm1495 (2006).

171 Jankovic, J., Chen, S. \& Le, W. D. The role of Nurr1 in the development of dopaminergic neurons and Parkinson's disease. Prog. Neurobiol. 77, 128-138, doi:10.1016/j.pneurobio.2005.09.001 (2005).

172 Lambert, T., Storm, D. \& Sullivan, J. MicroRNA132 modulates short-term synaptic plasticity but not basal release probability in hippocampal neurons. PLoS One 5, doi:10.1371/journal.pone.0015182 (2010).

173 Stevens, C. Neurotransmitter release at central synapses. Neuron 40, 381-388, doi:10.1016/S0896-6273(03)00643-3 (2003).

174 Zola-Morgan, S., Squire, L., Amaral, D. \& Suzuki, W. Lesions of perirhinal and parahippocampal cortex that spare the amygdala and hippocampal formation produce severe memory impairment. The Journal of neuroscience : the official journal of the Society for Neuroscience $\mathbf{9}$, 4355-4370 (1989).

175 Meunier, M., Bachevalier, J., Mishkin, M. \& Murray, E. Effects on visual recognition of combined and separate ablations of the entorhinal and perirhinal cortex in rhesus monkeys. The Journal of neuroscience : the official journal of the Society for Neuroscience 13, 5418-5432 (1993).

176 Mumby, D. G. \& Pinel, J. P. Rhinal cortex lesions and object recognition in rats. Behav. Neurosci. 108, 11-18 (1994).

177 Ennaceur, A., Neave, N. \& Aggleton, J. Neurotoxic lesions of the perirhinal cortex do not mimic the behavioural effects of fornix transection in the rat. Behav. Brain Res. 80, 9-25 (1996). 
178 Ho, V., Lee, J.-A. \& Martin, K. The cell biology of synaptic plasticity. Science (New York, N.Y.) 334, 623-628, doi:10.1126/science.1209236 (2011).

179 Massey, P., Bhabra, G., Cho, K., Brown, M. \& Bashir, Z. Activation of muscarinic receptors induces protein synthesis-dependent long-lasting depression in the perirhinal cortex. The European journal of neuroscience 14, 145-152, doi:10.1046/j.0953-816x.2001.01631.x (2001).

180 Lamprecht, R. \& LeDoux, J. Structural plasticity and memory. Nature reviews. Neuroscience 5, 4554, doi:10.1038/nrn1301 (2004).

181 Caroni, P., Donato, F. \& Muller, D. Structural plasticity upon learning: regulation and functions. Nature reviews. Neuroscience 13, 478-490, doi:10.1038/nrn3258 (2012).

182 Doupe, A. J. \& Kuhl, P. K. Birdsong and human speech: common themes and mechanisms. Annu. Rev. Neurosci. 22, 567-631, doi:10.1146/annurev.neuro.22.1.567 (1999).

183 Johnson, J. \& Newport, E. Critical period effects in second language learning: the influence of maturational state on the acquisition of English as a second language. Cogn. Psychol. 21, 60-99, doi:10.1016/0010-0285(89)90003-0 (1989).

184 Hensch, T. Critical period plasticity in local cortical circuits. Nature reviews. Neuroscience 6, 877888, doi:10.1038/nrn1787 (2005).

185 Hubel, D. \& Wiesel, T. Receptive fields, binocular interaction and functional architecture in the cat's visual cortex. The Journal of physiology 160, 106-154 (1962).

186 Hubel, D. \& Wiesel, T. RECEPTIVE FIELDS OF CELLS IN STRIATE CORTEX OF VERY YOUNG, VISUALLY INEXPERIENCED KITTENS. J. Neurophysiol. 26, 994-1002 (1963).

187 Hubel, D. \& Wiesel, T. The period of susceptibility to the physiological effects of unilateral eye closure in kittens. The Journal of physiology 206, 419-436 (1970).

188 Scott, H. et al. MicroRNA-132 regulates recognition memory and synaptic plasticity in the perirhinal cortex. The European journal of neuroscience 36, 2941-2948, doi:10.1111/j.14609568.2012.08220.x (2012).

189 Barnes, C. A. Memory deficits associated with senescence: a neurophysiological and behavioral study in the rat. J. Comp. Physiol. Psychol. 93, 74-104 (1979).

190 Fanselow, M. \& Tighe, T. Contextual conditioning with massed versus distributed unconditional stimuli in the absence of explicit conditional stimuli. Journal of experimental psychology. Animal behavior processes 14, 187-199 (1988).

191 Chen, C., Kim, J. J., Thompson, R. F. \& Tonegawa, S. Hippocampal lesions impair contextual fear conditioning in two strains of mice. Behav. Neurosci. 110, 1177-1180 (1996).

192 Crawley, J. N. What's wrong with my mouse? : behavioral phenotyping of transgenic and knockout mice. (Wiley-Liss, 2000).

193 Beylin, A. V. et al. The role of the hippocampus in trace conditioning: temporal discontinuity or task difficulty? Neurobiol. Learn. Mem. 76, 447-461, doi:10.1006/nlme.2001.4039 (2001).

194 Bangasser, D., Waxler, D., Santollo, J. \& Shors, T. Trace conditioning and the hippocampus: the importance of contiguity. The Journal of neuroscience : the official journal of the Society for Neuroscience 26, 8702-8706, doi:10.1523/JNEUROSCI.1742-06.2006 (2006).

195 Wallenstein, G. V., Eichenbaum, H. \& Hasselmo, M. E. The hippocampus as an associator of discontiguous events. Trends Neurosci. 21, 317-323 (1998).

196 Reppert, S. \& Weaver, D. Molecular analysis of mammalian circadian rhythms. Annu. Rev. Physiol. (2001).

197 Araki, R. et al. Restricted expression and photic induction of a novel mouse regulatory factor X4 transcript in the suprachiasmatic nucleus. The Journal of biological chemistry 279, 10237-10242, doi:10.1074/jbc.M312761200 (2004). 
198 Zhang, D., Zeldin, D. \& Blackshear, P. Regulatory factor X4 variant 3: a transcription factor involved in brain development and disease. J. Neurosci. Res. 85, 3515-3522, doi:10.1002/jnr.21356 (2007).

199 Nan, X. et al. Transcriptional repression by the methyl-CpG-binding protein MeCP2 involves a histone deacetylase complex. Nature 393, 386-389, doi:10.1038/30764 (1998).

200 Chahrour, M. et al. MeCP2, a key contributor to neurological disease, activates and represses transcription. Science (New York, N.Y.) 320, 1224-1229, doi:10.1126/science.1153252 (2008).

201 Young, J. et al. Regulation of RNA splicing by the methylation-dependent transcriptional repressor methyl-CpG binding protein 2. Proc. Natl. Acad. Sci. U. S. A. 102, 17551-17558, doi:10.1073/pnas.0507856102 (2005).

202 Ogryzko, V., Schiltz, R., Russanova, V., Howard, B. \& Nakatani, Y. The transcriptional coactivators p300 and CBP are histone acetyltransferases. Cell 87, 953-959, doi:10.1016/S00928674(00)82001-2 (1996).

203 Jenuwein, T. \& Allis, C. Translating the histone code. Science (New York, N.Y.) 293, 1074-1080, doi:10.1126/science.1063127 (2001).

204 Agger, K., Christensen, J., Cloos, P. \& Helin, K. The emerging functions of histone demethylases. Curr. Opin. Genet. Dev. 18, 159-168, doi:10.1016/j.gde.2007.12.003 (2008).

205 Mauxion, F., Faux, C. \& Séraphin, B. The BTG2 protein is a general activator of mRNA deadenylation. The EMBO journal 27, 1039-1048, doi:10.1038/emboj.2008.43 (2008).

206 Imataka, H., Gradi, A. \& Sonenberg, N. A newly identified N-terminal amino acid sequence of human elF4G binds poly(A)-binding protein and functions in poly(A)-dependent translation. The EMBO journal 17, 7480-7489, doi:10.1093/emboj/17.24.7480 (1998).

207 Khaleghpour, K. et al. Translational repression by a novel partner of human poly(A) binding protein, Paip2. Mol. Cell 7, 205-216, doi:10.1016/S1097-2765(01)00168-X (2001).

208 Wise, R. Addictive drugs and brain stimulation reward. Annu. Rev. Neurosci. 19, 319-340, doi:10.1146/annurev.ne.19.030196.001535 (1996).

209 Kenny, P. Common cellular and molecular mechanisms in obesity and drug addiction. Nature reviews. Neuroscience 12, 638-651, doi:10.1038/nrn3105 (2011).

210 Rett, A. [On a unusual brain atrophy syndrome in hyperammonemia in childhood]. Wien. Med. Wochenschr. 116, 723-726 (1966).

211 Chahrour, M. \& Zoghbi, H. The story of Rett syndrome: from clinic to neurobiology. Neuron 56, 422-437, doi:10.1016/j.neuron.2007.10.001 (2007).

212 Amir, R. et al. Rett syndrome is caused by mutations in X-linked MECP2, encoding methyl-CpGbinding protein 2. Nat. Genet. 23, 185-188, doi:10.1038/13810 (1999).

213 Chen, W. et al. Derepression of BDNF transcription involves calcium-dependent phosphorylation of MeCP2. Science (New York, N.Y.) 302, 885-889, doi:10.1126/science.1086446 (2003).

214 Martinowich, K. et al. DNA methylation-related chromatin remodeling in activity-dependent BDNF gene regulation. Science (New York, N.Y.) 302, 890-893, doi:10.1126/science.1090842 (2003).

215 Monteggia, L. \& Kavalali, E. Rett syndrome and the impact of MeCP2 associated transcriptional mechanisms on neurotransmission. Biol. Psychiatry 65, 204-210, doi:10.1016/j.biopsych.2008.10.036 (2009).

216 McClung, C. \& Nestler, E. Neuroplasticity mediated by altered gene expression. Neuropsychopharmacology : official publication of the American College of Neuropsychopharmacology 33, 3-17, doi:10.1038/sj.npp.1301544 (2008).

217 Kalivas, P. \& O'Brien, C. Drug addiction as a pathology of staged neuroplasticity. Neuropsychopharmacology : official publication of the American College of Neuropsychopharmacology 33, 166-180, doi:10.1038/sj.npp.1301564 (2008). 
218 Nestler, E. Molecular basis of long-term plasticity underlying addiction. Nature reviews. Neuroscience 2, 119-128, doi:10.1038/35053570 (2001).

219 Im, H.-I., Hollander, J., Bali, P. \& Kenny, P. MeCP2 controls BDNF expression and cocaine intake through homeostatic interactions with microRNA-212. Nat. Neurosci. 13, 1120-1127, doi:10.1038/nn.2615 (2010).

220 Liu, Y. et al. A fasting inducible switch modulates gluconeogenesis via activator/coactivator exchange. Nature 456, 269-273, doi:10.1038/nature07349 (2008).

221 Ding, Q. et al. Raf kinase activation of adenylyl cyclases: isoform-selective regulation. Mol. Pharmacol. 66, 921-928, doi:10.1124/mol.66.4. (2004).

222 King, A. et al. The protein kinase Pak3 positively regulates Raf-1 activity through phosphorylation of serine 338. Nature 396, 180-183, doi:10.1038/24184 (1998).

223 Wakioka, T. et al. Spred is a Sprouty-related suppressor of Ras signalling. Nature 412, 647-651, doi:10.1038/35088082 (2001).

224 Perkins, D. et al. microRNA expression in the prefrontal cortex of individuals with schizophrenia and schizoaffective disorder. Genome Biol. 8, doi:10.1186/gb-2007-8-2-r27 (2007).

$225 \mathrm{Kim}$, A. et al. MicroRNA expression profiling in the prefrontal cortex of individuals affected with schizophrenia and bipolar disorders. Schizophr. Res. 124, 183-191, doi:10.1016/j.schres.2010.07.002 (2010).

226 Botto, L., Moore, C., Khoury, M. \& Erickson, J. Neural-tube defects. The New England journal of medicine 341, 1509-1519, doi:10.1056/NEJM199911113412006 (1999).

227 Zhang, Z. et al. MicroRNAs: potential regulators involved in human anencephaly. The international journal of biochemistry \& cell biology 42, 367-374, doi:10.1016/j.biocel.2009.11.023 (2010).

228 Lee, S.-T. et al. Altered microRNA regulation in Huntington's disease models. Exp. Neurol. 227, 172-179, doi:10.1016/j.expneurol.2010.10.012 (2011).

229 Johnson, R. \& Buckley, N. Gene dysregulation in Huntington's disease: REST, microRNAs and beyond. Neuromolecular Med. 11, 183-199, doi:10.1007/s12017-009-8063-4 (2009).

230 Gillardon, F. et al. MicroRNA and proteome expression profiling in early-symptomatic $\alpha-$ synuclein(A30P)-transgenic mice. Proteomics. Clinical applications 2, 697-705, doi:10.1002/prca.200780025 (2008).

231 Wang, W.-X., Huang, Q., Hu, Y., Stromberg, A. \& Nelson, P. Patterns of microRNA expression in normal and early Alzheimer's disease human temporal cortex: white matter versus gray matter. Acta Neuropathol. 121, 193-205, doi:10.1007/s00401-010-0756-0 (2011).

232 Cogswell, J. et al. Identification of miRNA changes in Alzheimer's disease brain and CSF yields putative biomarkers and insights into disease pathways. Journal of Alzheimer's disease : JAD 14, 27-41 (2008).

233 Buée, L., Bussière, T., Buée-Scherrer, V., Delacourte, A. \& Hof, P. Tau protein isoforms, phosphorylation and role in neurodegenerative disorders. Brain research. Brain research reviews 33, 95-130, doi:10.1016/\$0165-0173(00)00019-9 (2000).

234 Liu, F. \& Gong, C.-X. Tau exon 10 alternative splicing and tauopathies. Mol. Neurodegener. 3, 8, doi:10.1186/1750-1326-3-8 (2008).

235 Andersen, P. The hippocampus book. (Oxford University Press, 2007).

236 Witter, M. et al. Cortico-hippocampal communication by way of parallel parahippocampalsubicular pathways. Hippocampus 10, 398-410, doi:10.1002/1098-1063(2000)10:4<398::AIDHIPO6>3.0.CO;2-K (2000).

237 Squire, L., Stark, C. \& Clark, R. The medial temporal lobe. Annu. Rev. Neurosci. 27, 279-306, doi:10.1146/annurev.neuro.27.070203.144130 (2004). 
238 Steward, O. \& Scoville, S. A. Cells of origin of entorhinal cortical afferents to the hippocampus and fascia dentata of the rat. J. Comp. Neurol. 169, 347-370, doi:10.1002/cne.901690306 (1976).

239 Hjorth-Simonsen, A. \& Jeune, B. Origin and termination of the hippocampal perforant path in the rat studied by silver impregnation. J. Comp. Neurol. 144, 215-232, doi:10.1002/cne.901440206 (1972).

240 Nyakas, C., Luiten, P. G., Spencer, D. G. \& Traber, J. Detailed projection patterns of septal and diagonal band efferents to the hippocampus in the rat with emphasis on innervation of CA1 and dentate gyrus. Brain Res. Bull. 18, 533-545 (1987).

241 Magloczky, Z., Acsady, L. \& Freund, T. F. Principal cells are the postsynaptic targets of supramammillary afferents in the hippocampus of the rat. Hippocampus 4, 322-334, doi:10.1002/hipo.450040316 (1994).

242 Loughlin, S. E., Foote, S. L. \& Grzanna, R. Efferent projections of nucleus locus coeruleus: morphologic subpopulations have different efferent targets. Neuroscience 18, 307-319 (1986).

243 Björklund, A., Hökfelt, T. \& Swanson, L. M. Integrated systems of the CNS. (Elsevier ;

Sole distributors for the USA and Canada, Elsevier Science Pub. Co., 1987).

244 Vertes, R. P., Fortin, W. J. \& Crane, A. M. Projections of the median raphe nucleus in the rat. J. Comp. Neurol. 407, 555-582 (1999).

245 Claiborne, B. J., Amaral, D. G. \& Cowan, W. M. A light and electron microscopic analysis of the mossy fibers of the rat dentate gyrus. J. Comp. Neurol. 246, 435-458, doi:10.1002/cne.902460403 (1986).

246 Acsady, L., Kamondi, A., Sik, A., Freund, T. \& Buzsaki, G. GABAergic cells are the major postsynaptic targets of mossy fibers in the rat hippocampus. J. Neurosci. 18, 3386-3403 (1998).

247 Witter, M. P. Organization of the entorhinal-hippocampal system: a review of current anatomical data. Hippocampus 3 Spec No, 33-44 (1993).

248 Naber, P. A., Lopes da Silva, F. H. \& Witter, M. P. Reciprocal connections between the entorhinal cortex and hippocampal fields CA1 and the subiculum are in register with the projections from CA1 to the subiculum. Hippocampus 11, 99-104, doi:10.1002/hipo.1028 (2001).

249 Pitkanen, A., Pikkarainen, M., Nurminen, N. \& Ylinen, A. Reciprocal connections between the amygdala and the hippocampal formation, perirhinal cortex, and postrhinal cortex in rat. $\mathrm{A}$ review. Ann. N. Y. Acad. Sci. 911, 369-391 (2000).

250 Pikkarainen, M., Ronkko, S., Savander, V., Insausti, R. \& Pitkanen, A. Projections from the lateral, basal, and accessory basal nuclei of the amygdala to the hippocampal formation in rat. J. Comp. Neurol. 403, 229-260 (1999).

251 Swanson, L. W. \& Cowan, W. M. Hippocampo-hypothalamic connections: origin in subicular cortex, not ammon's horn. Science 189, 303-304 (1975).

252 Dolleman-Van der Weel, M. J. \& Witter, M. P. Nucleus reuniens thalami innervates gamma aminobutyric acid positive cells in hippocampal field CA1 of the rat. Neurosci. Lett. 278, 145-148 (2000).

253 Ishizuka, N., Weber, J. \& Amaral, D. G. Organization of intrahippocampal projections originating from CA3 pyramidal cells in the rat. J. Comp. Neurol. 295, 580-623, doi:10.1002/cne.902950407 (1990).

254 Li, X. G., Somogyi, P., Ylinen, A. \& Buzsaki, G. The hippocampal CA3 network: an in vivo intracellular labeling study. J. Comp. Neurol. 339, 181-208, doi:10.1002/cne.903390204 (1994).

255 Fricke, R. \& Cowan, W. M. An autoradiographic study of the commissural and ipsilateral hippocampo-dentate projections in the adult rat. J. Comp. Neurol. 181, 253-269, doi:10.1002/cne.901810204 (1978). 
256 Ishizuka, N. Laminar organization of the pyramidal cell layer of the subiculum in the rat. J. Comp. Neurol. 435, 89-110 (2001).

257 Wyss, J. M. \& Van Groen, T. Connections between the retrosplenial cortex and the hippocampal formation in the rat: a review. Hippocampus 2, 1-11, doi:10.1002/hipo.450020102 (1992).

258 van Groen, T. \& Wyss, J. M. The connections of presubiculum and parasubiculum in the rat. Brain Res. 518, 227-243 (1990).

259 Witter, M. P., Griffioen, A. W., Jorritsma-Byham, B. \& Krijnen, J. L. Entorhinal projections to the hippocampal CA1 region in the rat: an underestimated pathway. Neurosci. Lett. 85, 193-198 (1988).

260 Burwell, R. D. \& Amaral, D. G. Perirhinal and postrhinal cortices of the rat: interconnectivity and connections with the entorhinal cortex. J. Comp. Neurol. 391, 293-321 (1998).

261 Burwell, R. D. Borders and cytoarchitecture of the perirhinal and postrhinal cortices in the rat. J. Comp. Neurol. 437, 17-41 (2001).

262 Insausti, R., Herrero, M. T. \& Witter, M. P. Entorhinal cortex of the rat: cytoarchitectonic subdivisions and the origin and distribution of cortical efferents. Hippocampus 7, 146-183, doi:10.1002/(SICI)1098-1063(1997)7:2\&It;146::AID-HIPO4\&gt;3.0.CO;2-L (1997).

263 Van der Werf, Y. D., Witter, M. P. \& Groenewegen, H. J. The intralaminar and midline nuclei of the thalamus. Anatomical and functional evidence for participation in processes of arousal and awareness. Brain Res. Brain Res. Rev. 39, 107-140 (2002).

264 Scoville, W. B. \& Milner, B. Loss of recent memory after bilateral hippocampal lesions. J. Neurol. Neurosurg. Psychiatry 20, 11-21 (1957).

265 Squire, L. Memory and the hippocampus: a synthesis from findings with rats, monkeys, and humans. Psychol. Rev. 99, 195-231, doi:10.1037//0033-295x.99.2.195 (1992).

266 Richard, G. M. M. Spatial localization does not require the presence of local cues. Learn. Motiv. 12, doi:10.1016/0023-9690(81)90020-5 (1981).

267 Morris, R. G., Schenk, F., Tweedie, F. \& Jarrard, L. E. Ibotenate Lesions of Hippocampus and/or Subiculum: Dissociating Components of Allocentric Spatial Learning. Eur. J. Neurosci. 2, 10161028 (1990).

268 Teng, E. \& Squire, L. Memory for places learned long ago is intact after hippocampal damage. Nature 400, 675-677, doi:10.1038/23276 (1999).

269 O'Keefe, J. \& Dostrovsky, J. The hippocampus as a spatial map. Preliminary evidence from unit activity in the freely-moving rat. Brain Res. 34, 171-175 (1971).

270 Henze, D. A. et al. Intracellular features predicted by extracellular recordings in the hippocampus in vivo. J. Neurophysiol. 84, 390-400 (2000).

271 Langston, R. et al. Development of the spatial representation system in the rat. Science (New York, N.Y.) 328, 1576-1580, doi:10.1126/science.1188210 (2010).

272 Wills, T., Cacucci, F., Burgess, N. \& O'Keefe, J. Development of the hippocampal cognitive map in preweanling rats. Science (New York, N.Y.) 328, 1573-1576, doi:10.1126/science.1188224 (2010).

273 Henriksen, E. et al. Spatial representation along the proximodistal axis of CA1. Neuron 68, 127137, doi:10.1016/j.neuron.2010.08.042 (2010).

274 Boccara, C. et al. Grid cells in pre- and parasubiculum. Nat. Neurosci. 13, 987-994, doi:10.1038/nn.2602 (2010).

275 Sargolini, F. et al. Conjunctive representation of position, direction, and velocity in entorhinal cortex. Science (New York, N.Y.) 312, 758-762, doi:10.1126/science.1125572 (2006).

276 Fyhn, M., Molden, S., Witter, M. P., Moser, E. I. \& Moser, M. B. Spatial representation in the entorhinal cortex. Science 305, 1258-1264, doi:10.1126/science.1099901 (2004).

277 Fyhn, M., Molden, S., Witter, M., Moser, E. \& Moser, M.-B. Spatial representation in the entorhinal cortex. Science (New York, N.Y.) 305, 1258-1264, doi:10.1126/science.1099901 (2004). 
278 Hafting, T., Fyhn, M., Molden, S., Moser, M.-B. \& Moser, E. Microstructure of a spatial map in the entorhinal cortex. Nature 436, 801-806, doi:10.1126/science.1188224 (2005).

279 Taube, J. S., Muller, R. U. \& Ranck, J. B., Jr. Head-direction cells recorded from the postsubiculum in freely moving rats. I. Description and quantitative analysis. J. Neurosci. 10, 420-435 (1990).

280 Solstad, T., Boccara, C., Kropff, E., Moser, M.-B. \& Moser, E. Representation of geometric borders in the entorhinal cortex. Science (New York, N.Y.) 322, 1865-1868, doi:10.1126/science.1166466 (2008).

281 Mcllwain, K., Merriweather, M., Yuva-Paylor, L. \& Paylor, R. The use of behavioral test batteries: effects of training history. Physiol. Behav. 73, 705-717 (2001).

282 Radyushkin, K. et al. Complexin2 null mutation requires a 'second hit' for induction of phenotypic changes relevant to schizophrenia. Genes, brain, and behavior 9, 592-602, doi:10.1111/j.1601183X.2010.00590.x (2010).

283 Radyushkin, K. et al. Neuroligin-3-deficient mice: model of a monogenic heritable form of autism with an olfactory deficit. Genes, Brain and Behavior 8, doi:10.1111/j.1601-183X.2009.00487.x (2009).

284 Radyushkin, K. et al. Genetic ablation of the mammillary bodies in the Foxb1 mutant mouse leads to selective deficit of spatial working memory. The European journal of neuroscience 21, 219-229, doi:10.1111/j.1460-9568.2004.03844.x (2005).

285 Huang, C., Liang, Y. \& Hsu, K. A role for extracellular adenosine in time-dependent reversal of long-term potentiation by low-frequency stimulation at hippocampal CA1 synapses. The Journal of neuroscience : the official journal of the Society for Neuroscience 19, 9728-9738 (1999).

286 Chung, H. et al. G protein-activated inwardly rectifying potassium channels mediate depotentiation of long-term potentiation. Proc. Natl. Acad. Sci. U. S. A. 106, 635-640, doi:10.1073/pnas.0811685106 (2009).

287 Jouvenceau, A., Billard, J.-M., Haditsch, U., Mansuy, I. \& Dutar, P. Different phosphatasedependent mechanisms mediate long-term depression and depotentiation of long-term potentiation in mouse hippocampal CA1 area. The European journal of neuroscience 18, 12791285, doi:10.1046/j.1460-9568.2003.02831.x (2003).

288 Chomczynski, P. \& Sacchi, N. Single-step method of RNA isolation by acid guanidinium thiocyanate-phenol-chloroform extraction. Anal. Biochem. 162, 156-159, doi:10.1006/abio.1987.9999 (1987).

289 Fath, T., Ke, Y., Gunning, P., Götz, J. \& Ittner, L. Primary support cultures of hippocampal and substantia nigra neurons. Nat. Protoc. 4, 78-85, doi:10.1038/nprot.2008.199 (2009).

290 Shipp, S. Structure and function of the cerebral cortex. Curr. Biol. 17, R443-449, doi:10.1016/j.cub.2007.03.044 (2007).

291 Palmer, L., Murayama, M. \& Larkum, M. Inhibitory Regulation of Dendritic Activity in vivo. Frontiers in neural circuits 6, 26, doi:10.3389/fncir.2012.00026 (2012).

292 Kubota, Y. et al. Selective coexpression of multiple chemical markers defines discrete populations of neocortical GABAergic neurons. Cerebral cortex (New York, N.Y. : 1991) 21, 1803 1817, doi:10.1093/cercor/bhq252 (2011).

293 Fukuda, S. et al. Two distinct subpopulations of nestin-positive cells in adult mouse dentate gyrus. The Journal of neuroscience : the official journal of the Society for Neuroscience 23, 93579366 (2003).

294 Garcia, A., Doan, N., Imura, T., Bush, T. \& Sofroniew, M. GFAP-expressing progenitors are the principal source of constitutive neurogenesis in adult mouse forebrain. Nat. Neurosci. 7, 12331241, doi:10.1038/nn1340 (2004). 
295 Suh, H. et al. In vivo fate analysis reveals the multipotent and self-renewal capacities of Sox2+ neural stem cells in the adult hippocampus. Cell stem cell 1, 515-528, doi:10.1016/j.stem.2007.09.002 (2007).

296 Atkins, N., Miller, C., Owens, J. \& Turek, F. Non-laser capture microscopy approach for the microdissection of discrete mouse brain regions for total RNA isolation and downstream nextgeneration sequencing and gene expression profiling. Journal of visualized experiments : JoVE, doi:10.3791/3125 (2011).

297 File, S. \& Wardill, A. Validity of head-dipping as a measure of exploration in a modified holeboard. Psychopharmacologia 44, 53-59, doi:10.1007/BF00421184 (1975).

298 Fruhstorfer, H., Soveri, P. \& Jarvilehto, T. Short-term habituation of the auditory evoked response in man. Electroencephalogr. Clin. Neurophysiol. 28, 153-161 (1970).

299 Geyer, M., Swerdlow, N., Mansbach, R. \& Braff, D. Startle response models of sensorimotor gating and habituation deficits in schizophrenia. Brain Res. Bull. 25, 485-498, doi:10.1016/03619230(90)90241-Q (1990).

300 Fanselow, M. S. Conditioned and unconditional components of post-shock freezing. Pavlov. J. Biol. Sci. 15, 177-182 (1980).

301 Henderson, N. D. Prior treatment effects on open field behaviour of mice--a genetic analysis. Anim. Behav. 15, 364-376 (1967).

302 Morris, R. Developments of a water-maze procedure for studying spatial learning in the rat. J. Neurosci. Methods 11, 47-60 (1984).

303 Janus, C. Search strategies used by APP transgenic mice during navigation in the Morris water maze. Learning \& memory (Cold Spring Harbor, N.Y.) 11, 337-346, doi:10.1101/Im.70104 (2004).

304 Wolfer, D., Stagljar-Bozicevic, M., Errington, M. \& Lipp, H.-P. Spatial Memory and Learning in Transgenic Mice: Fact or Artifact? News in physiological sciences : an international journal of physiology produced jointly by the International Union of Physiological Sciences and the American Physiological Society 13, 118-123 (1998).

305 Wu, L. \& Saggau, P. Presynaptic calcium is increased during normal synaptic transmission and paired-pulse facilitation, but not in long-term potentiation in area CA1 of hippocampus. The Journal of neuroscience : the official journal of the Society for Neuroscience 14, 645-654 (1994).

306 Kamiya, H. \& Zucker, R. Residual Ca2+ and short-term synaptic plasticity. Nature 371, 603-606, doi:10.1038/371603a0 (1994).

307 Malenka, R. \& Bear, M. LTP and LTD: an embarrassment of riches. Neuron 44, 5-21, doi:10.1016/j.neuron.2004.09.012 (2004).

308 Vincent, P. \& Mulle, C. Kainate receptors in epilepsy and excitotoxicity. Neuroscience 158, 309323, doi:10.1016/j.neuroscience.2008.02.066 (2009).

309 Ramamoorthi, K. et al. Npas4 regulates a transcriptional program in CA3 required for contextual memory formation. Science 334, 1669-1675, doi:10.1126/science.1208049 (2011).

310 Rajadhyaksha, A. et al. L-Type $\mathrm{Ca}(2+)$ channels are essential for glutamate-mediated CREB phosphorylation and c-fos gene expression in striatal neurons. The Journal of neuroscience : the official journal of the Society for Neuroscience 19, 6348-6359 (1999).

311 Kandel, E. R. The molecular biology of memory storage: a dialogue between genes and synapses. Science 294, 1030-1038, doi:10.1126/science.1067020

294/5544/1030 [pii] (2001).

312 Nguyen, P. V., Abel, T. \& Kandel, E. R. Requirement of a critical period of transcription for induction of a late phase of LTP. Science 265, 1104-1107 (1994). 
313 Flavell, S. \& Greenberg, M. Signaling mechanisms linking neuronal activity to gene expression and plasticity of the nervous system. Annu. Rev. Neurosci. 31, 563-590, doi:10.1146/annurev.neuro.31.060407.125631 (2008).

$314 \mathrm{Li}, \mathrm{Y}$.-J. et al. Alterations of serum levels of BDNF-related miRNAs in patients with depression. PLoS One 8, doi:10.1371/journal.pone.0063648 (2013).

315 Feng, J. \& Nestler, E. MeCP2 and drug addiction. Nat. Neurosci. 13, 1039-1041, doi:10.1038/nn0910-1039 (2010).

316 Finkel, T., Deng, C.-X. \& Mostoslavsky, R. Recent progress in the biology and physiology of sirtuins. Nature 460, 587-591, doi:10.1038/nature08197 (2009).

317 Kaeberlein, M., McVey, M. \& Guarente, L. The SIR2/3/4 complex and SIR2 alone promote longevity in Saccharomyces cerevisiae by two different mechanisms. Genes Dev. 13, 2570-2580, doi:10.1101/gad.13.19.2570 (1999).

318 Nakahata, Y. et al. The NAD+-dependent deacetylase SIRT1 modulates CLOCK-mediated chromatin remodeling and circadian control. Cell 134, 329-340, doi:10.1016/j.cell.2008.07.002 (2008).

319 Kim, D. et al. SIRT1 deacetylase protects against neurodegeneration in models for Alzheimer's disease and amyotrophic lateral sclerosis. The EMBO journal 26, 3169-3179, doi:10.1038/sj.emboj.7601758 (2007).

320 Renthal, W. et al. Genome-wide analysis of chromatin regulation by cocaine reveals a role for sirtuins. Neuron 62, 335-348, doi:10.1016/j.neuron.2009.03.026 (2009).

321 Donmez, G., Wang, D., Cohen, D. \& Guarente, L. SIRT1 suppresses beta-amyloid production by activating the alpha-secretase gene ADAM10. Cell 142, 320-332, doi:10.1016/j.cell.2010.06.020 (2010).

322 Jiang, M. et al. Neuroprotective role of Sirt1 in mammalian models of Huntington's disease through activation of multiple Sirt1 targets. Nat. Med. 18, 153-158, doi:10.1038/nm.2558 (2012).

323 West, A., Griffith, E. \& Greenberg, M. Regulation of transcription factors by neuronal activity. Nature reviews. Neuroscience 3, 921-931, doi:10.1038/nrn987 (2002).

324 Gao, J. et al. A novel pathway regulates memory and plasticity via SIRT1 and miR-134. Nature 466, 1105-1109, doi:10.1038/nature09271 (2010).

325 Gao, J. et al. A novel pathway regulates memory and plasticity via SIRT1 and miR-134. Nature 466, 1105-1109, doi:10.1038/nature09271 (2010).

326 Laurenza, A., Sutkowski, E. M. \& Seamon, K. B. Forskolin: a specific stimulator of adenylyl cyclase or a diterpene with multiple sites of action? Trends Pharmacol. Sci. 10, 442-447, doi:10.1016/S0165-6147(89)80008-2 (1989).

327 Montminy, M. Transcriptional regulation by cyclic AMP. Annu. Rev. Biochem. 66, 807-822, doi:10.1146/annurev.biochem.66.1.807 (1997).

328 Gerhart-Hines, Z. et al. The CAMP/PKA pathway rapidly activates SIRT1 to promote fatty acid oxidation independently of changes in NAD(+). Mol. Cell 44, 851-863, doi:10.1016/j.molcel.2011.12.005 (2011).

329 Thum, T. et al. MicroRNAs in the human heart: a clue to fetal gene reprogramming in heart failure. Circulation 116, 258-267, doi:10.1161/CIRCULATIONAHA.107.687947 (2007).

330 Ucar, A. et al. The miRNA-212/132 family regulates both cardiac hypertrophy and cardiomyocyte autophagy. Nature communications 3, 1078, doi:10.1038/ncomms2090 (2012).

331 Miska, E. et al. Most Caenorhabditis elegans microRNAs are individually not essential for development or viability. PLoS genetics 3, doi:10.1371/journal.pgen.0030215 (2007).

332 Mendell, J. \& Olson, E. MicroRNAs in stress signaling and human disease. Cell 148, 1172-1187, doi:10.1016/j.cell.2012.02.005 (2012). 
333 Andrea, V. et al. Targeted Deletion Reveals Essential and Overlapping Functions of the miR17 92 Family of miRNA Clusters. Cell 132, doi:10.1016/j.cell.2008.02.019 (2008).

334 Liu, N. et al. microRNA-133a regulates cardiomyocyte proliferation and suppresses smooth muscle gene expression in the heart. Genes Dev. 22, 3242-3254, doi:10.1101/gad.1738708 (2008).

335 Eva van, R. et al. A Family of microRNAs Encoded by Myosin Genes Governs Myosin Expression and Muscle Performance. Dev. Cell 17, doi:10.1016/j.devcel.2009.10.013 (2009).

336 Kim, Y.-K. et al. TALEN-based knockout library for human microRNAs. Nat. Struct. Mol. Biol. 20, 1458-1464, doi:10.1038/nsmb.2701 (2013).

337 Brennecke, J., Stark, A., Russell, R. \& Cohen, S. Principles of microRNA-target recognition. PLoS Biol. 3, doi:10.1371/journal.pbio.0030085 (2005).

338 Stenvang, J., Petri, A., Lindow, M., Obad, S. \& Kauppinen, S. Inhibition of microRNA function by antimiR oligonucleotides. Silence 3, 1, doi:10.1186/1758-907X-3-1 (2012).

339 Ebert, M. \& Sharp, P. MicroRNA sponges: progress and possibilities. RNA (New York, N.Y.) 16, 2043-2050, doi:10.1261/rna.2414110 (2010).

340 Khan, A. et al. Transfection of small RNAs globally perturbs gene regulation by endogenous microRNAs. Nat. Biotechnol. 27, 549-555, doi:10.1038/nbt.1543 (2009).

341 Xin, M. et al. MicroRNAs miR-143 and miR-145 modulate cytoskeletal dynamics and responsiveness of smooth muscle cells to injury. Genes Dev. 23, 2166-2178, doi:10.1101/gad.1842409 (2009).

342 Pakkenberg, B. et al. Aging and the human neocortex. Exp. Gerontol. 38, 95-99, doi:10.1016/S0531-5565(02)00151-1 (2003).

343 Kandel, E. The molecular biology of memory storage: a dialogue between genes and synapses. Science (New York, N.Y.) 294, 1030-1038, doi:10.1126/science.1067020 (2001).

$344 \mathrm{Kim}$, J. et al. Identification of many microRNAs that copurify with polyribosomes in mammalian neurons. Proc. Natl. Acad. Sci. U. S. A. 101, 360-365, doi:10.1073/pnas.2333854100 (2004).

345 Kye, M.-J. et al. Somatodendritic microRNAs identified by laser capture and multiplex RT-PCR. RNA (New York, N.Y.) 13, 1224-1234, doi:10.1261/rna.480407 (2007).

346 Bartel, D. \& Chen, C.-Z. Micromanagers of gene expression: the potentially widespread influence of metazoan microRNAs. Nature reviews. Genetics 5, 396-400, doi:10.1038/nrg1328 (2004).

347 Karres, J., Hilgers, V., Carrera, I., Treisman, J. \& Cohen, S. The conserved microRNA miR-8 tunes atrophin levels to prevent neurodegeneration in Drosophila. Cell 131, 136-145, doi:10.1016/j.cell.2007.09.020 (2007).

348 McNeill, E. \& Van Vactor, D. MicroRNAs shape the neuronal landscape. Neuron 75, 363-379, doi:10.1016/j.neuron.2012.07.005 (2012).

349 Kolb, B. Some Tests of Response Habituation in Rats with Discrete Lesions to Orbital or Medial Frontal Cortex. Canadian Journal of Psychology-Revue Canadienne De Psychologie 28, 260-267, doi:Doi 10.1037/H0081993 (1974).

350 Gerfen, C. The neostriatal mosaic: multiple levels of compartmental organization in the basal ganglia. Annu. Rev. Neurosci. 15, 285-320, doi:10.1146/annurev.ne.15.030192.001441 (1992).

351 Tan, C. L. et al. MicroRNA-128 Governs Neuronal Excitability and Motor Behavior in Mice. Science 342, doi:10.1126/science.1244193 (2013).

352 Hartley, T., Maguire, E. A., Spiers, H. J. \& Burgess, N. The well-worn route and the path less traveled: Distinct neural bases of route following and wayfinding in humans. Neuron 37, 877-888, doi:Doi 10.1016/S0896-6273(03)00095-3 (2003).

353 Maguire, E. A., Frackowiak, R. S. \& Frith, C. D. Recalling routes around Iondon: activation of the right hippocampus in taxi drivers. J. Neurosci. 17, 7103-7110 (1997). 
354 Kapur, N. \& Brooks, D. J. Temporally-specific retrograde amnesia in two cases of discrete bilateral hippocampal pathology. Hippocampus 9, 247-254, doi:10.1002/(SICI)10981063(1999)9:3<247::AID-HIPO5>3.0.CO;2-W (1999).

355 Squire, L. R., Clark, R. E. \& Bayley, P. J. Medial Temporal Lobe Function and Memory. Cognitive Neurosciences lii, Third Edition, 691-708 (2004).

356 Bayley, P. J., Gold, J. J., Hopkins, R. O. \& Squire, L. R. The neuroanatomy of remote memory. Neuron 46, 799-810, doi:10.1016/j.neuron.2005.04.034 (2005).

357 Takehara, K., Kawahara, S. \& Kirino, Y. Time-dependent reorganization of the brain components underlying memory retention in trace eyeblink conditioning. J. Neurosci. 23, 9897-9905 (2003).

358 Yeckel, M. \& Berger, T. Feedforward excitation of the hippocampus by afferents from the entorhinal cortex: redefinition of the role of the trisynaptic pathway. Proc. Natl. Acad. Sci. U. S. A. 87, 5832-5836, doi:10.1073/pnas.87.15.5832 (1990).

359 Nakashiba, T., Young, J., McHugh, T., Buhl, D. \& Tonegawa, S. Transgenic inhibition of synaptic transmission reveals role of CA3 output in hippocampal learning. Science (New York, N.Y.) 319, 1260-1264, doi:10.1126/science.1151120 (2008).

360 Nakazawa, K. et al. Requirement for hippocampal CA3 NMDA receptors in associative memory recall. Science (New York, N.Y.) 297, 211-218, doi:10.1126/science.1071795 (2002).

361 Kumaran, D. \& Maguire, E. Which computational mechanisms operate in the hippocampus during novelty detection? Hippocampus 17, 735-748, doi:10.1002/hipo.20326 (2007).

362 Lee, I. \& Kesner, R. Encoding versus retrieval of spatial memory: double dissociation between the dentate gyrus and the perforant path inputs into CA3 in the dorsal hippocampus. Hippocampus 14, 66-76, doi:10.1002/hipo.10167 (2004).

363 Fortune, E. \& Rose, G. Roles for short-term synaptic plasticity in behavior. J. Physiol. Paris 96, 539-545, doi:10.1016/S0928-4257(03)00009-3 (2002).

364 Itskov, V., Hansel, D. \& Tsodyks, M. Short-Term Facilitation may Stabilize Parametric Working Memory Trace. Front. Comput. Neurosci. 5, 40, doi:10.3389/fncom.2011.00040 (2011).

365 Pozzo-Miller, L. et al. Impairments in high-frequency transmission, synaptic vesicle docking, and synaptic protein distribution in the hippocampus of BDNF knockout mice. The Journal of neuroscience : the official journal of the Society for Neuroscience 19, 4972-4983 (1999).

366 Li, Y., Zhang, Y., Lester, H., Schuman, E. \& Davidson, N. Enhancement of neurotransmitter release induced by brain-derived neurotrophic factor in cultured hippocampal neurons. The Journal of neuroscience : the official journal of the Society for Neuroscience 18, 10231-10240 (1998).

367 Yano, H. et al. BDNF-mediated neurotransmission relies upon a myosin VI motor complex. Nat. Neurosci. 9, 1009-1018, doi:10.1038/nn1730 (2006).

368 Fanselow, M. S. \& Dong, H.-W. Are the dorsal and ventral hippocampus functionally distinct structures? Neuron 65, 7-19 (2010).

369 Moser, M., Moser, E., Forrest, E., Andersen, P. \& Morris, R. Spatial learning with a minislab in the dorsal hippocampus. Proc. Natl. Acad. Sci. U. S. A. 92, 9697-9701, doi:10.1073/pnas.92.21.9697 (1995).

370 Jung, M., Wiener, S. \& McNaughton, B. Comparison of spatial firing characteristics of units in dorsal and ventral hippocampus of the rat. The Journal of neuroscience : the official journal of the Society for Neuroscience 14, 7347-7356 (1994).

371 Kjelstrup, K. et al. Reduced fear expression after lesions of the ventral hippocampus. Proc. Natl. Acad. Sci. U. S. A. 99, 10825-10830, doi:10.1073/pnas.152112399 (2002).

372 Fanselow, M. \& Dong, H. Are the dorsal and ventral hippocampus functionally distinct structures? Neuron (2010).

373 Cenquizca, L. \& Swanson, L. Spatial organization of direct hippocampal field CA1 axonal projections to the rest of the cerebral cortex. Brain research reviews (2007). 
374 Harker, K. \& Whishaw, I. Impaired place navigation in place and matching-to-place swimming pool tasks follows both retrosplenial cortex lesions and cingulum bundle lesions in rats. Hippocampus 14, 224-231, doi:10.1002/hipo.10159 (2004).

375 Herdegen, T. \& Leah, J. Inducible and constitutive transcription factors in the mammalian nervous system: control of gene expression by Jun, Fos and Krox, and CREB/ATF proteins. Brain research. Brain research reviews 28, 370-490, doi:10.1016/S0165-0173(98)00018-6 (1998).

376 Paylor, R., Johnson, R., Papaioannou, V., Spiegelman, B. \& Wehner, J. Behavioral assessment of cfos mutant mice. Brain Res. 651, 275-282, doi:10.1016/0006-8993(94)90707-2 (1994).

377 Fleischmann, A. et al. Impaired long-term memory and NR2A-type NMDA receptor-dependent synaptic plasticity in mice lacking c-Fos in the CNS. The Journal of neuroscience : the official journal of the Society for Neuroscience 23, 9116-9122 (2003).

378 Plath, N. et al. Arc/Arg3.1 is essential for the consolidation of synaptic plasticity and memories. Neuron 52, 437-444, doi:10.1016/j.neuron.2006.08.024 (2006).

379 Steward, O., Wallace, C., Lyford, G. \& Worley, P. Synaptic activation causes the mRNA for the IEG Arc to localize selectively near activated postsynaptic sites on dendrites. Neuron 21, 741-751, doi:10.1016/S0896-6273(00)80591-7 (1998).

380 Guzowski, J. F., McNaughton, B. L., Barnes, C. A. \& Worley, P. F. Environment-specific expression of the immediate-early gene Arc in hippocampal neuronal ensembles. Nat. Neurosci. 2, 11201124, doi:10.1038/16046 (1999).

381 Chowdhury, S. et al. Arc/Arg3.1 interacts with the endocytic machinery to regulate AMPA receptor trafficking. Neuron 52, 445-459, doi:10.1016/j.neuron.2006.08.033 (2006).

382 Shepherd, J. D. \& Bear, M. F. New views of Arc, a master regulator of synaptic plasticity. Nat. Neurosci. 14, 279-284, doi:10.1038/nn.2708 (2011).

383 Lonze, B. \& Ginty, D. Function and regulation of CREB family transcription factors in the nervous system. Neuron 35, 605-623 (2002).

384 Bading, H., Ginty, D. \& Greenberg, M. Regulation of gene expression in hippocampal neurons by distinct calcium signaling pathways. Science (New York, N.Y.) 260, 181-186, doi:10.1126/science.8097060 (1993).

385 Zirpel, L., Janowiak, M., Veltri, C. \& Parks, T. AMPA receptor-mediated, calcium-dependent CREB phosphorylation in a subpopulation of auditory neurons surviving activity deprivation. The Journal of neuroscience : the official journal of the Society for Neuroscience 20, 6267-6275 (2000).

386 Chang, K. \& Berg, D. Voltage-gated channels block nicotinic regulation of CREB phosphorylation and gene expression in neurons. Neuron 32, 855-865, doi:10.1016/S0896-6273(01)00516-5 (2001).

387 Deisseroth, K., Heist, E. \& Tsien, R. Translocation of calmodulin to the nucleus supports CREB phosphorylation in hippocampal neurons. Nature 392, 198-202, doi:10.1038/32448 (1998).

388 Poser, S. \& Storm, D. Role of Ca2+-stimulated adenylyl cyclases in LTP and memory formation. International journal of developmental neuroscience : the official journal of the International Society for Developmental Neuroscience 19, 387-394 (2001).

389 Tissenbaum, H. \& Guarente, L. Increased dosage of a sir-2 gene extends lifespan in Caenorhabditis elegans. Nature 410, 227-230, doi:10.1038/35065638 (2001).

390 Burnett, C. et al. Absence of effects of Sir2 overexpression on lifespan in C. elegans and Drosophila. Nature 477, 482-485, doi:10.1038/nature10296 (2011).

391 Herranz, D. et al. Sirt1 improves healthy ageing and protects from metabolic syndromeassociated cancer. Nature communications 1, 3, doi:10.1038/ncomms1001 (2010).

392 Barde, Y. A., Edgar, D. \& Thoenen, H. Purification of a new neurotrophic factor from mammalian brain. EMBO J. 1, 549-553 (1982). 
393 Jovanovic, J., Czernik, A., Fienberg, A., Greengard, P. \& Sihra, T. Synapsins as mediators of BDNFenhanced neurotransmitter release. Nat. Neurosci. 3, 323-329, doi:10.1038/73888 (2000).

394 Thakker-Varia, S., Alder, J., Crozier, R., Plummer, M. \& Black, I. Rab3A is required for brainderived neurotrophic factor-induced synaptic plasticity: transcriptional analysis at the population and single-cell levels. The Journal of neuroscience : the official journal of the Society for Neuroscience 21, 6782-6790 (2001).

395 Shen, W. et al. Activity-induced rapid synaptic maturation mediated by presynaptic cdc42 signaling. Neuron 50, 401-414, doi:10.1016/j.neuron.2006.03.017 (2006).

396 Suen, P. et al. Brain-derived neurotrophic factor rapidly enhances phosphorylation of the postsynaptic N-methyl-D-aspartate receptor subunit 1. Proc. Natl. Acad. Sci. U. S. A. 94, 81918195, doi:10.1073/pnas.94.15.8191 (1997).

397 Caldeira, M. et al. Brain-derived neurotrophic factor regulates the expression and synaptic delivery of alpha-amino-3-hydroxy-5-methyl-4-isoxazole propionic acid receptor subunits in hippocampal neurons. The Journal of biological chemistry 282, 12619-12628, doi:10.1074/jbc.M700607200 (2007).

398 Jovanovic, J., Thomas, P., Kittler, J., Smart, T. \& Moss, S. Brain-derived neurotrophic factor modulates fast synaptic inhibition by regulating GABA(A) receptor phosphorylation, activity, and cell-surface stability. The Journal of neuroscience : the official journal of the Society for Neuroscience 24, 522-530, doi:10.1523/JNEUROSCI.3606-03.2004 (2004).

399 Park, H. \& Poo, M.-m. Neurotrophin regulation of neural circuit development and function. Nature reviews. Neuroscience 14, 7-23, doi:10.1038/nrn3379 (2013).

400 Alonso, M., Medina, J. \& Pozzo-Miller, L. ERK1/2 activation is necessary for BDNF to increase dendritic spine density in hippocampal CA1 pyramidal neurons. Learning \& memory (Cold Spring Harbor, N.Y.) 11, 172-178, doi:10.1101/Im.67804 (2004).

401 Rex, C. et al. Brain-derived neurotrophic factor promotes long-term potentiation-related cytoskeletal changes in adult hippocampus. The Journal of neuroscience : the official journal of the Society for Neuroscience 27, 3017-3029, doi:10.1523/JNEUROSCI.4037-06.2007 (2007).

402 Patterson, S. et al. Recombinant BDNF rescues deficits in basal synaptic transmission and hippocampal LTP in BDNF knockout mice. Neuron 16, 1137-1145, doi:10.1016/S08966273(00)80140-3 (1996). 


\section{List of Abbreviations}

\begin{tabular}{|l|l|}
\hline 3'UTR & 3' untranslated region \\
\hline 3R-tau & three microtubule binding repeats \\
\hline 4R-tau & four microtubule binding repeats \\
\hline 5'UTR & $5^{\prime}$ untranslated region \\
\hline Ach & acethylcholine \\
\hline AchE & acetylcholinesterase \\
\hline ACSF & artificial cerebrospinal fluid \\
\hline AMPAR & $\alpha$-amino-3-hydroxy-5-methyl-4-isoxazolepropionic acid receptor \\
\hline BDNF & brain derived neurotrophic factor \\
\hline bFGF & basic fibroblast growth factor \\
\hline BTG2 & B-cell translocation gene 2 \\
\hline CA & cornu Ammonis \\
\hline CaMKIV & calcium/calmodulin-dependent protein kinase IV \\
\hline cAMP & cyclic adenosine monophosphate \\
\hline CCh & carbachol \\
\hline CDS & coding sequences \\
\hline CR & conditional response \\
\hline CRE & cyclic AMP response element \\
\hline CREB & cyclic AMP response element binding protein \\
\hline CS & conditioned stimulus \\
\hline CSDC2 & cold-shock domain containing protein C2 \\
\hline DG & dentate gyrus \\
\hline DH & dorsal hippocampus \\
\hline DIV & day in vitro \\
\hline DPI & days post infection \\
\hline Drd1 & dopamine-1 receptor \\
\hline EC & entorhinal cortex \\
\hline E-LTP & early-LTP \\
\hline EPSC & excitatory postsynaptic current \\
\hline fEPSC & field excitatory postsynaptic potentials \\
\hline FXR1 & fragile X mental retardation related protein 1 \\
\hline GCL & granule cell layer \\
\hline GTC & Guanidin thiocyanate \\
\hline HB-EGF & Heparin-binding EGF-like growth factor \\
\hline HCMV & human cytomegalovirus \\
\hline HEK & human embryonic kidney cells \\
\hline HFS & high frequency stimulation \\
\hline HPF & hippocampal formation \\
\hline HSV-1 & herpes simplex virus-1 \\
\hline & \\
\hline
\end{tabular}


Abbreviations

\begin{tabular}{|l|l|}
\hline IEG & immediate early genes \\
\hline IH & intermediate hippocampus \\
\hline IL-6 & Interleukin-6 \\
\hline I-O & input-output \\
\hline IRES & internal ribosomal entry site \\
\hline ISH & in situ hybridization \\
\hline JARID1A & Jumonji, AT-rich interactive domain 1A \\
\hline KSHV & Kaposi's sarcoma-associated herpesvirus \\
\hline LA & lateral amygdala \\
\hline LFS & low frequency stimulation \\
\hline LIMK1 & Lim-domain-containing protein kinase 1 \\
\hline L-LTP & late long-term potentiation \\
\hline LNA & Locked-nucleic acid \\
\hline Loqs-PA & Loquacious-PA \\
\hline Loqs-PB & Loquacious PB \\
\hline LTD & long-term depression \\
\hline m7Gppp & 7-methyguanosine cap \\
\hline MeCP2 & methyl CpG binding protein 2 \\
\hline MEFs & mouse embryonic fibroblasts \\
\hline mEPSC & miniature excitatory postsynaptic current \\
\hline miRNA & microRNA \\
\hline MMP9 & matrix metalloproteinase 9 \\
\hline MSP & monosynaptic pathway \\
\hline NA & nucleus accumbens \\
\hline nAChR & nicotinic acetylcholine receptor \\
\hline NBQX & 6-nitro-2,3-dioxo-1,4,-dihydrobenzo[f]quinoxaline-7-sulfonamide () \\
\hline NMDAR & $\begin{array}{l}\text { ( } \text {-)-3-(2-carboxycpiperazin-4-yl)propyl-1-phosphonic acid that blocks N-Methyl- } \\
\text { D-aspartic acid receptor }\end{array}$ \\
\hline p120RasGAP & RAS P21 Protein Activator \\
\hline p250GAP & Rac GTPase activating protein \\
\hline p300 & E1A binding protein p300 \\
\hline PABP & polyadenylate binding protein \\
\hline PAIP2A & polyadenylate-binding protein- interacting protein 2 \\
\hline PBS & phosphate Buffered Saline \\
\hline pCREB & phosphorylated cyclic AMP response element binding protein \\
\hline PD & Parkinson's disease \\
\hline PED/PEA-15 & phosphoprotein enriched in astrocytes 15 \\
\hline PKA & protein kinase A \\
\hline PPF & paired pulse facilitation \\
\hline PPRs & paired-pulse ratios \\
\hline pre-miRNA & precursor microRNA \\
\hline & \\
\hline
\end{tabular}


Abbreviations

\begin{tabular}{|l|l|}
\hline PRh & perirhinal cortex \\
\hline pri-miRNA & primary microRNA \\
\hline PSP & progressive supranuclear palsy \\
\hline PTBP2 & polypyrimidine tract binding protein 2 \\
\hline Rac GEF & Rac guanine exchange factor \\
\hline Rb1 & retinoblastoma tumor suppressor 1 \\
\hline REST & repressor element 1 silencing transcription factor \\
\hline RFX4 & regulatory factor X4 \\
\hline RISC & RNA-induced silencing complex \\
\hline RMS & rostral migratory stream \\
\hline RRP & readily releasable pool \\
\hline RSP & retrosplenial area \\
\hline RXF4 & regulatory factor X, 4 \\
\hline SCN & suprachiasmatic nucleus \\
\hline SEPSC & spontaneous excitatory postsynaptic current \\
\hline SGZ & subgranular zone \\
\hline Sir2 & silent information regulator \\
\hline SirT1 & sirtuin 1 \\
\hline s1 & stratum lucidum \\
\hline sl-m & stratum lacunosum-moleculare \\
\hline so & stratum oriens \\
\hline SPRED1 & sprout-related EVH1 domain containing 1 \\
\hline sr & stratum radiatum \\
\hline STAT4 & signal transducer and activator of transcription 4 \\
\hline SVZ & subventricular zone \\
\hline TAC & transaortic constriction \\
\hline tCREB & total cyclic AMP response element binding protein \\
\hline TEB & terminal end buds \\
\hline TEMED & tetramethylethylenediamine \\
\hline TGF- $\beta$ & tumor growth factor- $\beta$ \\
\hline TH & thalamus \\
\hline TNF- $\alpha$ & tumor necrosis factor- $\alpha$ \\
\hline TSP & trisynaptic pathway \\
\hline TT & taenia tecta \\
\hline US & unconditioned stimulus \\
\hline VGCCs & voltage gated calcium channels \\
\hline VH & ventral hippocampus \\
\hline WT & wild type \\
\hline ZO-1 & Zonula occludens 1 \\
\hline & \\
\hline
\end{tabular}




\section{Acknowledgements}

I would like to express my deepest appreciation to my supervisor, Dr. Kamal Chowdhury, for the opportunity he has given me to join his lab and conduct my research. I would additionally like to thank him for his invaluable guidance, support, understanding and patience during my $\mathrm{PhD}$ years.

I would like to thank Dr. Ahmet Uçar to be there whenever I need. I appreciate his guidance and support during my studies. He was like a brother to me.

My heartfelt thanks are also due to Prof. Hannelore Ehrenreich, Dr. Konstantin Radyushkin and Dr. Jeong Seop Rhee for a very fruitful collaboration and very useful discussions.

I wish to thank my thesis committee members, Prof. Ernst Wimmer and Prof. Michael Kessel, for their precious suggestions, guidance and patience. They were always very kind and helpful.

I thank a lot to Sabine Geisendorf for her technical assistance and her great friendship. The conversations we had about nature, future, career, meaning of life, relationships etc. were all unforgettable.

I appreciate the help of Alexandra Kurth in the animal facility taking care of my mouse colonies. She does just a perfect job.

I also thank to Sharif for his assistance in cell culturing and for making me laugh. He was the source of humors in the department.

I would also like to thank Max Planck Society for their financial support and the infrastructure they have provided to conduct my research during my $\mathrm{PhD}$ studies.

I would like to thank to my laboratory mates, Alex, Zeeshan, Tamara, Mehdi, Farnaz, Nazım and Ali, for their help, precious friendship and support in the lab and my experiments.

Eralp, who made me survive the toughest times in Germany, deserves my biggest appreciations. He will always be my best buddy.

I especially thank to Ela Sophie for everything she did for me with all her goodwill. 
My special thanks are to my grandmothers Makbule and Müşerref for their prayers and heartening me whenever I need.

I am so thankful to my parents Sevgi, Cahit and my brother Kerem, who have encouraged me and have made this work possible with their constant help and guidance during my time as a $\mathrm{PhD}$ student. I would like to express my love and gratitude for their everlasting support. 


\section{Figure Legends}

Figure 1: Illustration of microRNA Biogenesis Pathway (Ameres et al. 2013. Permission to reuse the illustration was given by Nature Publishing group. License \#:3297570668022) 5

Figure 2: miR-212/132 sequence and structures. a) The structure of pre-miR-212 and pre-miR132 is depicted. The region, which will become mature miRNA are shaded in red. b) The mature miR-212, miR-132, miR-212* and miR-132* sequences are shown. The seed sequences are typed in red.

Figure 3: Body weight measurement of WT and miR-212/132-/- mice at different ages. The mice were grouped according to their ages as younger than 1 month, 1-2 months old, 2-3 months old and older than 3 months. 2-way ANOVA for age versus genotype interaction $\mathrm{F}_{(3,59)}=0.34, \mathrm{p}=$ 0.8 ; for difference between mice at different age groups $\mathrm{F}_{(3,59)}=26.4, \mathrm{p}<0.0001$; for difference between genotypes $\mathrm{F}_{(1,59)}=0.18, \mathrm{p}=0.67: 1 \mathrm{mo}>\mathrm{x}:+/+, \mathrm{n}=4,-/-, \mathrm{n}=8 ; 2 \operatorname{mos}>\mathrm{x}>1$ mos: $:+/+, \mathrm{n}=16$, $-/-, \mathrm{n}=11 ; 3 \operatorname{mos}>\mathrm{x}>2 \operatorname{mos}:+/+, \mathrm{n}=10,-/-, \mathrm{n}=5 ; \mathrm{x}>3 \operatorname{mos}:+/+, \mathrm{n}=8,-/-, \mathrm{n}=5$.

Figure 4: Survival analysis on miR-212/132-/- mice. The health status of WT and miR-212/132-/mice with ad libitum access to water and food were observed continuously. (a) The date of death of each mouse was recorded and (b) the presence of tumors was evaluated as the cause of death. If an obvious tumor formation was observed, the mice were classified in "tumor formation" group. If the mice were found death in cage or tumor formation was not observed they were grouped as "unknown cause of death". (a) Mantel-Cox test, p=0.0038; Gehan-Breslow-Wilcoxon test, $\mathrm{p}=0.02$. (b) Fisher's exact test, two-sided, $\mathrm{p}=0.76$ 61

Figure 5: The levels of mature miR-212 and miR-132 in different mouse organs. The organs were dissected and RNA isolation was performed. Equal concentration of cDNA was used to perform RT Q-PCR. Sno202 was used as endogenous control. The miR-212 and miR-132 levels in thyroid gland were set to 1 and the fold expressions were calculated accordingly........ 
Figure 6: The staining patterns on miR-212/132 $2^{+/-}$and miR-212/132 $2^{-/-}$brains were compared. (a) Whole mount staining of brains of $+/$ - and -/- mice were visualized from dorsal and ventral sides. RSP: retrosplenial area, TT: taenia tecta, HPF: Hippocampal formation. $(b, c)$ The brain sections, where $\beta$-galactosidase staining was most prominent, were shown here as representative planes. (d, e) Higher magnification pictures on dorsal hippocampus of the sections shown in (b) and (c). The arrows point dotted staining. Scale bars represent $5 \mathrm{~mm}$ in (a), $2 \mathrm{~mm}$ in (b, c) and $500 \mathrm{~m}$ in $(d, e)$.

Figure 7: $\beta$-galactosidase staining on whole adult brain and coronal sections. (a) Whole mount $\beta$ galactosidase staining of 5 weeks-old mouse brain showed miR-212/132 expression in cortical plate. (b-i) $\beta$-galactosidase staining on coronal sections demonstrated LacZ expressing cells in spatially restricted regions of the forebrain (b), which is shown in higher magnification for somatosensory cortex (c), hippocampus (d), CA1 and CA2 regions of hippocampus, CA3 region of hippocampus (f), dentate gyrus (g), striatum (h) and amygdala (i). (c),(d), (h) and (i) are higher magnification pictures of the regions shown within white rectangles in (b). The regions shown within white rectangles in (d) are shown in high magnification in (e), (f), and (g). TT, taenia tecta; SSp, primary somatosensory area; PTLp, posterior parietal association areas; CA, cornu ammonis; DG, dentate gyrus; ST, striatum; LA, lateral amygdalar nucleus. The histological boundaries for cortical layers and hippocampal structures are determined according to the DAPI staining results of the same sections. Scale bars represent $5 \mathrm{~mm}$ in (a), $2 \mathrm{~mm}$ in (b), $500 \mu \mathrm{m}$ in (c) and (d), $200 \mu \mathrm{m}$ in (e-i).

Figure 8: LacZ staining on the coronal sections of different planes of 5-weeks old male (a-e) and female (f-j) miR-212/132-- mouse brains demonstrated that mir-212/132 is expressed on both dorsal and ventral arms of the dentate gyrus in the anterior hippocampus, whereas its expression gets restricted to only dorsal arm of the dentate gyrus in the posterior hippocampus. Scale bars represent $500 \mu \mathrm{m}$.

Figure 9: Relative expression levels of mature miR-212 and miR-132 in thalamus (TH), CA3 and cortex. One mm thick brain sections obtained from the section plane that corresponds to the dorsal hippocampus. Samples from TH, CA3 and cortex were dissected by Harris tissue corer for 
quantitative RT-PCR ${ }^{296}$. The relative fold change was calculated according to TH by setting the $\mathrm{TH}$ values to 1 . A and $\mathrm{B}$ represents two individual mice.

Figure 10: Elevated plus maze, rotarod and explored hole assays on WT and miR-212/132/- mice. (a, b) In the elevated plus maze test, times spent in different zones (a) and the total distance traveled (b) are similar between miR-212/132-/- and WT littermates. (c) In the rotarod test, miR$212 / 132^{-/-}$mice had similar falling latencies in both days compared to the WT controls. (d) In the hole-board test, the number of explored holes by the miR-212/132/- mice is similar to their WT mice. These experiments were conducted by Dr. Konstantin Radyushkin in the research group of Prof. Dr. Dr. Hannelore Ehrenreich.

Figure 11: Sensorimotor gating, sociability, fear conditioning and 8-arm maze assays on WT and miR-212/132-/- mice. (a, b) In the prepulse inhibition test, miR-212/132/- mice demonstrated similar levels of inhibition (a) and startle amplitudes (b) as WT littermates. (c, d) In the tripartite chamber test, both social (c) and memory (d) indices of miR-212/132/- mice were similar to WT controls. (e) In cued and contextual fear conditioning tests, miR-212/132/- mice displayed similar levels of freezing behavior compared to their WT littermates. (f) In 8-arm radial maze task, the number of mistakes done was similar between miR-212/132-/- and WT mice. These experiments were conducted by Dr. Konstantin Radyushkin in the research group of Prof. Dr. Dr. Hannelore Ehrenreich.

Figure 12: Open field and Morris water maze assays on WT and miR-212/132 $2^{-/-}$mice. (a,b) In the open field test, the total distance traveled within $7 \mathrm{~min}$ period was higher for miR-212/132-1compared to WT littermates (a), but the time spent in different zones were similar (b), indicating hyperactivity but no anxiety in miR-212/132 $2^{-/}$mice. (Mann-Whitney U-test, **: p<0.0062, n=2223.) (c-f) In the Morris water maze test, the learning performances of miR-212/132 $2^{-/-}$mice were similar to WT littermates in visible platform version (c), but significantly worse in the hidden platform version of the test (2-way ANOVA for repeated measures for genotype versus trials interaction $\mathrm{F}_{(7,266)}=13.3, \mathrm{p}<0.0001, \mathrm{n}=22-23$;). In the hidden platform version, the time spent in the target quadrant was less for miR-212/132-- mice (Mann-Whitney U-test, *: p<0.003, n=2223) although the average velocity of $\mathrm{miR}-212 / 132^{-/-}$mice was similar to WT mice (f). 
Representative swimming trajectories of WT and miR-212/132/- mice are shown in (e). The place of the hidden platform is shown with a circle within the upper left target quadrant. These experiments were conducted by Dr. Konstantin Radyushkin in the research group of Prof. Dr. Dr. Hannelore Ehrenreich.

Figure 13: (a) Paired-Pulse Ratios (PPRs) are higher in miR-212/132/- mice hippocampal slices compared to WT controls. (Unpaired t-test, two-tailed, *:p<0.05, **:p<0.01, ***:p<0.001) The number of slices (n) analyzed are depicted on the graphs. Representative fEPSP traces for both experiments are shown above the corresponding graphs. (b) Input-output curve of field excitatory postsynaptic potential (fEPSP) at Schaffer collateral-CA1 synapses is similar in both miR$212 / 132^{-/-}$and WT hippocampal slices. These experiments were conducted by Dr. JeongSeop Rhee. .79

Figure 14: Early-phase LTP (E-LTP) and late-phase LTP (L-LTP) on WT and miR-212/132-1hippocampal slices. (a) E-LTP was similar in miR-212/132 $2^{-/-}$and WT hippocampal slices, whereas (b) late-phase LTP was significantly enhanced in miR-212/132/- slices compared to WT slices. The number of slices (n) analyzed are depicted on the graphs. (Unpaired t-test, two tailed, at analyzed time points: $0.001<\mathrm{p}<0.01$ ). These experiments were conducted by Dr. JeongSeop Rhee

Figure 15: LTD on miR-212/132 ${ }^{-/-}$and WT hippocampal slices. LFS application $(1 \mathrm{~Hz}$ for 15 min) showed that NMDAR-dependent LTD levels were not altered in miR-212/132hippocampal slices. The number of slices (n) analyzed are depicted on the graphs. This experiment was conducted by Dr. JeongSeop Rhee.

Figure 16: Depotentiation experiment on miR-212/132-/- and WT hippocampal slices. (a,b) Induction of the depotentiation $40 \mathrm{~min}$ (a) or $2 \mathrm{~min}$ (b) after the HFS showed no difference in depotentiation capacity of miR-212/132/- hippocampal slices compared to WT slices. 2 trains of $100 \mathrm{~Hz}$ for $1 \mathrm{sec}$ were applied as HFS. $2 \mathrm{~Hz}$ for $10 \mathrm{~min}$ or $1 \mathrm{~Hz}$ for $15 \mathrm{~min}$ was applied as LFS. The number of slices (n) analyzed are depicted on the graphs. Representative fEPSP traces for all 
experiments are shown above the corresponding graphs. These experiments were conducted by Dr. JeongSeop Rhee. 82

Figure 17: MecP2, SIRT1 and p250GAP levels in WT and miR-212/132-- mice hippocampus. (a) Approximately 2 months old male mice hippocampi were dissected and the total protein was isolated. Equal concentration of protein was loaded into each lane. Every lane represents an individual mouse sample. (b) The densitometric analysis was performed by Fiji software. $\alpha$ tubulin was used as endogenous control. Unpaired t-test, two-tailed, $\mathrm{p}>0.05,+/+: \mathrm{n}=5 ;-/-: \mathrm{n}=7.84$

Figure 18: Increase in miR-212, -132 and -134 levels. $30 \mathrm{M}$ kainic acid treatment for $6 \mathrm{hrs}$ upregulated mature miR-212 (a), miR-132 (b) and miR-134 (c) production. Paired t-test, twotailed p-value, $* *:<0.01, * * * *:<0.0001, \mathrm{n}=6$.

Figure 19: C-Fos transcript levels in cultured hippocampal neurons. $30 \mathrm{M}$ kainic acid treatment was performed for indicated amount of time. Relative fold changes were calculated by comparing the time points to the untreated group of the same genotype. Bonferroni multiple comparison test, ****: $\mathrm{p}<0.0001$. 2-way ANOVA for genotype versus treatment interaction $\mathrm{F}_{(3,23)}=20.34$, $p<0.0001$; for difference between genotypes $F_{(1,23)}=149.76, p<0.0001$; for difference over treatment time $\mathrm{F}_{(3,23)}=44.77, \mathrm{p}<0.0001$, untreated; $\mathrm{n}=4,30 \mathrm{~min} ; \mathrm{n}=4,3 \mathrm{hrs} ; \mathrm{n}=3,6 \mathrm{hrs} ; \mathrm{n}=4 \ldots . .86$

Figure 20: Arc transcript levels in cultured hippocampal neurons. $30 \mathrm{M}$ kainic acid treatment was performed for indicated amount of time. Relative fold changes were calculated by comparing the time points to the untreated group of the same genotype. Bonferroni multiple comparison test, $* * * *: \mathrm{p}<0.0001, * *: \mathrm{p}<0.01$. 2-way ANOVA for genotype versus treatment interaction $\mathrm{F}_{(3,23)}=$ 17.8, $\mathrm{p}<0.0001$; for difference between genotypes $\mathrm{F}_{(1,23)}=84.31, \mathrm{p}<0.0001$; for difference over treatment time $\mathrm{F}_{(3,23)}=32.86, \mathrm{p}<0.0001$, untreated; $\mathrm{n}=4,30 \mathrm{~min} ; \mathrm{n}=4,3 \mathrm{hrs} ; \mathrm{n}=3,6 \mathrm{hrs} ; \mathrm{n}=4 \ldots . .88$

Figure 21: BDNF transcript levels in cultured hippocampal neurons. $30 \mathrm{M}$ kainic acid treatment was performed for indicated amount of time. Relative fold changes were calculated by comparing the time points to the untreated group of the same genotype. Bonferroni multiple comparison test, $* * * *: \mathrm{p}<0.0001, * *: \mathrm{p}<0.01$. 2-way ANOVA for genotype versus treatment interaction $\mathrm{F}_{(3,23)}=$ 
$6.8, \mathrm{p}=0.0018$; for difference between genotypes $\mathrm{F}_{(1,23)}=30.06, \mathrm{p}<0.0001$; for difference over treatment time $\mathrm{F}_{(3,23)}=31.24, \mathrm{p}<0.0001$, untreated; $\mathrm{n}=4,30 \mathrm{~min} ; \mathrm{n}=4,3 \mathrm{hrs} ; \mathrm{n}=3,6 \mathrm{hrs} ; \mathrm{n}=4 \ldots . .89$

Figure 22: Relative transcript levels at different time points of kainic acid treatment. $30 \mathrm{M}$ of kainic acid treatment was performed for (b) $30 \mathrm{~min}$, (c) $3 \mathrm{hrs}$, (d) 6hrs. (a) No treatment was applied on the control group. The transcript levels of $+/+$ groups were set to 1 and relative transcript levels in -/- groups were calculated. Unpaired t-test, two tailed, *: p<0.05, **: $\mathrm{p}<0.01$, ***:p<0.001, ntreated; $\mathrm{n}=10,30 \mathrm{~min} ; \mathrm{n}=4,3 \mathrm{hrs} ; \mathrm{n}=3,6 \mathrm{hrs} ; \mathrm{n}=9$.

Figure 23: Proposed molecular model. SIRT1 represses miR-134. CREB is downregulated by miR-134 $4^{325}$. The phosphorylation of CREB is driven by neuronal activity ${ }^{313,323}$. Neuronal activity regulated miR-212/132 expression is induced by pCREB (refer to section 1.2.1). MiR-212/132 would be the link between CREB and SIRT1, which constitute a feedback loop. 91

Figure 24: Mature miR-134 levels in cultured hippocampal neurons. (a) Relative miR-134 levels in naïve miR-212/132 $2^{-/}$hippocampal neurons were calculated according to the miR-134 levels in +/+ neurons. (b) $30 \mathrm{M}$ kainic acid treatment was performed for $6 \mathrm{hrs}$. Relative fold changes were calculated by comparing the time point to the untreated group of the same genotype. Unpaired ttest, two-tailed, *: $\mathrm{p}<0.05, * *: \mathrm{p}<0.01, \mathrm{n}=6$. .92

Figure 25: Western blotting for CREB, pCREB and SIRT1. (a) $10 \mathrm{M}$ of forskolin treatment was performed on cultured hippocampal neurons for $30 \mathrm{~min}$. DMSO was applied to untreated group of cells. Equal amount of total protein was loaded into each lane, which represents a replica. Densitometry analysis was performed by Fiji software. (b, d) pCREB level was normalized according to the CREB levels. (c, e) SIRT1 level was normalized according to the $\alpha$-tubulin levels. The pCREB (b) and SIRT1 (c) levels in forskolin untreated groups of both genotypes were set to 1. Relative amounts of pCREB and SIRT1 was calculated according to the untreated group. The pCREB (d) and SIRT1 (e) levels in untreated and forskolin treated groups were compared for different genotypes by setting the protein amount of $+/+$ group to 1 and calculating the protein amount in -/- accordingly. (b) Paired t-test, two-tailed, *: p<0.05, **: p<0.01, (d,e) Bonferroni multiple comparison test, $*: p<0.05$ .94 


\section{Table Legends}

Table 1: Experimentally confirmed targets of miR-132 and miR-212. ....................................14

Table 2: The percentages of projections that EC receives from different associational cortices...34

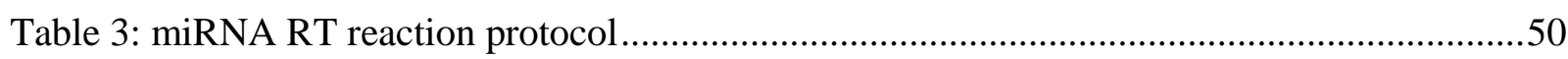

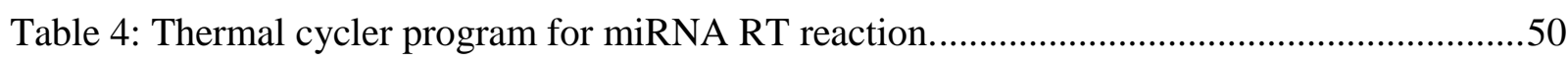

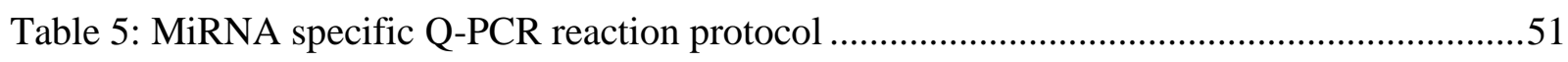

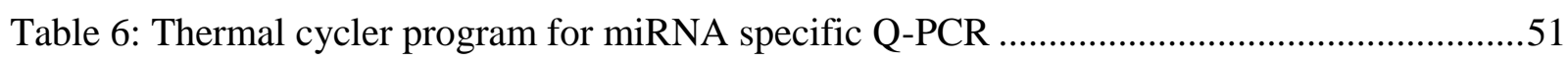

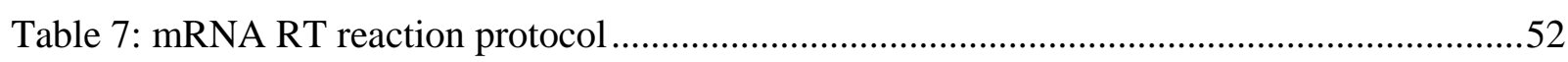

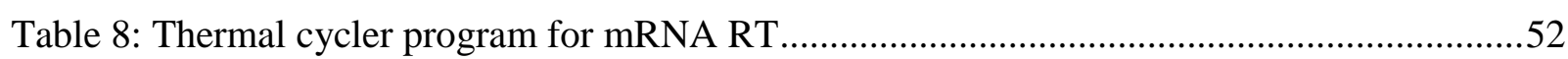

Table 9: Q-PCR reaction formula for gene expression assay …..............................................52

Table 10: Thermal cycler program for gene expression assay..................................................53

Table 11: Primary antibodies used for western blotting experiments........................................58

Table 12: Secondary antibodies used for western blotting experiments. .....................................58

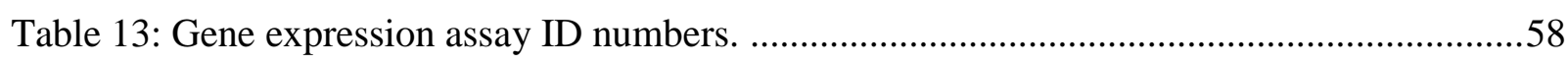

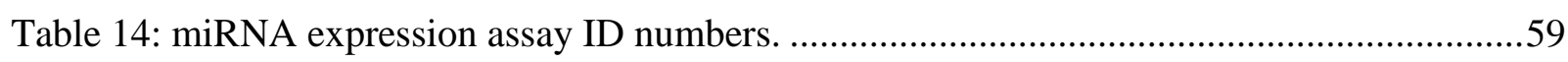




\section{Resume}

Mail: Max Planck Institut für Biophysikalische

Chemie, Am Fassberg

11,Göttingen/Germany

Tel: $\quad$ +495512011567

Email: eerikci@mpibpc.mpg.de

\section{EDUCATION}

January 2010-Present Max Planck Institute for Biophysical Chemistry, Göttingen, GERMANY

$\mathrm{PhD}$, Cell and Molecular Biology

Advisor: Dr. Kamal Chowdhury

Thesis Project: Elucidation of in vivo functions of miR-212/132 in brain development and behavior

2007-2009 Bilkent University, Ankara, TURKEY

M.S., Molecular Biology and Genetics

Advisor: Assoc. Prof. hsan Gürsel

Thesis Project: Enhanced immunomodulatory applications of nucleic acid encapsulating liposomes

Cumulative GPA: 3.96

2003-2007 Bilkent University, Ankara, TURKEY

B.S., Molecular Biology and Genetics

\section{Favorite Courses}

- Molecular Biology of the Cell, Stem Cell Biology, Molecular Biology of the Gene, Immunology, Chemistry.

Cumulative GPA: 3.40

2000-2003ＯDTÜ Gelistirme Vakfı Özel Lisesi, Ankara, TURKEY

High School Diploma 
Ranked second at graduation

$1997-2000$ ODTÜ Gelistirme Vakfı Özel Lisesi, Ankara, TURKEY

Secondary School Diploma

Ranked first at graduation

\section{ATTENDED CONGRESS AND PRESENTATIONS}

- Poster presentation at " $10^{\text {th }}$ International PhD Student Symposium 'Horizons in Molecular Biology', 9-12 September 2013, Göttingen, Germany": MicroRNA-212/132 Gene Family is Involved in Spatial Memory and LTP, 2013, E. Erikçi, A. Uçar, K. Radyushkin, D. Malzahn, H. Ehrenreich, J. S. Rhee, K. Chowdhury.

- Poster presentation at " 2 nd $G G N B$ Science Day - 29 November 2011, Göttingen, Germany": Elucidation of in vivo Functions of miR-212/132 in Brain Development and Behavior, 2011, E. Erikçi, A. Uçar, K. Chowdhury.

- Oral presentation at " $5^{\text {th }}$ National Nanoscience and Nanotechnology Congress - 08-12 June 2009, Eskişehir, Turkey": Enhanced Immunoregulatory Applications of Nucleic Acid Encapsulating Nanoliposomes, 2009, E.Erikçi, K. Karatepe, G. Baysal, B. Biterge, . Gürsel.

- Poster presentation at "World Immune Regulation Meeting - 22-25 Mach 2009, Davos, Switzerland": Encapsulating nucleic acid TLR ligans in nanoliposomes induce differential innate immune activation, 2009, E. Erikçi, . Gürsel.

- "Emerging Topics in Human Functional Genomics and Proteomics" 26-31 Mart 2006, Antalya, Turkey" workshop attendance certificate

\section{SCHOLARSHIPS}

September 2009-Present

Fellowship of Max Planck Institute for Biophysical Chemistry (Doktorandenstipendium)

March 2009

Travel grant from Turkish Society of Immunology for

attending "World Immune Regulation Meeting" held on 22-25 March 2009 in Davos, Switzerland.

September 2007-August 2009Turkish Scientific Research and Development Institution

(TÜB TAK) Scholarship 


\section{PUBLICATIONS:}

- Erikci, E., Gursel, M., and Gursel, I. (2011). Differential immune activation following encapsulation of immunostimulatory $\mathrm{CpG}$ oligodeoxynucleotide in nanoliposomes. Biomaterials 32, 1715-1723.

- Ucar, A., Gupta, S.K., Fiedler, J., Erikci, E., Kardasinski, M., Batkai, S., Dangwal, S., Kumarswamy, R., Bang, C., Holzmann, A., et al. (2012). The miRNA-212/132 family regulates both cardiac hypertrophy and cardiomyocyte autophagy. Nat Commun 3, 1078.

- Chevrier, S., Emslie D., Shi W., Kratina T., Wellard C., Karnowski A., Erikci E., Smyth G. K., Chowdhury K., Tarlinton D., Corcoran L. M. (2014). The BTB-ZF transcription factor Zbtb20 is driven by Irf4 to promote plasma cell differentiation and longevity. Journal of Exp. Med. (under review) 